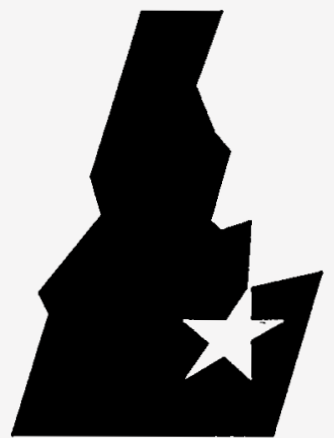

Idaho

National

Engineering

Laboratory
T.in

$$
\begin{aligned}
& \left\{\begin{array}{c}
a ? 2 ? j \\
\ldots
\end{array}\right. \\
& \text { (a) }
\end{aligned}
$$

May, 1996

\title{
Fully Implicit Kinetic Modelling of Collisional Plasmas
}

\section{A. Mousseau}

\section{DISCLAIMER}

This report was prepared as an account of work sponsored by an agency of the United States Government. Neither the United States Government nor any agency thereof, nor any of their employees, makes any warranty, express or implied, or assumes any legal liability or responsibility for the accuracy, completeness, or usefulness of any information, apparatus, product, or process disclosed, or represents that its use would not infringe privately owned rights. Reference herein to any specific commercial product, process, or service by trade name, trademark, manufacturer, or otherwise does not necessarily constitute or imply its endorsement, recommendation, or favoring by the United States Government or any agency thereof. The views and opinions of authors expressed herein do not necessarily state or reflect those of the United States Government or any agency thereof.

$$
\mid
$$





$$
\text { INEL- } 96 / 0149
$$

INEL-96/0149

Fully Implicit Kinetic Modelling of Collisional Plasmas

Vincent A. Mousseau

Published May 1996

Idaho National Engineering Laboratory Lockheed Martin Idaho Technologies

Idaho Falls, Idaho 83415

Supported by the

U.S. Department of Energy

through DOE Idaho Operations Office

Contract DE-AC07-94ID13223 


\section{AUTHORIZATION TO SUBMIT}

\section{DISSERTATION}

This dissertation of Vincent A. Mousseau, submitted for the degree of Doctor of Philosophy and titled "Fully Implicit Kinetic Modelling of Collisional Plasmas," has been reviewed in final form, as indicated by the signatures and dates given below. Permission is now granted to submit final copies to the College of Graduate Studies for approval.

Major Professor

Committee Members

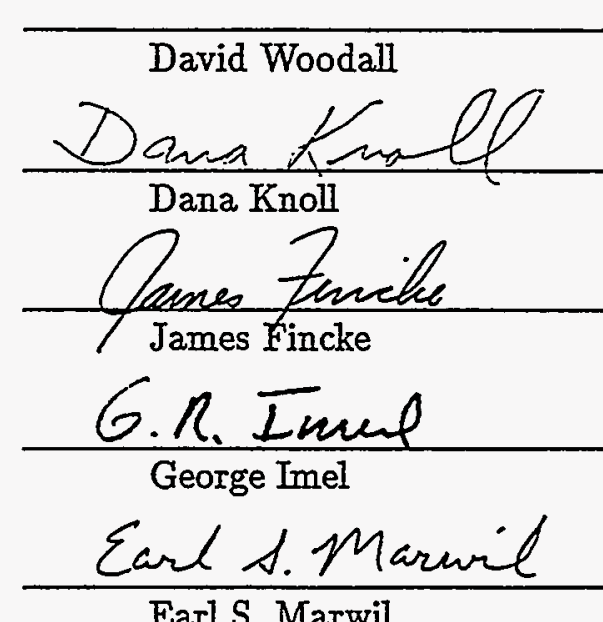

Earl S. Marwil
Date

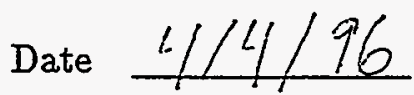

Date $4 / 4 / 96$

Date $4 / 4196$

Date $4 / 4 / 96$

Date

Steve Penoncello

Department

Administrator

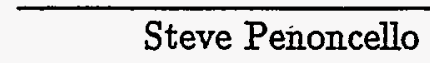

Engineering

College Dean
Date

Richard T Jacobsen

Final Approval and Acceptance by the College of Graduate Studies

Jean'ne M. Shreeve

Date 



\section{Fully Implicit Kinetic Modelling of Collisional Plasmas}

Vincent Andrew Mousseau, Ph.D.

University of Idaho, 1996

Adviser: David Woodall, Ph.D.

This dissertation describes a numerical technique, Matrix-Free Newton Krylov, for solving a simplified Vlasov-Fokker-Planck equation. This method is both deterministic and fully implicit, and may not have been a viable option before current developments in numerical methods. Results are presented that indicate the efficiency of the Matrix-Free Newton Krylov method for these fully-coupled, non-linear integro-differential equations. The use and requirement for advanced differencing is also shown. To this end, implementations of Chang-Cooper differencing and flux limited Quadratic Upstream Interpolation for Convective Kinematics (QUICK) are presented. Results are given for a fully kinetic ion-electron problem with a self consistent electric field calculated from the ion and electron distribution functions. This numerical method, including advanced differencing, provides accurate solutions, which quickly converge on workstation class machines.

It is demonstrated that efficient steady-state solutions can be achieved to the non-linear integro-differential equation, obtaining quadratic convergence, without incurring the large memory requirements of an integral operator. Model problems are presented which simulate plasma impinging on a plate with both high and low neutral particle recycling typical of a divertor in a Tokamak device. These model problems demonstrate the performance of the 
new solution method. 


\section{Acknowledgments}

I wish to thank the many people that were involved in the development of this work. Dr. David Woodall and Dr. Dana Knoll who acted as my advisors throughout this research. I am grateful to the members of my committee, Dr. Fincke, Dr. Imel, and Dr. Marwil for their review of this work, and related suggestions. I wish to thank my colleagues who helped me with insightful discussions; Drs. Montierth, Chang, McHugh, Ramshaw, Johnson, Mortensen, Douglass, Nguyen, and soon to be Dr. Hansen and other members of the INEL staff. I also would like to thank my wife Kimberlyn for her support throughout this work. This work was performed under the auspices of the U.S. Dept. of Energy (DOE), Idaho Operations Office, under DOE Contract No. DE-AC07-94ID13223, and supported by the Idaho National Engineering Laboratory through the Long Term Research Initiative in Computational Mechanics and the Laboratory Directed Research and Development programs. 


\section{Contents}

Authorization Form

Abstract

Acknowledgements

Table of Contents

List of Figures

List of Tables

$x v$

List of Symbols

xvii

1 Introduction

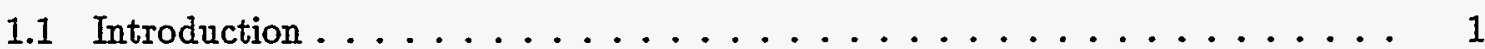

1.2 Fokker-Planck ....................... 4

1.3 Why Kinetic Modeling? . . . . . . . . . . . . . . . . 8

1.4 The Tokamak Edge Plasma . . . . . . . . . . . . . . . . 12

1.5 General Numerical Approach ..................... 16

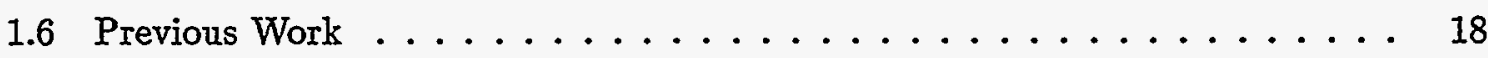




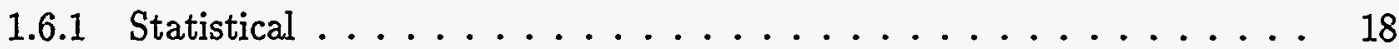

1.6 .2 Deterministic . . . . . . . . . . . . . . . 20

1.7 Overview of Dissertation . . . . . . . . . . . . . . 22

2 One Spatial One Velocity (1D1V) Ion Only 25

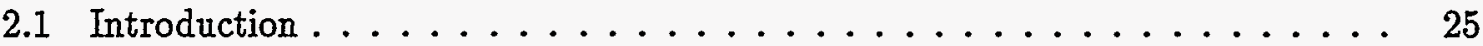

2.2 Mathematical Model . . . . . . . . . . . . . . . . . 26

2.2.1 Fokker-Planck Collision Operator . . . . . . . . . . . . 28

2.2 .2 Neutral Ionization Model . . . . . . . . . . . . . . . 28

2.2 .3 Core Source . . . . . . . . . . . . . . . . . . 31

2.2 .4 Electric Field . . . . . . . . . . . . . . . . 32

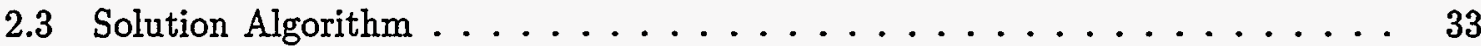

2.3 .1 Fully Implicit Motivation . . . . . . . . . . . . . . 33

2.3.2 Newton's Method . . . . . . . . . . . . . . . . 35

2.3 .3 Krylov Method . . . . . . . . . . . . . . . 38

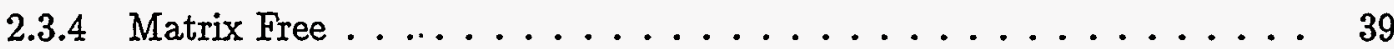

2.4 Model Problem and Results . . . . . . . . . . . . . . . . 40

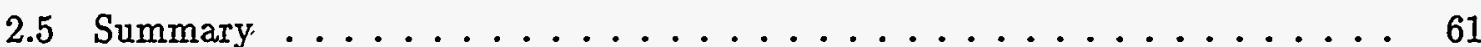

3 One Velocity (OD1V) Ion and Electron $\quad 62$

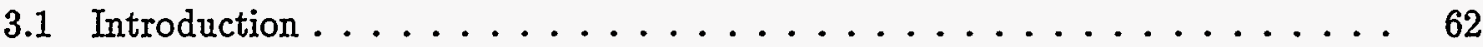

3.2 Mathematical Model ...................... 63

3.3 New Matrix Structure ...................... 65

3.4 Differencing the Fokker-Planck Collision Operator . . . . . . . . . . 66

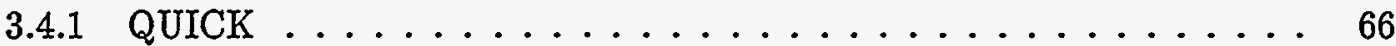


viii

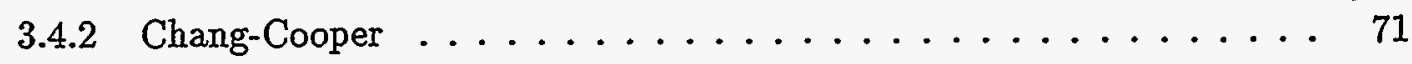

3.5 Model Problem and Results . . . . . . . . . . . . . . 75

3.5.1 Exact Solution for Resistivity Calculation ............ 76

3.5.2 Results for Resistivity Calculation . . . . . . . . . . 77

3.5.3 Exact Solution Null Problem (Maxwellian) . . . . . . . . . 77

3.5.4 Results for the Null Problem (Maxwellian) . . . . . . . . . . 81

3.5.5 Exact Solution for Thermal Equilibrium . . . . . . . . . . . . 89

3.5.6 Results for Thermal Equilibrium Calculation . . . . . . . . . 96

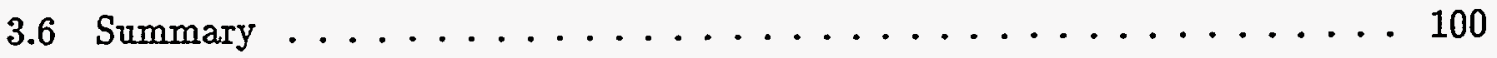

4 Higher Order Differencing $\quad 102$

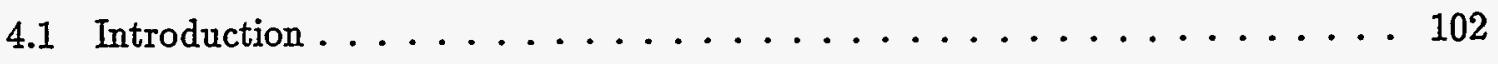

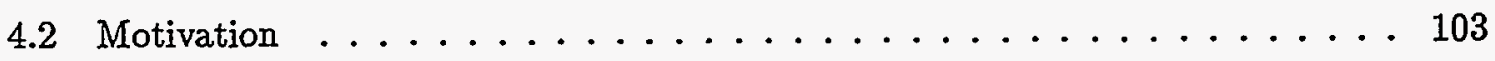

4.3 Numerical Schemes . . . . . . . . . . . . . . . 106

4.3.1 Interpolated Donor Cell (IDC) Differencing . . . . . . . . . . 107

4.3.2 Non-Linear Differencing (Flux Limiting) . . . . . . . . . . 108

4.4 Mathematical Model . . . . . . . . . . . . . . . . . . 121

4.4 .1 Exact Solution ........................... 122

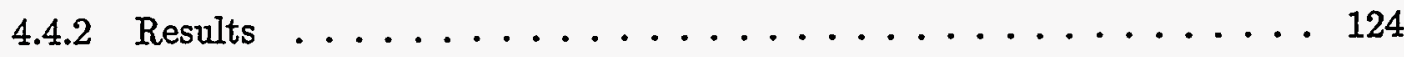

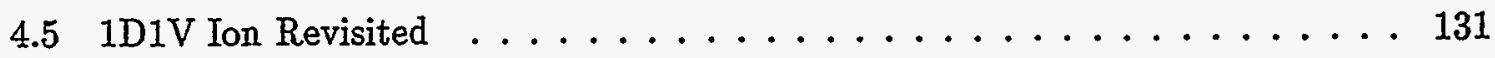

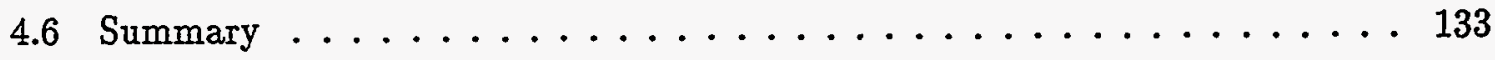

5. One Velocity One Spatial Ion and Electron 135

5.1 Introduction . . . . . . . . . . . . . . . 135

5.2 Mathematical Model . . . . . . . . . . . . . . . . 136 
5.3 Quasi-Neutral Electric Field . . . . . . . . . . . . . . 136

5.4 Test 1: Plasma Impinging on a Surface . . . . . . . . . . . 139

5.5 Test 2: High Recycling . . . . . . . . . . . . . . . 144

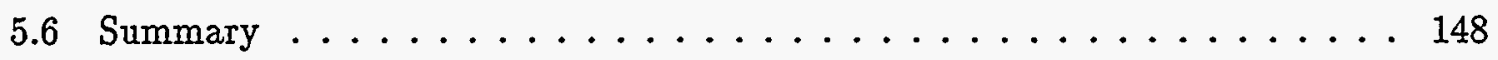

6 Summary 149

6.1 Summary and Conclusions . . . . . . . . . . . . . . . . 149

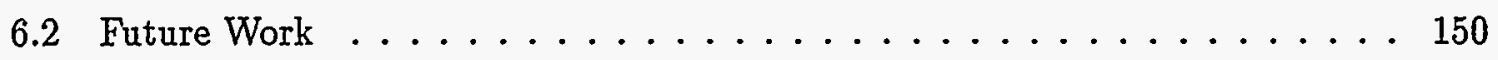

A Taylor Series for Central and Upwind Differencing 152

A.1 Forward, Backward, and Upwind . . . . . . . . . . . . 152

A.2 Central Differencing . . . . . . . . . . . . . . . . 154

B Differentiable Flux Limiter Derivation $\quad 155$

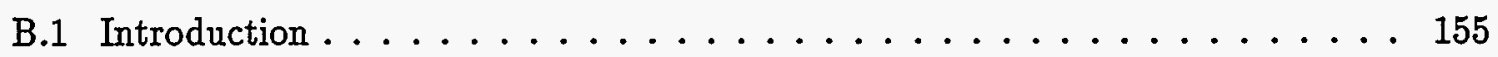

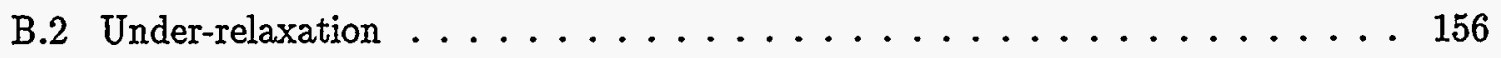

B.3 Non-Dimensional Non-Differentiable . . . . . . . . . . . . 158

B.4 Non-Dimensional Differentiable . . . . . . . . . . . . 160

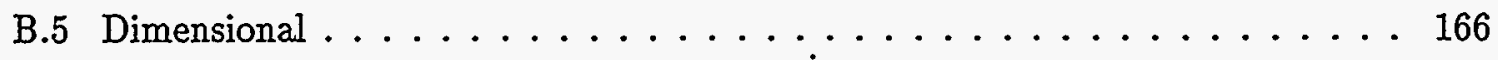

$\begin{array}{ll}\text { Bibliography } & 171\end{array}$ 


\section{List of Figures}

1.1 Tokamak Geometry . . . . . . . . . . . . . . . . . 13

1.2 Tokamak Cross Section ........................ 13

2.1 Model Problem Geometry . . . . . . . . . . . . . . . . . 41

2.2 Neutral Number Density . . . . . . . . . . . . . . . . . 42

2.3 Electric Field . . . . . . . . . . . . . . . . . . . 42

2.4 Ion Number Density ......................... 43

2.5 Fluid Velocity . . . . . . . . . . . . . . . . . 43

2.6 Fluid Pressure $\ldots \ldots \ldots \ldots \ldots \ldots \ldots \ldots \ldots \ldots$

2.7 Fluid Temperature . . . . . . . . . . . . . . . . . 44

2.8 Distribution Function Slices . . . . . . . . . . . . . 45

2.9 Mesh Sequenced Convergence vs. Newton Iteration . . . . . . . . . . . 46

$2.10200 \times 400$ Convergence vs. CPU Time . . . . . . . . . . . . 47

2.11 Mesh Sequenced Pressure . . . . . . . . . . . . . . 48

2.12 Mesh Sequenced Ion Density . . . . . . . . . . . . . 48

2.13 Matrix Free vs. Lagged Integral CPU Time . . . . . . . . . . . . . 49

2.14 Matrix Free vs. Lagged Integral Newton Iterations . . . . . . . . . . 50 
2.15 Fluid Pressure vs. Neutral MFP $\ldots \ldots \ldots \ldots \ldots$

2.16 Ion Number Density vs. Neutral MFP . . . . . . . . . . . . . 51

2.17 Matrix Free vs. Lagged Integral CPU Time for Variable Collision Frequencies 52

2.18 Matrix Free vs. Lagged Integral Newton Iteration for Variable Collision Fre-

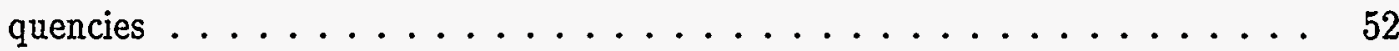

2.19 Collision Frequency vs. Distance $\ldots \ldots \ldots \ldots \ldots \ldots \ldots$

2.20 Ion Number Density Constant vs. Variable Collision Frequency . . . . . . . 53

2.21 Fluid Temperature Constant vs. Variable Collision Frequency . . . . . . . . 54

2.22 Particle Flux vs. Injection Shape . . . . . . . . . . . . . 55

2.23 Distribution Function vs. Injection Shape ............. 55

2.24 Fluid Temperature vs. Injection Shape . . . . . . . . . . . . . 56

2.25 Particle Density vs. Injection Shape . . . . . . . . . . . . . 56

2.26 Average Krylov Iteration vs. Level of ILU Fill-In . . . . . . . . . . 58

2.27 Run Time vs. Level of ILU Fill in for Different Grids . . . . . . . . . . . 58

2.28 Average Krylov Iteration and Run Time vs. Time Steps Size . . . . . . . 59

2.29 Total Krylov Iteration vs. Krylov Tolerance for Different Maximum Krylov Space Dimensions . . . . . . . . . . . . . . . . . 60

3.1 Matrix Coupling for Ions and Electrons with Unlike Particle Collisions . . . . 65

3.2 QUICK Differencing . . . . . . . . . . . . . . 67

3.3 Different Non-Uniform Meshes . . . . . . . . . . . . . . 69

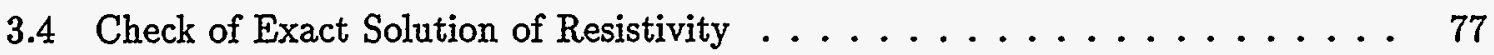

3.5 Velocity Transients for Resistivity Calculations . . . . . . . . . . . 78

3.6 Upwind Differencing 200 Cells Initial Collision Operator Values . . . . . . . 82 
3.7 Upwind Differencing 200 Cells Initial Normalized Error . . . . . . . . . 83

3.8 Upwind Differencing 200 Cells Final Collision Operator Values . . . . . . 84

3.9 Upwind Differencing 200 Cells Final Normalized Error . . . . . . . . . . . 85

3.10 Upwind Differencing 200 Cells Temperature Transient . . . . . . . . . 86

3.11 Quick Differencing 200 Cells Initial Collision Operator Values . . . . . . . 86

3.12 Quick Differencing 200 Cells Initial Normalized Error . . . . . . . . . . . 87

3.13 Quick Differencing 200 Cells Temperature Transient . . . . . . . . . 88

3.14 Chang-Cooper IDC Differencing 50 Cells Initial Collision Operator Values . . 89

3.15 Chang-Cooper IDC Differencing 50 Cells Initial Normalized Error . . . . . . 90

3.16 Chang-Cooper IDC Differencing 50 Cells Temperature Transient . . . . . . 90

3.17 Upwind Equilibrium Calculations . . . . . . . . . . . . 97

3.181600 Cell Upwind Temperature Transient $\ldots \ldots \ldots \ldots \ldots$

3.19 Upwind Differencing 200 cells . . . . . . . . . . . . . . . . 99

3.20 Quick Differencing 200 cells . . . . . . . . . . . . . . . 99

3.21 Chang-Cooper Interpolated Donor Cell Differencing 50 cells . . . . . . . 100

4.1 Upwind Discretization Mesh . . . . . . . . . . . . . . . . 103

4.2 Flux Limiting Example . . . . . . . . . . . . . . . . . . . . 109

4.3 Non-Dimensional Flux Limiting Criteria . . . . . . . . . . . . . 111

4.4 Flux Limiting Example $2 \ldots \ldots \ldots$. . . . . . . . . . . . . . 113

4.5 Degenerate Case of a Diagonal Discontinuity . . . . . . . . . . . 115

4.6 Components of the Flux Limiter . . . . . . . . . . . . . 118

4.7 Non-Differentiable (Rough) Flux Limiter . . . . . . . . . . . . . . . . 119

4.8 Differentiable (Smooth) Flux Limiter . . . . . . . . . . . . . . . 120 
4.9 Differentiable vs. Non-Differentiable Limiter Convergence for a Steady State

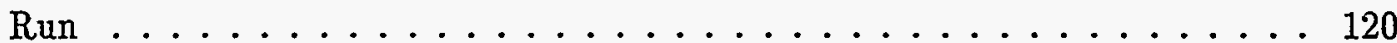

4.10 Differentiable vs. Non-Differentiable Limiter Convergence for a Transient Run 121

4.11 Upwind Differencing Temperature Single Maxwellian . . . . . . . . . . . 125

4.12 Upwind Differencing Distribution Function Single Maxwellian . . . . . . . 126

4.13 Flux Limited QUICK Differencing Temperature Single Maxwellian . . . . . . 127

4.14 Flux Limited QUICK Differencing Distribution Function Single Maxwellian . 127

4.15 Distribution Function for the "Square Wave" . . . . . . . . . . . . 128

4.16 Temperature for the Double Maxwellian . . . . . . . . . . . . . . . . . 129

4.17 Distribution Function for the Double Maxwellian . . . . . . . . . . . 130

4.18 Various Discretization Schemes on a $25 \times 50$ Grid . . . . . . . . . . . 131

4.19 Various Discretization Schemes on a $100 \times 200$ Grid $\ldots \ldots \ldots \ldots$

4.20 Mesh Convergence for QUICK-Chang-Cooper . . . . . . . . . . . 133

4.21 QUICK vs. Change-Cooper Collision Differencing . . . . . . . . . . . . 134

5.1 Number Densities on the $25 x 50$ Grid . . . . . . . . . . . . . . 140

5.2 Electric Field on the $25 \times 50$ Grid $\ldots \ldots \ldots \ldots \ldots$

5.3 Particle Flux on the $100 \times 200$ Grid . . . . . . . . . . . . . 141

5.4 Fluid Velocity on the $25 \times 50$ Grid . . . . . . . . . . . . . . 142

5.5 Mach Number on the $25 \times 50$ Grid . . . . . . . . . . . . . . . . . 142

5.6 Newton Convergence on the $25 \times 50$ Grid . . . . . . . . . . . . 143

5.7 Mesh Sequenced Mach Numbers . . . . . . . . . . . . . . . . . 144

5.8 Mesh Sequenced Newton Convergence for the Ion Equation . . . . . . . 145

5.9 Ion and Electron Temperatures for High and Low Recycling Rate for Neutrals 146 
5.10 Half Distribution Function for High and Low Recycling . . . . . . . . 147

5.11 High Recycling Number Densities on the $50 \times 100$ Grid . . . . . . . . 147

5.12 High Recycling Collision Frequencies . . . . . . . . . . . . . 148

A.1 Upwind Discretization Mesh . . . . . . . . . . . . . 152

B.1 Differentiable (Smooth) and Non-Differentiable (Rough) Limiters . . . . . . 156

B.2 Differentiable vs. Non-Differentiable Limiter Convergence for a Steady State

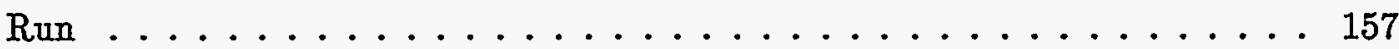

B.3 Differentiable and Non-Differentiable Limiters point $1 \ldots \ldots$. . . . . 161

B.4 Differentiable and Non-Differentiable Limiters point $2 \ldots \ldots$. . . . . 162

B.5 Differentiable and Non-Differentiable Limiters point $3 \ldots \ldots$. . . . . 164

B.6 Differentiable and Non-Differentiable Limiters point $4 \ldots \ldots \ldots$ 


\section{List of Tables}

2.1 Input Values $\ldots \ldots \ldots \ldots \ldots \ldots \ldots \ldots \ldots \ldots \ldots \ldots$

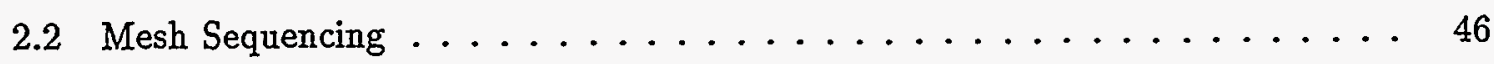

3.1 Null Problem Input Values $\ldots \ldots \ldots \ldots \ldots \ldots \ldots \ldots \ldots$

3.2 Typical Numerical Maxwellian Input Values . . . . . . . . . . 80

3.3 Thermal Equilibrium Problem Input Values $\ldots \ldots \ldots \ldots \ldots$

4.1 Flux Limit Example Input Values . . . . . . . . . . . . . . . . 109

4.2 Flux Limiting Special Cases . . . . . . . . . . . . . 112

4.3 Method of Characteristics Solution . . . . . . . . . . 123

4.4 Single Maxwellian Problem Input Values . . . . . . . . . . . . 125

4.5 Relative Error for the Single Maxwellian . . . . . . . . . . . 128

4.6 Double Maxwellian Problem Input Values . . . . . . . . . . . . . 129

4.7 Relative Error for the Double Maxwellian . . . . . . . . . . . 130

5.1 Input Values for Ion-Electron Plasma Impinging on a Wall . . . . . . . 139

5.2 High Recycling Simulation . . . . . . . . . . . . . . 145

B.1 Cubic Spline Data Non-Dimensional $\ldots \ldots \ldots \ldots \ldots \ldots$ 
B.2 Cubic Spline Data Dimensional . . . . . . . . . . . . 167 


\section{List of Symbols}

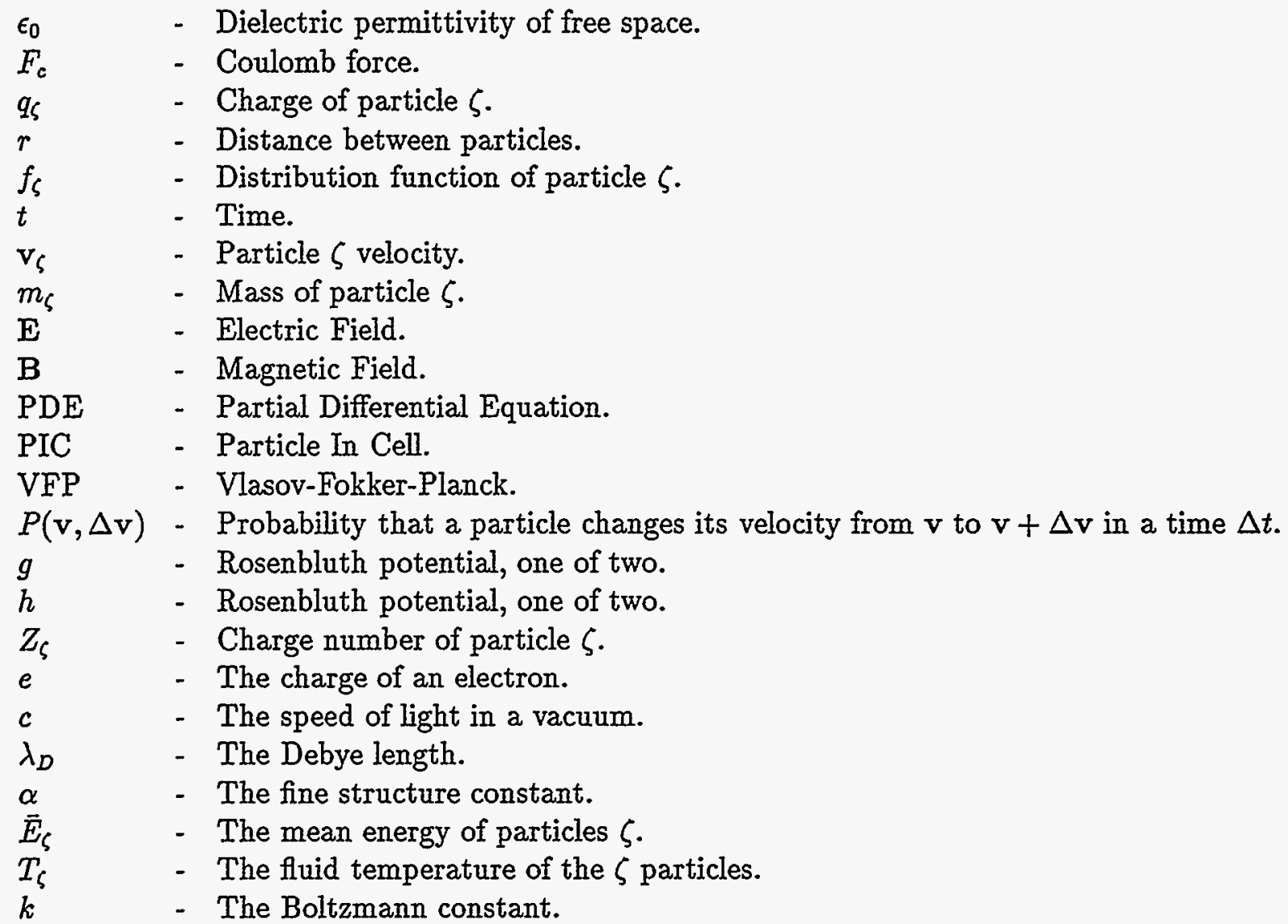




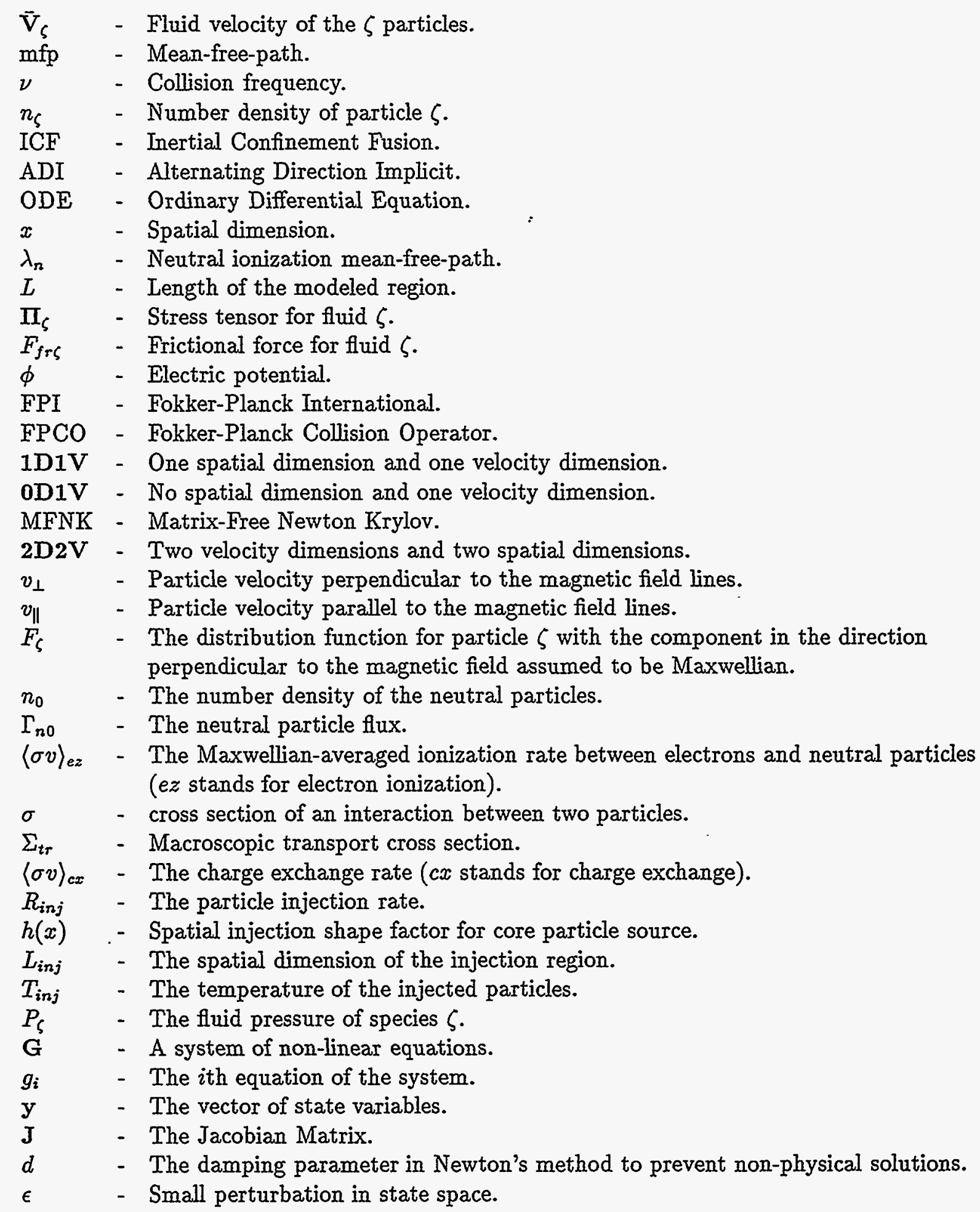




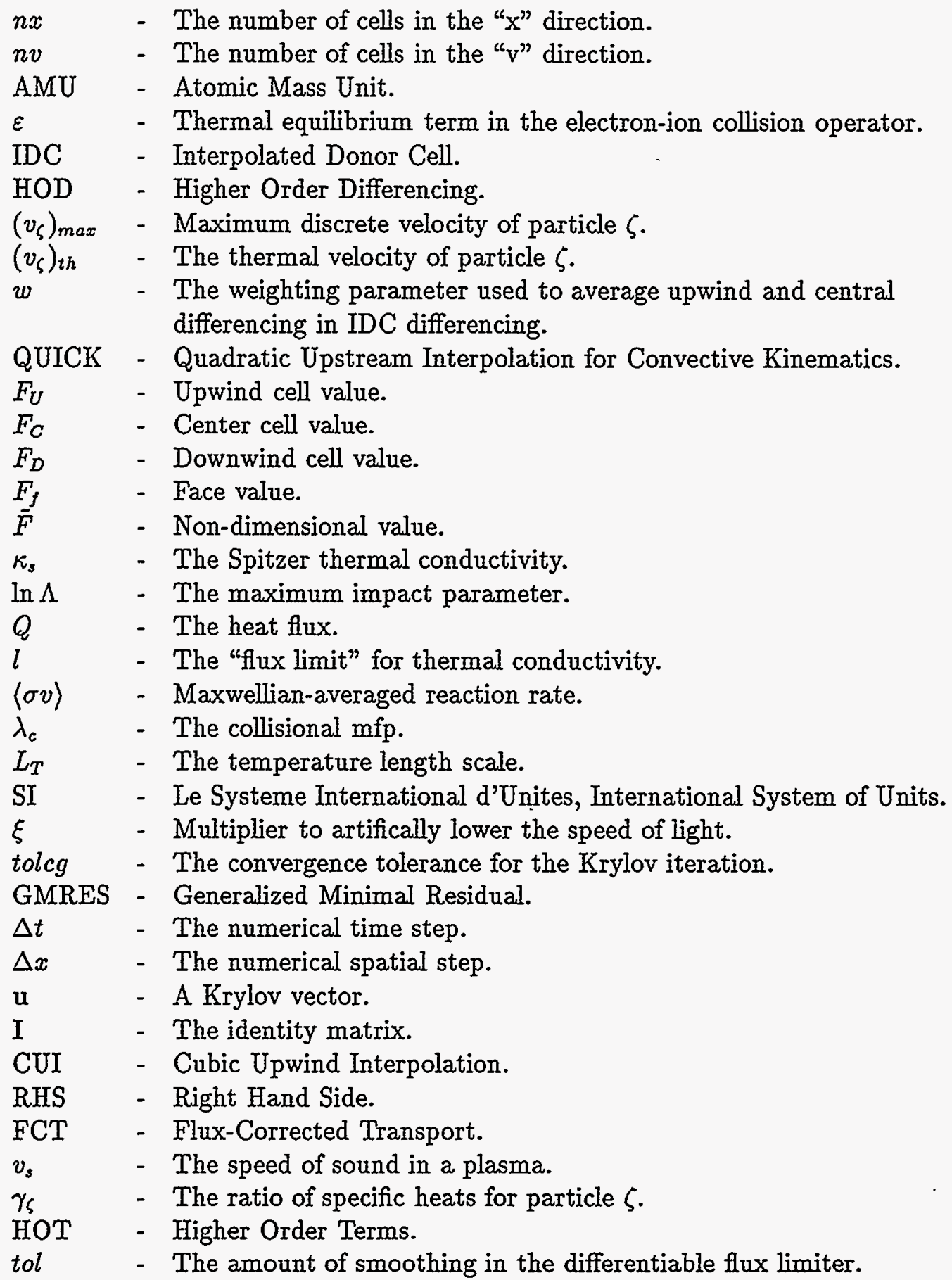





\section{Chapter 1}

\section{Introduction}

\subsection{Introduction}

Computer simulation of plasmas is a difficult problem that has been worked on for many years [1]. The difficulty is illuminated by the definition of a plasma. A plasma is a quasineutral gas of charged and neutral particles which exhibits collective behavior. Quasineutral means that there is approximately the same number of positively charged and negatively charged particles in any given region. This quasineutrality is caused by the interaction between the charged particles and the electric and magnetic fields. Because many charged particles effect the electric and magnetic fields which in turn effect the charged particles, a collective behavior of the plasma results. These effects are what make plasma modeling much more difficult than neutron transport and traditional fluid dynamics which only have short range effects caused by hard body or Boltzmann collisions.

The collisions between charged particles in a plasma are not Boltzmann collisions but are Coulomb collisions instead. Whenever there are two charged particles, there is a Coulomb 
force between them given by,

$$
F_{c}=\frac{1}{4 \pi \epsilon_{0}} \frac{q_{1} q_{2}}{r^{2}}
$$

where $\epsilon_{0}$ is the permittivity of free space, $q_{\zeta}$ is the charge on particle $\zeta$, and $r$ is the distance between particles. From equation 1.1 it is clear that these interactions between charged particles scale as $r^{-2}$ and are therefore refered to as long range interactions.

Models of plasmas can be categorized by the rate of Coulomb collisions. If the rate of Coulomb collisions is high (i.e., the mean free path between collisions is very small) one can use a fluid description to model the plasma [2]. In this approach equations for conservation of mass, momentum and energy are solved simular to classical fluid dynamics. These equations are derived by making assumptions about the particle motions and then determining an average behavior for a large number of particles.

If the collision rate is very small, then the Vlasov equation can be used to model a plasma. The Vlasov equation is,

$$
\frac{\partial f}{\partial t}+\mathbf{v} \cdot \nabla f+\frac{q}{m}(\mathbf{E}+\mathbf{v} \times \mathbf{B}) \cdot \frac{\partial f}{\partial \mathbf{v}}=0
$$

where $f$ is the distribution function (i.e., the density of particles in position velocity phase space) $t$ is time, $v$ is the velocity of the particle, $m$ is the particle mass, $\mathbf{E}$ is the electric field, and $\mathbf{B}$ is the magnetic field. The integral of $f$ over a finite space and velocity volume corresponds to the number of particles at that location with the given velocity. The first term represents the time dependence of the distribution function. The second term accounts for the convective flux. The third term represents the acceleration of the charged particles due to the electric and magnetic fields. 
Solution of the Vlasov equation is difficult because it requires the partial differential equation (PDE) to be solved in seven dimensional space, 1 time, 3 space, and 3 velocities. Because discretizing and solving this equation in state space is difficult, a different approach called the particle in cell (PIC) method [3] was developed.

The PIC method uses a statistical approach to solve the Vlasov equation. First particles which represent many real charged particles are placed randomly in state space (i.e., velocity and space) according to a distribution function. Then the following cycle of four steps is repeated until a statistically smooth solution is achieved.

1. From the particle's position and velocity, compute their contribution to the density and current for the cell that they are in.

2. From the density and current in the cells, compute the electric field and magnetic field for that spatial location.

3. From the magnetic and electric fields, compute the force on the particles for that spatial location.

4. From the force, compute a new particle velocity and spatial location.

The problems of interest for this dissertation are in the intermediate range of Coulomb collision frequency. For these types of problems the collisions have to be represented so a new term is added to the Vlasov equation. It now becomes the Vlasov-Fokker-Planck (VFP) equation,

$$
\frac{\partial f}{\partial t}+\mathbf{v} \cdot \nabla f+\frac{q}{m}(\mathbf{E}+\mathbf{v} \times \mathbf{B}) \cdot \frac{\partial f}{\partial \mathrm{v}}=\left(\frac{\partial f}{\partial t}\right)_{c}
$$

This term, $\left(\frac{\partial f}{\partial t}\right)_{c}$ is the Fokker-Planck collision operator. The Fokker-Planck collision operator represents the Coulomb collisions between the charged particles. Equation 1.3 is the 
equation solved computationally in this dissertation.

\subsection{Fokker-Planck}

The Fokker-Planck equation has the following form,

$$
\left(\frac{\partial f}{\partial t}\right)_{c}=\frac{\partial}{\partial \mathrm{v}} \cdot\left\langle\frac{\Delta \mathrm{v}}{\Delta t}\right\rangle f(\mathrm{v}, t)+\frac{1}{2} \sum_{i, k} \frac{\partial^{2}}{\partial v_{i} \partial v_{k}}\left[\left\langle\frac{\Delta v_{i} \Delta v_{k}}{\Delta t}\right\rangle f(\mathrm{v}, t)\right]
$$

Where,

$$
\left\langle\frac{\Delta \mathrm{v}}{\Delta t}\right\rangle=\frac{1}{\Delta t} \int f(\mathrm{v}, t) P(\mathrm{v}, \Delta \mathrm{v}) \Delta \mathrm{v} d(\Delta \mathrm{v})
$$

and,

$$
\left\langle\frac{\Delta v_{i} \Delta v_{k}}{\Delta t}\right\rangle=\frac{1}{\Delta t} \int f(\mathrm{v}, t) P(\mathrm{v}, \Delta \mathrm{v}) \Delta v_{i} \Delta v_{k} d(\Delta \mathrm{v})
$$

Here $P(\mathbf{v}, \Delta \mathbf{v})$ is the probability that a particle changes its velocity from $\mathbf{v}$ to $\mathbf{v}+\Delta \mathbf{v}$ in a time $\Delta t$ or,

$$
f(\mathrm{v}, t)=\int f(\mathrm{v}-\Delta \mathrm{v}, t-\Delta t) P(\mathrm{v}-\Delta \mathrm{v}, \Delta \mathrm{v}) d(\Delta \mathrm{v})
$$

assuming that the distribution $f(\mathrm{v}, t)$ is only changed by collisions and the summation in equation 1.4 is a double sum over the three coordinate directions. Note also that the probability $P(\mathbf{v}, \Delta \mathrm{v})$ is normalized such that,

$$
\int P(\mathrm{v}, \Delta \mathrm{v}) d(\Delta \mathrm{v})=1
$$

The derivation of the Fokker-Planck equation results from the fact that large angle deflection of a charged particle is typically the result of many small angle collisions rather than 
one large binary collision, as for neutral particle collisions. Since the velocity change is due to small angle collisions, the velocity change, $\Delta v$, in a time, $\Delta t$, is small. Therfore, one can expand $\Delta \mathrm{v}$ in equation 1.7 to yield,

$$
\begin{aligned}
f(\mathrm{v}, t) & =\int\left\{f(\mathrm{v}, t) P(\mathrm{v}, \Delta \mathrm{v})-\Delta t P(\mathrm{v}, \Delta \mathrm{v}) \frac{\partial f}{\partial t}-\Delta \mathrm{v} \frac{\partial}{\partial \mathrm{v}}[f(\mathrm{v}, t) P(\mathrm{v}, \Delta \mathrm{v})]\right. \\
& \left.+\frac{1}{2} \sum_{i} \sum_{k} \Delta v_{i} \Delta v_{k} \frac{\partial^{2}}{\partial v_{i} \partial v_{k}}[f(\mathrm{v}, t) P(\mathrm{v}, \Delta \mathrm{v})]+\cdots\right\} d(\Delta \mathrm{v})
\end{aligned}
$$

where third order and higher terms have been neglected for small $\Delta v$. This can be rewritten to give,

$$
\left(\frac{\partial f}{\partial t}\right)_{c}=\left(\frac{1}{\Delta t} \int-\Delta \mathrm{v} \cdot \frac{\partial}{\partial \mathrm{v}} f P+\frac{1}{2} \sum_{i, k} \Delta v_{i} \Delta v_{k} \frac{\partial^{2}}{\partial v_{i} \partial v_{k}} f P\right)
$$

Using equation 1.5 and equation 1.6 one can see that this becomes equation 1.4 .

$$
\left(\frac{\partial f}{\partial t}\right)_{c}=\frac{\partial}{\partial \mathrm{v}} \cdot\left\langle\frac{\Delta \mathrm{v}}{\Delta t}\right\rangle f(\mathrm{v}, t)+\frac{1}{2} \sum_{i, k} \frac{\partial^{2}}{\partial v_{i} \partial v_{k}}\left[\left\langle\frac{\Delta v_{i} \Delta v_{k}}{\Delta t}\right\rangle f(\mathrm{v}, t)\right] .
$$

This equation has two important physical properties. The first term in this equation contains one of the Fokker-Planck coefficients, $\left\langle\frac{\Delta v}{\Delta t}\right\rangle$, or the coefficient of dynamic friction. This term represents the frictional force between particles. The second Fokker-Planck coefficient, $\left\langle\frac{\Delta v_{i} \Delta v_{k}}{\Delta t}\right\rangle$, or the diffusion tensor, is included in the second term which represents the diffusion of particles in velocity space. For a full Fokker-Planck simulation these two coefficients would be expressed in terms of Rosenbluth potentials [4], $h$ and $g$, instead of the $P$ probability. For 
this method the Fokker-Planck equation is written [5],

$$
\frac{1}{\Gamma_{a}}\left(\frac{\partial f_{a}}{\partial t}\right)_{c}=-\frac{\partial}{\partial v_{i}}\left(f_{a} \frac{\partial h_{a}}{\partial v_{i}}\right)+\frac{1}{2} \frac{\partial^{2}}{\partial v_{i} \partial v_{k}}\left(f_{a} \frac{\partial^{2} g_{a}}{\partial v_{i} \partial v_{k}}\right)
$$

where,

$$
\begin{gathered}
g_{a}=\sum_{b}\left(\frac{Z_{b}}{Z_{a}}\right)^{2} \ln \Lambda_{a b} \int f_{b}\left(\mathrm{v}^{\prime}\right)\left|\mathrm{v}-\mathrm{v}^{\prime}\right| d\left(\mathrm{v}^{\prime}\right), \\
h_{a}=\sum_{b} \frac{m_{a}+m_{b}}{m_{b}}\left(\frac{Z_{b}}{Z_{a}}\right)^{2} \ln \Lambda_{a b} \int f_{b}\left(\mathrm{v}^{\prime}\right)\left|\mathrm{v}-\mathrm{v}^{\prime}\right|^{-1} d\left(\mathrm{v}^{\prime}\right), \\
\Gamma_{a}=\frac{4 \pi Z_{a}^{4} e^{4}}{m_{a}^{2}} \\
\ln \Lambda_{a b}=\ln \left\{\left(\frac{m_{a} m_{b}}{m_{a}+m_{b}}\right) \frac{2 \alpha c \lambda_{D}}{e^{2}} \max \left[\left(\frac{2 \bar{E}_{a}}{m_{a}}\right)^{\frac{1}{2}},\left(\frac{2 \bar{E}_{b}}{m_{b}}\right)^{\frac{2}{2}}\right]\right\}-\frac{1}{2} .
\end{gathered}
$$

Here, $Z_{\zeta}$ is the charge number of particle $\zeta, e$ is the charge of an electron, $c$ is the speed of light in a vacuum, $\lambda_{D}$ is the Debye length, $\alpha$ is the fine structure constant, $\bar{E}_{\zeta}$ is the mean energy of particles $\zeta$ and the summations in equation 1.13 and equation 1.14 are over all other charged species.

Solving for the Fokker-Planck collision operator in terms of the full Rosenbluth Potentials is beyond the scope of this work. The emphasis of this dissertation is to present new numerical methods for solving the Fokker-Planck type of equation. Therefore, a simplified collision operator is used. To ensure that enough of the correct physics is retained, a simplified collision operator is required to have three properties [6],

1. It must conserve the number of particles or mass.

2. It must have both frictional and diffusional properties.

3. It must have no effect on a Maxwellian distribution. 
The following simplified Fokker-Planck collision operator satisfies all three $[7,8]$,

$$
\left(\frac{\partial f}{\partial t}\right)_{c}=\nu_{c} \frac{\partial}{\partial v}\left\{(\mathbf{v}-\bar{v}) f+\left[\frac{k T}{m}\right] \frac{\partial f}{\partial v}\right\}
$$

where, $T$ is the fluid temperature, $k$ is the Boltzmann constant, and $\bar{V}$ is the fluid velocity. To verify the first requirement one integrates the collision operator from minus infinity to infinity and uses the fundamental theorem of calculus,

$$
\int_{-\infty}^{\infty} \nu_{c} \frac{\partial}{\partial \mathrm{v}}\left\{(\mathrm{v}-\overline{\mathrm{V}}) f+\left[\frac{k T}{m}\right] \frac{\partial f}{\partial \mathrm{v}}\right\} d(\mathrm{v})=\left.\nu_{c}\left\{(\mathrm{v}-\overline{\mathrm{v}}) f+\left[\frac{k T}{m}\right] \frac{\partial f}{\partial \mathrm{v}}\right\}\right|_{-\infty} ^{\infty}=0
$$

Both $f$ and $\frac{\partial f}{\partial \mathrm{v}}$ approach zero as $\mathrm{v} \rightarrow \infty$. It should be noted that it is important to enforce this boundary condition in the numerical implementation as well. The second requirement is satisfied since equation 1.17 has the same form as equation 1.11. The proof of the third requirement is more detailed and is shown in Section 3.5.3.

The previous discussion has been heuristic because the full derivation of the collision operator used in this dissertation was done by Catto $[7,8]$ and not the author. The derivation of a similar collision operator was presented by Braginskii in 1957 [9]. Braginskii started his derivation from work by Landau in 1937 [10]. His goal was to derive a simplified collision operator that represented collisions between ions and electrons. In this derivation he made use of the fact that since electrons were much lighter than ions, the velocities of the electrons would be much higher than the velocity of the ions for comparable temperature, or collisional equilibrium. This allowed him to simplify the collision operator in three ways.

1. Since the ion velocity was small, equations could be expanded in terms of the ion velocity and higher order terms neglected. 
2. Since the ion velocity is very small relative to the electron velocity, equations could be expanded in terms of the ratio of ion velocity to electron velocity and higher order terms ignored.

3. Since the ion velocity is small compared to the electron velocity, the relative velocity between ions and electrons can be assumed to be the electron velocity.

He then made two more assumptions. He assumed that the electron distribution function was spherically symmetric. He also had to assume that the difference between the mean velocities of the ions and electrons was small relative to the electron velocities. With these assumptions, he was able to derive a collision operator which had the same structure as Brownian particles in a moving medium. His collision operator has the form,

$$
\left(\frac{\partial f}{\partial t}\right)_{c}=A \frac{\partial}{\partial v}\left\{B f+C \frac{\partial f}{\partial v}\right\}
$$

which is the same form as equation 1.17.

\subsection{Why Kinetic Modeling?}

Kinetic modeling of plasma particle interactions is required whenever the particle mean-freepath (mfp) is on the order of the scale length of the geometry of interest. Such relatively large mfp's can be caused by a variety of physics. Large mfp's are a result of low collision frequencies. An example of the Coulomb collision frequency written in terms of the plasma number density and temperature is given by Book [11],

$$
\nu=K_{1} \frac{n}{T^{3 / 2}}
$$


where $\nu$ is the collision frequency, $n$ is the number density, $T$ is the temperature, and $K_{1}$ is a constant. From equation 1.20, one can see that the collision frequency scales with the number density and the inverse of the temperature to a power. Therefore, low collision frequencies can be obtained from low number densities or high temperatures.

Chen [12] derives the collision frequency from a single particle perspective. With this derivation the collision frequency now depends on the particle velocity and has the form,

$$
\nu=K_{2} \frac{n}{v^{3}},
$$

where $v$ is the particle velocity and $K_{2}$ is a constant. Given this form of the collision frequency, another cause of large mfp's is when a significant fraction of the particles are moving at a high velocity. From equation 1.21 one can see that the collision frequency scales with the inverse of the particle velocity cubed, so "fast" particles can move large distances without colliding.

A third reason for using kinetic modeling is when the length scales of interest are very small. This usually occurs in modelling a plasma when there is a boundary that needs to be accurately resolved. The short geometric length scales can also result from steep gradients away from a boundary that need to be resolved (i.e., a shock-like structure).

Examples of applications that may require kinetic modeling include: inertial confinement fusion (ICF) [13]; plasma processing [14-16]; modeling of the boundary layer plasma in a tokamak fusion reactor $[17,18]$; and excimer lasers [19].

There are times in inertial confinement fusion when the Spitzer conductivity over-predicts the actual thermal conductivity in the plasma. Spitzer conductivity is derived assuming that 
the distribution is only slightly non-Maxwellian and has the following form [20,21],

$$
\kappa_{s}=20\left(\frac{2}{\pi}\right)^{\frac{3}{2}} \frac{(k T)^{\frac{5}{2}} k}{m_{e}^{\frac{1}{2}} e^{4} Z \ln \Lambda}
$$

where $\kappa_{s}$ is the Spitzer thermal conductivity and,

$$
\ln \Lambda=\ln \left[\frac{3}{2 Z^{2} e^{3}}\left(\frac{k^{3} T^{3}}{\pi n_{e}}\right)^{\frac{1}{2}}\right]
$$

is the maximum impact parameter. This over prediction of the thermal conductivity is caused by a departure from a Maxwellian distribution of the "fast" electrons when the length scales of interest are on the order of the electron mean free path. When this occurs, "flux limiting" is used. In this context "flux limiting" means that a correction is added to the transport equation to correct the flux predicted by the equation $[22,23]$. For example the classical heat flux equation,

$$
Q=-\kappa_{s} \nabla T
$$

is replaced with;

$$
Q=-\left[l(n k T)\left(\frac{k T}{m}\right)^{\frac{1}{2}}\left|\frac{1}{\nabla T}\right|\right] \nabla T
$$

where $Q$ is the heat flux and $l$ is called the "flux limit". Kinetic calculations, requiring no assumptions about the distribution functions, can be used to correctly set the "flux limit" value.

Three reasons that plasma processing applications may require kinetic modeling are steep gradients, small geometric length scales, and low pressure. The chemical reaction rates need to be very accurately resolved to describe how the plasma effects the chemical reactions occuring 
during plasma processing. It should be noted that these reaction rates can be strongly effected by the non-Maxwellian behavior of the electrons. The length scales of interest in plasma processing may be very small, such as in plasma etching for silicon chips [16].

In excimer laser modeling, accurate distribution functions are required for resolution of the collisions and reaction rates to make accurate predictions with the models. Most models use simplified electron kinetic equations because the required resolution of the energy variable with correct solution techniques is difficult on current computers [19]. In this application the high resolution of the energy phase space is required to resolve the fine structure of the electron impact cross sections and to resolve the nonlinear behavior of the electron-electron collisions. Excimer laser modeling requires accurate description of many collision processes. Examples of these are elastic collisions, excitation, dissociation, ionization, and recombination.

This dissertation is concerned with developing an advanced solution algorithm for applications where the distribution functions can be non-Maxwellian. In each of the above mentioned applications there exists the need to resolve length scales on the order of the particle mean free path. Since the particles must travel a few mean free paths before collisions redistribute them to a local Maxwellian distribution, these applications may have significant regions that are non-Maxwellian and thus require kinetic modeling instead of fluid modeling.

In an attempt to apply fluid codes in this long mean free path regime, "flux limiting" procedures are applied in evaluating transport quantities such as heat flux. Kinetic simulations have been used to study the accuracy of such procedures $[17,24]$. In applications such as plasma processing, radiative divertors, and excimer lasers, the "chemical" reaction rates are an important quantity. Fluid codes use the Maxwellian-averaged values of the reaction 
rates,

$$
\langle\sigma v\rangle=\frac{1}{n} \int f \sigma v d v
$$

where,

$$
n=\int f d v
$$

and $f$ is assumed to be a Maxwellian distribution function. If the distributions are nonMaxwellian, using these reaction rates may produce significant errors. Kinetic simulation can be used to approximate potential errors in these reaction rates. Since kinetic modeling may be too expensive for large scale simulations, one approach is to use fluid models for the large scale applications that incorporate chemical reaction rates with transport coefficients derived from kinetic simulations. By modifying these coefficients in the fluid simulation code, one can better approximate the correct physics in areas where the distributions are non-Maxwellian. Another approach is to develop hybrid codes that contain both fluid and kinetic models [25].

\subsection{The Tokamak Edge Plasma}

This dissertation presents a fully implicit solution algorithm for the Vlasov-Fokker-Planck (VFP) equation and applies it to simplified kinetic models of the edge plasma in a tokamak fusion reactor. A tokamak is a "donut" shaped reactor as shown in figure 1.1. In a diverted tokamak, the core plasma and edge plasma are separated by the magnetic separatrix as shown in figure 1.2. As the plasma diffuses radially across the tokamak separatrix, it moves from a region of closed magnetic field lines, "the core", to a region of open magnetic field lines, "the edge". These open magnetic field lines guide the plasma to the divertor plates. For some 


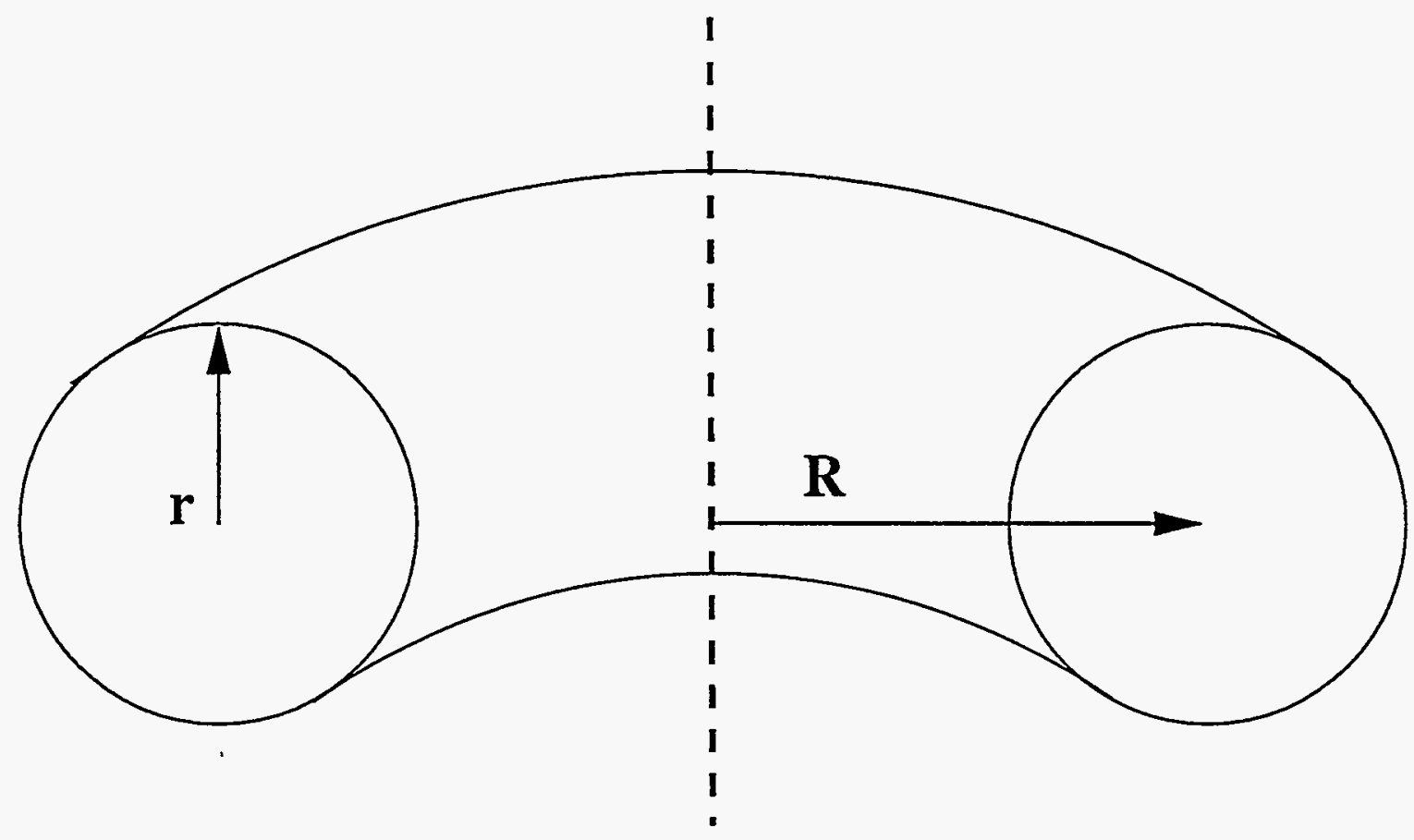

Figure 1.1: Tokamak Geometry

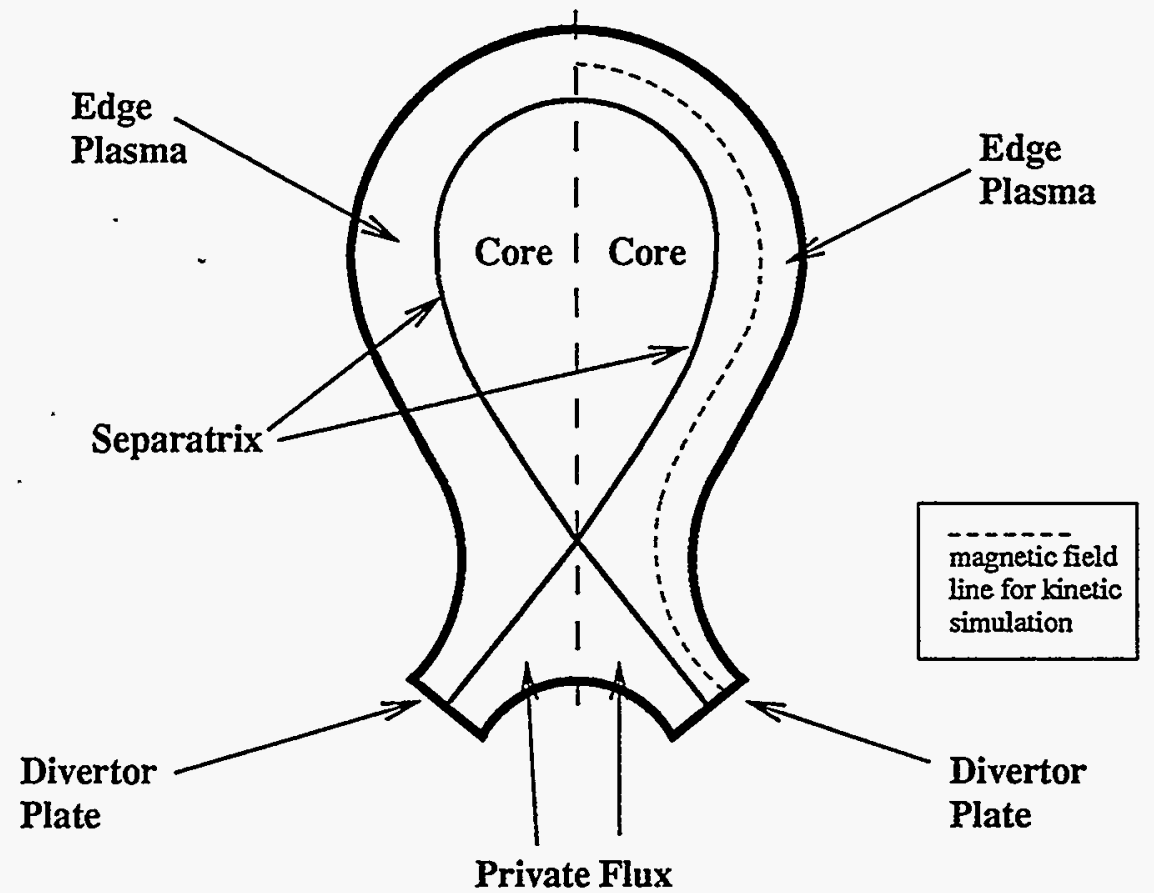

Figure 1.2: Tokamak Cross Section 
edge plasma conditions, the particle mean free path due to Coulomb collisions, which scale as $\frac{n}{T^{\frac{3}{2}}}$, may be on the same order as the problem geometry. This similarity in scale between mean free path and fluid transport length motivates a kinetic treatment $[17,24,26-30]$.

There has been extensive work done on the modeling of the edge plasma using fluid equations [18,31-33]. This work is based on the equation set from Braginskii which was derived from the Vlasov-Fokker-Planck equations.

The equations are derived by first linearizing the VFP equation by,

$$
f=f_{0}+f_{1} \text {. }
$$

Here $f_{0}$ is the Maxwellian part of the distribution function and $f_{1}$ is a small perturbation from Maxwellian. This linearization is substituted into the VFP equation and only first order terms (in $f_{1}$ ) are retained. It should be noted that resistivity, viscosity, conductivity, plasma current and heat flux are all functions of $f_{1}$, which is proportional to gradients of temperature and potential. The Braginskii equations [2] are then obtained by taking the first three moments of the now linear (in $f_{1}$ ) VFP equation. The linearization used to derive the Braginskii equations breaks down if the ratio of the collisional mean-free-path (mfp) to the temperature length scale is greater than $1 / 10$ [29] that is,

$$
\frac{\lambda_{c}}{L_{T}}>\frac{1}{10}
$$

where $\lambda_{c}$ is the collisional mfp given by,

$$
\lambda_{c}=\frac{v_{t h}}{\nu}
$$


where $\nu$ is the collision frequency and $v_{t h}$ is the thermal velocity given by,

$$
v_{t h}=\left(\frac{2 k T}{m}\right)^{\frac{1}{2}}
$$

Here, $L_{T}$ is the temperature length scale given by,

$$
L_{T}=\frac{1}{\left(\frac{1}{T}\right)\left(\frac{\partial T}{\partial x}\right)}
$$

If this condition is approached or exceeded, then kinetic models should be employed or fluid equations which keep second order and higher terms in $f$ should be used.

There are three areas where kinetic modeling may be important for tokamak edge plasma modeling. The first is in calculating the correct heat flux to the divertor plate [18]. As the plasma interacts with the plate a sheath is formed and the physics inside of the sheath produces non-Maxwellian distribution functions. Since the fluid codes are using Maxwellianaveraged values to compute heat flux in a non-Maxwellian region, there are errors being introduced. If the distributions are highly non-Maxwellian, the errors may be large. The second area relates to the excitation and ionization of the neutrals coming off the divertor plate. These reaction rates, $\langle\sigma v\rangle$, are calculated assuming a Maxwellian distribution which is not correct in areas with very large gradients in temperature or density. The third involves computing the correct heat flux along a magnetic field line where the temperature is high and the number density is low. In this situation the collisional mfp is large and fluid codes tend to over-predict the heat flux. 


\subsection{General Numerical Approach}

The numerical approach chosen for this work was a fully implicit discretization of the VlasovFokker-Planck equation on a velocity-spatial grid. This approach is in contrast to another popular method which is the combination PIC-Monte Carlo method [24]. The PIC method (particle in cell) was defined earlier in Section 1.1. The Monte Carlo method is a statistical method to simulate the Coulomb collisions and the charged/neutral particle interactions. This is accomplished by scattering colliding particles through an angle $\theta$, where $\theta$ is chosen randomly from a global collision frequency. The deterministic approach of discretizing the Vlasov-Fokker-Planck Equation has the advantage of not incorporating statistical effects in the solution. Therefore, when the solution is converged on the grid to the desired convergence tolerance, the simulation is done. With the PIC-Monte Carlo methods, the solution that is reached is limited and may be repeated with more particles to improve accuracy. However, the first drawback to the deterministic approach is the difficulty in resolving the velocity dimension over its entire range from large negative to large positive velocities. This can be accomplished by setting a limit for the maximum particle velocity. The distribution function falls off like $e^{-v^{2}}$. Thus for large enough velocities, $f(v)$ is approximately zero. This places a great importance on the discretization techniques chosen. The second drawback is that numerical dissipation, caused by the first order operators, can be a serious problem, as shown later in Section 4.2. As will be seen in Chapter 3, discretization of both the first and second order operators in the Fokker-Planck collision operator is also very important. Some of the velocity space discretization error can be minimized by the use of a non-uniform velocity grid.

An important consideration in choosing the numerical method is the level of implicitness 
of the solution technique. This is simply the amount of information that is included at the "new" time step in the equation or equivalently the number of terms included in the advancement matrix. The simplest approach is to integrate the VFP equation by an explicit method. Here, all information is at old time and the matrix, which is a vector multiple of the identity matrix, is easily inverted. The drawback to this approach is the time step restrictions required for numerical stability. Since these restrictions are so tight, a very large number of iterations must be used (PIC codes suffer from similar time step restrictions). The most popular semi-implicit method for integrating the VFP equation is the Alternating Direction Implicit (ADI) Technique [34]. In this method, part of the information is at "new" time and part is at "old" time. Then the parts at "new" time and "old" time are switched. This is iterated until a desired convergence tolerance is reached. This method is popular since it requires very little storage of data, hence problems can be run on small computational platforms. Only small banded matrices are solved due to the choice of what is "new" and what is "old" information.

A fully implicit approach has the advantage of not having a linear numerical stabilitybased time step requirement. However, theré may be accuracy or non-linear stability restrictions that govern the size of the time step that can be used. This approach has had limited use in the past [35]. It uses a very dense matrix, caused by the integral operators, with large memory requirements, hence it has not been fully explored. As discussed in following sections, there are numerical methods that minimize the memory requirements while still keeping the implicit coupling of the integral operators. 


\subsection{Previous Work}

The previous work in this area can be broken into to two general groups; statistical and deterministic. The statistical work consists of the PIC-based simulations. The PIC method advances the Vlasov equation, then the electric field calculations are computed based on cell averages, subsequently the Fokker-Planck operator is approximated by a Monte-Carlo approach.

\subsubsection{Statistical}

- Procassini, Birdsall, and Cohen [24] give a good example of statistical work. Their model has one spatial dimension parallel to the magnetic field, $x$, and two velocity components, $v_{\perp}$ and $v_{\|}$. This solution would be characterized as one spatial dimension, two velocity dimension, (1D2V). The particle equations of motion result in a set of ordinary differential equations (ODE's) that are integrated using the direct implicit method. For the Vlasov operator, the electric field is calculated by solving Poisson's equation via the direct implicit form of Poisson's equation. The Coulomb collisions are simulated using a Monte Carlo method.

This work also allows the inclusion of models for charge/neutral collisions. In this paper, Procassini et al. present results that solve for ions and electrons with the PIC algorithm, and a simple model for neutrals,

$$
n_{n}(z)=\hat{n}_{n} \exp \left(\frac{-[L-x]}{\lambda_{n}}\right)
$$

where $\hat{n}_{n}$ is the peak neutral density at the plate and $\lambda_{n}$ is the ionization mean-free-path. For this work, the sheath is not calculated but is included as a logical boundary condition that reflects the proper number of electrons to maintain a zero net current at the surface. A 
variety of results are presented for variations in the amount of recycling of neutrals off the divertor plate. This work was continued in 1991 by Procassini and Birdsall [29]. In this work the same code and models were used, but this time the effects of the collision rate on the transport quantities were studied.

Batischev, Krasheninnikov, Sigmar, Sigov, and Soboleva [26] provide another example of the statistical approach. In this work, three and four dimensional simulations are presented. The three dimension case is one-spatial and two-velocity, $1 \mathrm{D2V}$, either $\left(v_{\|}, v_{\perp}\right)$ or $(v, \theta)$, while the four dimensional work is two-spatial and two-velocity, 2D2V. The $1 \mathrm{D2V}$ work is collisional while the $2 \mathrm{D} 2 \mathrm{~V}$ is collisionless, Vlasov operator only. In this work, the electric field is calculated both by a quasi-neutrality condition and by Poisson's equation. In the quasi-neutral electric field calculation the electric field is calculated from assuming $n_{i}=n_{e}$, and by differencing the ion and electron momentum equations and solving for $E$. The electric field equation follows.

$$
E=-\frac{\left(\frac{\partial\left(\Pi_{e}-\Pi_{i}\right)}{\partial x}+F_{f r e}-F_{f r i}\right)}{2 e n}
$$

where $\Pi_{\zeta}$ is the stress tensor and $F_{f r \zeta}$ is the frictional force for species $\zeta$. For more details see Section 5.2. The Poisson equation was given as:

$$
\nabla E=\frac{1}{\epsilon_{0}} \sum_{\zeta=i, e} q_{\zeta} \int f_{\zeta} d(\mathrm{v})
$$

The PIC method is used for the Vlasov operator and the Fokker-Planck operator is solved using the Rosenbluth potentials. In earlier work from 1992 Krasheninnikov et al. [36] presented results from both a PIC code and a finite difference based Vlasov-Fokker-Planck code. For this work, both calculations are three dimensional $1 \mathrm{D2V}$. In all of this work, both ions 
and electrons are computed kineticly.

\subsubsection{Deterministic}

McCullen, Montierth, Morse, and Neuman [28] provide a good example of the deterministic approach. For this work, the electron distribution is assumed to be Maxwell-Boltzmann on the computational domain and therefore only an ion transport equation needs to be solved. Their calculations are both two dimension, one-spatial and one-parallel velocity, 1D1V $\left(x, v_{\|}\right)$ and three-dimensional, one-spatial and one-parallel velocity and one-perpendicular velocity, 1D2V $\left(x, v_{\|}, v_{\perp}\right)$. The electric field is calculated by solving Poisson's equation,

$$
\frac{d^{2} \phi}{d x^{2}}=-\frac{1}{\epsilon_{0}}\left(\sum_{\zeta} q_{\zeta} n_{\zeta}-e n_{e}(x, \phi)\right)
$$

where

$$
n_{e}(x, \phi)=n_{e 0} \exp \left(\frac{e \phi(x)}{k T_{e}}\right)
$$

and the effect of neutrals being re-emitted from the surface is included. Here $\phi$ is the electric potential. For this work, the Coulomb collisions are neglected and only collisions with neutrals are considered. The equations are integrated by a method of characteristics approach.

Later that same year, Montierth, Morse, and Neuman [17] presented results incorporating the Coulomb collisions. For this work a full Fokker-Planck operator in three-dimensions, one spatial dimension in the parallel direction, one-parallel velocity, and one-perpendicular velocity, 1D2V $\left(x, v_{\|}, v_{\perp}\right)$, was used to model ion-ion collisions. For this work the method of characteristics was abandoned for a conservative finite difference scheme.

Another addition to the literature in this area is the work of Sauter, Harvey, and Hin- 
ton [37]. The authors make a three-dimensional nodalization of the VFP equation, onespatial dimension parallel to the magnetic field lines and two-velocity dimensions (speed and angle),1D2V $(x, v, \theta)$. The electric field is computed either by Poisson's equation or by a quasi-neutrality condition (i.e., They take the appropriate moments of the VFP equation and then solve for E). They modelled the collisions with a complete Fokker-Planck operator based on the Rosenbluth potentials which are computed using a Legendre decomposition. The authors use an alternating direction implicit method (ADI) to integrate their finite difference formula. In this paper, the authors compute electron transport for a wide range of temperatures, from $10 \mathrm{eV}$ to $10 \mathrm{keV}$, and number densities, from $10^{17} \mathrm{~m}^{-3}$ to $10^{20} \mathrm{~m}^{-3}$, which result in low collision rates, $10^{-4} s^{-1}$ to $10^{2} s^{-1}$ For these conditions, resistivities are computed and compared with theoretical results but no actual divertor simulations were presented.

Another recent dissertation in a similar area is that of Coster [38] in 1993. He solved the VFP equation in three dimensions, one-spatial dimension and two-velocity dimensions, $1 \mathrm{D} 2 \mathrm{~V}\left(x, v_{\|}, v_{\perp}\right)$, using a complete Fokker-Plank operator that was evaluated by solving for the Rosenbluth potentials. He used an ADI approach for integrating the Vlasov operator, but he lagged the electric field and Fokker-Planck operator at old time. The electric field was calculated from Poisson's equation. Results were presented for both electrons and electrons and ions for a zero-spatial two-velocity grid, $0 \mathrm{D2V}\left(v_{\|}, v_{\perp}\right)$. On this grid, he checked conservation of energy, inter-particle friction and thermal equilibrium of the two species. Results are also shown for one-spatial dimension and two-velocity dimensions. He presented results for both ion-electron calculations and three species, two-ion electron calculations. It should be noted that Coster did not include an ionization source due to neutral ionization, but this was because he only modelled the sheath. 
Another addition to the literature of this field is the work of Abou-Assaleh, Matte, Johnston, and Marchand [30]. Their work is based on the Fokker-Planck International (FPI) code. This code solves a three dimensional kinetic equation for the electrons, one-spatial and two velocity, $1 \mathrm{D2V}\left(x, v_{\|}, v_{\perp}\right)$, and fluid equations for the ions and the neutrals. The kinetic model contains collision operators for ion-electron and electron-electron Coulomb collisions, ionization, and excitation. Their approach is a hybrid kinetic fluid model that incorporates fast solutions for the ions and accurate solutions for the electrons, by iterating between solutions of the fluid and kinetic codes.

\subsection{Overview of Dissertation}

This dissertation presents information on the implementation of the Matrix-Free NewtonKrylov technique to the one-velocity one-spatial (1D1V) Vlasov-Fokker-Planck Equations for ions and electrons. This numerical technique allows for a fully implicit, fully coupled, solution to the VFP equation without incurring large memory requirements. This is made possible by taking advantage of the structure of the Newton-Krylov technique which allows for the matrix to be replaced with function evaluations. To obtain the desired accuracy on reasonably sized grids, higher order differencing was used on the Vlasov operator. However, unlike upwind differencing which is a low order monotonic discretization technique, the higher order methods are sometimes oscillatory. To prevent oscillations, (non-monotonic behavior), flux limiting techniques were employed. It should be noted that the same term flux limiting is used to describe two different numerical effects in this dissertation. When it is used to describe bounding a term in a transport equation it will be written "flux limiting" with quotations. For the rest of this dissertation it will be used to indicate a numerical discretization technique 
and it will not be placed in quotes, (flux limiting). The context that it is used in should help to clarify the difference between these two usages.

The emphasis of this dissertation is numerical in nature, with enough physics and application work to prove the value of the numerical method. Following this theme, the full three-velocity dimension Fokker-Planck collision operator was not solved. The collision operator chosen was a simplified one-velocity version of the Fokker-Planck collision operator (FPCO). The simplified operator maintains both the diffusive and convective nature of the FPCO, but doesn't require the solution of equation 1.5 or equation 1.6 or the Rosenbluth potentials. Therefore, the flavor of the correct physics is included without incorporating the coding complexity of the full FPCO.

Much of the earlier work with the VFP equation had an imposed electric field or used Poisson's equation to solve for the electric field. In this dissertation the electric field is determined by taking the second moment of the kinetic equations (i.e., the fluid momentum equations) for both ions and electrons and then back solving for the electric field. This quasineutral approach provides a self consistent definition of the electric field without adding the constraints caused by using Poisson's equation (for more detail see Section 5.3 of Chapter 5).

Because of the low dimensionality and the simplified Coulomb collision operator, additional species can be easily added to this code. Since the coupling between species is all incorporated through function evaluations, addition of other species has only a minor impact on the code structure and the numerical implementation. The independence of the species, indicates that a functional decomposition, (i.e., one species per $\mathrm{CPU}$ ), of the solution algorithm may lead to an efficient parallel implementation.

The contribution to the field from this dissertation is the introduction of a numerical 
method that allows for a higher level of implicit solution than was previously practical for the Vlasov-Fokker-Planck equation. This numerical method can be incorporated into other codes that have better physics due to higher dimensionality or more complete collision operator descriptions. Also, by choosing a two dimensional state space and a simplified collision operator, a code has been developed that runs efficiently on a modern workstation and can be rapidly applied to problems to determine if a more complete kinetic simulation is warranted.

The organization of the rest of the dissertation is as follows. Chapter 2 presents the results from one-spatial one-velocity ( $1 \mathrm{D} 1 \mathrm{~V})$, ion-only simulations that are used to demonstrate the numerical technique. Chapter 3 describes ion-electron solutions in a zero-spatial one-velocity (OD1V) grid. Here the collision operators are investigated in detail. Chapter 4 describes the higher order discretization of the Vlasov operator. Chapter 5 then combines all of the previous work to simulate plasma impinging on a wall, a simulation of the tokamak divertor plasma. In Chapter 6 the work is summarized, conclusions are drawn, and future work is suggested. 


\section{Chapter 2}

\section{One Spatial One Velocity (1D1V)}

\section{Ion Only}

\subsection{Introduction}

In this chapter the numerical technique Matrix-Free Newton Krylov (MFNK) is described and demonstrated on an ion-only problem in the simplest geometry. The test problem chosen simulates a simplified model of the ion transport in the edge plasma of a tokamak reactor. Results are shown that indicate the validity of the approach and the behavior of the simplified operators and models.

The organization of the rest of this chapter is as follows. Section 2.2 presents the mathematical model and the equations that are solved. Section 2.3 justifies the use of the MFNK method and describes the algorithm, with emphasis on Newton's method, the Krylov method, and the use of the matrix-free approximation. Section 2.4 describes the model problem and shows calculational results, and Section 2.5 summarizes the chapter. 


\subsection{Mathematical Model}

The model equation in this chapter contains both the Vlasov operator and a simplified FokkerPlanck collision operator. In general form, the time dependent Vlasov-Fokker-Planck equation has seven independent variables: three velocity dimensions, three spatial dimensions, and one temporal dimension. Equation 1.3 is repeated here for clarity,

$$
\frac{\partial f}{\partial t}+\mathbf{v} \cdot \nabla f+\frac{q}{m}(\mathbf{E}+\mathbf{v} \times \mathbf{B}) \cdot \frac{\partial f}{\partial \mathbf{v}}=\left(\frac{\partial f}{\partial t}\right)_{c}
$$

The model problem chosen simulates ion transport along magnetic field lines in the edge plasma of a tokamak. Assuming toroidal symmetry, the steady state problem can be reduced to four dimensions, two spatial and two velocity, or $2 \mathrm{D} 2 \mathrm{~V}$. To demonstrate the numerical method, it is applied to a simplified two-dimensional, or 1D1V, model equation. This equation is derived from a more complete three-dimensional, 1D2V, Vlasov-Fokker-Planck equation for transport along a field line $[17,28]$, in which the two velocity coordinates are perpendicular, $v_{\perp}$, and parallel, $v_{\|}$, to the magnetic field lines.

For the model problem, the perpendicular velocity dimension, $v_{\perp}$, is eliminated by assuming it to be Maxwellian [7]. More formally,

$$
f\left(v, v_{\perp}\right)=\left(\frac{m}{2 k T}\right) \exp \left(\frac{-m v_{\perp}^{2}}{2 k T}\right) F(v)
$$

With this assumption, the distribution function has the form $F(x, v, t)$ were $x$ is the distance along the magnetic field line and $v$ is just $v_{\|}$. Therefore, the two dimensional time-dependent 
ion transport equation is as follows:

$$
\frac{\partial F}{\partial t}+v \frac{\partial F}{\partial x}+\left(\frac{q E}{m}\right) \frac{\partial F}{\partial v}=\left(\frac{\partial F}{\partial t}\right)_{c}+\left(\frac{\partial F}{\partial t}\right)_{i n z}+\left(\frac{\partial F}{\partial t}\right)_{s r c}
$$

where $q$ is the charge on the ion, $m$ is the mass of the ion, $v$ is the particle velocity along the magnetic field line, and $E$ is the electric field along the magnetic field line. There are two new source terms include in equation 2.3 that where not included in equation $1.3,\left(\frac{\partial F}{\partial t}\right)_{i n z}$ and $\left(\frac{\partial F}{\partial t}\right)_{s r c}$. The $\left(\frac{\partial F}{\partial t}\right)_{i n z}$ term is a source term which accounts for the ions which are created by ionization of the neutral particles coming off the divertor plate. The $\left(\frac{\partial F}{\partial t}\right)_{s r c}$ term represents the source of ions which diffuse across the closed magnetic field lines, the core of a tokamak, into the open magnetic field lines, the edge plasma of the tokamak, (see figure 1.2 for details).

The distribution function moments are defined by,

$$
\begin{aligned}
n & \equiv \int_{-\infty}^{\infty} F d v \\
n \bar{V} & \equiv \int_{-\infty}^{\infty} v F d v \\
n k T & \equiv m \int_{-\infty}^{\infty}(v-\bar{V})^{2} F d v
\end{aligned}
$$

where $n$ is the particle density, $\bar{V}$ is the fluid velocity and $T$ is the fluid temperature.

Finally the equation set is closed with an equation of state

$$
P=n k T .
$$




\subsubsection{Fokker-Planck Collision Operator}

For a complete Fokker-Planck representation of the collision operator one would solve the Rosenbluth potentials [4] and then use them to express the Fokker-Plank collision operator. Since the motivation of this chapter is to demonstrate and study the numerical methods, a simplified Fokker-Planck collision operator $[7,8,39,6,40]$ was chosen,

$$
\left(\frac{\partial F}{\partial t}\right)_{c}=\nu_{i i}\left\{\left[\frac{k T}{m}\right] \frac{\partial^{2} F}{\partial v^{2}}+\frac{\partial[(v-\bar{V}) F]}{\partial v}\right\}
$$

where $\nu_{i i}$ is the ion-ion collisional frequency. For more information see Section 1.2.

\subsubsection{Neutral Ionization Model}

The model for the neutral ionization source term, $\left(\frac{\partial F}{\partial t}\right)_{i n z}$, is analogous to the neutron diffusion approximation [41]. The first moment of the neutral particle transport equation, the continuity equation, is given by Vold et. al. [42] as,

$$
\frac{\partial n_{0}}{\partial t}+\frac{\partial \Gamma_{n 0}}{\partial x}=-n n_{0}\langle\sigma v\rangle_{e z}
$$

where $n_{0}$ is the number density of the neutral particles, $\Gamma_{n 0}$ is the neutral particle flux, and $\langle\sigma v\rangle_{e z}$ is the Maxwellian-averaged (see equation 1.26) ionization rate between electrons and neutral particles with $\sigma$ representing the cross section for that interaction (the subscript ez stands for electron ionization). From the second moment of the neutral particle transport equation, a relationship can be obtained for $\Gamma_{n 0}$. If a Legendre polynomial expansion is used 
retaining only terms of order $\mathrm{P} 1$, the following equation for $\Gamma_{n 0}$ results,

$$
\frac{1}{u_{0}} \frac{\partial \Gamma_{n 0}}{\partial t}=\frac{1}{3} \frac{\partial n_{0} u_{0}}{\partial x}+\Sigma_{t r} \Gamma_{n 0}
$$

where $u_{0}$ is the neutral thermal velocity and $\Sigma_{t r}$ is the macroscopic transport cross section. If the time rate of change of $\Gamma_{n 0}$ is assumed small, and the thermal velocity of neutrals is assumed independent of $x$, then the following relation for $\Gamma_{n 0}$ results,

$$
\Gamma_{n 0}=\frac{-u_{0}}{3 \Sigma_{t r}} \frac{\partial n_{0}}{\partial x}
$$

Vold et. al. [42] presented the following relation for $\Sigma_{t r}$,

$$
\frac{1}{\Sigma_{t r}} \equiv \lambda_{t r}=\frac{u_{0}}{n\left(\langle\sigma v\rangle_{e z}+\langle\sigma v\rangle_{c z}\right)}
$$

where $\langle\sigma v\rangle_{c x}$ is the Maxwellian-averaged charge exchange rate (the subscript $c x$ stands for charge exchange). Knoll et. al. [31] gave the following relations for the reaction rates for a deuterium plasma, typical of the tokamak divertor region,

$$
\begin{aligned}
\langle\sigma v\rangle_{c x} & =2.0 \times 10^{-14} \mathrm{~m}^{3} / \mathrm{s} \\
\langle\sigma v\rangle_{e z} & =\frac{3.0 \times 10^{-14} a^{2}}{3.0+a^{2}} \mathrm{~m}^{3} / \mathrm{s} \\
a & =\frac{T_{e}(e V)}{10}
\end{aligned}
$$


where $T_{e}$ is the electron temperature in units of electron volts. With the above definitions, $\Gamma_{n 0}$ can be defined as follows:

$$
\Gamma_{n 0}=\frac{-u_{0}^{2}}{3 n\left(\langle\sigma v\rangle_{e z}+\langle\sigma v\rangle_{c x}\right)} \frac{\partial n_{0}}{\partial x}
$$

If $n$ is assumed independent of $x$, equation 2.9 can be rewritten as,

$$
\frac{\partial n_{0}}{\partial t}-\frac{u_{0}^{2}}{3 n\left(\langle\sigma v\rangle_{e z}+\langle\sigma v\rangle_{c x}\right)} \frac{\partial^{2} n_{0}}{\partial x^{2}}=-n n_{0}\langle\sigma v\rangle_{e z}
$$

In steady state the following equation results:

$$
\frac{\partial^{2} n_{0}}{\partial x^{2}}-\left[\frac{3 n^{2}\langle\sigma v\rangle_{e z}\left(\langle\sigma v\rangle_{e z}+\langle\sigma v\rangle_{c x}\right)}{u_{0}^{2}}\right] n_{0}=0
$$

If one defines,

$$
\lambda_{n}=\left[\frac{u_{0}^{2}}{3 n^{2}\langle\sigma v\rangle_{e z}\left(\langle\sigma v\rangle_{e z}+\langle\sigma v\rangle_{c x}\right)}\right]^{1 / 2}
$$

then the following equation for $n_{0}$ results:

$$
\frac{\partial^{2} n_{0}}{\partial x^{2}}-\frac{1}{\lambda_{n}^{2}} n_{0}=0
$$

That equation has the following solution:

$$
n_{0}(x)=n_{0}(0) \exp \left(-x / \lambda_{n}\right)
$$

A representative value for $\lambda_{n}$ can be computed from equation 2.19 and equation 2.13 through equation 2.15. Assuming an electron temperature, $T_{e}=10 \mathrm{eV}$, a plasma density, $n=1 \times$ 
$10^{19} \mathrm{~m}^{-3}$, and a neutral thermal velocity, $u_{0}=3.5 \times 10^{4} \mathrm{~m} / \mathrm{s}$, one obtains $\lambda_{n}=.14 \mathrm{~m}$.

In a model problem of a high recycling divertor, a significant fraction of the plasma which strikes the divertor plate is recycled as neutral atoms. These neutrals then diffuse back into the plasma and are ionized by electron impact ionization. The model for the neutrals which are generated at the plate follows:

$$
f_{n}(x)=f_{n}(L) \exp \left(\frac{-(x-L)}{\lambda_{n}}\right)
$$

Here $f_{n}$ is the neutral distribution function, $\lambda_{n}$ is the neutral mean free path to collisions with ions as described above, and $L$ is the spatial dimension of the region. The discrete form of the ionization term for these calculations is

$$
\left(\frac{\partial F}{\partial t}\right)_{i n z}=\frac{\nu_{n} \delta\left(v-v_{n}\right) f_{n}}{\Delta v}
$$

where $\nu_{n}=v_{n} / \lambda_{n}$ is the electron impact ionization frequency, and $\Delta v$ is the mesh spacing in the $v$ direction. This assumes that the neutral particle distribution is a beam with velocity $v_{n}$. Simulations are presented in this chapter and in Chapter 5 , with $f_{n}$ assumed to be a Maxwellian distribution in velocity space.

\subsubsection{Core Source}

The core source term is based on work from Emmert [43] and has the form,

$$
\left(\frac{\partial F}{\partial t}\right)_{s r c}=\left(\frac{1}{\Delta v}\right)\left(\frac{R_{i n j} h(x) v m}{2 k T_{i n j}}\right) \exp \left[\frac{m(v-\bar{V})^{2}}{2 k T_{i n j}}\right]
$$


where $R_{i n j}$ is the particle injection rate and $h(x)$ is the injection shape factor, such that,

$$
\int_{0}^{L_{i n j}} h(x) d x=1
$$

where $L_{i n j}$ is the spatial dimension of the injection region, and $T_{i n j}$ is the temperature of the injected particles. This source produces a Maxwellian distribution in the absence of an electric field. It may not be the most appropriate source model for a collisional plasma and other models are investigated in Chapter 5. In this model problem, the particle source comes mainly from neutral particles recycling off the divertor plate and being ionized, and the core source is used primarily for energy, i.e., to define a temperature.

\subsubsection{Electric Field}

For this single species analysis, a simplified form of the electric field calculation has been

used. Taking the $m v$ moment of equation 2.3 , ignoring $\left(\frac{\partial F}{\partial t}\right)_{c},\left(\frac{\partial F}{\partial t}\right)_{i n z}$, and $\left(\frac{\partial F}{\partial t}\right)_{s r c}$, yields an ion momentum equation of the following form:

$$
m_{i} \frac{\partial n_{i} \bar{V}_{i}^{2}}{\partial x}=-\frac{\partial P_{i}}{\partial x}+e n_{i} E
$$

Similarly for the plasma electrons:

$$
m_{e} \frac{\partial n_{e} \bar{V}_{e}^{2}}{\partial x}=-\frac{\partial P_{e}}{\partial x}-e n_{e} E
$$

where $P_{\zeta}$ is the fluid pressure of species $\zeta$. Since $m_{i}>m_{e}$, the left hand side of equation 2.27 can be set to zero. If it is assumed that $P_{i}=P_{e}=P$ and $n_{i}=n_{e}=n$, then the following 
form of the electric field results,

$$
E=-\frac{1}{e n} \frac{\partial P}{\partial x}
$$

where $e$ is the charge of an electron. Again a simplified model has been used to demonstrate the numerical method. For more information on the electric field see Section 5.3.

\subsection{Solution Algorithm}

\subsubsection{Fully Implicit Motivation}

Given the above equations and the decision to use a deterministic solution method, one now has to decide on the level of implicitness for the numerical integration. These levels of implicitness can be categorized as explicit, semi-implicit, and fully implicit.

The explicit option is clearly the easiest to code. One simply has to code equation 2.3 with all variables evaluated at old time except for the $\frac{\partial F}{\partial t}$ term. The explicit method requires no matrix inversion, so it is not as computationally complex as the other more implicit methods. The drawback to this approach comes from numerical stability. Because of the potential for very short time scales when electrons are considered, very small time steps would have to be taken. Therefore, many time steps would be required to reach steady state, or to even resolve a realistic transient.

Consider the Courant stability limit from Roach [44] for explicit first order convective derivatives like $v \frac{\partial f}{\partial x}$,

$$
\frac{v \Delta t}{\Delta x}<1
$$

Here, $\Delta t$ is the numerical time step and $\Delta x$ is the numerical spatial step. Choosing numbers from Table 2.1 one can compute a representative time step. The spatial step is given 
approximately by $\Delta x=L / n x=0.06 \mathrm{~m}$. The worst case velocity is $v=v_{\max }=1.5 \times 10^{5} \mathrm{~m} / \mathrm{s}$. Now these values can be substituted into equation 2.29 and $\Delta t$ can be solved for resulting in $\Delta t<\Delta x / v=4.0 \times 10^{-7} \mathrm{~s}$. This is an incredibly restrictive time step.

A second approach is semi-implicit or alternating direction implicit (ADI) [34]. In this method, implicit coupling occurs along a given spatial dimension, " $x$ " strip, or velocity dimension, " $v$ " strip. Implicitness along a strip results in having to solve simple banded matrices and many "canned" packages exist for solving banded matrices. The solution proceeds by first sweeping over all " $v$ " strips then sweeping over all " $x$ " strips. This ADI iteration is continued until the solution converges $[5,17,28,37]$. The drawback to this approach is that for problems with important integral coupling like the electric field, convergence of the ADI algorithm may be slow because the integral coupling is not included implicitly. Recall that the electric field is represented as a gradient of the fluid pressure, and the fluid pressure is calculated from the equation of state, equation 2.7 , and an integral moment of the distribution function, equation 2.6 .

Also, convergence of ADI methods may be slow for equations which contain multiple time scales [34]. For example, in an edge plasma simulation near the core, the temperature may be $100 \mathrm{eV}$ with an ion number density of $10^{19} \mathrm{~m}^{-3}$, while near the divertor plate one may have $1 \mathrm{eV}$ and $10^{21} \mathrm{~m}^{-3}$. For this example the coupling near the core would be tightest in the " $x$ " direction where the Vlasov streaming operator dominates, but near the plate, coupling would be tightest in the " $v$ " direction since the Fokker-Planck collision operator would be dominating there. The ratio of Coulomb collisions from the core to the plate would be $10^{-5}$ based on the collisional frequency scaling as $n / T^{3 / 2}$.

A third approach, and the one chosen for this dissertation, is fully implicit. All variables 
are evaluated at new time and solved simultaneously. Because the equations are non-linear, Newton's method is used to linearize the problem. The resulting linear systems can be very large and dense (for a 100 velocity by 50 spatial grid there are $7.549 \times 10^{5}$ non-zero elements in the solution matrix out of $2.5 \times 10^{7}$ total entries, or a $3 \%$ fill rate). The density of the matrix results from the integral coupling due to the moment quantities that appear as coefficients of the derivative terms. The storage of this large dense matrix makes most interesting applications prohibitively expensive in terms of memory.

The matrix storage problem can be dealt with by evaluating the integral quantities at previous iteration values during the iterations. However, as the integral terms, $E$ and $\left(\frac{\partial F}{\partial t}\right)_{c}$, become important, this lagging of the integral quantities can significantly effect convergence, as will be seen later in Section 2.4. What is needed is a method that does not require the storage of the large dense matrix, but still has all of its effects during all the iteration steps. Matrix-Free Newton Krylov (MFNK) is one such method, and the method chosen in this dissertation.

\subsubsection{Newton's Method}

The nonlinearity of the problem comes from the electric field and the $\left(\frac{\partial F}{\partial t}\right)_{c}$ term in equation 2.3. To deal with this nonlinearity Newton's method is used. The main advantage of Newton's method is its local quadratic convergence, (i.e., the error at iteration $K$ is the square of the error at iteration $\mathrm{K}-1$ ). This quadratic convergence occurs only when the guess is within the Newton radius of convergence, which is a drawback to Newton's method [45]. One can get an initial guess within the radius of convergence by using a solution from a similar problem. Other methods for increasing the Newton radius of convergence are pseudotransients, damping strategies, and mesh sequencing [31,33]. These methods for improving 
Newton's method will be discussed in more detail in the following paragraphs.

Newton's method is a powerful technique for solving systems of nonlinear equations of the form,

$$
\mathrm{G}(\mathbf{y})=\left[g_{1}(\mathrm{y}), g_{2}(\mathrm{y}), \ldots, g_{n}(\mathbf{y})\right]^{T}=0
$$

where the state variable, $\mathbf{y}$, can be expressed as,

$$
\mathrm{y}=\left[\mathrm{y}_{1}, \mathrm{y}_{2}, \ldots, \mathrm{y}_{n}\right]^{T}
$$

Application of Newton's method requires the solution of the linear system,

$$
\mathrm{J}^{n} \delta \mathrm{y}^{n}=-\mathrm{G}\left(\mathrm{y}^{n}\right)
$$

where the elements of the Jacobian, $\mathrm{J}$, are defined by,

$$
\mathrm{J}_{i, j}^{n}=\frac{\partial g_{i}}{\partial \mathbf{y}_{j}^{n}}
$$

and the new solution approximation is obtained from,

$$
\mathrm{y}^{n+1}=\mathbf{y}^{n}+d \delta \mathbf{y}^{n}
$$

The constant, $d \in[0,1]$, in equation 2.34 is used to damp the Newton updates. The damping strategy is designed to prevent the calculation of non-physical variable values (i.e., negative distribution functions), and to scale large variable updates when the solution is far from the true solution. For example, $d$ is chosen such that, $f^{n+1}>0$ where $f^{n+1}=f^{n}+d \delta f$. This 

where tolcg is the convergence tolerance for the Krylov method. This results in increased efficiency by loosening the convergence tolerance when the nonlinear iteration is "far" from the solution (i.e., $\left\|\mathrm{G}\left(\mathrm{x}^{n}\right)\right\|$ is large) and high accuracy does not benefit the global convergence.

To increase the radius of convergence of Newton's method, a pseudo-transient is employed. In this method the Jacobian is modified as follows,

$$
\left(\frac{\mathbf{I}}{\Delta t}+\mathbf{J}^{n}\right) \delta \mathbf{y}^{n}=-\mathbf{G}\left(\mathbf{y}^{n}\right)
$$

where $I$ is the identity matrix. By increasing the diagonal of the Jacobian matrix, the size of the Newton update $\delta \mathrm{y}^{k}$ is lowered and the iteration is damped. Additionally, the increased diagonal will serve to decrease the condition number of the matrix and thus improve the performance of the Krylov method. However, by modifying the Jacobian, the solution will most likely require increased Newton iterations. In this implementation, the time step, $\Delta t$, is allowed to increase as the steady-state residual decreases.

Mesh sequencing is used to get an initial guess inside of the Newton radius of convergence by interpolating a converged solution from a coarse grid to a fine grid. Mesh sequencing is similar to the first upward pass of a multigrid " $V$ " cycle $[49,50]$. Using this technique places the largest number of Newton iterations on the coarse grids where the cost per iteration is lowest and the radius of convergence is largest.

\subsubsection{Krylov Method}

Krylov techniques are used as the iterative methou for solizing equation 2.32 . There are many Krylov, conjugate gradient-like, solvers [51,52], but for this dissertation, only tivo reneralized Minimal Residual (GMRES) method is used. This is because GMRES has been shown to be 
more robust in matrix-free implementations $[32,53,54]$ than other Krylov algorithms. Since the memory usage of the GMRES algorithm increases with each iteration, to achieve an efficient linear solution, one would want to use the GMRES algorithm when it only requires a small number of iterations. To lower the number of iterations, or in some cases to even get convergence, the original matrix system is preconditioned. Preconditioning reduces the spread and/or clusters eigenvalues of the matrix, both of which increase the performance of the Krylov method. In this dissertation, Incomplete Lower-Upper factorization [55], ILU(k) is used as the preconditioner.

\subsubsection{Matrix Free}

To solve equation 2.32 , the Krylov method only requires the action of the Jacobian in the form of matrix vector products, not the actual Jacobian matrix itself. Because of the large size and density of the Jacobian matrix, caused by the integral coupling, one would like to find a way to approximate the Jacobian's action [56,57]. This can be done using,

$$
J(y) \mathbf{u} \approx \frac{G(y+\epsilon \mathbf{u})-\mathrm{G}(\mathrm{y})}{\epsilon} .
$$

Here $u$ is a Krylov vector and $\epsilon$ is a small perturbation in state space. By using this approximation in the Krylov iteration, one is not required to explicitly form the Jacobian matrix on each Newton step, except for use in the preconditioner. Thus the large computational cost and storage of evaluating and storing each element in the Jacobian via equation 2.33 is replaced with one additional right-hand-side evaluation per GMRES iteration.

For this work, the preconditioner matrix used is a modified form of the Jacobian. Since the preconditioner need only be a "close" approximation to the true Jacobian, the preconditioner 
is constructed from the last iteration values for the integral quantities. Thus, only the differential operator is used in defining a preconditioner. Since the integral quantities are evaluated at the last iteration value, the preconditioner matrix has a penta-diagonal structure. This structure results from the five point stencil of the difference operators $\frac{\partial F}{\partial x}$ and $\frac{\partial F}{\partial v}$. Therefore, when ILU(0) is used as the preconditioner, the total preconditioner matrix storage is $5 \times N$ where $N$ is the total number of unknowns. As higher order techniques are employed in later chapters, this five point stencil will increase to a nine point stencil.

\subsection{Model Problem and Results}

To demonstrate the numerical method, a simple model problem was chosen. Figure 2.1 shows the geometry in $x, v$ space of the model problem. The model problem is a one-dimensional, along the magnetic field line, representation of a high recycling divertor (see figure 1.2). The left boundary is a symmetry plane in $x$, which is described by,

$$
F(0, v)=F(0,-v)
$$

The ions are injected in the source region according to equation 2.24 at a temperature of $75 \mathrm{eV}$ and flow out of the problem at the divertor wall. This high injection temperature was used to keep the upstream temperature at approximately $30 \mathrm{eV}$. The maximum particle velocity is set at $1.5 \times 10^{5} \mathrm{~m} / \mathrm{s}$ which is approximately $3 \times v_{t h}$ for the conditions at the symmetry plane. For this first problem, a constant collision frequency is used. Other important physical quantities are, $v_{t h}=(2 k T / m)^{1 / 2} \approx 5.0 \times 10^{4} \mathrm{~m} / \mathrm{s}$ when $T=30 \mathrm{eV}, f_{n}(L)$ was chosen to give a high recycling solution, and $\nu_{n}=v_{n} / \lambda_{n}$. The rest of the input quantities are defined 
in Table 2.1. Note that $n x$ is the number of cells in the " $x$ " direction and $n v$ is the number of cells in the "v" direction, and Atomic Mass Unit is abbreviated AMU.

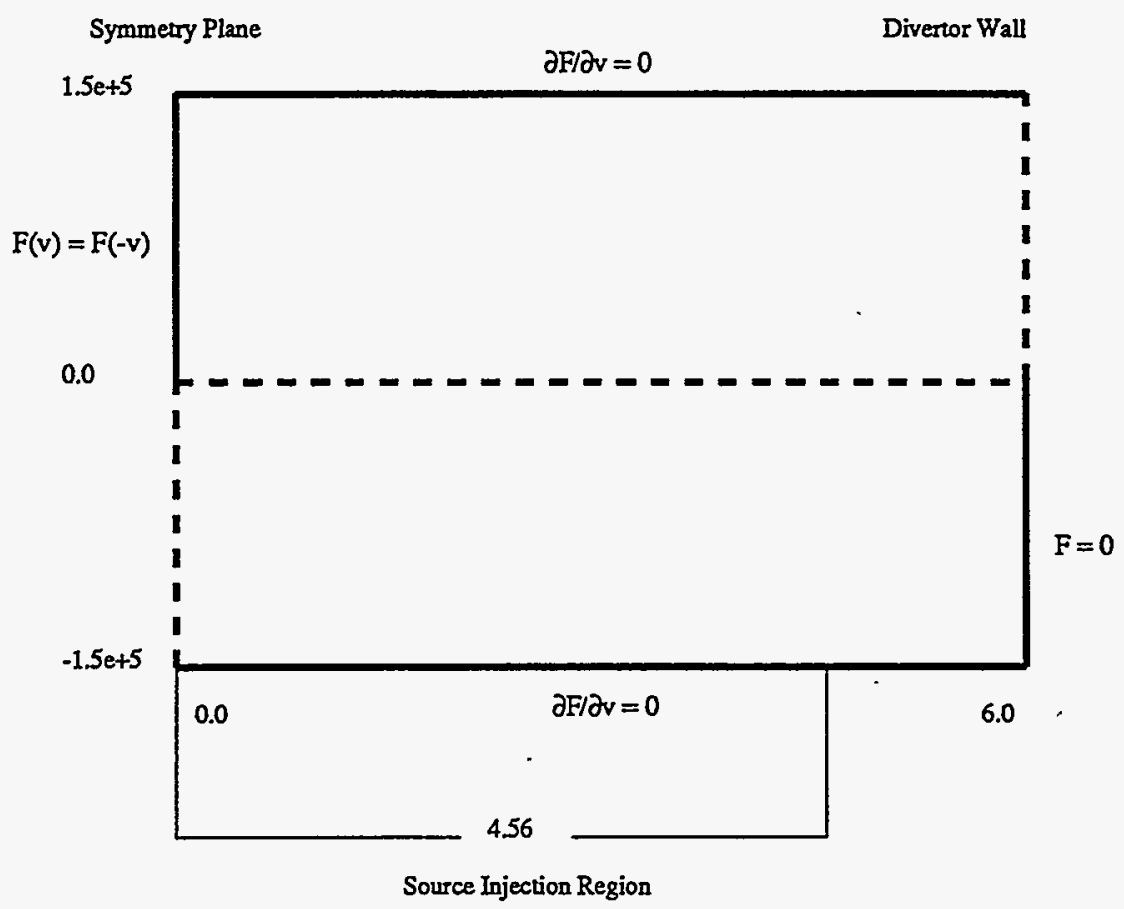

Figure 2.1: Model Problem Geometry

\begin{tabular}{|c|c|c|c|c|c|}
\hline \hline$n x$ & 100 & $n v$ & 200 & $\lambda_{n}$ & $0.5 \mathrm{~m}$ \\
\hline$L$ & $6.0 \mathrm{~m}$ & $v_{\max }$ & $1.5 \times 10^{5} \mathrm{~m} / \mathrm{s}$ & $T_{i n j}$ & $75.0 \mathrm{eV}$ \\
\hline$\nu_{i i}$ & $2.5 \times 10^{5} \mathrm{~s}^{-1}$ & $m$ & $2 \mathrm{AMU} \mathrm{s}$ & $R_{i n j}$ & $3.367 \times 10^{27} \mathrm{~m}^{-3} \mathrm{~s}^{-1}$ \\
\hline$v_{n}$ & $3.5 \times 10^{4} \mathrm{~m} / \mathrm{s}$ & $f_{n}(L)$ & $6.122 \times 10^{19} \mathrm{~m}^{-3}$ & $L_{i n j}$ & $4.56 \mathrm{~m}$ \\
\hline \hline
\end{tabular}

Table 2.1: Input Values

In figure 2.2 one observes the neutral particle density profile which results from equation 2.22. These neutrals are ionized and fed into equation 2.3 through equation 2.23 .

Figure 2.3 shows the electric field computed by equation 2.28 . Here, the increase in the electric field in the pre-sheath region near the wall can be seen.

In figure 2.4 through 2.7 the moment quantities defined by equation 2.4 through 2.7 


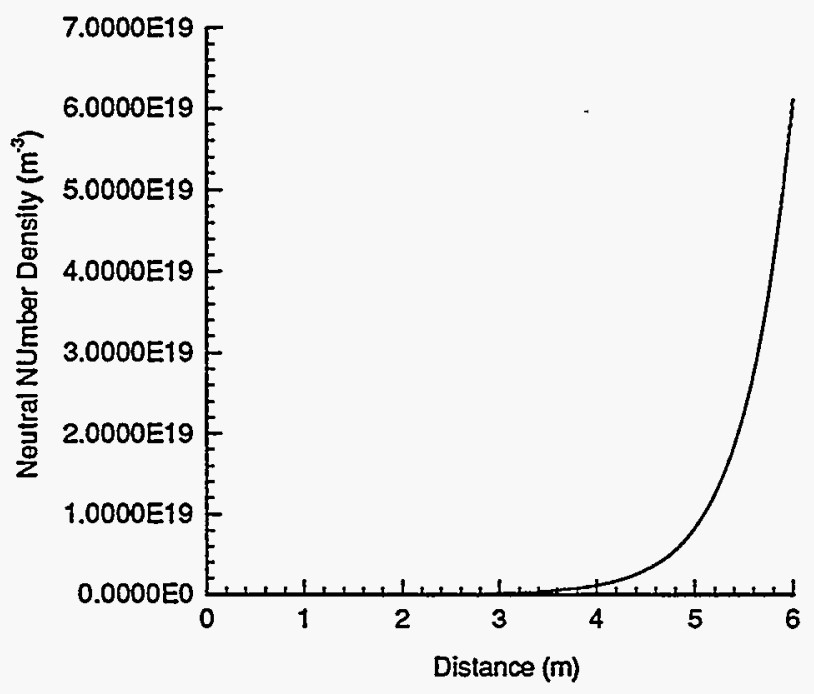

Figure 2.2: Neutral Number Density

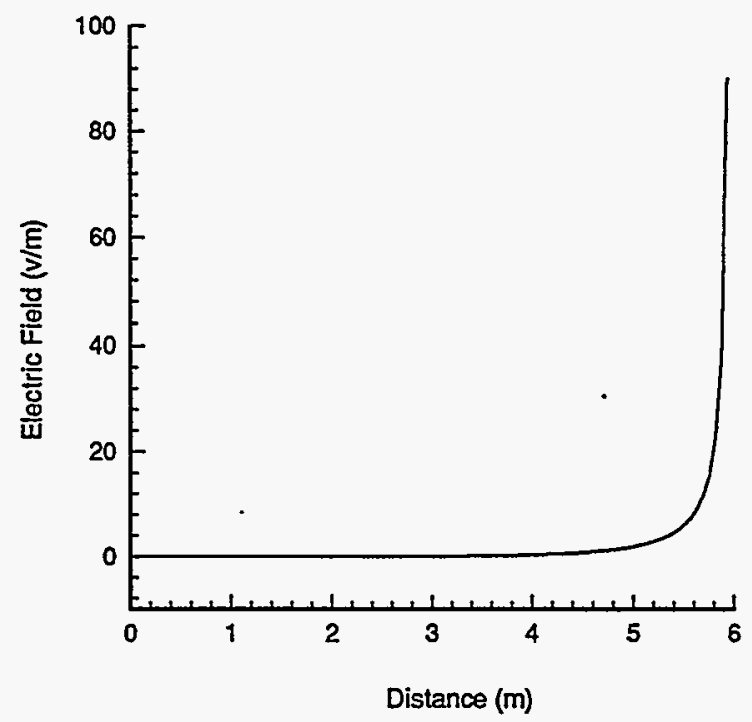

Figure 2.3: Electric Field 


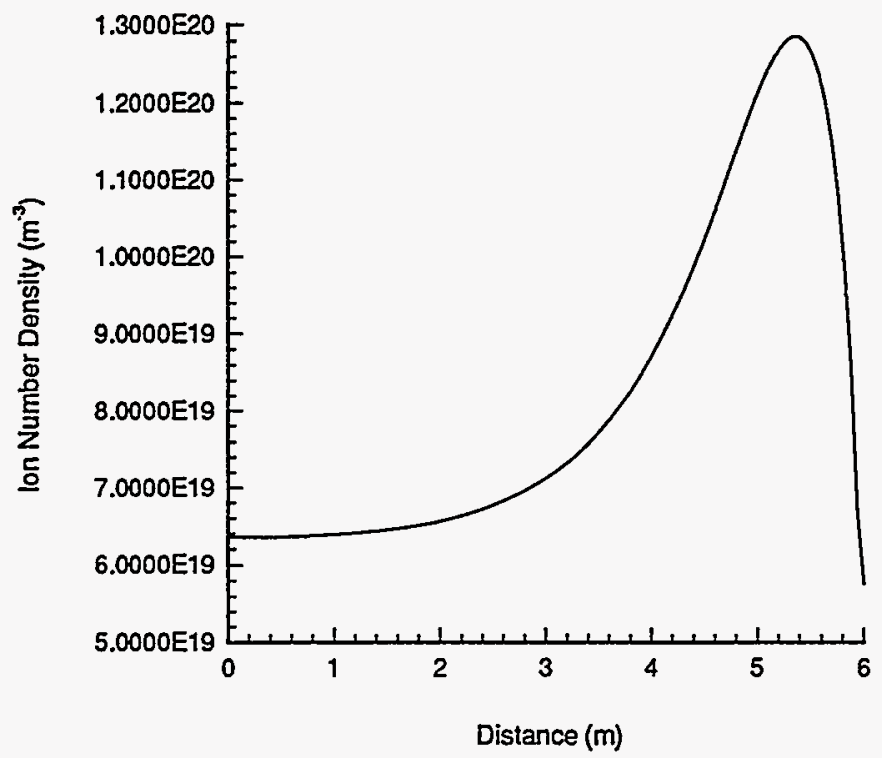

Figure 2.4: Ion Number Density

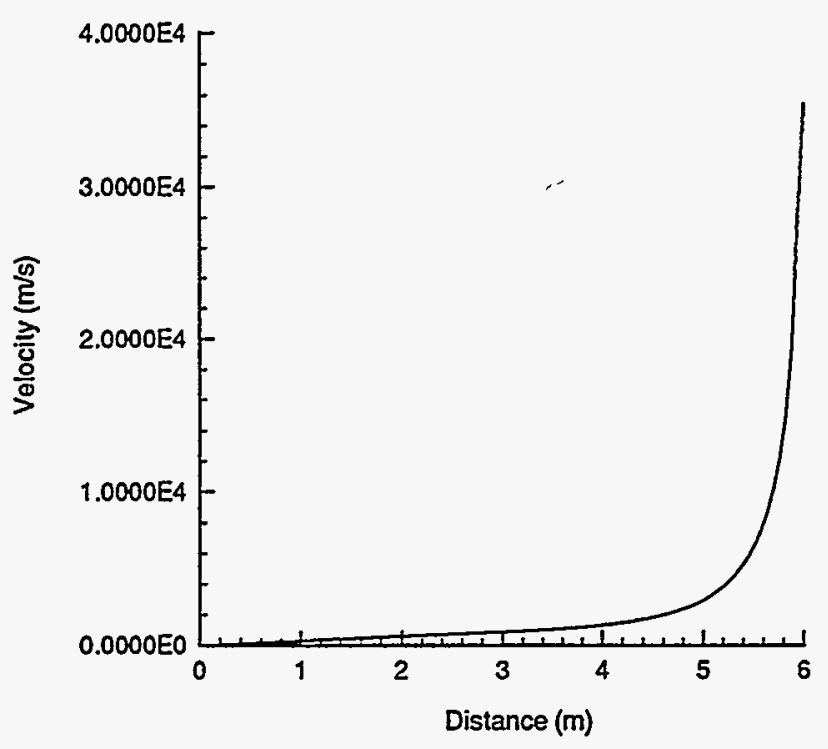

Figure 2.5: Fluid Velocity 


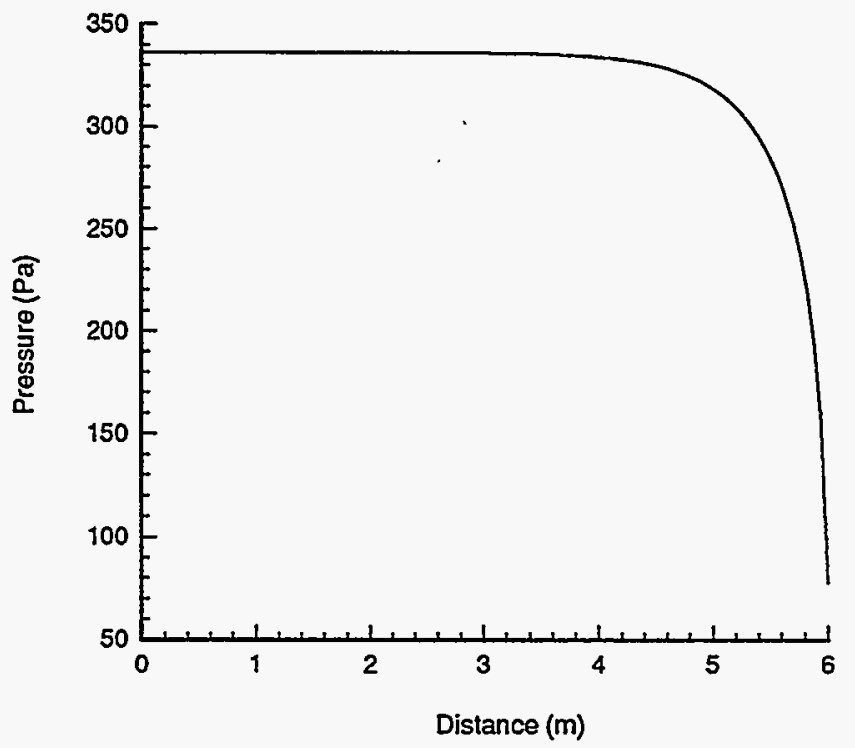

Figure 2.6: Fluid Pressure

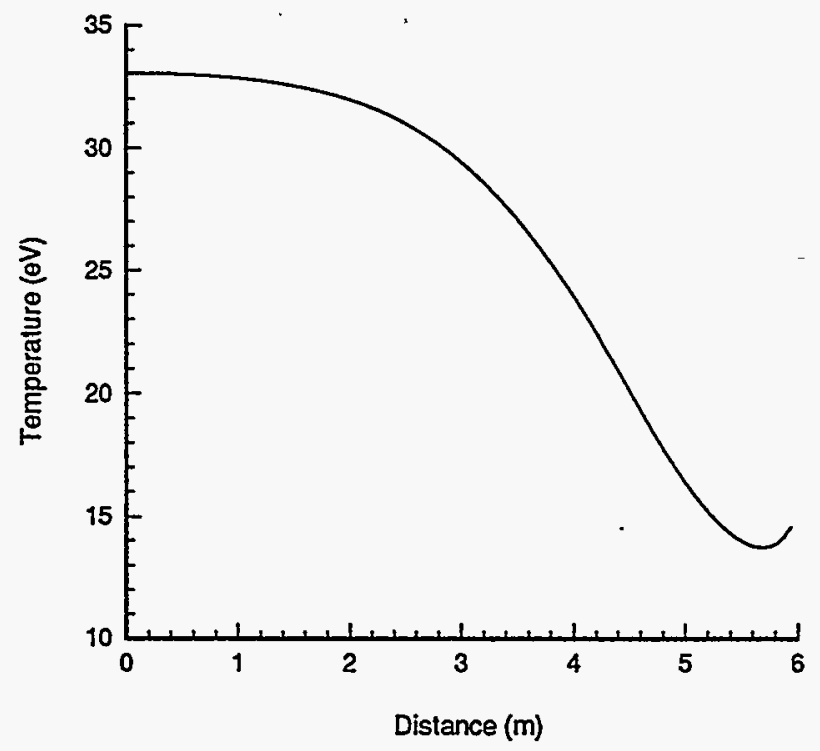

Figure 2.7: Fluid Temperature 
respectively are shown. In these figures, the classic high recycling divertor structure of a density bump and a declining temperature $[31,33]$ is demonstrated.

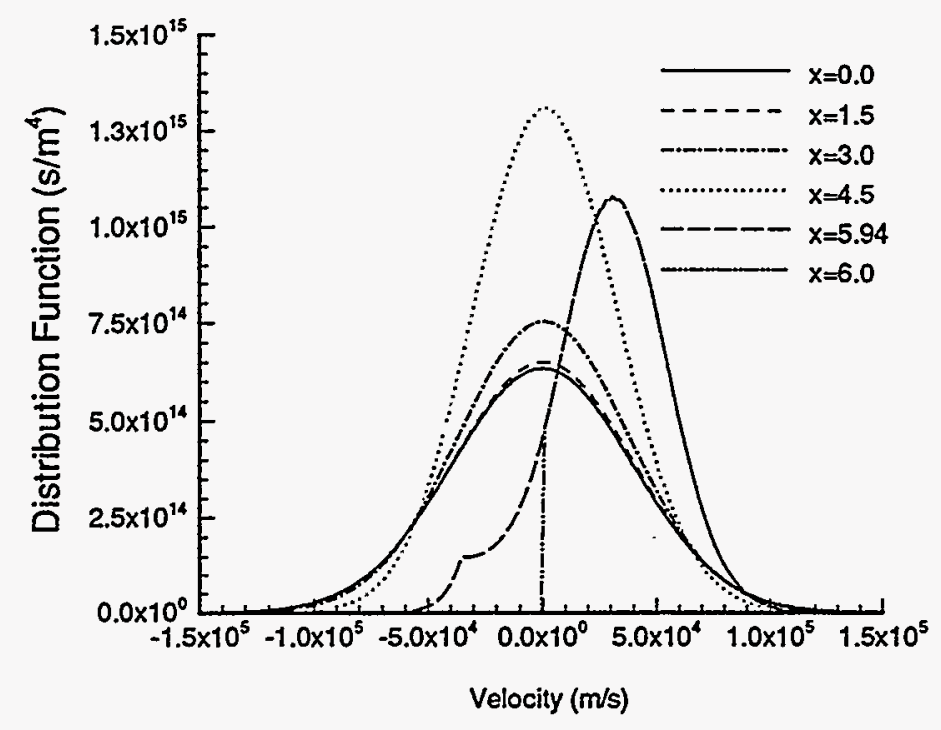

Figure 2.8: Distribution Function Slices

Figure 2.8 shows constant " $x$ " slices of the distribution function. Here, the acceleration of the flow as it moves across the simulation towards the divertor is shown by the shifting of the distribution function towards higher velocity. The spatial dependence of the number density is also revealed. The plot shows the initial rise in number density, peak at $x=4.5$, and the the drop as the wall is approached, $x=6.0$. Figure 2.8 also demonstrates the non-Maxwellian behavior caused by the ionization source at $x=5.94$. The ionization source is shown as a bump at $v_{n}=-3.5 \times 10^{4} \mathrm{~m} / \mathrm{s}$.

Figure 2.9 shows the convergence, in terms of update size $\delta y^{n}$ in equation 2.34 , per Newton step for each of the four meshes. Here it is clearly shown that mesh sequencing reduces the 


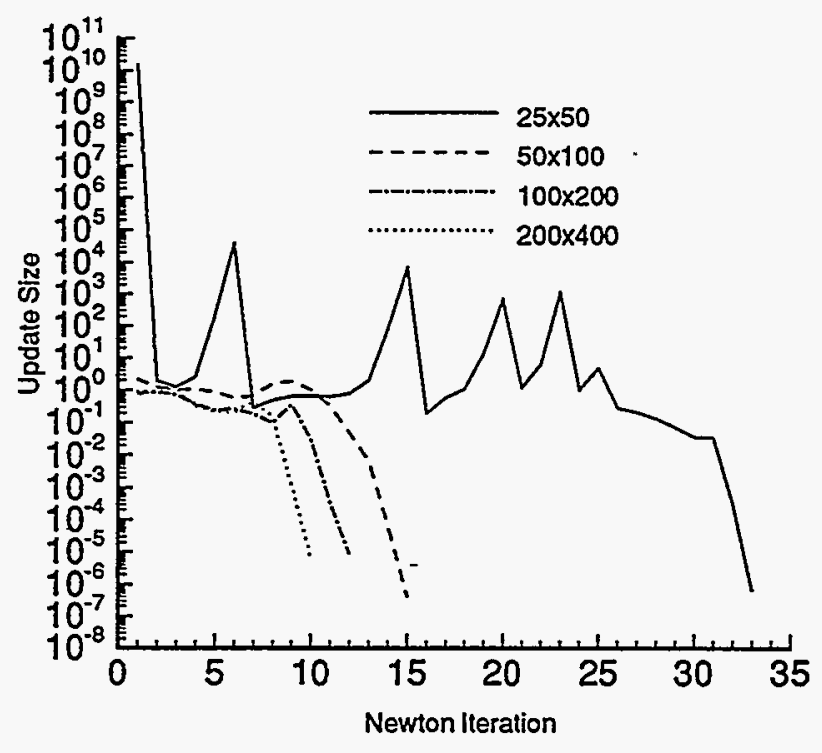

Figure 2.9: Mesh Sequenced Convergence vs. Newton Iteration

number of Newton iterations on the finer meshes. The strong oscillations in the coarse grid convergence are a function of the pseudo-transient progression, the damping algorithm, and the adjustable time step.

\begin{tabular}{|c|c|c|c|}
\hline \hline MESH & NEWTON ITERATIONS & CPU TIME (s) & CUMULATIVE CPU TIME (s) \\
\hline \hline $25 \times 50$ & 33 & 24 & 24 \\
\hline $50 \times 100$ & 15 & 66 & 90 \\
\hline $100 \times 200$ & 12 & 305 & 395 \\
\hline $200 \times 400$ & 10 & 1682 & 2077 \\
\hline \hline
\end{tabular}

Table 2.2: Mesh Sequencing

Figure 2.10 shows the convergence for the $200 \times 400$ mesh as a function of CPU time. Here, the CPU time is for an HP 735 workstation. This illustrates the importance of a good initial guess for the fine grids, where each Newton iteration takes significantly more time than the 


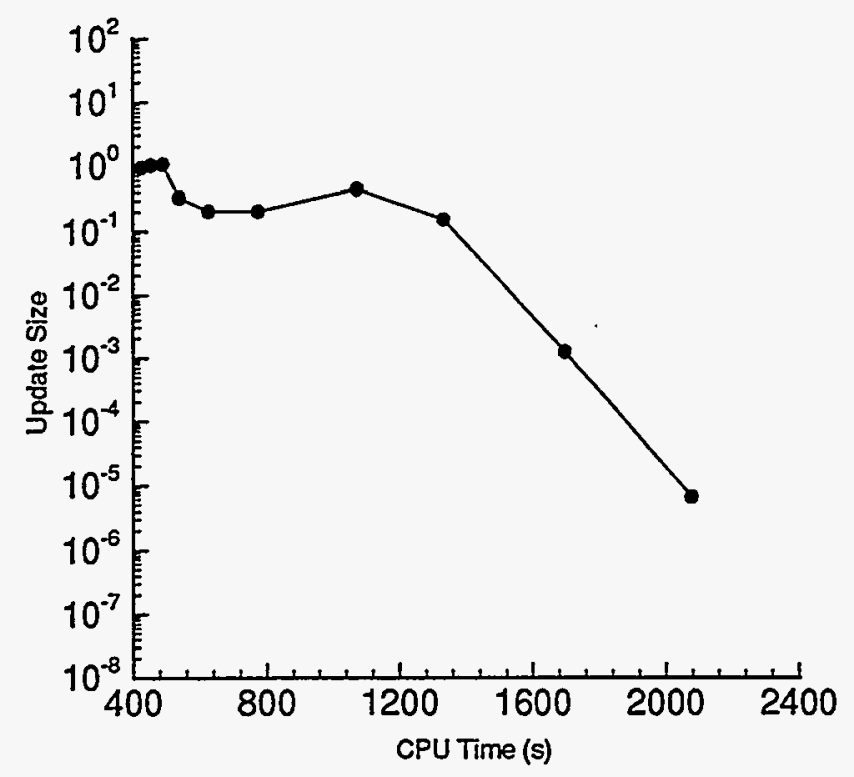

Figure 2.10: 200x400 Convergence vs. CPU Time

coarse grid iterations. Table 2.2 shows the Newton iterations and CPU time for the entire mesh-sequenced run. From figure 2.10, one can see that as the solution is approached, the CPU cost per Newton iteration goes up, since the horizontal spacing of points is increased. This is caused by the Krylov convergence tolerance from equation 2.36 being tightened as the residual gets smaller, therefore, there are more Krylov iterations required for the latter Newton steps.

Figure 2.11 shows the effect of mesh refinement on the fluid pressure. It is clear that the solution is beginning to converge, but it may take many more doublings to get the solution fully converged. These results indicate the need for higher order differencing of the first order operators which are currently upwind differenced (see Section 4.2) everywhere except in the $\left(\frac{\partial F}{\partial t}\right)_{c}$ term where interpolated donor cell differencing (see Section 4.3.1) is used. Higher 


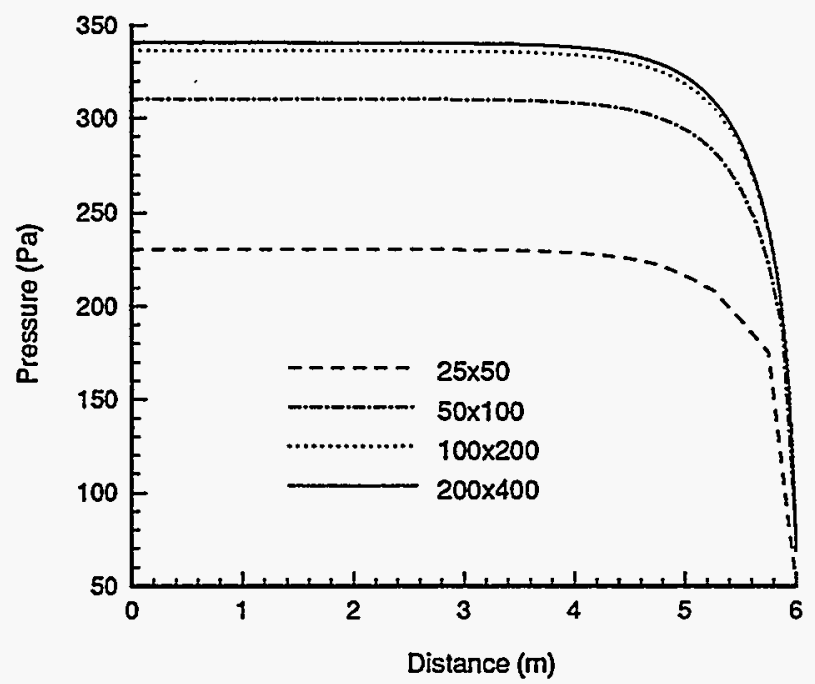

Figure 2.11: Mesh Sequenced Pressure

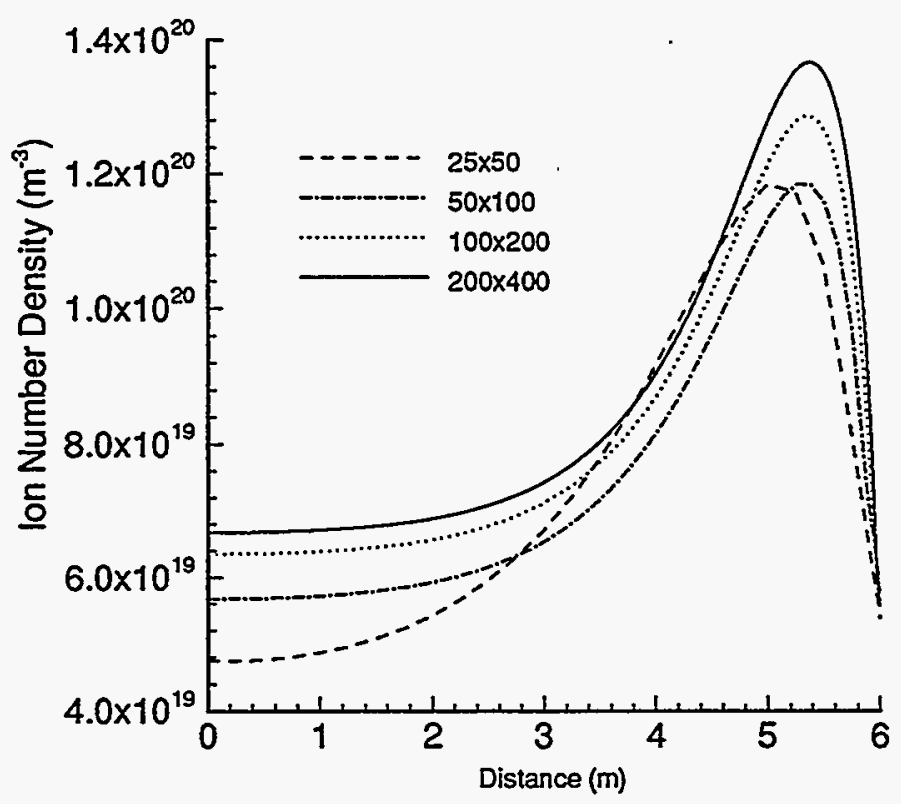

Figure 2.12: Mesh Sequenced Ion Density 
order differencing as well as a non-uniform grid will aid in limiting the required mesh size for accuracy. In figure 2.12 one can more clearly see that the solution has not yet reached a mesh independent solution. For a further discussion of this and mesh converged solutions, see Section 4.5 in Chapter 4.

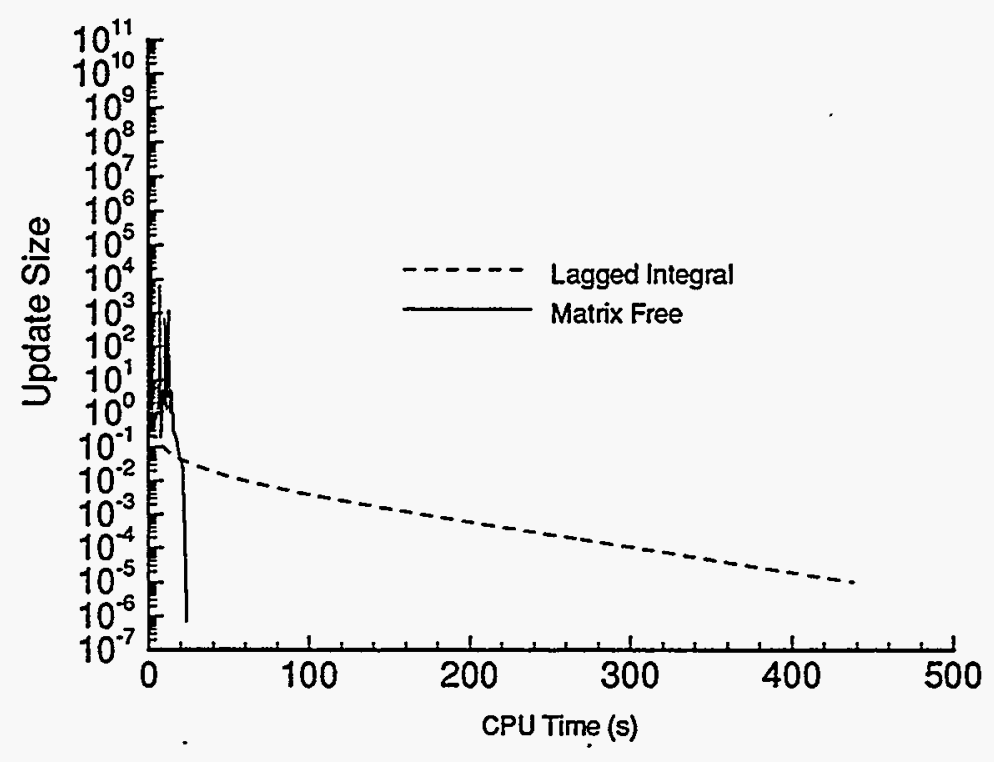

Figure 2.13: Matrix Free vs. Lagged Integral CPU Time

Figure 2.13 and figure 2.14 show the importance of keeping the integral quantities at new time for convergence. In the matrix free option, all integral quantities are at new time and it takes only 33 iterations to converge, compared to 1975 if the integral quantities are lagged to the old iteration value. Although it costs more in terms of CPU time per iteration, one can clearly see from figure 2.13 that the matrix free option is more computationally efficient by an order of magnitude.

Figure 2.15 and figure 2.16 show the effect of the neutral mean free path on the fluid 


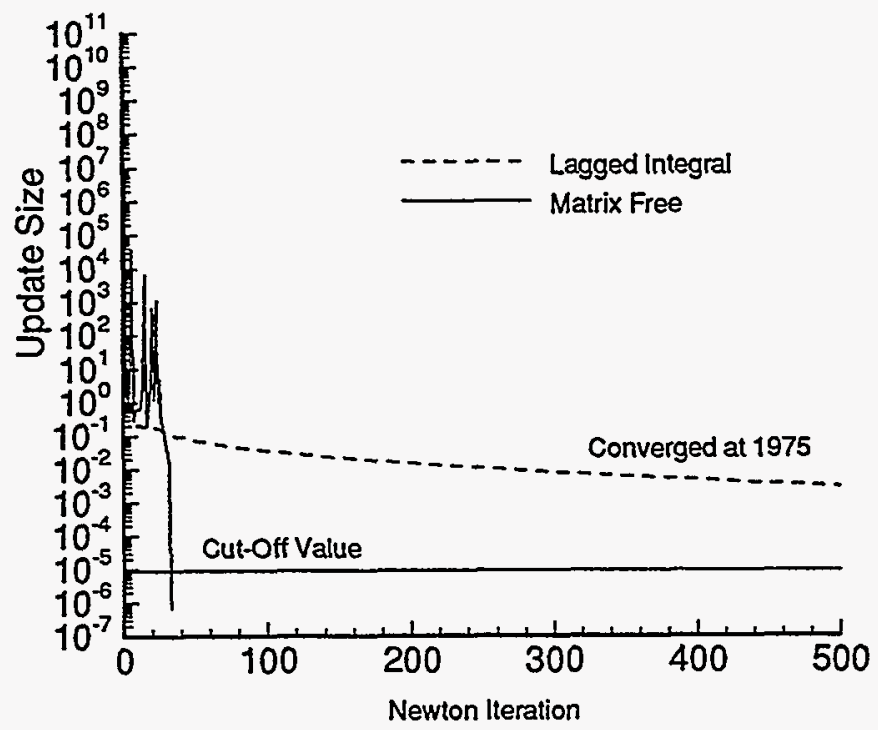

Figure 2.14: Matrix Free vs. Lagged Integral Newton Iterations

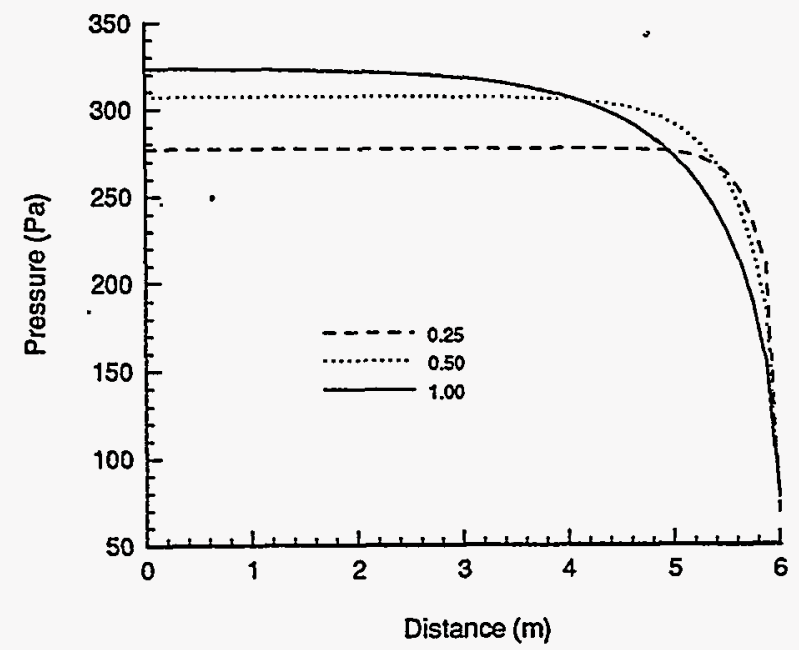

Figure 2.15: Fluid Pressure vs. Neutral MFP 


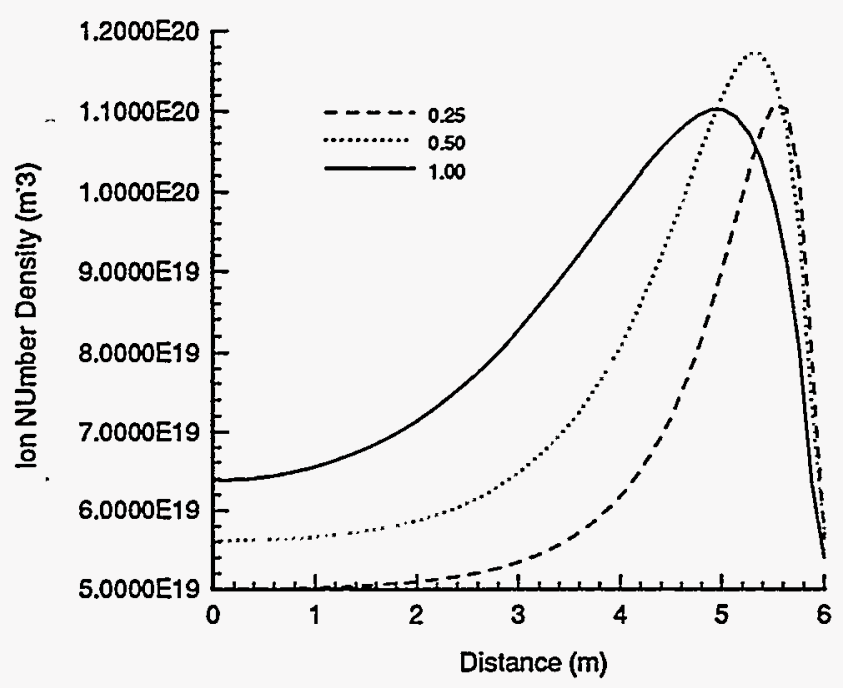

Figure 2.16: Ion Number Density vs. Neutral MFP

pressure and the ion number density. Here the importance of the neutral diffusion model is clearly demonstrated by the wide shifts in the size and the location of the ion number density peak. These plots show the same simulation for three different choices of $\lambda_{n},(1 \mathrm{~m}, 1 / 2 \mathrm{~m}$, and $1 / 4 \mathrm{~m}$ ). From figure 2.16 it is easy to see that as $\lambda_{n}$ is made smaller, the neutrals are ionized much closer to the plate. These large differences indicate the importance of including a more realistic neutral particle model.

For the earlier results, a constant collision frequency was used in equation 2.8. Next, the non-linearity and integral coupling will be increased by making the collision frequency a function of $x, T$, and $n$. Figure 2.17 and figure 2.18 show both the matrix-free and the lagged integral solutions for the $x$ dependent collision frequency $\left(\nu_{i i}=K \frac{n}{T^{3 / 2}}\right)$. Again, the matrix-free solution requires significantly less time and fewer Newton iterations to obtain the 


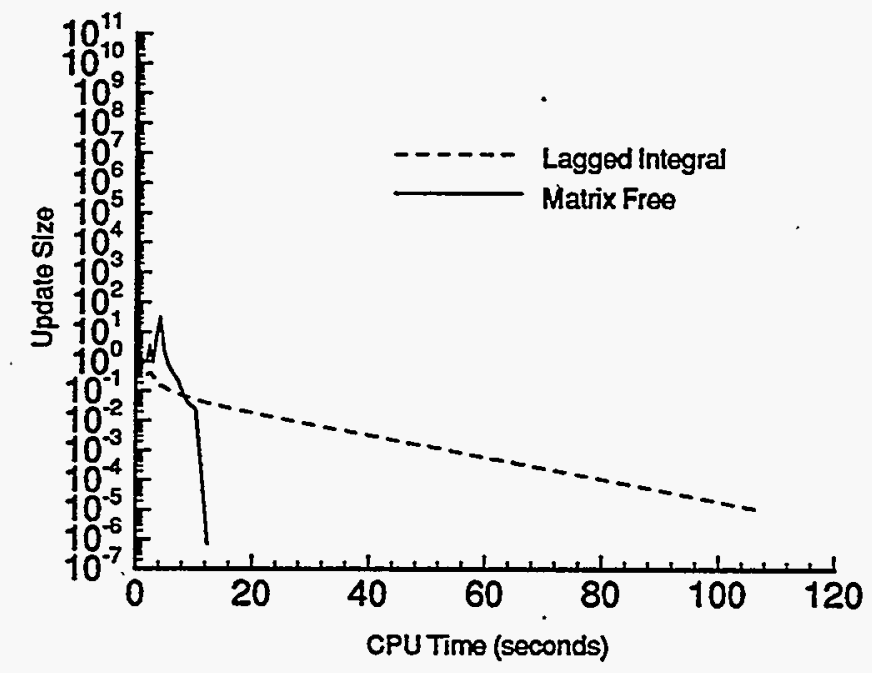

Figure 2.17: Matrix Free vs. Lagged Integral CPU Time for Variable Collision Frequencies

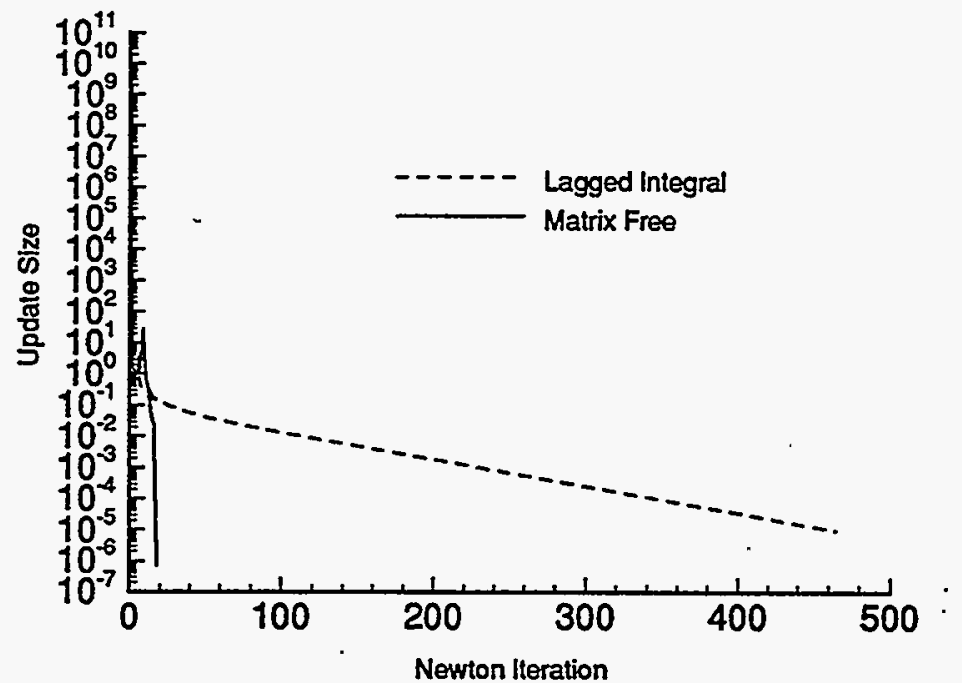

Figure 2.18: Matrix Free vs. Lagged Integral Newton Iteration for Variable Collision Frequencies 


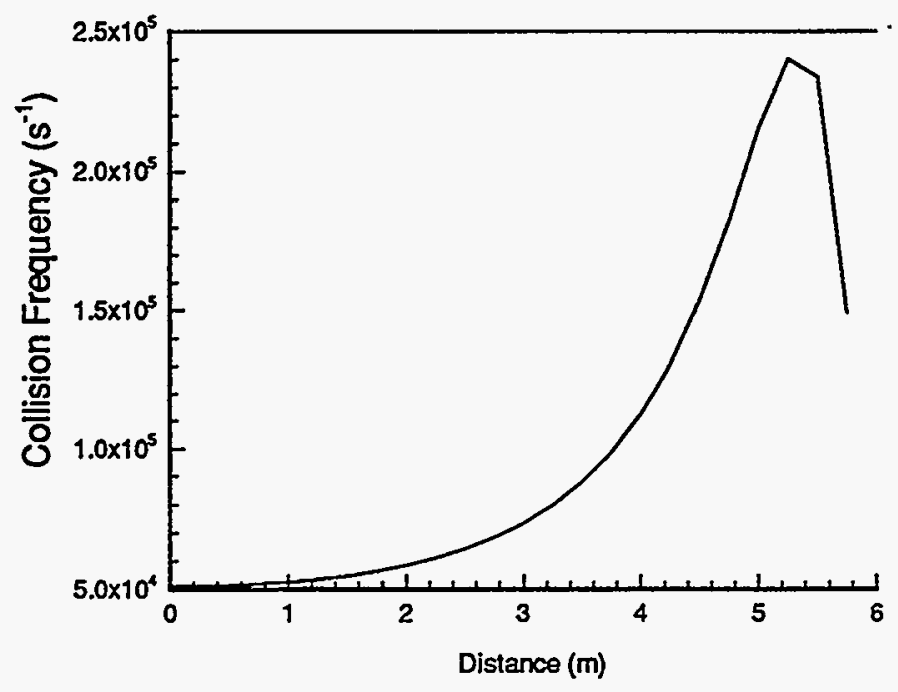

Figure 2.19: Collision Frequency vs. Distance

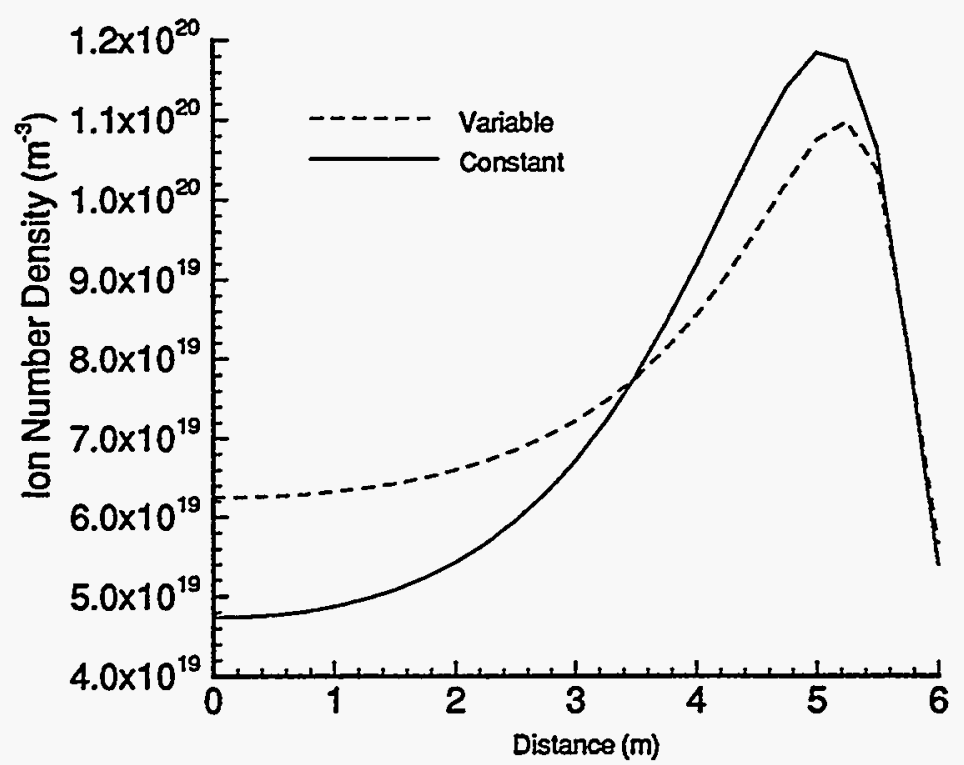

Figure 2.20: Ion Number Density Constant vs. Variable Collision Frequency 


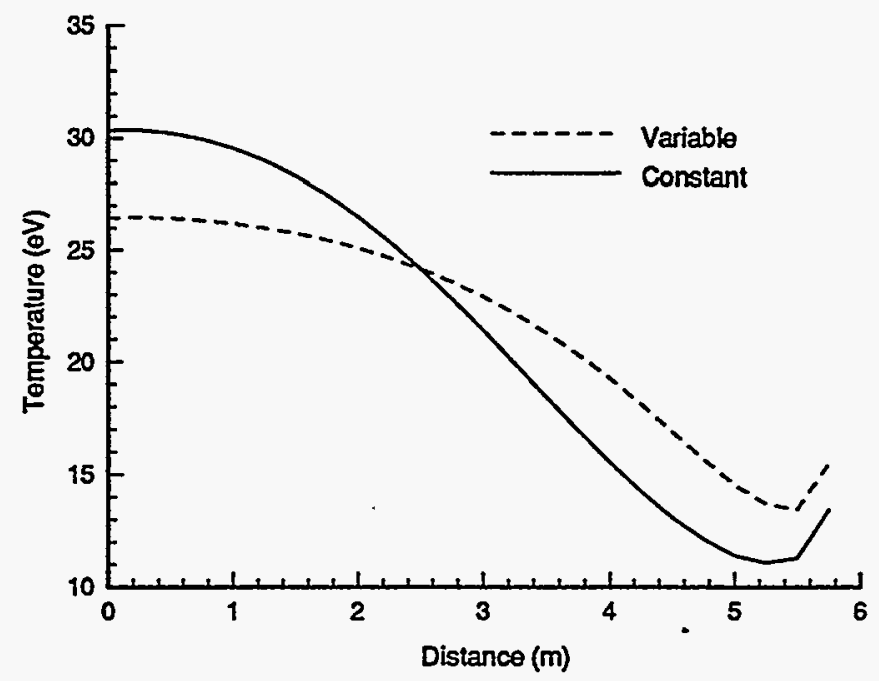

Figure 2.21: Fluid Temperature Constant vs. Variable Collision Frequency

same steady state solution. It should be noted also from figure 2.19 , which shows the $\frac{n}{x^{\frac{3}{2}}}$ dependency of the ion-ion collision frequency, that the collision frequency is lower for the $x$-dependent $\nu_{i i}$. To get a more accurate collision operator, one would also have to include the particle velocity dependence. Figure 2.20 and figure 2.21 illustrate the effect of variable collision frequency on ion number density and fluid temperature, respectively. These plots illustrate that the lower variable collision frequency results in flatter profiles. This indicates that the collision frequency needs to be high to support steep gradients.

Figure 2.15 and figure 2.16 showed how changing $\lambda_{n}$ in equation 2.23 effected the solution. In equation 2.23 the neutral particles were assumed to be a beam of particles (i.e., $T_{n}=0$ ) all moving at velocity $v_{n}$. For an alterative model the neutral particles have a Maxwellian distribution at a given neutral particle temperature. The new form of the neutral particle 


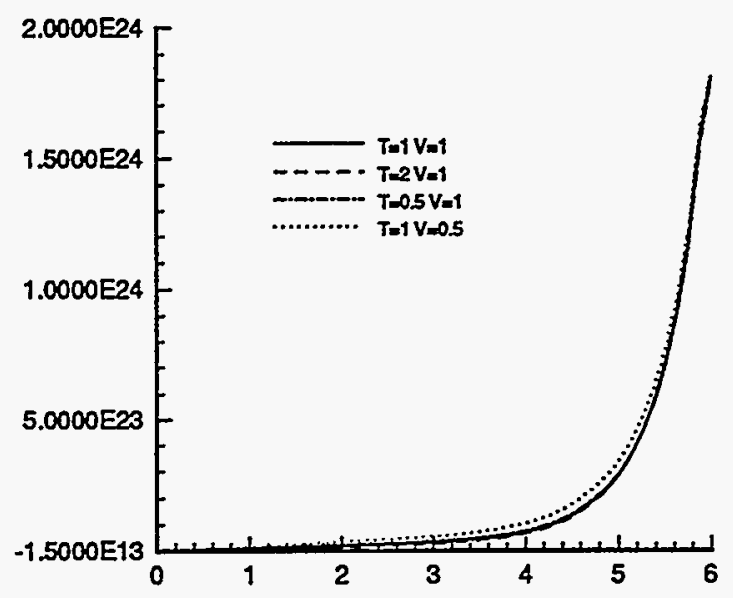

Figure 2.22: Particle Flux vs. Injection Shape

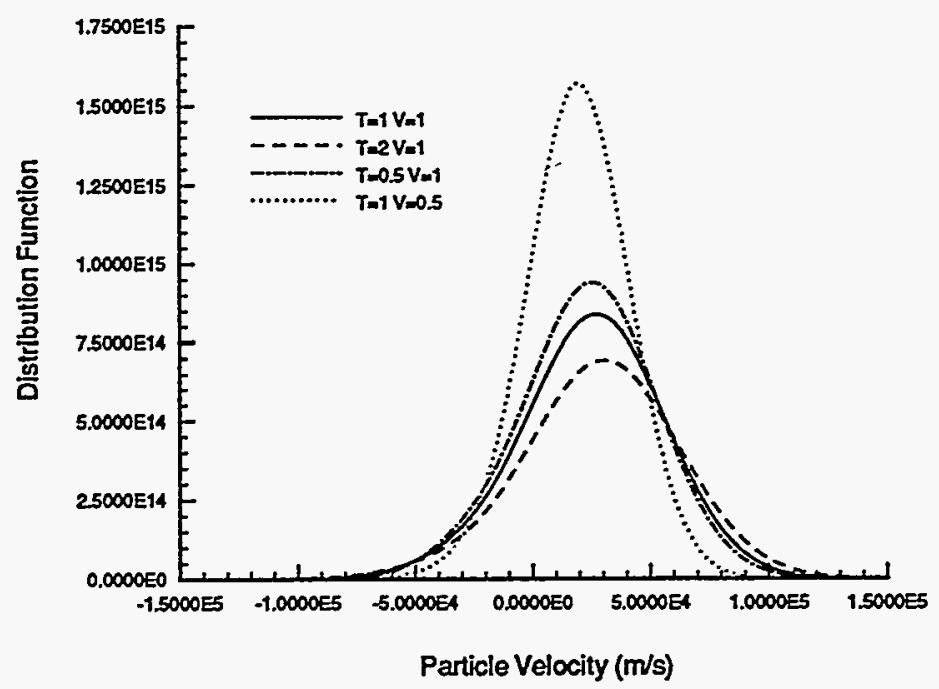

Figure 2.23: Distribution Function vs. Injection Shape 


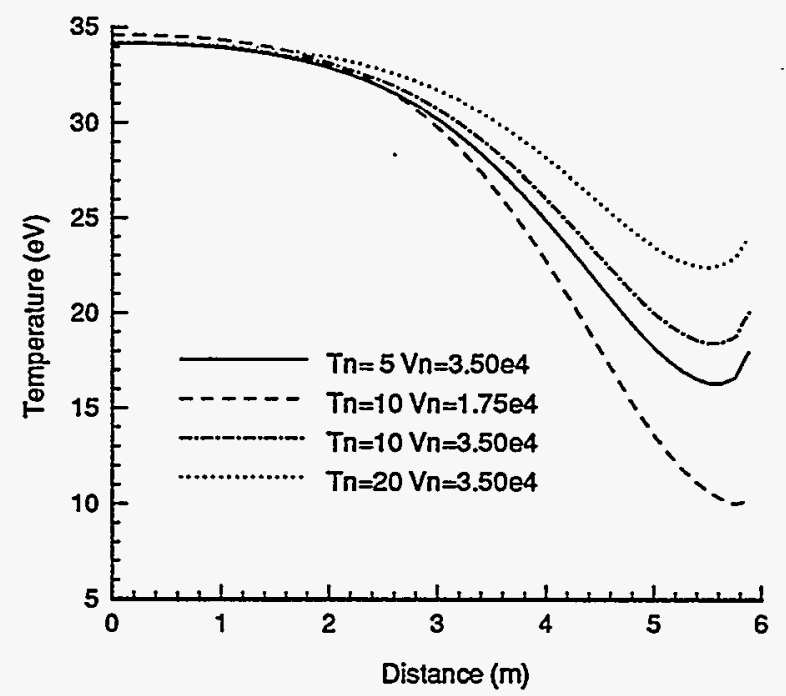

Figure 2.24: Fluid Temperature vs. Injection Shape

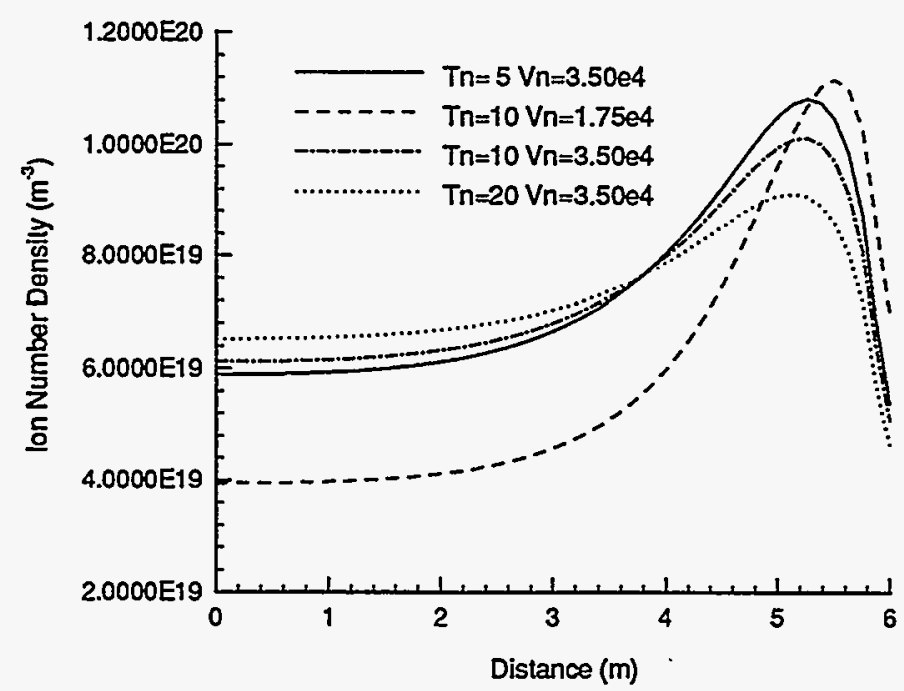

Figure 2.25: Particle Density vs. Injection Shape 
ionization term is:

$$
\left(\frac{\partial F}{\partial t}\right)_{i n z}=f_{n 0}(x) \exp \left[\frac{-\left(v-v_{n}\right)^{2}}{v_{n t h}^{2}}\right]
$$

where

$$
\begin{gathered}
v_{n t h}=\left(\frac{2 k T_{n}}{m_{n}}\right)^{1 / 2} \\
f_{n 0}(x)=f_{n}(x)\left(\frac{1}{v_{n t h}^{2} \pi}\right)^{1 / 2},
\end{gathered}
$$

and $f_{n}(x)$ is defined as before in equation 2.22. It is assumed that $m_{i}=m_{n}$, or that the mass of the electron relative to the ion is negligible. Figure 2.22 through figure 2.25 demonstrate the effect of the Maxwellian neutral distribution function on the divertor simulation. The different lines on the plots indicate different choices for $T_{n}$ and $v_{n}$ in this neutral particle model. Figure 2.22 serves as a check to show that the models are consistent and that the neutral particle ionization rate is the same for all the different neutral particle distribution function shapes. Figure 2.23 shows the distribution functions near the plate. Now the spike from the neutral beam which was apparent in figure 2.8 has been smoothed out. Figure 2.24 demonstrates how the neutral particle model effects the cooling of the ions as they approach the plate. In figure 2.25 the densities near the plate are clearly changed by the new neutral model. One can see that assumptions about the shape of the neutral particle distribution function can very strongly effect the simulation near the plate, again indicating the importance of an accurate neutral particle transport model.

Figure 2.26 shows the effect of the level of $\Pi U$ fill-in [58] on the average number of Krylov iterations per Newton iteration. The simulation uses initial conditions which are the steady state solution to the base problem, see Table 2.1, where $\nu_{i i}$ in equation 2.8 was perturbed from $2.5 \times 10^{5}$ to $2.4 \times 10^{5}$ to initiate the transient. This is the transient used to generate 


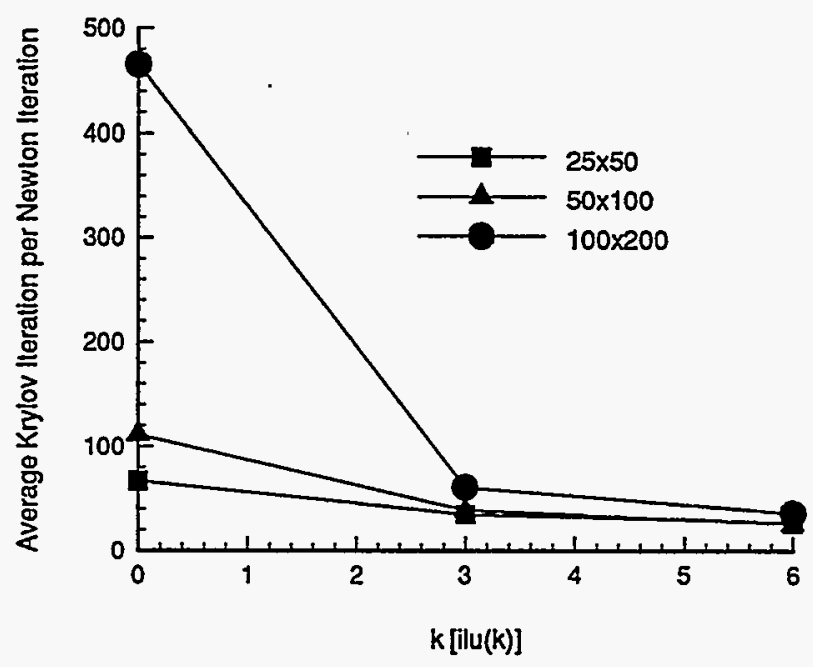

Figure 2.26: Average Krylov Iteration vs. Level of ILU Fill-In

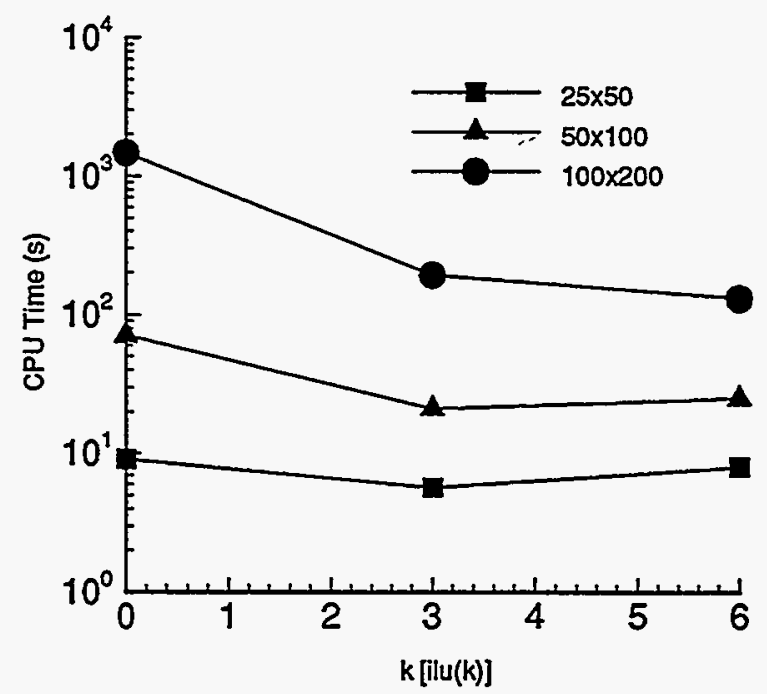

Figure 2.27: Run Time vs. Level of ILU Fill in for Different Grids 
figure 2.26 through figure 2.29 . The plot clearly shows the break-down of ILU(0) as a function of problem size, (the upper limit on Krylov iterations is set at 480 and the $200 \times 400$ ILU(0) problem hit this limit two of the four Newton iterations). However, it also demonstrates that higher levels of $I L U$ fill-in, although requiring much more memory and CPU time, scale better with the problem size than $I L U(0)$. Figure 2.27 shows the effect of level of $I U U$ fill-in on the run time (note that the CPU time is on a logarithmic scale). Here, the most powerful preconditioner ILU(6), is also the most effective for the larger grids. This is a result of the high cost per Krylov iteration on the large grids caused by the matrix-free approximation.

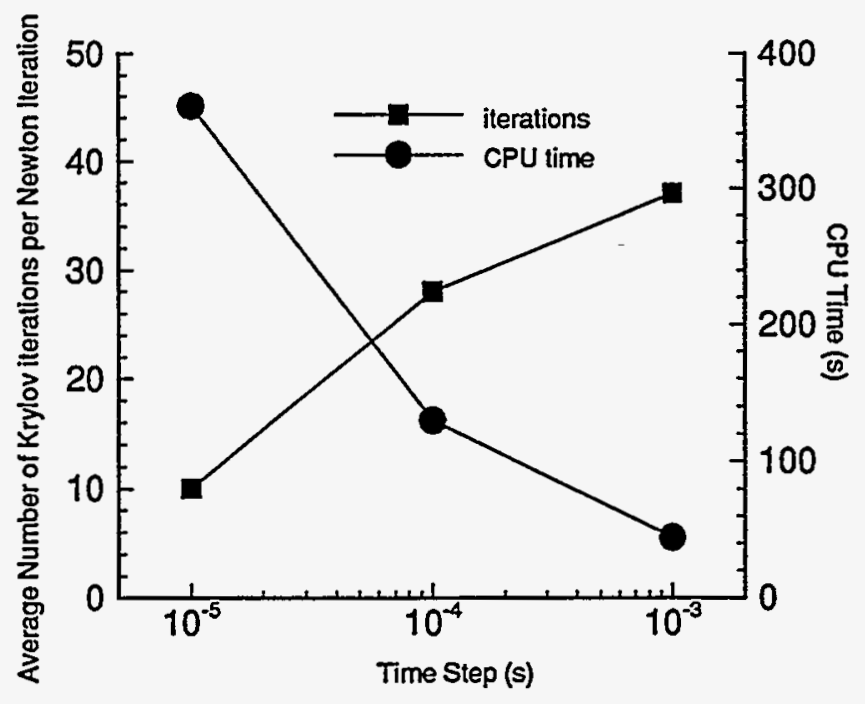

Figure 2.28: Average Krylov Iteration and Run Time vs. Time Steps Size

The effect of time step size, $\Delta t$ from equation 2.37 , on the Krylov iteration and the run time is presented in figure 2.28 showing that as the time step size increases the CPU time decreases, even though the number of iterations per Newton step increases. Recall that since 
this is a pseudo-transient, and not a "real" transient, it does not take 100 times as many steps to reach steady state for $\Delta t=1.0 \times 10^{-5}$ as $\Delta t=1.0 \times 10^{-3}$ (the actual numbers are 7 and 152 , or a ratio of about 22 ).

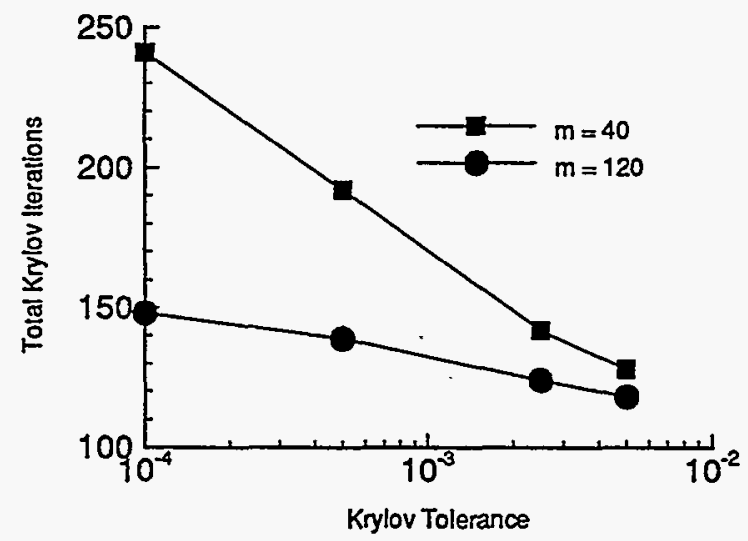

Figure 2.29: Total Krylov Iteration vs. Krylov Tolerance for Different Maximum Krylov Space Dimensions

The effect of the Krylov convergence tolerance, tolcg from equation 2.36, on the number of Krylov iterations is presented in figure 2.29 for GMRES(40) and GMRES(120), where the maximum dimension of the Krylov space are 40 and 120 , respectively. Since the GMRES algorithm's memory usage increases with the number of iterations, an upper bound is set (i.e., 40 or 120). If the number of iterations exceeds the preset limit, the algorithm is restarted. In figure 2.29 the effect of restarting the GMRES [59] algorithm is clearly demonstrated by the large increase in the total number of Krylov iterations from GMRES(120) to GMRES(40). With the maximum Krylov dimension set to 120 , there are no restarts of the GMRES algorithm. When the maximum is set to 40 , the algorithm has to restart often. 
When the GMRES algorithm is restarted the convergence rate is slowed and sometimes stalls as discussed in references $[32,53,54]$.

\subsection{Summary}

In this chapter it has been shown that the Matrix-Free Newton Krylov method is an effective technique for solving the Vlasov Fokker Planck equation. This technique solves the integro-differential equations in a fully coupled manner. The method does not require extensive memory since the matrix is approximated by function evaluations. It has also been demonstrated that the fully coupled method converges much faster than lagging the integral terms one iteration level behind. 


\section{Chapter 3}

\section{One Velocity (0D1V) Ion and}

\section{Electron}

\subsection{Introduction}

Now that the solution algorithm has been described and demonstrated on the 1D1V ion problem, the next step towards the goal of solving the $1 \mathrm{D} 1 \mathrm{~V}$ problem with coupled ions and electrons will be taken. For this chapter, the spatial dimension is dropped and concentration is focused on the collision operators. First, the zero-spatial dimension electron Vlasov-FokkerPlanck equation is added. Next, ion-electron collisions are be added in both the ion and electron kinetic equations. These collision operators, which have the same form as the ion-ion and electron-electron simplified operators, account for the momentum and energy exchange between the ions and electrons.

The rest of this chapter has the following organization. Section 3.2 presents the OD1V ion-electron equations and describes the new collision operators. Section 3.3 describes the 
effect of the new equation and the new collision operators on the structure of the matrix. Section 3.4 discusses some of the difficulties associated with differencing the simplified FokkerPlank collision operator and shows how the differencing was improved. Section 3.5 presents the model problems used to test the implementation of the unlike particle collision operators, derives their exact solution, and compares the code results to the exact solution. Section 3.6 summarizes the chapter.

\subsection{Mathematical Model}

The ion equation for the OD1V work is very similar to the 1D1V ion VFP equation (equation. 2.3), but the convective term, $v \frac{\partial F}{\partial x}$, is neglected and there are now two collision operators. Also for the 0D1V work there is no source terms (i.e., $\left.\left(\frac{\partial F}{\partial t}\right)_{i n z}=\left(\frac{\partial F}{\partial t}\right)_{s r c}=0\right)$. With these assumptions the OD1V ion equation becomes,

$$
\frac{\partial F_{i}}{\partial t}+\frac{q_{i} E}{m_{i}} \frac{\partial F_{i}}{\partial v_{i}}=\left(\frac{\partial F_{i}}{\partial t}\right)_{i i}+\left(\frac{\partial F_{i}}{\partial t}\right)_{i e}
$$

The electron equation with the same assumptions is given by:

$$
\frac{\partial F_{e}}{\partial t}+\frac{q_{e} E}{m_{e}} \frac{\partial F_{e}}{\partial v_{e}}=\left(\frac{\partial F_{e}}{\partial t}\right)_{e e}+\left(\frac{\partial F_{e}}{\partial t}\right)_{e i}
$$

The distribution function moments are defined as follows:

$$
\begin{aligned}
n_{i} & =\int_{-\infty}^{\infty} F_{i} d v_{i} \\
n_{i} \bar{V}_{i} & =\int_{-\infty}^{\infty} v_{i} F_{i} d v_{i} \\
n_{i} k T_{i} & =m_{i} \int_{-\infty}^{\infty}\left(v_{i}-\bar{V}_{i}\right)^{2} F_{i} d v_{i}
\end{aligned}
$$




$$
\begin{aligned}
n_{e} & =\int_{-\infty}^{\infty} F_{e} d v_{e} \\
n_{e} \bar{V}_{e} & =\int_{-\infty}^{\infty} v_{e} F_{e} d v_{e} \\
n_{e} k T_{e} & =m_{e} \int_{-\infty}^{\infty}\left(v_{e}-\bar{V}_{e}\right)^{2} F_{e} d v_{e}
\end{aligned}
$$

The like-particle collision operators have the same form as before,

$$
\begin{gathered}
\left(\frac{\partial F_{i}}{\partial t}\right)_{i i}=\nu_{i i}\left\{\left[\frac{k T_{i}}{m_{i}}\right] \frac{\partial^{2} F_{i}}{\partial v_{i}^{2}}+\frac{\partial\left[\left(v_{i}-\bar{V}_{i}\right) F_{i}\right]}{\partial v_{i}}\right\} \\
\left(\frac{\partial F_{e}}{\partial t}\right)_{e e}=\nu_{e e}\left\{\left[\frac{k T_{e}}{m_{e}}\right] \frac{\partial^{2} F_{e}}{\partial v_{e}^{2}}+\frac{\partial\left[\left(v_{e}-\bar{V}_{e}\right) F_{e}\right]}{\partial v_{e}}\right\},
\end{gathered}
$$

and the unlike collision operators from Catto [8] are,

$$
\begin{gathered}
\left(\frac{\partial F_{i}}{\partial t}\right)_{i e}=\nu_{i e}\left\{\left[\frac{k T_{e}}{m_{i}}\right] \frac{\partial^{2} F_{i}}{\partial v_{i}^{2}}+\frac{\partial\left[\left(v_{i}-\bar{V}_{e}\right) F_{i}\right]}{\partial v_{i}}\right\} \\
\left(\frac{\partial F_{e}}{\partial t}\right)_{e i}=\nu_{e i}\left\{\left[\frac{k T_{e}}{m_{e}}+\varepsilon\right] \frac{\partial^{2} F_{e}}{\partial v_{e}^{2}}+\frac{\partial\left[\left(v_{e}-\bar{V}_{i}\right) F_{e}\right]}{\partial v_{e}}\right\},
\end{gathered}
$$

where,

$$
\varepsilon=K\left(\frac{T_{i}-T_{e}}{m_{i}}\right)+\left(\bar{V}_{e}-\bar{V}_{i}\right)^{2}
$$

and $\varepsilon$ is a thermal equilibrium term which must be included to conserve momentum and energy in the electron-ion collision operator. Where Catto [8] gives the collision frequencies the following form,

$$
\begin{gathered}
\nu_{i i}=\frac{4 \pi^{1 / 2} e^{4} n_{i} \ln \Lambda}{3 m_{i}^{1 / 2}\left(k T_{i}\right)^{3 / 2}} \\
\nu_{e i}=\frac{4(2 \pi)^{1 / 2} e^{4} n_{i} \ln \Lambda}{3 m_{e}^{1 / 2}\left(k T_{e}\right)^{3 / 2}}
\end{gathered}
$$




$$
\begin{gathered}
\nu_{i e}=\frac{m_{e} n_{e}}{m_{i} n_{i}} \nu_{e i} \\
\nu_{e e}=\frac{n_{e}}{n_{i}} \nu_{e i} .
\end{gathered}
$$

Here,

$$
\Lambda=12 \pi n_{e} \lambda_{D}^{3}
$$

where,

$$
\lambda_{D}=\left(\frac{\epsilon_{0} k T_{e}}{n_{e} e^{2}}\right)^{1 / 2}
$$

is the Debye length and $e$ is the charge of an electron and $\epsilon_{0}$ is the permittivity of free space.

\subsection{New Matrix Structure}

IONS

Electrons

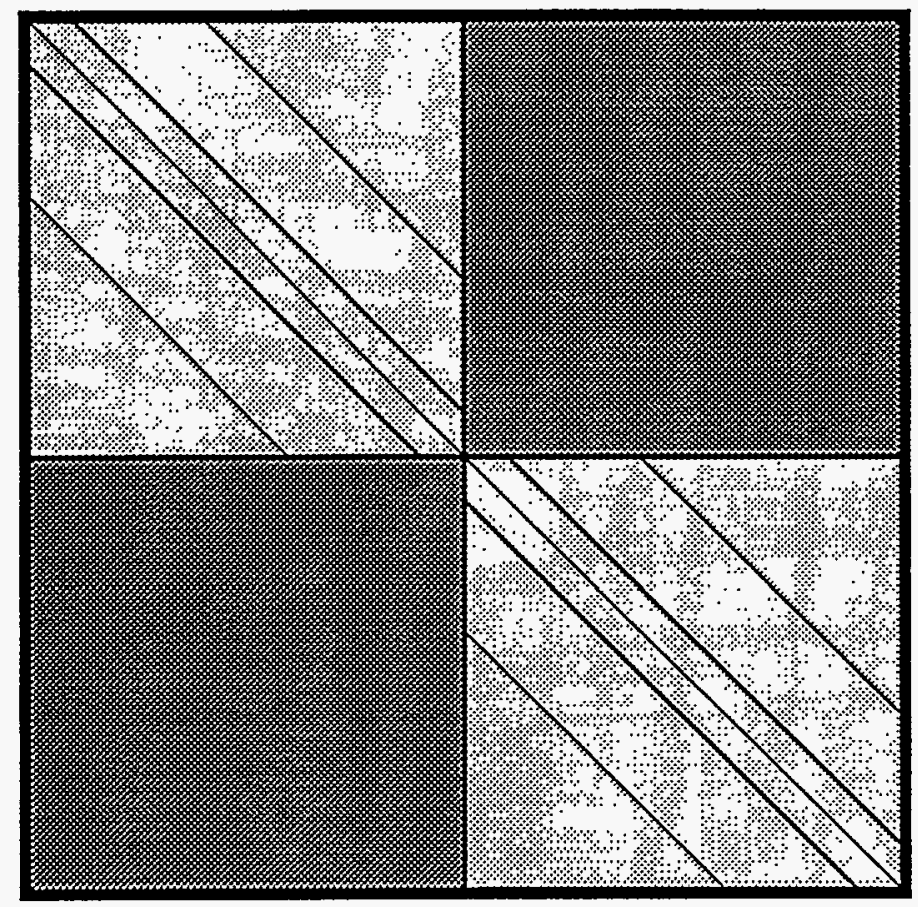

Differential Coupling

Like Coupling

Unlike Coupling

Figure 3.1: Matrix Coupling for Ions and Electrons with Unlike Particle Collisions

Because there are now two equations, the ion VFP equation and the electron VFP equa- 
tion, being solved for at each grid point in $x-v$ space, the new ion-electron matrix has twice as many unknowns as the old ion-only matrix. The new matrix has a block $2 \times 2$ structure where each of the diagonal blocks has the same structure as the ion-only matrix. However, because of the new unlike-particle collision operators, equation. 3.11 and equation. 3.12 , the off diagonal blocks are also filled.

From figure. 3.1 one can see that the coupling between ion and electrons fills in the upper right and lower left corners of the matrix. However, since all of the non-differential coupling is handled by using the matrix-free approximation, this unlike particle coupling does not effect the storage requirements of this solution algorithm. It should be noted that figure. 3.1 is a schematic, and the real matrix structure is not completely full. The real structure is banded with many bands far from the diagonals. The schematic simply represents where the collision operator coupling occurs.

\subsection{Differencing the Fokker-Planck Collision Operator}

Two new methods will be presented for differencing the collision operators, QUICK (Quadratic Upstream Interpolation for Convective Kinematics) [60] and Chang-Cooper [61]. The approach for the two methods is very different. For the QUICK method, the goal is to make all of the error components small so that the total.error will be small. The goal for ChangCooper differencing is to make the errors equal and opposite so the global error is small, due to cancelation. These two methods will now be described in more detail.

\subsubsection{QUICK}

The QUICK (Quadratic Upstream Interpolation for Convective Kinematics) method from 


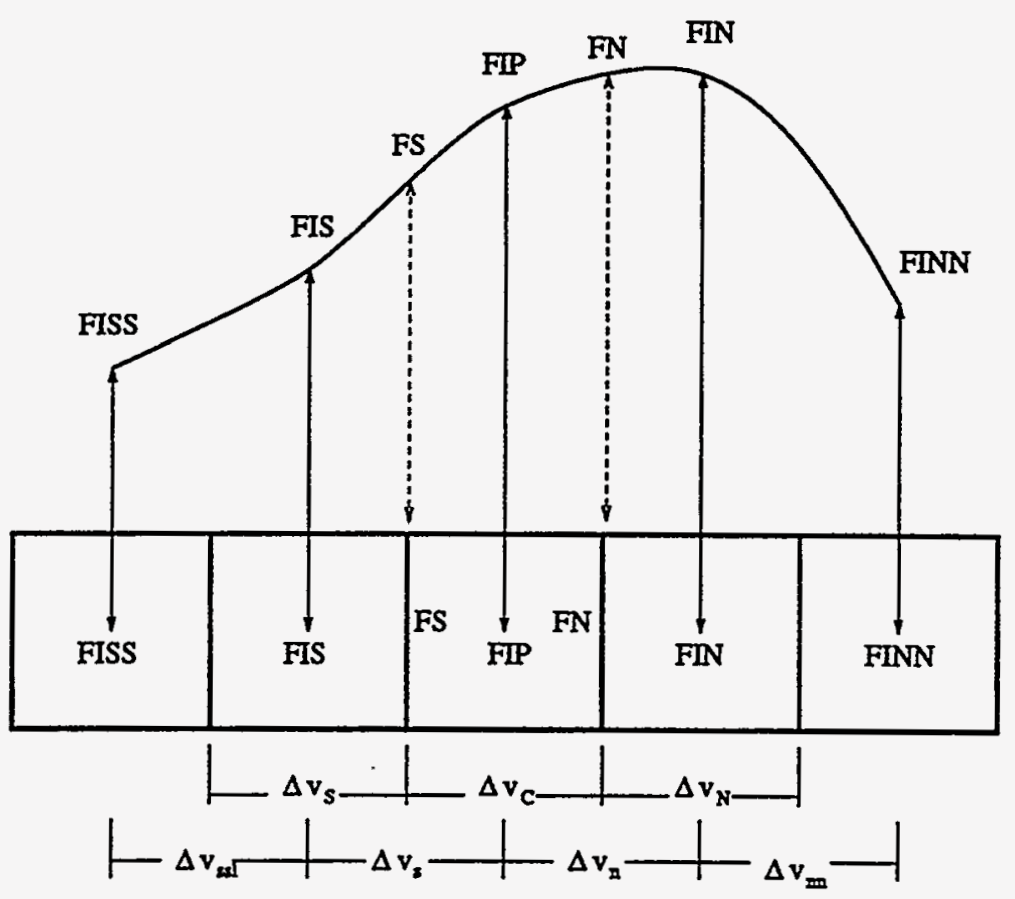

Figure 3.2: QUICK Differencing

Leonard [60] is a third order method for discretizing a convection (first order partial differential $v \frac{\partial F}{\partial v}$ ) diffusion (second order partial differential $D \frac{\partial^{2} F}{\partial v^{2}}$ ) equation. The basic idea is to use four of the five data points (FISS, FIS, FIP, FIN, FINN) shown in figure. 3.2 to calculate the flux values at the two faces, (north-FN and south-FS). The four cell values are chosen by picking the middle three and then the end one in the upwind direction, determined by the fluid velocity.

From these four points, two quadratic equations are computed for the "north" and "south" face. The quadratics are uniquely computed from three cell values. The two cell values that straddle the face are always used (i.e., FIN and FIP for FN) and the upwind cell (i.e., FINN if the velocity at the north face is in the south direction). A quadratic is placed through the three cell values using standard curve fitting logic [62]. This polynomial is then evaluated at the face to give FN. The polynomial is then differentiated with respect to $v$ to evaluate $\frac{\partial F}{\partial v}$ 
at the north face (which is required for the flux terms of the second order operator). This process is then repeated for the south face (FS).

Consider a case where the velocity at the north face, $F N$, is from north to south. Therefore, FIP, FIN, and FINN must be used to construct the curve. Let the grid have its zero value at FIP (i.e., $v_{F I P}=0$ ). Then from Kreyszig [62] an equation for $F(v)$ is,

$$
F(v)=F I P+(v-0) \frac{F I N-F I P}{\Delta v_{n}}+(v-0)\left(v-\Delta v_{n}\right)\left\{\frac{\frac{F I N N-F I N}{\Delta v_{n n}}-\frac{F I N-F I P}{\Delta v_{n}}}{\Delta v_{n n}+\Delta v_{n}}\right\}
$$

Now equation. 3.20 is evaluated at $v=\frac{\Delta v_{n}}{2}$ to get an equation for FN. This yields,

$F\left(\frac{\Delta v_{n}}{2}\right)=F N=F I P+\left(\frac{\frac{\Delta v_{n}}{2}}{\Delta v_{n}}\right)(F I N-F I P)+\left(\frac{\Delta v_{n}}{2}\right)\left(\frac{\Delta v_{n}}{2}-\Delta v_{n}\right)\left\{\frac{\frac{F I N N-F I N}{\Delta v_{n n}}-\frac{F I N-F I P}{\Delta v_{n}}}{\Delta v_{n n}+\Delta v_{n}}\right\}$.

Now a subtle point needs to be discussed before the derivation can continue, the location of the north cell face. There are two obvious choices for this location, $\frac{\Delta v_{c}}{2}$ or $\frac{\Delta v_{n}}{2}$. Clearly if the grid has uniform spacing, then these two choices are equivalent. But if the grid is rapidly stretching or shrinking, these two locations may be very different. To match the derivation of the QUICK algorithm by Leonard [60], the cell faces have to be located half-way between the two cell centers. The geometric interpretation of the other options is that the cell centers are located half-way between the two cell faces. In figure. 3.3 the difference between these two assumptions is clear. Again if the $v=0$ is set at FIP, in the top grid, where the cell center is half-way between the two cell faces, $\mathrm{FN}$ is located at $\frac{\Delta v_{c}}{2}$. In the bottom grid, where the cell face is half-way between the two cell centers, FN is located at $\frac{\Delta v_{n}}{2}$. The bottom grid is the type of grid Leonard used in his derivation, and that will be the type of grid used here to match his derivation. However, in this work the top grid is used, so the implementation 


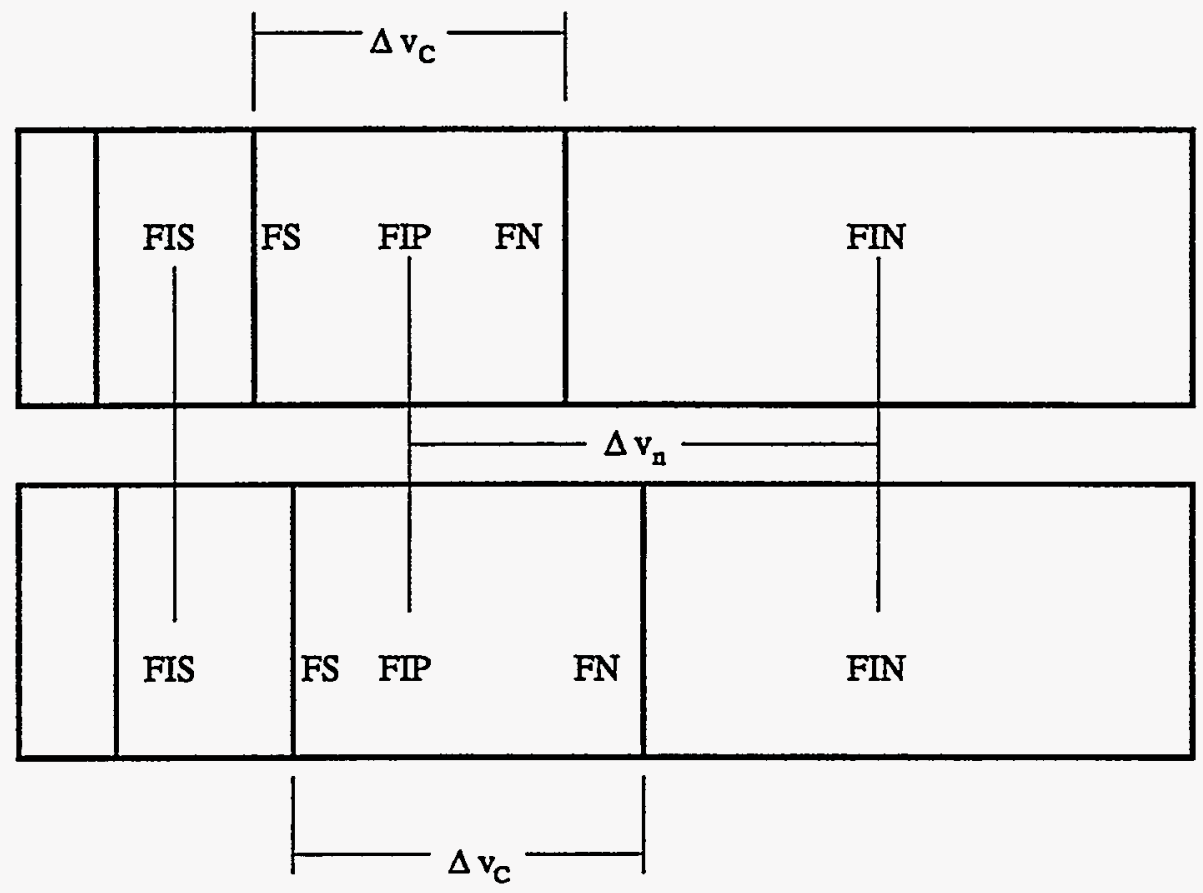

Figure 3.3: Different Non-Uniform Meshes

is slightly different than Leonard's. With a grid of the lower type, the following identity can be used,

$$
\Delta v_{N}=\frac{\Delta v_{n n}+\Delta v_{n}}{2}
$$

From equation. 3.21 , if equation. 3.22 is used and some expressions are simplified the following equation results,

$$
F N=F I P+\frac{(F I N-F I P)}{2}+\frac{1}{2}\left(\frac{\Delta v_{n}}{2}\right)\left(\frac{-\Delta v_{n}}{2}\right)\left\{\frac{\frac{F I N N-F I N}{\Delta v_{n n}}-\frac{F I N-F I P}{\Delta v_{n}}}{\Delta v_{N}}\right\}
$$

One more simplification step results in,

$$
F N=\frac{(F I N+F I P)}{2}-\left(\frac{\Delta v_{n}^{2}}{8}\right)\left\{\frac{\frac{F I N N-F I N}{\Delta v_{n n}}-\frac{F I N-F I P}{\Delta v_{n}}}{\Delta v_{N}}\right\}
$$


This can be seen to match the QUICK algorithm of Leonard [60] equation 9.108. If the mesh is uniform (i.e., all $\Delta v$ 's are equal), one can see that equation. 3.24 reduces to,

$$
F N=\frac{(F I N+F I P)}{2}-\left(\frac{1}{8}\right)\{F I N N-2 F I N+F I P\}
$$

Where it again simplifies to the following:

$$
F N=\frac{1}{8}(3 F I P+6 F I N-F I N N)
$$

This is the equation shown in Johnson and MacKinnon [63] and Leonard [64] for the QUICK method on a uniform mesh.

To complete the differencing discussion, the method for computing $\frac{\partial F}{\partial v}$ needs to be discussed. To set this quantity, one simply has to differentiate equation. 3.20 with respect to $v$ and then evaluate it at $v=\frac{\Delta v_{\mathrm{n}}}{2}$. This differentiation yields:

$$
\frac{\partial F}{\partial v}(v)=\frac{F I N-F I P}{\Delta v_{n}}+\left(2 v-\Delta v_{n}\right)\left\{\frac{\frac{F I N N-F I N}{\Delta v_{n n}}-\frac{F I N-F I P}{\Delta v_{n}}}{\Delta v_{n n}+\Delta v_{n}}\right\} .
$$

When this equation is evaluated at $\frac{\Delta v_{n}}{2}$ the second term is zeroed out and the resulting value is given by:

$$
\frac{\partial F}{\partial v}\left(\frac{\Delta v_{n}}{2}\right)=\frac{F I N-F I P}{\Delta v_{n}}
$$

This matches Leonard's [60] equation (9.109).

Earlier investigations used the Cubic Upwind Interpolation (CUI), given in Johnson and 
MacKinnon [63], for approximating the face values FN, and FS, that is,

$$
F N=\frac{1}{6}(2 F I P+5 F I N-F I N N)
$$

This method was abandoned when the work moved to a non-uniform mesh because the method was no longer conservative (i.e., what flows out of one cell through a face may not equal what flows into the cell on the other side of the face).

\subsubsection{Chang-Cooper}

The Chang-Cooper method, first derived by Chang and Cooper in 1970 [61], is similar to a non-linear version of Interpolated Donor Cell (IDC) differencing (see Subsection 4.3.1 of Chapter 4). The weighting is between forward differencing and central differencing (see Appendix A) instead of between upwind differencing and central differencing. Also, the weighting is now different for each cell and the weighting is determined by local values and an assumed solution shape. This is in contrast to IDC, where the weighting is a globally constant number determined by input.

First consider the collision operator,

$$
\left(\frac{\partial F}{\partial t}\right)_{c}=\nu_{c}\left\{\left[\frac{k T}{m}\right] \frac{\partial^{2} F}{\partial v^{2}}+\frac{\partial[(v-\bar{V}) F]}{\partial v}\right\}=\nu_{c} \frac{\partial}{\partial v}\left\{\left[\frac{k T}{m}\right] \frac{\partial F}{\partial v}+(v-\bar{V}) F\right\}
$$

It will be shown in equation. 3.67 that this continuous collision operator evaluates exactly to zero when $F$ is a continuous Maxwellian distribution,

$$
F_{m}=F_{m 0} \exp \left(\frac{-(v-\bar{V})^{2}}{v_{t h}^{2}}\right)
$$


In the Chang-Cooper method, the discretization is designed to give zero collisions from the discrete collision operator when the discrete distribution function is Maxwellian. Consider the finite volume approximation to the collision operator,

$$
\left.\frac{\partial F}{\partial t}\right|_{j}=\nu_{j} \Delta x\left\{\left[\frac{k T}{m} \frac{\partial F}{\partial v}+(v-\bar{V}) F\right]_{j+1 / 2}-\left[\frac{k T}{m} \frac{\partial F}{\partial v}+(v-\bar{V}) F\right]_{j-1 / 2}\right\}
$$

To evaluate the finite volume discretization of the collision operator, $F_{j+1 / 2}, F_{j-1 / 2}, \frac{\partial F}{\partial v}{ }_{j+1 / 2}$, and $\frac{\partial F}{\partial v}_{j-1 / 2}$ have to be defined. Without loss of generality, one can focus attention on the $j+1 / 2$ terms. To set $\left.\frac{\partial F}{\partial t}\right|_{j}=0$, the discretization scheme will be defined as follows:

$$
\left[\frac{k T}{m} \frac{\partial F}{\partial v}+(v-\bar{V}) F\right]_{j+1 / 2}=\left[\frac{k T}{m} \frac{\partial F}{\partial v}+(v-\bar{V}) F\right]_{j-1 / 2}=0
$$

for a discrete Maxwellian. First, define some terms to make the derivation simpler. Let,

$$
B=(v-\bar{V})
$$

and,

$$
C=\frac{k T}{m}
$$

Now, one can write,

$$
\left[\frac{k T}{m} \frac{\partial F}{\partial v}+(v-\bar{V}) F\right]_{j+1 / 2}=\left[C \frac{\partial F}{\partial v}+B F\right]_{j+1 / 2}
$$


One can also define a discrete Maxwellian distribution function,

$$
F d m_{j}=F d m_{0} \exp \left(\frac{-B(j \Delta v)}{C}\right)
$$

where $F d m_{0}$ has all of the non- $v$-dependent terms and,

$$
v_{j}=j \Delta v
$$

The Chang-Cooper discretization requires that,

$$
\left[C \frac{\partial F d m}{\partial v}+B F d m\right]_{j+1 / 2}=0
$$

First the $\left.\frac{\partial F}{\partial v}\right|_{j+1 / 2}$ is evaluated with a simple central difference,

$$
\left.\frac{\partial F}{\partial v}\right|_{j+1 / 2}=\frac{F_{j+1}-F_{j}}{\Delta v}
$$

The $F_{j+1 / 2}$ is evaluated with a weighted average of two terms,

$$
F_{j+1 / 2}=(1-\delta) F_{j+1}+\delta F_{j}
$$

Now, one can write,

$$
\left[C \frac{\partial F d m}{\partial v}+B F d m\right]_{j+1 / 2}=C\left(\frac{F d m_{j+1}-F d m_{j}}{\Delta v}\right)+B\left((1-\delta) F d m_{j+1}+\delta F d m_{j}\right)=0
$$


This can be rewritten as,

$$
\left[\frac{C}{\Delta v}+B(1-\delta)\right] F d m_{j+1}+\left[B \delta-\frac{C}{\Delta v}\right] F d m_{j}=0
$$

Moving the $F d m$ 's to one side yields,

$$
\frac{F d m_{j+1}}{F d m_{j}}=\frac{-\left(B \delta-\frac{C}{\Delta v}\right)}{\frac{C}{\Delta v}+B(1-\delta)}
$$

From the definition of the discrete Maxwellian, one can write,

$$
\frac{F d m_{j+1}}{F d m_{j}}=\frac{F d m_{0} \exp \left(\frac{-B(j+1 \Delta v)}{C}\right)}{F d m_{0} \exp \left(\frac{-B(j \Delta v)}{C}\right)}=\exp \left(\frac{-B \Delta v}{C}\right)
$$

Therefore, to get the Chang-Cooper method, one simply has to solve the following equation for $\delta$, obtained by equating equation. 3.44 to equation. 3.45 ,

$$
\exp \left(\frac{-B \Delta v}{C}\right)=\frac{-\left(B \delta-\frac{C}{\Delta v}\right)}{\frac{C}{\Delta v}+B(1-\delta)}
$$

To solve this equation, first let $w=\frac{B \Delta v}{C}$, multiply through by the Right Hand Side (RHS) denominator, and multiply the numerator through by -1 .

$$
\exp (-w)\left(\frac{C}{\Delta v}+B(1-\delta)\right)=\frac{C}{\Delta v}-B \delta
$$

Multiplying out yields,

$$
\exp (-w) \frac{C}{\Delta v}+\exp (-w) B-\exp (-w) B \delta=\frac{C}{\Delta v}-B \delta
$$


Gathering up the $\delta$ terms gives,

$$
B \delta(1-\exp (-w))=\frac{C}{\Delta v}(1-\exp (-w))-\exp (-w) B
$$

Solving for $\delta$ results in,

$$
\delta=\frac{C}{\Delta v B}-\frac{\exp (-w)}{(1-\exp (-w))}
$$

Recalling the definition of $w$ and multiplying the second term on the RHS by $\frac{\exp (w)}{\exp (w)}$, one gets,

$$
\delta=\frac{1}{w}-\frac{1}{\exp (w)-1}
$$

which is equation 18 of Chang and Cooper's paper [61].

\subsection{Model Problem and Results}

In this section results will be presented for three model problems, a calculation of resistivity, a null problem, and a thermal equilibrium problem. For the thermal equilibrium problem, where the ions and electrons are initialized to two distinct temperatures, one can define,

$$
\Delta T=T_{e}-T_{i}
$$

It should be noted that Catto [8] restricts the simplified collision operator to the regime where,

$$
\begin{aligned}
|\varepsilon| & \ll \frac{T_{e}}{m_{e}} \\
\left|\bar{V}_{i}-\bar{V}_{e}\right| & \ll\left|\bar{V}_{e}\right|
\end{aligned}
$$


where $\varepsilon$ is defined in equation. 3.13. Note that equation. 3.54 is the last assumption made by Braginskii in Section 1.2 when he derived his Brownian collision operator. These restrictions limit the choices for temperature and velocity differences.

\subsubsection{Exact Solution for Resistivity Calculation}

The following two equations for resistivity of a plasma come from Chen [12],

$$
\nu_{e i}=\frac{n_{e} e^{2}}{m_{e}} \eta
$$

where $\eta$ is the specific resistivity, and,

$$
E=\eta j
$$

which is a simplified statement of Ohm's law. Here $j$ is the current:

$$
j=e n_{e}\left[\bar{V}_{i}-\bar{V}_{e}\right]
$$

Solving equation. 3.55 and equation. 3.56 for $\eta$, and setting the two equations equal yields,

$$
\frac{E}{j}=\frac{\nu_{e i} m_{e}}{n_{e} e^{2}}
$$

Substituting in equation. 3.57 for $j$ and solving for $\left[\bar{V}_{i}-\bar{V}_{e}\right]$ gives,

$$
\Delta V=\left[\bar{V}_{i}-\bar{V}_{e}\right]=\frac{e E}{m_{e} \nu_{e i}}
$$




\subsubsection{Results for Resistivity Calculation}

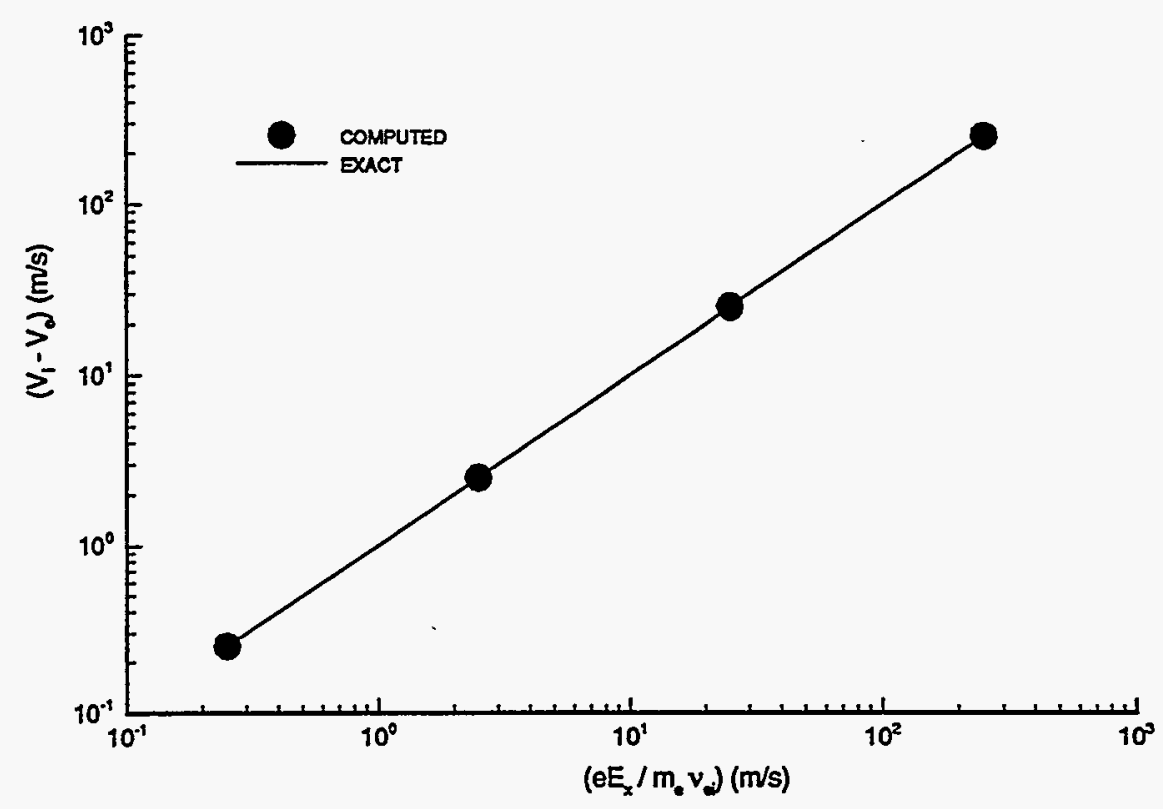

Figure 3.4: Check of Exact Solution of Resistivity

Figure. 3.4 shows the results from the numerical simulation versus the exact solution from equation. 3.59. This plot was made by running four transient runs with different electric field strengths, $E$, and then plotting out the the $\Delta V$ values that resulted. From this figure one can see that the code values for $\Delta V$ are in very good agreement with the exact solution. Figure. 3.5 shows the velocity transient for $\Delta V_{\text {exact }}=250$. Here, one can see the electric field accelerating the ions up to $2.48 \mathrm{~m} / \mathrm{s}$, and the electrons to $-247.52 \mathrm{~m} / \mathrm{s}$. From the plot, it can be seen that the transient is almost completed in the first $2.0 \times 10^{-6}$ seconds.

\subsubsection{Exact Solution Null Problem (Maxwellian)}

For the null problem, one simply initializes velocity space to a Maxwellian distribution at a given temperature, number density, and velocity. This problem tests the codes ability to hold 


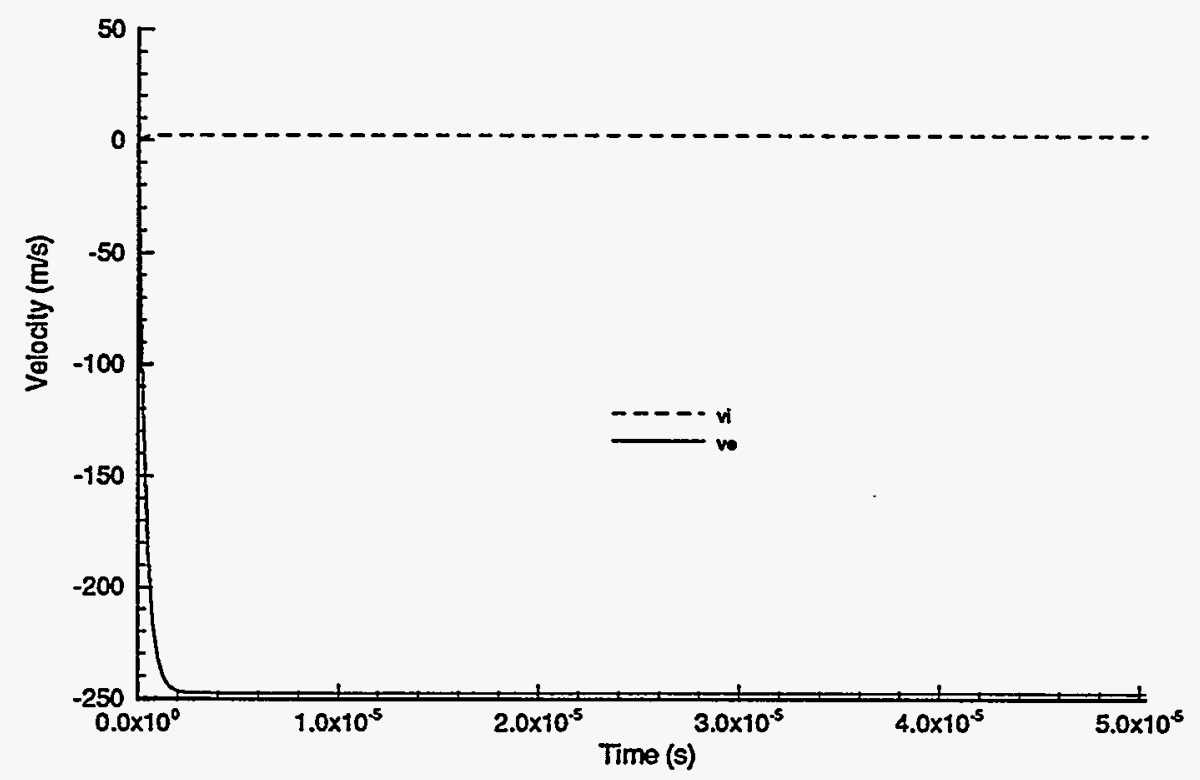

Figure 3.5: Velocity Transients for Resistivity Calculations

the correct solution without drifting away. The input values used are shown in Table 3.1. Although this problem seems trivial, it can be a difficult one to solve, as is shown in Chapter 5

\begin{tabular}{|c|c|c|c|c|c|}
\hline \hline$n x$ & 1 & $n y$ & 200 & $T_{0}$ & $30.0 \mathrm{eV}$ \\
\hline$V_{0}$ & $0.0 \mathrm{~m} / \mathrm{s}$ & $v_{\max }$ & $2.0 \times 10^{5} \mathrm{~m} / \mathrm{s}$ & $n_{0}$ & $1.0 \times 10^{20} \mathrm{~m}^{-3}$ \\
\hline$\nu_{c}$ & $2.5 \times 10^{4} \mathrm{~s}^{-1}$ & $t_{\text {final }}$ & $50 \times \nu_{c}^{-1}$ & $d t$ & $4.0 \times 10^{-6} s$ \\
\hline \hline
\end{tabular}

Table 3.1: Null Problem Input Values

of Coster's thesis [38]. This problem displays the numerical discretization's ability to exactly represent the collisional operator on a discrete mesh.

To appreciate the difficulty, one first needs to understand how the collision operator works 
in continuous form. Consider first a Maxwellian distribution function,

$$
F_{m}=n\left(\frac{m}{2 k T \pi}\right)^{1 / 2} \exp \left(\frac{-m(v-\bar{V})^{2}}{2 k T}\right)
$$

Recall that $v_{t h}^{2}=\frac{2 k T}{m}$ and let $F_{m 0}=n\left(\frac{1}{v_{t h}^{2} \pi}\right)^{1 / 2}$ then,

$$
F_{m}=F_{m 0} \exp \left(\frac{-(v-\bar{V})^{2}}{v_{t h}^{2}}\right)
$$

Recall equation. 2.8 from Chapter 2,

$$
\left(\frac{\partial F}{\partial t}\right)_{c}=\nu_{c}\left\{\left[\frac{k T}{m}\right] \frac{\partial^{2} F}{\partial v^{2}}+\frac{\partial[(v-\bar{V}) F]}{\partial v}\right\}
$$

To evaluate this function exactly, some preliminary steps must taken. First consider,

$$
\frac{\partial F_{m}}{\partial v}=\frac{-2 F_{m 0}}{v_{t h}^{2}}(v-\bar{V}) \exp \left(\frac{-(v-\bar{V})^{2}}{v_{t h}^{2}}\right)=\frac{-2}{v_{t h}^{2}}(v-\bar{V}) F_{m}
$$

and,

$$
\frac{\partial^{2} F_{m}}{\partial v^{2}}=\frac{\partial}{\partial v}\left(\frac{-2}{v_{t h}^{2}}(v-\bar{V}) F_{m}\right)=\frac{-2}{v_{t \hbar}^{2}}\left(F_{m}+(v-\bar{V}) \frac{\partial F_{m}}{\partial v}\right)
$$

Substituting for $\frac{\partial F_{m}}{\partial v}$, one gets:

$$
\frac{\partial^{2} F_{m}}{\partial v^{2}}=\frac{-2}{v_{t h}^{2}}\left(1+\frac{-2}{v_{t h}^{2}}(v-\bar{V})^{2}\right) F_{m}
$$

From the chain rule,

$$
\frac{\partial\left[(v-\bar{V}) F_{m}\right]}{\partial v}=F_{m}+(v-\bar{V}) \frac{\partial F_{m}}{\partial v}=\left(1+\frac{-2}{v_{t h}^{2}}(v-\bar{V})^{2}\right) F_{m}
$$


Recalling that $k T / m=v_{t h}^{2} / 2$, the Fokker-Planck operator evaluated for a Maxwellian distribution now becomes,

$$
\left(\frac{\partial F_{m}}{\partial t}\right)_{c}=\nu_{c}\left\{\left[\frac{v_{t h}^{2}}{2}\right] \frac{-2}{v_{t h}^{2}}\left(1+\frac{-2}{v_{t h}^{2}}(v-\bar{V})^{2}\right) F_{m}+\left(1+\frac{-2}{v_{t h}^{2}}(v-\dot{V})^{2}\right) F_{m}\right\}=0
$$

To see why equation. 3.67 is difficult to handle numerically, consider some typical numerical values in Table 3.2 .

\begin{tabular}{|c|c|c|c|}
\hline \hline$n$ & $1.0 \times 10^{20} \mathrm{~m}^{-3}$ & $m$ & $3.32 \times 10^{-27} \mathrm{~kg}$ \\
\hline$k$ & $1.60 \times 10^{-19} \mathrm{~J} / \mathrm{eV}$ & $T$ & $30 \mathrm{eV}$ \\
\hline$v_{t h}^{2}$ & $2.89 \times 10^{9} \mathrm{~m}^{2} / \mathrm{s}^{2}$ & $F_{m 0}$ & $2.43 \times 10^{17} \mathrm{~s} / \mathrm{m}^{4}$ \\
\hline$v$ & $5.376 \times 10^{4} \mathrm{~m} / \mathrm{s}$ & $v_{t h}$ & $5.376 \times 10^{4} \mathrm{~m} / \mathrm{s}$ \\
\hline$F_{m}$ & $8.94 \times 10^{16} \mathrm{~s} / \mathrm{m}^{4}$ & $\bar{V}$ & 0 \\
\hline$k T / m$ & $1.445 \times 10^{9} \mathrm{~m}^{2} / \mathrm{s}^{2}$ & $\nu_{c}$ & $2.5 \times 10^{4} \mathrm{~s}^{-1}$ \\
\hline$\frac{\partial^{2} F_{m}}{\partial v^{2}}$ & $6.19 \times 10^{7} \mathrm{~s}^{3} / \mathrm{m}^{6}$ & $\frac{\partial v \mathcal{F}_{m}}{\partial v}$ & $-8.94 \times 10^{16} \mathrm{~s} / \mathrm{m}^{4}$ \\
\hline \hline
\end{tabular}

Table 3.2: Typical Numerical Maxwellian Input Values

Numerically equation. 3.62 would be,

$$
\left(\frac{\partial F_{m}}{\partial t}\right)_{c}=\left(2.5 \times 10^{4}\right)\left\{\left[1.445 \times 10^{9}\right]\left(6.19 \times 10^{7}\right)+\left(-8.94 \times 10^{16}\right)\right\}=1.1375 \times 10^{18} \neq 0 .
$$

Equation. 3.68 demonstrates the numerical sensitivity of the Fokker-Planck collision operator. In trying to evaluate the closed form solution, differences of a fraction of a percent, in terms which should cancel, lead to large errors.

Now consider a numerical implementation. Whenever a differential operator is approximated numerically, an error is introduced. Let $D 2 F D V 2$ be the numerical approximation to 
$\frac{\partial^{2} F}{\partial v^{2}}$ and $D F D V$ be the numerical approximation to $\frac{\partial F}{\partial v}$. One can write,

$$
\begin{aligned}
D 2 F D V 2 & =\frac{\partial^{2} F}{\partial v^{2}}+\left.\frac{\partial^{2} F}{\partial v^{2}}\right|_{e r r} \\
D F D V & =\frac{\partial F}{\partial v}+\left.\frac{\partial F}{\partial v}\right|_{e r r} .
\end{aligned}
$$

Substituting these expressions into the Fokker Planck collision operator with values from Table 3.2 and cancelling out the exact terms, one gets the following:

$$
\left.\left(\frac{\partial F_{m}}{\partial t}\right)_{c}\right|_{e r r}=\left(2.5 \times 10^{4}\right)\left\{\left.\left[1.445 \times 10^{9}\right] \frac{\partial^{2} F}{\partial v^{2}}\right|_{e r r}+\left.\frac{\partial F}{\partial v}\right|_{e r r}\right\} .
$$

This again demonstrates the problems with the numerical solution of the Fokker Planck equation, since one can see that the discretization error of the second order term, $\left.\frac{\partial^{2} F}{\partial v^{2}}\right|_{\text {err }}$, is multiplied by a large number, $3.6125 \times 10^{13}$.

\subsubsection{Results for the Null Problem (Maxwellian)}

Numerical demonstrations of these sensitivity phenomenon follow. Using a finite volume discretization, one approximates the differentials as follows,

$$
\frac{k T}{m} \frac{\partial^{2} F}{\partial v^{2}}+\frac{\partial[(v-\bar{V}) F]}{\partial v} \approx \Delta x\left(\frac{k T}{m}\left[\left.\frac{\partial F}{\partial v}\right|_{N}-\left.\frac{\partial F}{\partial v}\right|_{S}\right]+\left[\left.\{(v-\bar{V}) F\}\right|_{N}-\left.\{(v-\bar{V}) F\}\right|_{S}\right]\right)
$$

In the following plots various terms from equation. 3.70 will be plotted. Figure. 3.6 shows the value of equation. 3.70 as a function of particle velocity. For this plot, upwind differencing (see Section 4.2) was used to approximate $\frac{\partial F}{\partial v}$ and central differencing (see Appendix A) was used to approximate $\frac{\partial^{2} F}{\partial v^{2}}$. The finite volume operator was evaluated with a discrete Maxwellian 


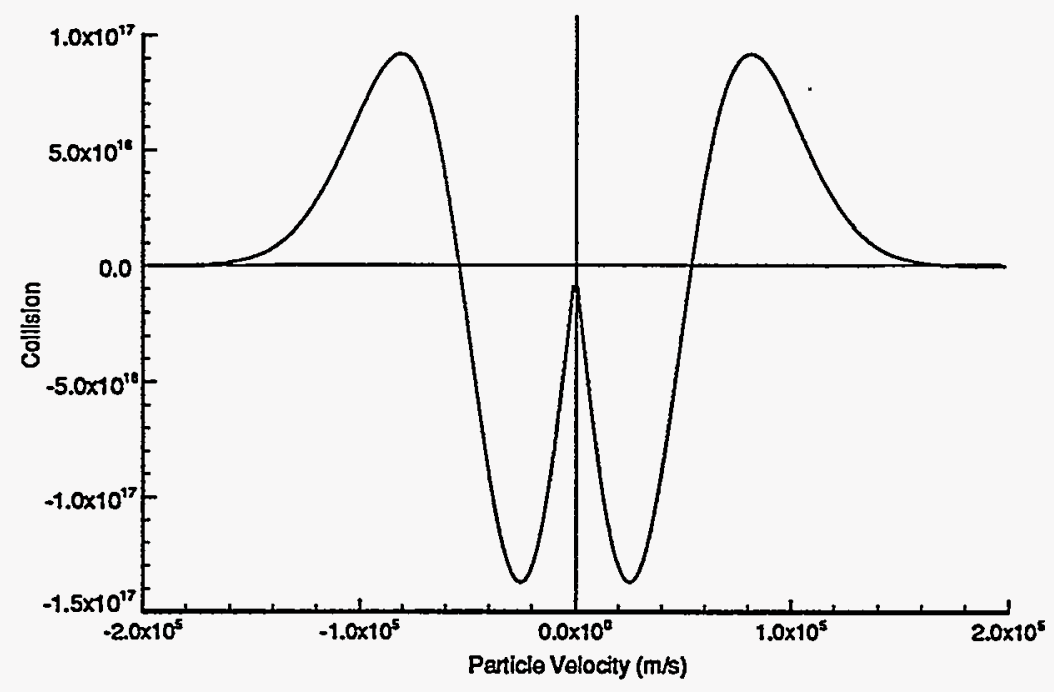

Figure 3.6: Upwind Differencing 200 Cells Initial Collision Operator Values

distribution function (see Table 3.1 for conditions). The exact solution is zero for all particle velocities. Here, one can see that the maximum error is about $1.0 \times 10^{17}$. Also note that the plot is very structured. This structure is due to the following three conservation laws.

- Conservation of mass dictates that the area above the Collision $=0$ line and the area below must be equal. For every place that mass is being added, mass is being removed somewhere else.

- Conservation of momentum requires that the plot be symmetric about the zero particle velocity line. This guarantees that the integral of the collision operator is zero and no momentum is added or subtracted by like particle collisions.

- Conservation of energy would be maintained by the plot being zero everywhere.

It appears that this discretization technique did well on conservation of mass and momentum but did not conserve energy. The units of Collision are $s / m^{4}$ so it is not obvious how much 
energy will be added or lost. This energy conservation question will be presented later, but first the source of the error will be investigated.

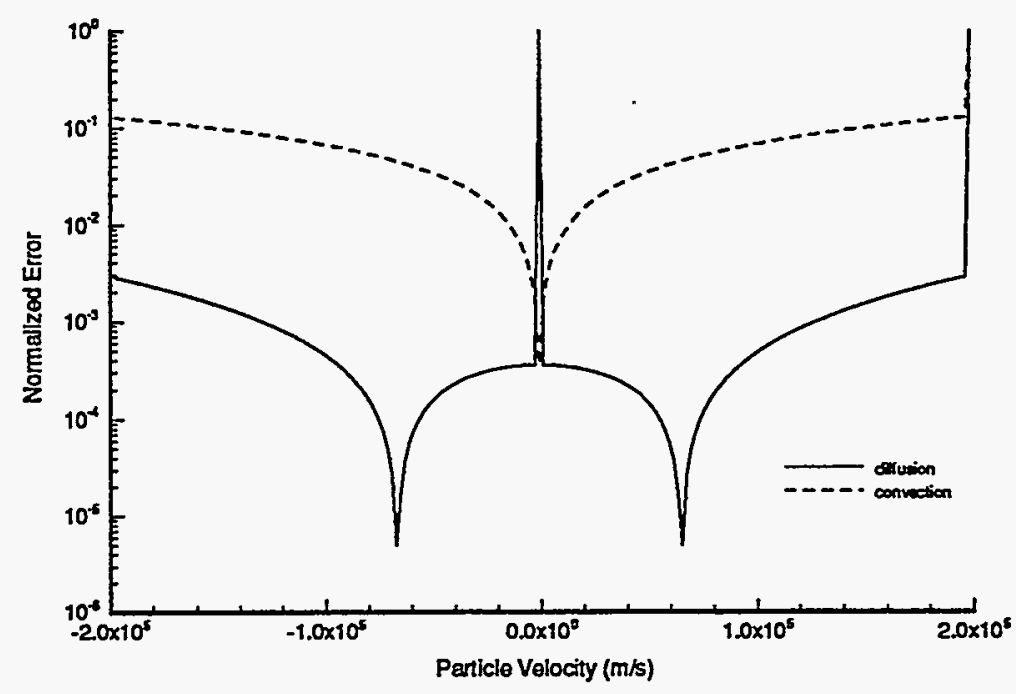

Figure 3.7: Upwind Differencing 200 Cells Initial Normalized Error

Figure. 3.7 shows the normalized error as a function of particle velocity for upwind differencing of the convective, or friction, part of the collision operator. The two lines on the plot represent the normalized error of the convection $\left(\left.\{(v-\bar{V}) F\}\right|_{N}\right)$ and the diffusion $\left(\left.\frac{k T}{m} \frac{\partial F}{\partial v}\right|_{N}\right)$ terms in equation. 3.70. The normalization is defined by $\left|\frac{\text { exact-calculated }}{\text { exact }}\right|$, where the exact values are provided by equation. 3.61 and equation. 3.63 .

There are two spikes in this plot, one near particle velocity of zero and one near vmax $=$ $2.0 \times 10^{5}$. The normalized error at both of these points is one. This is caused by the computed solution being zero and the exact solution not being zero. Near $v=v m a x$, the spike is caused by the computed term being set equal to zero because of boundary conditions. Near $v=0$ the correct value is zero but due to round-off errors the exact value does not equal zero. If it did, the value of the normalized error would be undefined. 
If the two spikes described above are ignored, one can see that figure. 3.6 is the result of the difference between the error in the convection and diffusion terms of figure. 3.7, multiplied by a normalization constant. Because of the normalization constant and the absolute value in the definition of the normalized error, one cannot directly reconstruct figure. 3.6 from figure. 3.7. To help prove this point, the same two plots will be shown after 50 collision times.

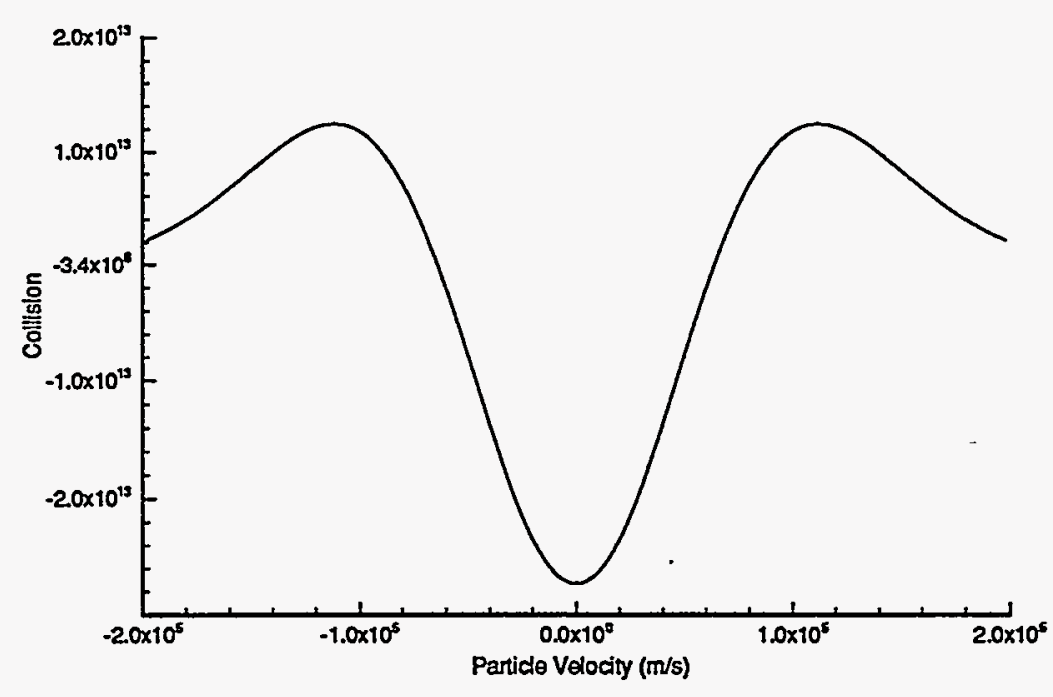

Figure 3.8: Upwind Differencing 200 Cells Final Collision Operator Values

Figure. 3.8 shows the value of equation. 3.70 after the transient has evolved to a time of $\frac{50}{\nu_{c}}$ seconds. Here one quickly notes that the maximum error has been reduced from approximately $1.0 \times 10^{17}$ to $2.0 \times 10^{13}$ or about four orders of magnitude. When one examines figure. 3.9, it is clear that the normalized errors in the convective term and the diffusive term are now equal, since the two curves lay together. These equal values in. error were accomplished by decreasing the error in the convective term and by increasing the error in the diffusive term. It appears that the transient has evolved by driving equation. 3.69 to 


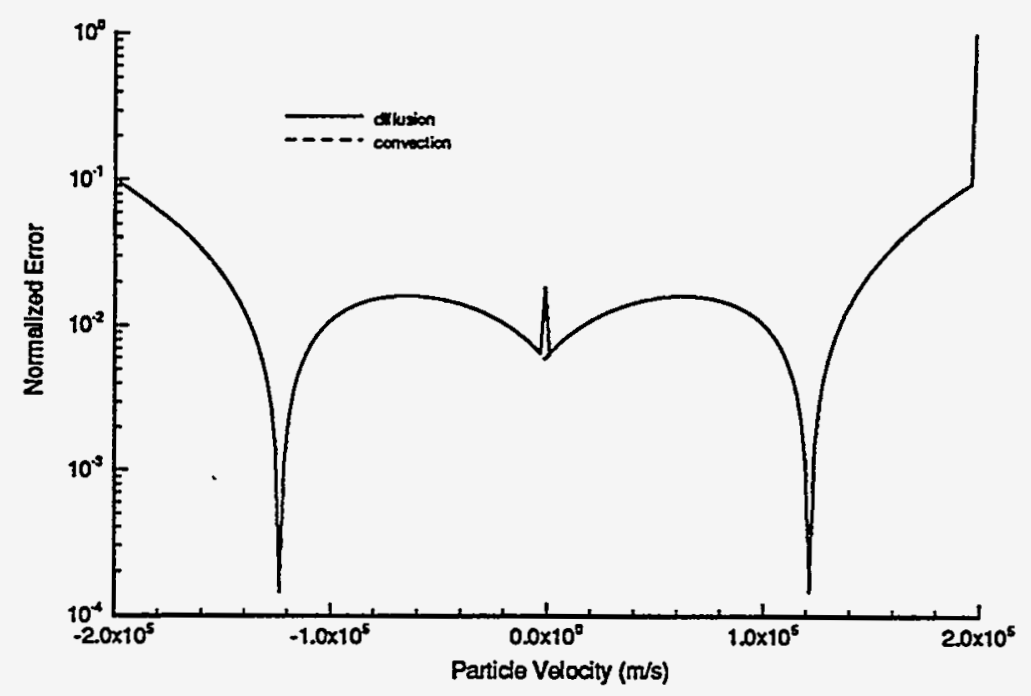

Figure 3.9: Upwind Differencing 200. Cells Final Normalized Error

zero. Figure. 3.10 shows the transient temperature for 50 collision times. Because of the large values initially in equation. 3.70 , the simulation followed a transient that heated the plasma until the convective and diffusive discretization errors cancelled. The correct solution is for the temperature to stay at $30 \mathrm{eV}$, but one can see that the temperature has warmed to about $85 \mathrm{eV}$ in about 20 collision times. The upwind scheme can be made better by using a larger number of cells, approximately 3200 instead of 200 , but the amount of work to carry this many velocity cells for a two dimensional simulation is prohibitive.

To eliminate the need for such a large number of cells, higher order differencing schemes were investigated. In particular the QUICK scheme of Leonard [60] was used. The idea here is to make the error in the convective term and the error in the diffusion term both so small that the contribution from their difference is negligible. In figure. 3.11, one can see the QUICK plot that corresponds to figure. 3.6 for the upwind scheme, that is the initial values of equation. 3.70 when it is given a Maxwellian initial guess. Comparing these two 


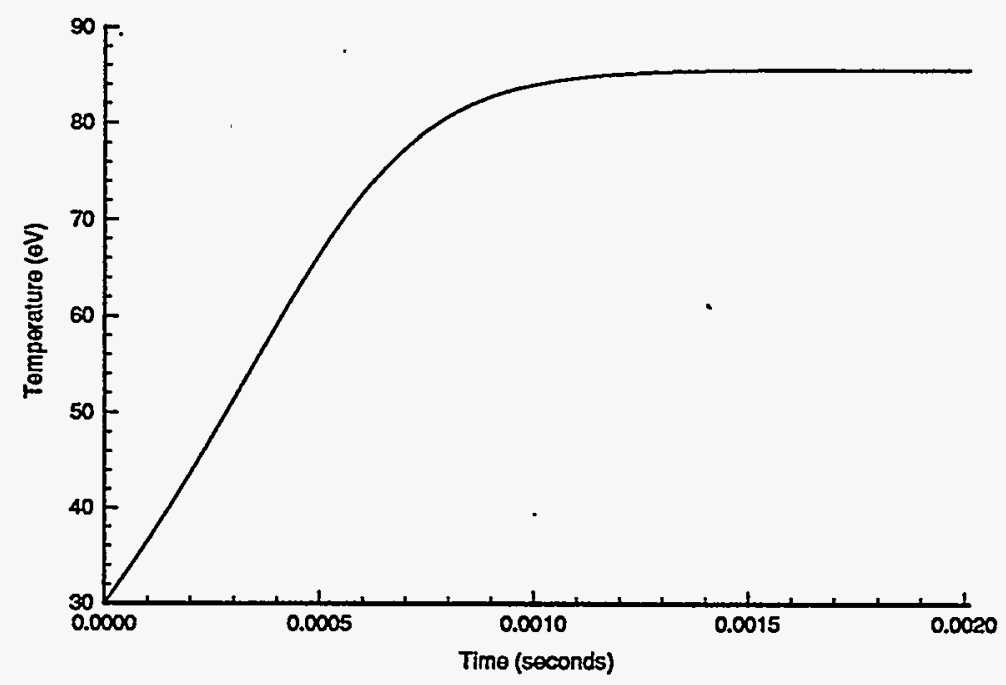

Figure 3.10: Upwind Differencing 200 Cells Temperature Transient

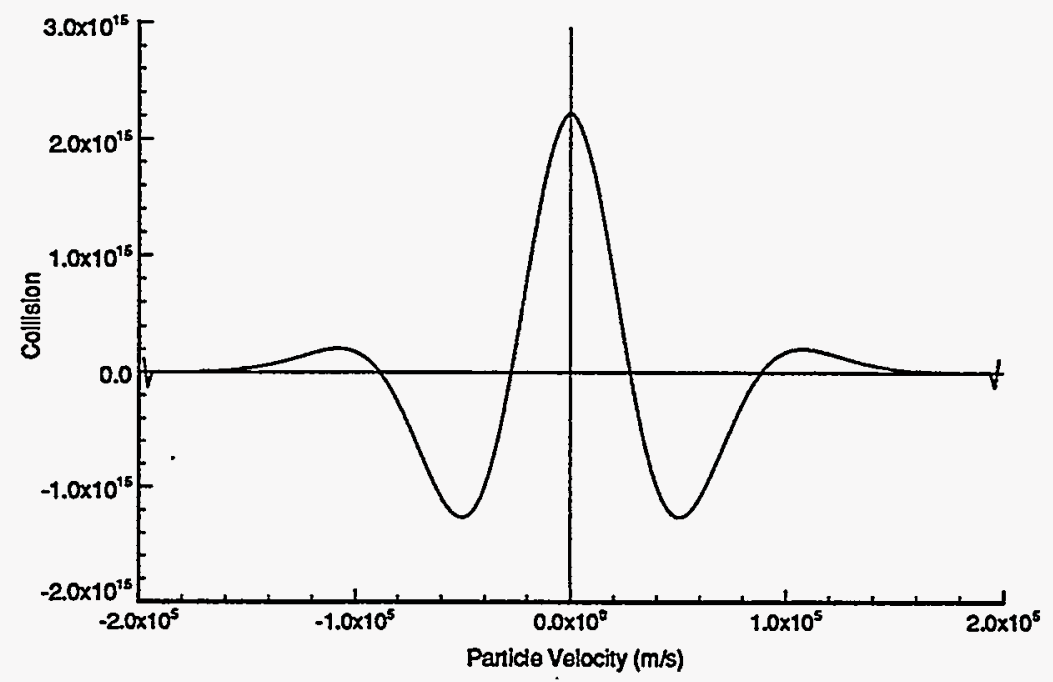

Figure 3.11: Quick Differencing 200 Cells Initial Collision Operator Values 
figures, the error in the QUICK scheme is about two orders of magnitude smaller for the same number of cells. Figure. 3.12 shows why this error is smaller. Comparing figure. 3.12

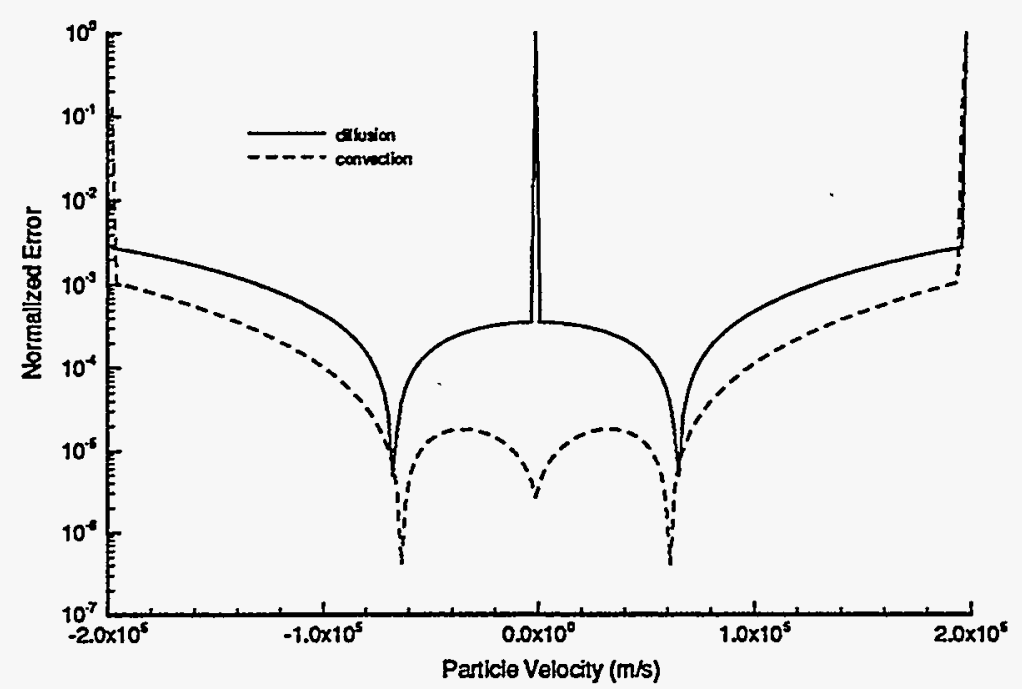

Figure 3.12: Quick Differencing 200 Cells Initial Normalized Error

to figure. 3.7 for the upwind scheme, one notes that the initial error in the convective terms are much smaller for the QUICK method than for upwind differencing. In particular, in the QUICK scheme the.convective error is smaller than the diffusion error while for upwind differencing the convective error is much larger than the diffusion error. When one looks at the temperature transient displayed in figure. 3.13 , it is clear that in 50 collision times the temperature has only dropped $0.06 \mathrm{eV}$. Therefore, it appears for short time scale problems that the QUICK method is acceptable. What the plot does not show is that as the simulation time increases the temperature continues to fall until it becomes a problem. For the single species problem with short time scales, the QUICK method may be sufficient, but for the ion-electron problems where there are four collision operators, two like-particle collisions and two unlike-particle collisions, the QUICK method may not be accurate enough. The 


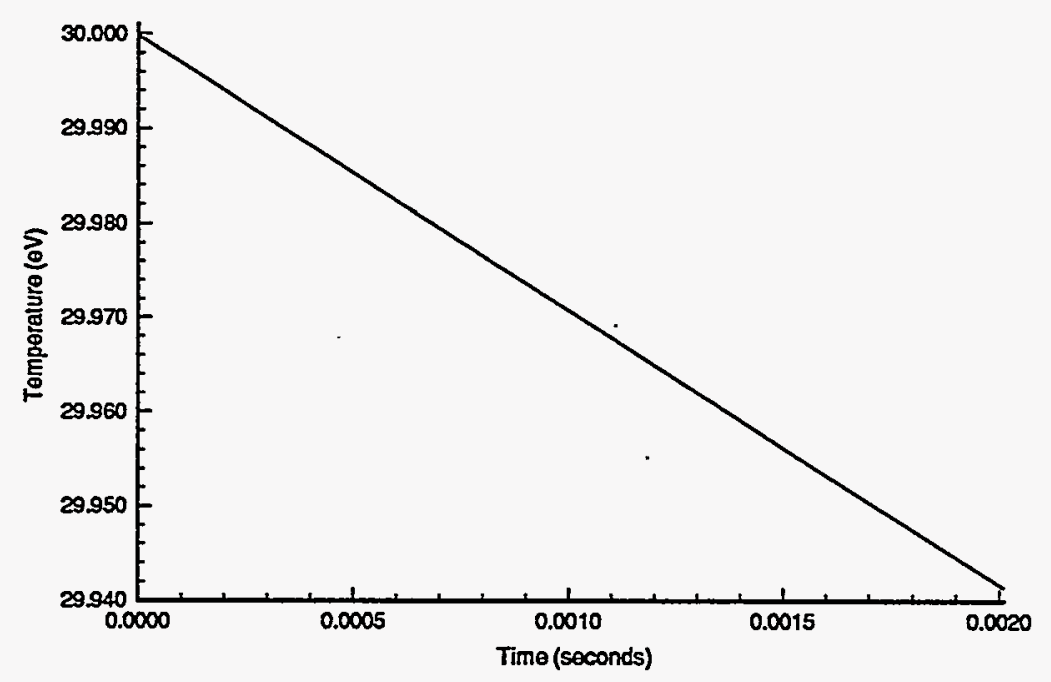

Figure 3.13: Quick Differencing 200 Cells Temperature Transient

collision frequencies of the different collision operators vary with the mass ratio of the ions and electrons as can be seen in equation. 3.14 through equation. 3.17. If one uses a simulation ion-electron mass ratio of 100 (recall that the correct mass ratio is about 1800) and plans to simulate 20 collision times of the slow collision rate (ion-electron, $\nu_{i e}$ ), then the simulation will run for 2000 collision times for the fast rate (electron-electron, $\nu_{e e}$ ). In this large number of collision times, even a small error growth rate will become a problem.

For this problem, the ideal method would set the convection and diffusion errors equal and opposite to each other, which would result in no artificial transient at all. This is the basic approach of the Chang-Cooper method [61]. In 1985, Larsen [65] pointed out some of the shortcomings of this method and also presented a more general equation. Even after this work, the Chang-Cooper method has had continual use for many years [35,66-68]. In figure. 3.14 the values of equation. 3.70 are shown for the Chang-Cooper method of discretization. The maximum error for the Chang Cooper method is five orders of magnitude smaller than the 


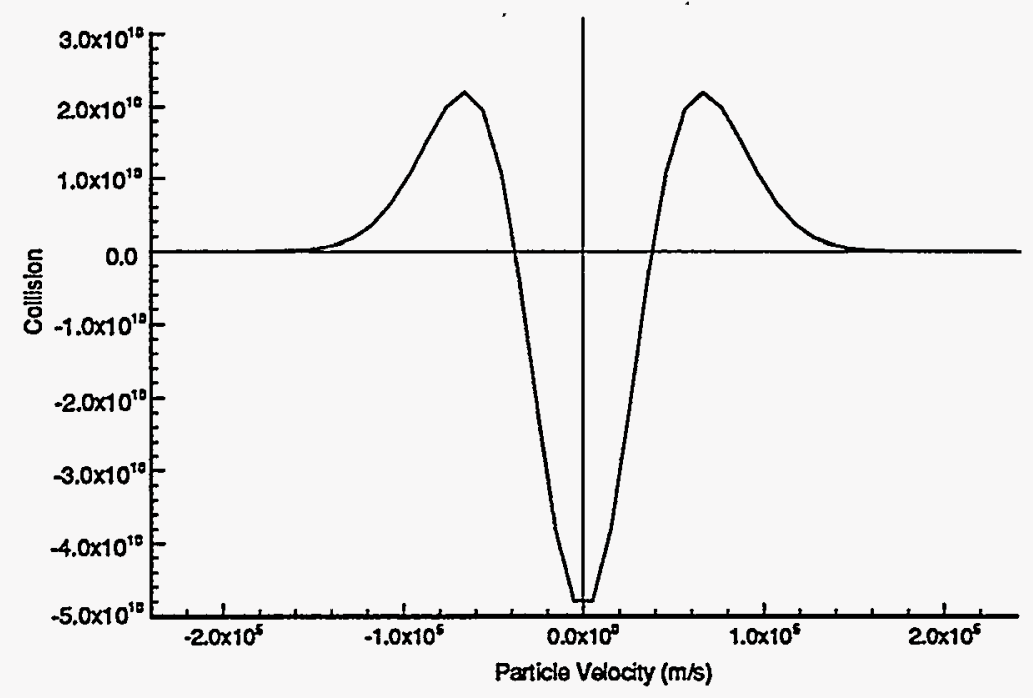

Figure 3.14: Chang-Cooper IDC Differencing 50 Cells Initial Collision Operator Values maximum error for the QUICK method with four times as many cells, 50 versus 200 . It should also be noted that vmax has been raised from $4 v_{t h}$ to $5 v_{t h}$, which was done to increase the accuracy of the Chang-Cooper method. The same increase in vmax decreased the accuracy in the QUICK method. Figure. 3.15 displays the source of this accuracy. It can easily be seen from comparing figure. 3.12 and figure. 3.15 that the error in the convection term and the diffusion term are both much larger than when using the QUICK scheme, but they are exactly equal except for the two points where the terms are zero. Figure. 3.16 demonstrates that energy is well conserved if there is no initial source of perturbation to cause it to change.

\subsubsection{Exact Solution for Thermal Equilibrium}

The last test problem shown in this chapter is a thermal equilibrium problem. The ions and the electrons are initialized to two different temperatures and are allowed to relax. To find the correct relation rate (i.e., the rate that $\Delta T \rightarrow 0$ ) the third moment of the VFP 


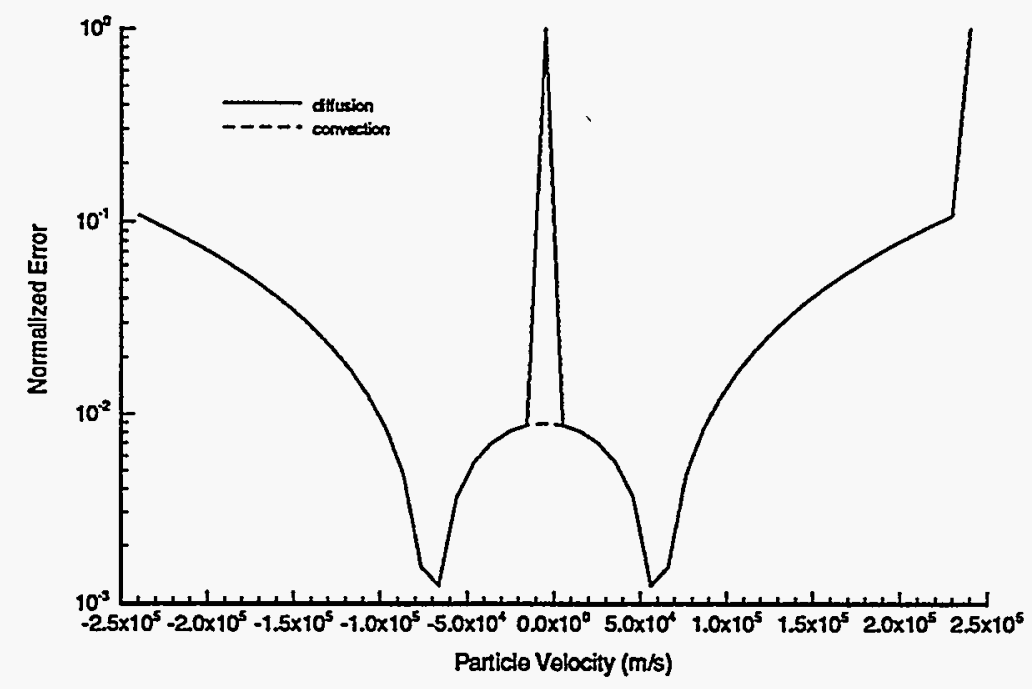

Figure 3.15: Chang-Cooper IDC Differencing 50 Cells Initial Normalized Error

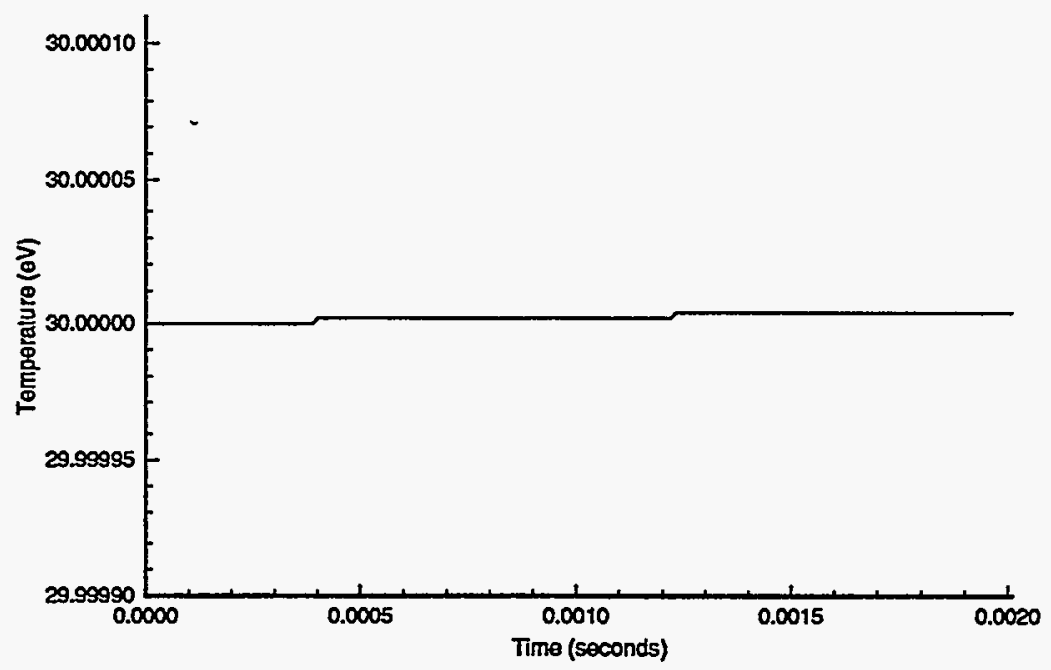

Figure 3.16: Chang-Cooper IDC Differencing 50 Cells Temperature Transient 
equation (i.e., the conservation of energy equation) must be computed. To accomplish this the third moment of the ion-electron collision operator, equation. 3.11 , has to be computed. This becomes,

$$
E Q I B=\int_{-\infty}^{\infty} \frac{1}{2} m_{i} v_{i}^{2}\left[\left(\frac{\partial F_{i}}{\partial t}\right)_{i e}\right] d v_{i}
$$

Substituting in equation. 3.11 yields,

$$
E Q I B=\int_{-\infty}^{\infty} \frac{1}{2} m_{i} v_{i}^{2}\left[\nu_{i e}\left\{\left[\frac{k T_{e}}{m_{i}}\right] \frac{\partial^{2} F_{i}}{\partial v_{i}^{2}}+\frac{\partial\left[\left(v_{i}-\bar{V}_{e}\right) F_{i}\right]}{\partial v_{i}}\right\}\right] d v_{i}
$$

Substituting in equation. 3.16 one gets,

$$
E Q I B=\int_{-\infty}^{\infty} \frac{1}{2} m_{i} v_{i}^{2}\left[\left(\frac{m_{e} n_{e}}{m_{i} n_{i}} \nu_{e i}\right)\left\{\left[\frac{k T_{e}}{m_{i}}\right] \frac{\partial^{2} F_{i}}{\partial v_{i}^{2}}+\frac{\partial\left[\left(v_{i}-\bar{V}_{e}\right) F_{i}\right]}{\partial v_{i}}\right\}\right] d v_{i}
$$

Combining terms results in the following:

$$
E Q I B=\left(\frac{m_{e} n_{e} \nu_{e i}}{2 n_{i}}\right) \int_{-\infty}^{\infty} v_{i}^{2}\left[\left\{\left[\frac{k T_{e}}{m_{i}}\right] \frac{\partial^{2} F_{i}}{\partial v_{i}^{2}}+\frac{\partial\left[\left(v_{i}-\bar{V}_{e}\right) F_{i}\right]}{\partial v_{i}}\right\}\right] d v_{i}
$$

Rewriting the partial derivatives gives one the following:

$$
E Q I B=\left(\frac{m_{e} n_{e} \nu_{e i}}{2 n_{i}}\right) \int_{-\infty}^{\infty} v_{i}^{2} \frac{\partial}{\partial v_{i}}\left[\left\{\left[\frac{k T_{e}}{m_{i}}\right] \frac{\partial F_{i}}{\partial v_{i}}+\left[\left(v_{i}-\bar{V}_{e}\right) F_{i}\right]\right\}\right] d v_{i} .
$$

Integrating by parts,

$$
\begin{aligned}
E Q I B & =\left(\frac{m_{e} n_{e} \nu_{e i}}{2 n_{i}}\right)\left\{\left.v_{i}^{2}\left[\left\{\left[\frac{k T_{e}}{m_{i}}\right] \frac{\partial F_{i}}{\partial v_{i}}+\left[\left(v_{i}-\bar{V}_{e}\right) F_{i}\right]\right\}\right]\right|_{-\infty} ^{\infty}\right. \\
& \left.-\int_{-\infty}^{\infty} 2 v_{i}\left[\left\{\left[\frac{k T_{e}}{m_{i}}\right] \frac{\partial F_{i}}{\partial v_{i}}+\left[\left(v_{i}-\bar{V}_{e}\right) F_{i}\right]\right\}\right] d v_{i}\right\} .
\end{aligned}
$$


Since,

$$
\lim _{v_{i} \rightarrow \infty} \frac{\partial F_{i}}{\partial v_{i}}=\lim _{v_{i} \rightarrow-\infty} \frac{\partial F_{i}}{\partial v_{i}}=\lim _{v_{i} \rightarrow \infty} F_{i}=\lim _{v_{i} \rightarrow-\infty} F_{i}=0
$$

it follows that,

$$
\left.v_{i}^{2}\left[\left\{\left[\frac{k T_{e}}{m_{i}}\right] \frac{\partial F_{i}}{\partial v_{i}}+\left[\left(v_{i}-\vec{V}_{e}\right) F_{i}\right]\right\}\right]\right|_{-\infty} ^{\infty}=0
$$

Therefore,

$$
E Q I B=\left(\frac{m_{e} n_{e} \nu_{e i}}{2 n_{i}}\right)\left\{-\int_{-\infty}^{\infty} 2 v_{i}\left[\left\{\left[\frac{k T_{e}}{m_{i}}\right] \frac{\partial F_{i}}{\partial v_{i}}+\left[\left(v_{i}-\bar{V}_{e}\right) F_{i}\right]\right\}\right] d v_{i}\right\}
$$

Combining terms and splitting up the integral yields the following:

$$
E Q I B=\left(\frac{-m_{e} n_{e} \nu_{e i}}{n_{i}}\right)\left\{\left[\frac{k T_{e}}{m_{i}}\right] \int_{-\infty}^{\infty} v_{i} \frac{\partial F_{i}}{\partial v_{i}} d v_{i}+\int_{-\infty}^{\infty} v_{i}^{2} F_{i} d v_{i}-\bar{V}_{e} \int_{-\infty}^{\infty} v_{i} F_{i} d v_{i}\right\}
$$

Let,

$$
\begin{aligned}
I_{1} & =\int_{-\infty}^{\infty} v_{i} \frac{\partial F_{i}}{\partial v_{i}} d v_{i} \\
I_{2} & =\int_{-\infty}^{\infty} v_{i}^{2} F_{i} d v_{i} \\
I_{3} & =\int_{-\infty}^{\infty} v_{i} F_{i} d v_{i}
\end{aligned}
$$

then,

$$
E Q I B=\left(\frac{-m_{e} n_{e} \nu_{e i}}{n_{i}}\right)\left\{\left[\frac{k T_{e}}{m_{i}}\right] I_{1}+I_{2}-\bar{V}_{e} I_{3}\right\}
$$

Now consider the separate integral parts. First,

$$
I_{1}=\int_{-\infty}^{\infty} v_{i} \frac{\partial F_{i}}{\partial v_{i}} d v_{i}
$$


Integrating by parts,

$$
I_{1}=\left.\left[v_{i} F_{i}\right]\right|_{-\infty} ^{\infty}-\int_{-\infty}^{\infty} F_{i} d v_{i}
$$

From equation. 3.77 it can be seen that the first term is zero and from equation. 3.3 it follows that the second term is $n_{i}$. Therefore,

$$
I_{1}=-n_{i}
$$

Now consider $I_{3}$,

$$
I_{3}=\int_{-\infty}^{\infty} v_{i} F_{i} d v_{i}
$$

From equation. 3.4, one can clearly see that,

$$
I_{3}=n_{i} \bar{V}_{i}
$$

To solve for $I_{2}$, first consider the identity,

$$
\left(v_{i}-\bar{V}_{i}\right)^{2}=v_{i}^{2}-2 v_{i} \bar{V}_{i}+\bar{V}_{i}^{2} .
$$

Rearranging results in,

$$
v_{i}^{2}=\left(v_{i}-\bar{V}_{i}\right)^{2}+2 v_{i} \bar{V}_{i}-\bar{V}_{i}^{2} .
$$

Therefore, substituting into,

$$
I_{2}=\int_{-\infty}^{\infty} v_{i}^{2} F_{i} d v_{i}
$$


and splitting up the integrals yields,

$$
I_{2}=\int_{-\infty}^{\infty}\left(v_{i}-\bar{V}_{i}\right)^{2} F_{i} d v_{i}+2 \bar{V}_{i} \int_{-\infty}^{\infty} v_{i} F_{i} d v_{i}-\bar{V}_{i}^{2} \int_{-\infty}^{\infty} F_{i} d v_{i}
$$

From equation. 3.3 thru equation. 3.5, it is clear that,

$$
I_{2}=\frac{n_{i} k T_{i}}{m_{i}}+2 \bar{V}_{i} n_{i} \bar{V}_{i}-\bar{V}_{i}^{2} n_{i}
$$

Simplifying leaves one with,

$$
I_{2}=n_{i}\left[\frac{k T_{i}}{m_{i}}+\bar{V}_{i}^{2}\right]
$$

Substituting the integral values $\left(I_{1}, I_{2}\right.$, and $\left.I_{3}\right)$ into equation. 3.84 gives,

$$
E Q I B=\left(\frac{-m_{e} n_{e} \nu_{e i}}{n_{i}}\right)\left\{\left[\frac{k T_{e}}{m_{i}}\right]\left(-n_{i}\right)+n_{i}\left[\frac{k T_{i}}{m_{i}}+\bar{V}_{i}^{2}\right]-\bar{V}_{e} n_{i} \bar{V}_{i}\right\}
$$

Cancelling out an $n_{i}$ and combing terms,

$$
E Q I B=m_{e} n_{e} \nu_{e i}\left\{\frac{k}{m_{i}}\left[T_{e}-T_{i}\right]+\bar{V}_{i}\left[\bar{V}_{e}-\bar{V}_{i}\right]\right\}
$$

Now that the hard part is done, the derivation of the ion energy equation can continue.

The ion energy equation is obtained by taking the third moment of equation. 3.1 . If one sets $E=0$ and recalls that,

$$
\int_{-\infty}^{\infty} \frac{1}{2} m_{i} v_{i}^{2}\left[\left(\frac{\partial F}{\partial t}\right)_{i i}\right] d v_{i}=0
$$


since like particle collisions preserve energy. From the work above, one only needs to calculate,

$$
I_{4}=\int_{-\infty}^{\infty} \frac{1}{2} m_{i} v_{i}^{2}\left[\frac{\partial F_{i}}{\partial t}\right] d v_{i}
$$

From the derivation above, this is simply,

$$
I_{4}=\frac{m_{i}}{2} \frac{\partial}{\partial t} I_{2}
$$

Combining terms and recalling that $n_{i}$ is constant with respect to time gives,

$$
I_{4}=\frac{n_{i}}{2}\left[k \frac{\partial T_{i}}{\partial t}+m_{i} \frac{\partial V_{i}}{\partial t}\right]
$$

Therefore, the ion energy equation is,

$$
\frac{n_{i}}{2}\left[k \frac{\partial T_{i}}{\partial t}+m_{i} \frac{\partial V_{i}}{\partial t}\right]=m_{e} n_{e} \nu_{e i}\left\{\frac{k}{m_{i}}\left[T_{e}-T_{i}\right]+\bar{V}_{i}\left[\bar{V}_{e}-\bar{V}_{i}\right]\right\}
$$

For this test problem assume $\frac{\partial V_{i}}{\partial t}=\frac{\partial V_{e}}{\partial t}=V_{i}=V_{e}=0$. Then the ion energy equation becomes,

$$
\frac{n_{i} k}{2} \frac{\partial T_{i}}{\partial t}=\frac{m_{e} n_{e} \nu_{e i} k}{m_{i}}\left[T_{e}-T_{i}\right]
$$

Solving for $\frac{\partial T_{i}}{\partial t}$ yields,

$$
\frac{\partial T_{i}}{\partial t}=\frac{2 m_{e} n_{e} \nu_{e i}}{m_{i} n_{i}}\left[T_{e}-T_{i}\right]
$$

Similarly for electrons,

$$
\frac{\partial T_{e}}{\partial t}=\frac{-2 m_{e} n_{e} \nu_{e i}}{m_{i} n_{i}}\left[T_{e}-T_{i}\right]
$$


Notice that the RHS of equation. 3.105 is the negative of the RHS of equation. 3.104. This is because the energy removed from the ions must be added to the electrons to conserve energy and vice versa.

Using equation. 3.52 to combine equation. 3.104 and equation. 3.105 gives,

$$
\frac{\partial \Delta T}{\partial t}=\frac{-4 m_{e} n_{e} \nu_{e i}}{m_{i} n_{i}} \Delta T
$$

The solution to this is,

$$
\Delta T=\Delta T_{0} \exp (-k e q t)
$$

where,

$$
k e q=\frac{4 m_{e} n_{e} \nu_{e i}}{m_{i} n_{i}}
$$

\subsubsection{Results for Thermal Equilibrium Calculation}

Now that the exact solution has been derived, the thermal equilibrium problem is examined. This problem demonstrates the behavior of the numerical method on a problem which has widely varying time scales and four collision operators. Table 3.3 shows the input values used for this problem. Figure. 3.17 shows the non-dimensional temperature equilibrium rate for upwind differencing. This plot was generated from a set of four runs using different numbers of cells in the $v$ direction, $200,400,800$, and 1600 . All of the grids were accurate for the first $1.0 \times 10^{-5}$ seconds, but the 200 cell grid diverged from the other solutions after $2.0 \times 10^{-5}$. The other nodalizations effectively give the same answers and they are all close to the exact solution. Figure. 3.18 shows the transient values for the ion and electron temperatures for the 1600 cell case. From this plot, one can see that the electron temperature never quite reaches 


\begin{tabular}{|c|c|c|c|c|c|}
\hline \hline$n x$ & 1 & $n y$ & 200 & $T_{i 0}$ & $31.0 \mathrm{eV}$ \\
\hline$T_{e 0}$ & $29.0 \mathrm{eV}$ & $m_{i}$ & $2 \mathrm{AMU}$ & $m_{e}$ & $0.02 \mathrm{AMU}$ \\
\hline $\bar{V}_{i 0}$ & $0.0 \mathrm{~m} / \mathrm{s}$ & $\bar{V}_{e 0}$ & $0.0 \mathrm{~m} / \mathrm{s}$ & $n_{i 0}$ & $1.0 \times 10^{20} \mathrm{~m}^{-3}$ \\
\hline$n_{e 0}$ & $1.0 \times 10^{20} \mathrm{~m}^{-3}$ & $v_{i m a x}$ & $2.0 \times 10^{5} \mathrm{~m} / \mathrm{s}$ & $v_{e m a x}$ & $2.0 \times 10^{6} \mathrm{~m} / \mathrm{s}$ \\
\hline$\nu_{i i}$ & $2.5 \times 10^{4} \mathrm{~s}^{-1}$ & $\nu_{e e}$ & $4.0 \times 10^{6} \mathrm{~s}^{-1}$ & $\nu_{i e}$ & $4.0 \times 10^{4} \mathrm{~s}^{-1}$ \\
\hline$\nu_{e i}$ & $4.0 \times 10^{6} \mathrm{~s}^{-1}$ & tfinal & $200 \times \nu_{e e}^{-1}$ & $d t$ & $2.5 \times 10^{-7} \mathrm{~s}$ \\
\hline \hline
\end{tabular}

Table 3.3: Thermal Equilibrium Problem Input Values

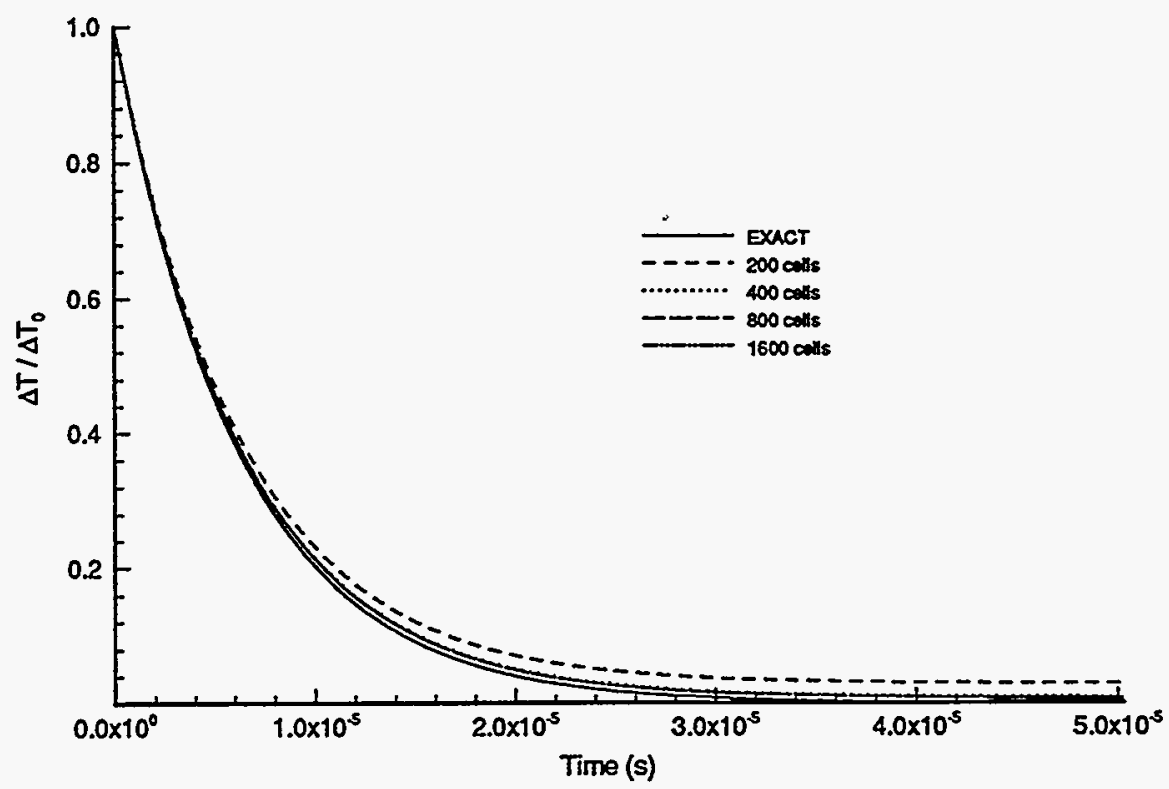

Figure 3.17: Upwind Equilibrium Calculations 


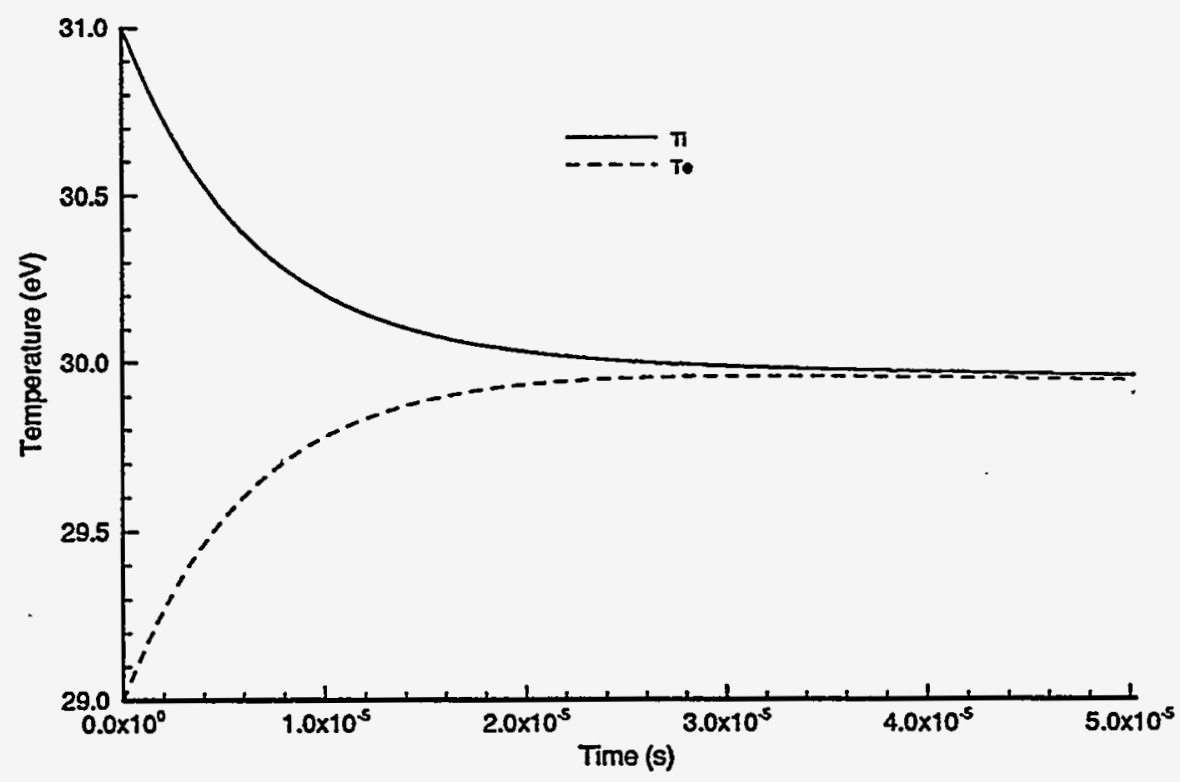

Figure 3.18: 1600 Cell Upwind Temperature Transient

the ion temperature. The two temperatures are about their closest at $3.2 \times 10^{-5}$ seconds when $T_{i}=29.98 \mathrm{eV}$ and $T_{e}=29.95 \mathrm{eV}$. Figure. 3.19 shows the temperature transient for upwind differencing. This simulation shows very similar behavior to that in figure. 3.10. Both ion and electron temperatures end up at about the same incorrect temperature of $85 \mathrm{eV}$, while the correct answer is $30 \mathrm{eV}$. The disturbing part of this simulation is the rate at which the incorrect answer was approached. In figure. 3.10 it took about $1.0 \times 10^{-3}$ seconds to reach 85 $\mathrm{eV}$, in figure. 3.19 the electrons reached $85 \mathrm{eV}$ in about $4.0 \times 10^{-6}$ second, about 250 times faster. The ions went to the wrong solution about ten times slower. This illustrates that the spurious transients are clearly a function of the collision frequency as shown in equation. 3.69.

In figure. 3.20, the dramatic improvement that the QUICK method has over upwind differencing can be seen. The temperatures stay within the correct boundaries and approach 


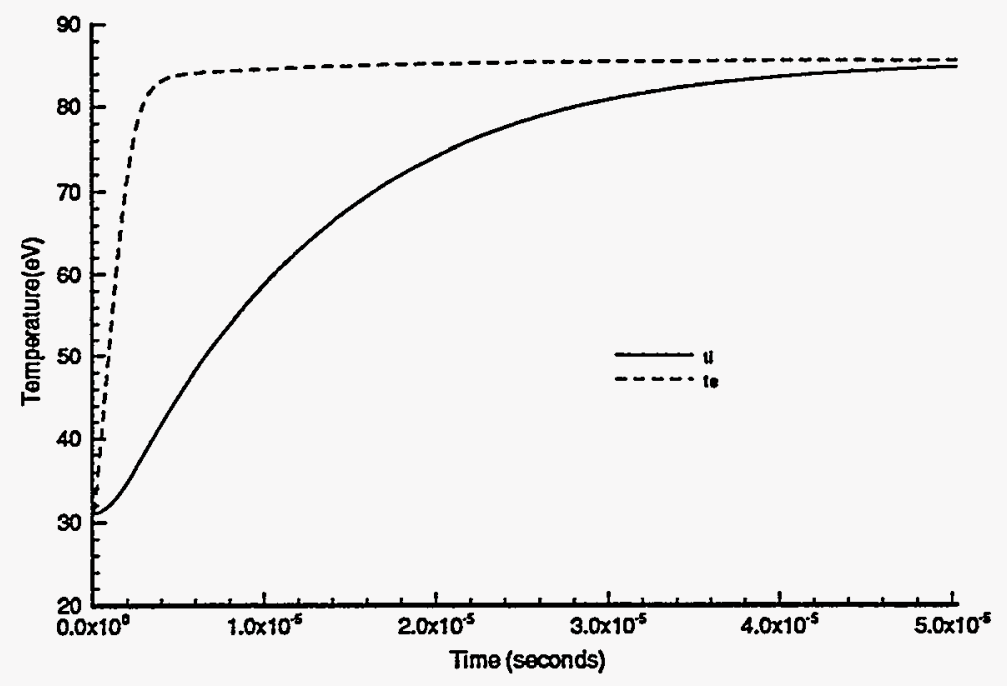

Figure 3.19: Upwind Differencing 200 cells

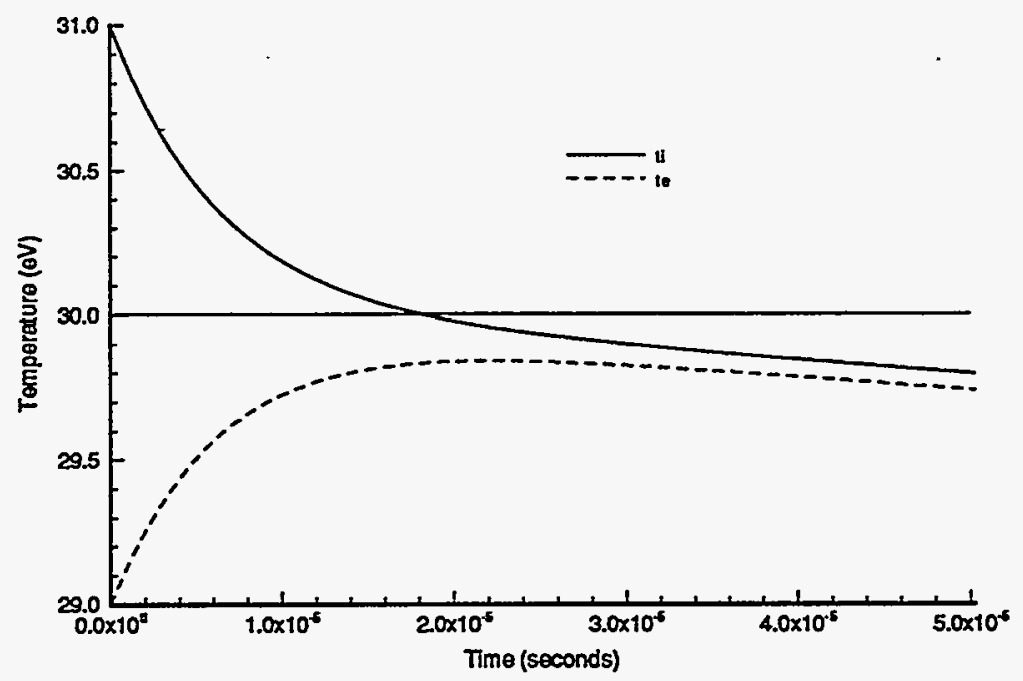

Figure 3.20: Quick Differencing 200 cells 
equilibrium. One can see however that there is still a significant temperature difference at the end of the transient and upon close observation, the cooling of both the ions and electrons can be noted.

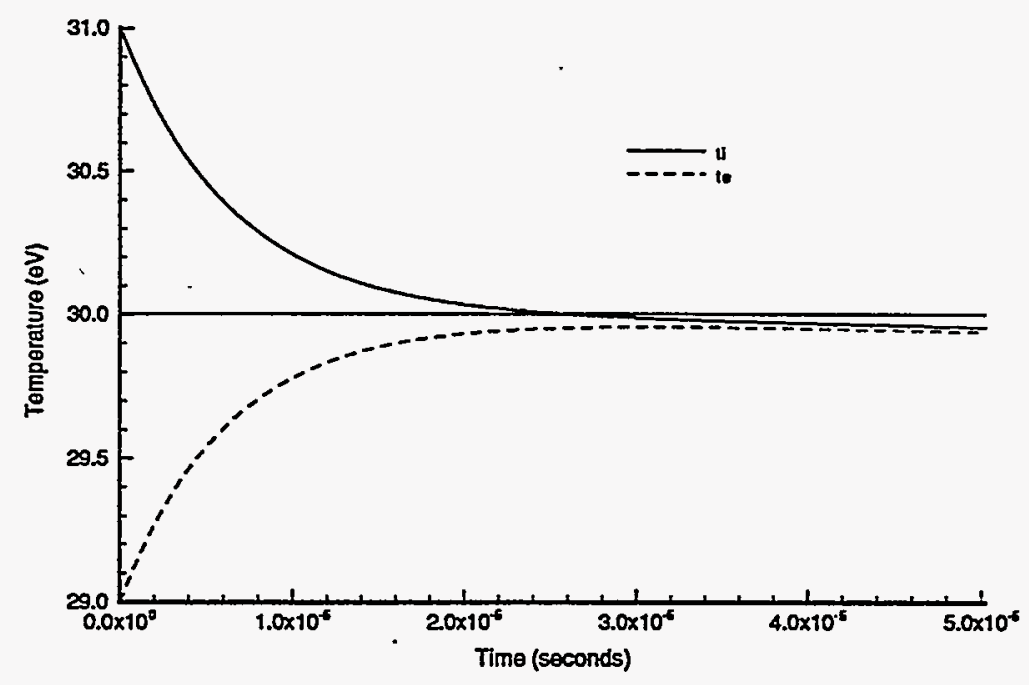

Figure 3.21: Chang-Cooper Interpolated Donor Cell Differencing 50 cells

Figure. 3.21 clearly shows that much better results can be obtained with a grid four times smaller. The advantage of the Chang-Cooper method over the QUICK method is actually even greater when one considers that the QUICK method uses a five point stencil while the Chang-Cooper method uses only a three point stencil.

\subsection{Summary}

If one is not careful in choosing a discretization scheme for the collision operators, artifcial transients, may overwhelm the simulation. This results from the accumulation of discretization error in the collision operators. It has been demonstrated that the Chang-Cooper method, which is designed specifically to eliminate this problem, outperforms other general 
higher order techniques like QUICK. 


\section{Chapter 4}

\section{Higher Order Differencing}

\subsection{Introduction}

There are two major obstacles to using a deterministic method for solving the VFP equation. The first is the large memory requirements for including the integral coupling of the electric field and the collision operators. The second is the difficulty in accurately discretizing the equations, so the correct physics is resolved. The large memory requirements of the long range coupling has been dealt- with in Chapter 2. The discretization problem has been partially dealt with in Chapter 3, where better differencing of the Folker-Plank collision operator was discussed. Therefore, the main obstacle left is the differencing of the Vlasov operator, and that is the topic of this chapter.

The rest of this chapter has the following organization. Section 4.2 presents a motivation for investigating Higher Order Differencing (HOD) of the first order terms. Section 4.3 presents the other differencing schemes that were investigated. Section 4.4 presents a numerical analysis problem, derives the exact solutions and presents the results. Section 4.5 then presents a calculation from Chapter 2 recomputed with the new HOD schemes and 
Section 4.6 summarizes the chapter.

\subsection{Motivation}

The simplest non-oscillatory first order differencing scheme for a convective derivative, $u \frac{\partial \phi}{\partial x}$, is the upwind differencing scheme [44]. Since the numerical method employed here is fully implicit, all differencing schemes are unconditionally stable, but depending on the differentiation scheme used, the solution may be oscillatory. The oscillatory solution, although it is stable, is clearly unphysical and should be eliminated. The central differencing method is more accurate than the first order upwind differencing, since it is second order from a Taylor series truncation analysis (see Appendix A), but it is also oscillatory. The non-oscillatory or monotonic behavior, of the upwind differencing scheme can be related to an "artificial viscosity" which is introduced by the method. Figure 4.1 presents the mesh values needed to

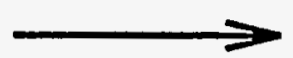

$\mathbf{u}_{\mathbf{i}}$

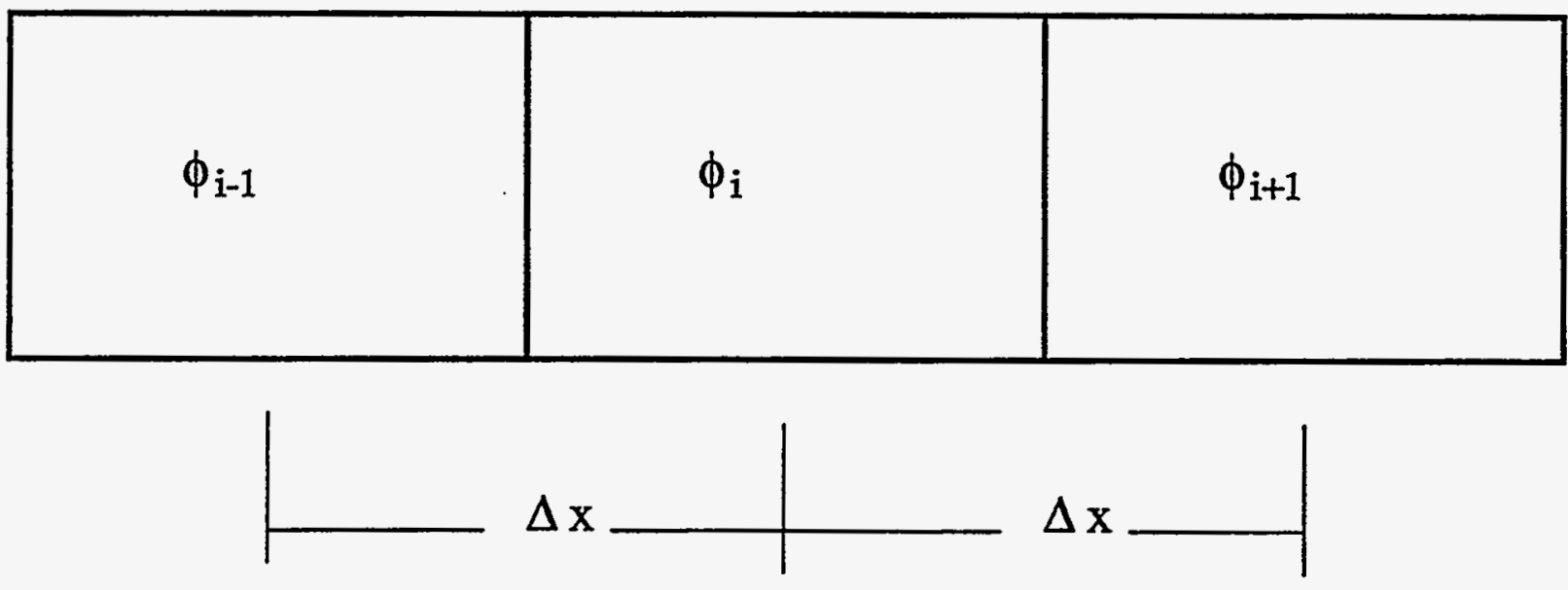

Figure 4.1: Upwind Discretization Mesh 
upwind difference the $\frac{\partial \phi}{\partial x}$ derivative with the vector $u$. The central difference approximation is,

$$
\left.u \frac{\partial \phi}{\partial x}\right|_{i C}=\frac{u_{i} \phi_{i+1}-u_{i} \phi_{i-1}}{2 \Delta x}
$$

Figure 4.1 shows that $u_{i}>0$, therefore the upwind differencing is,

$$
\left.u \frac{d \phi}{\partial x}\right|_{i U}=\frac{u_{i} \phi_{i}-u_{i} \phi_{i-1}}{\Delta x}
$$

Subtracting equation 4.2 from equation 4.1 yields

$$
\begin{aligned}
\left.u \frac{d \phi}{\partial x}\right|_{i C}-\left.u \frac{d \phi}{\partial x}\right|_{i U} & =\left(\frac{u_{i} \phi_{i+1}-u_{i} \phi_{i-1}}{2 \Delta x}\right)-\left(\frac{u_{i} \phi_{i}-u_{i} \phi_{i-1}}{\Delta x}\right) \\
& =\frac{u_{i} \phi_{i+1}-2 u_{i} \phi_{i}+u_{i} \phi_{i-1}}{2 \Delta x} \\
& =\left(\frac{u_{i} \Delta x}{2}\right)\left(\frac{\phi_{i+1}-2 \phi_{i}+\phi_{i-1}}{\Delta x^{2}}\right) \\
& \left.\approx\left(\frac{u_{i} \Delta x}{2}\right)\left(\frac{\partial^{2} \phi}{\partial x^{2}}\right)\right|_{i}
\end{aligned}
$$

Therefore, the following equation for the upwind differencing can be written,

$$
\left.\left.u \frac{d \phi}{\partial x}\right|_{i U} \approx u \frac{d \phi}{\partial x}\right|_{i C}-\left.\left(\frac{u_{i} \Delta x}{2}\right)\left(\frac{\partial^{2} \phi}{\partial x^{2}}\right)\right|_{i}
$$

Equation 4.3 demonstrates the numerical diffusion inherent in upwind differencing. This numerical diffusion is responsible for both the monotonicity and the inaccuracy of the upwind differencing method. The artificial viscosity, $\left.\left(\frac{\partial^{2} \phi}{\partial x^{2}}\right)\right|_{i}$, coefficient can be seen to be $\frac{u_{i} \Delta x}{2}$. The numerical diffusion in the upwind differencing method, therefore scales with $u_{i}$ and $\Delta x$. For the full 1D1V ion-electron problem, there are four terms in the Vlasov operator that are convective derivatives, $\left(v_{i} \frac{\partial F_{i}}{\partial x}, v_{e} \frac{\partial F_{e}}{\partial x}, \frac{q_{i} E}{m_{i}} \frac{\partial F_{i}}{\partial v_{i}}, \frac{q_{e} E}{m_{e}} \frac{\partial F_{e}}{\partial v_{e}}\right)$. From the above analysis, it is clear that 
the numerical diffusion in the Vlasov operator will therefore scale with, $v_{i}, v_{e}, \Delta v_{i}, \Delta v_{e}, \Delta x$, $\frac{q_{i} E}{m_{i}}$, and $\frac{q_{c} E}{m_{e}}$. A closer look at these values will help to illuminate the discretization problems inherent in the deterministic solution of the VFP equation.

First consider $v_{i}, v_{e}, \Delta v_{i}$, and $\Delta v_{e}$. The velocities have the range,

$$
\begin{aligned}
& -\left(v_{i}\right)_{\max } \leq v_{i} \leq\left(v_{i}\right)_{\max } \\
& -\left(v_{e}\right)_{\max } \leq v_{e} \leq\left(v_{e}\right)_{\max }
\end{aligned}
$$

where $\left(v_{i}\right)_{\max }$ and $\left(v_{e}\right)_{\max }$ range between two and seven times $\left(v_{i}\right)_{t h}$ and $\left(v_{e}\right)_{t h}$ respectively. The thermal velocities are related to the temperature through the following equations:

$$
\begin{aligned}
& \left(v_{i}\right)_{t h}=\left(\frac{2 k T_{i}}{m_{i}}\right)^{1 / 2} \\
& \left(v_{e}\right)_{t h}=\left(\frac{2 k T_{e}}{m_{e}}\right)^{1 / 2} .
\end{aligned}
$$

If the grid is uniform in velocity space then,

$$
\begin{aligned}
\Delta v_{i} & =\frac{\left(v_{i}\right)_{\max }-\left(-\left(v_{i}\right)_{\max }\right)}{n v} \\
\Delta v_{e} & =\frac{\left(v_{e}\right)_{\max }-\left(-\left(v_{e}\right)_{\max }\right)}{n v}
\end{aligned}
$$

where $n v$ is the number of cells in the $v$ direction.

There are a few trends that should be pointed out here. First, the boundaries of the velocity grid, where $v= \pm v_{\max }$, will have the highest levels of numerical diffusion. However, these velocity boundaries are where the number of particles is lowest. Recall that in a Maxwellian distribution the distribution function scales as $\exp \left[-(v)^{2}\right]$. Therefore, as long as 
$v_{\max }$ is chosen large enough, the numerical diffusion will not introduce a large error. However, since $\Delta v$ scales with $2 v_{\max }$, increasing $v_{\max }$ increases the error from $\Delta v$. Note however that $\Delta v$ can always be made smaller by increasing $n v$. Second, from observing the equation for $v_{t h}$ and assuming that $T_{i}=T_{e}$, it can be seen that

$$
\left(v_{e}\right)_{t h}=\left(v_{i}\right)_{t h}\left(\frac{m_{i}}{m_{e}}\right)^{1 / 2}
$$

Since $\Delta v$ and $v_{\max }$ scale with $v_{t h}$, the error in the electron grid, associated with $\Delta v$ and $v_{\max }$, will be $\left(\frac{m_{i}}{m_{e}}\right)^{1 / 2}$ times larger than the same error in the ion grid. Third, examining the $\frac{g E}{m_{c}} \frac{\partial F_{c}}{\partial v_{c}}$ terms and recalling that $q_{i}=-q_{e}$, it is noted that the error in the electron equation associated with this term will be $\left(\frac{m_{i}}{m_{e}}\right)^{3 / 2}$ times larger than the same error in the ion equation. It should now be clear that the main discretization problem will be in the electron equation where the error scales for a hydrogen plasma as $\left(\frac{m_{i}}{m_{e}}\right)^{3 / 2}$ times larger than the ion equation. Recall that the mass ratio is, $\frac{m_{i}}{m_{e}} \approx 1800$, although simulations may be done with a reduced mass ratio of $\approx 100$.

Finally, there is the $\Delta x$ error, but $\Delta x$ is set by the problem geometry and $n x$, where $n x$ is the number of cells in the $x$ direction. Thus, this error can be minimized by a finer discretization of the grid.

\subsection{Numerical Schemes}

This section will discuss the numerical schemes that can be employed to evaluate the discrete first order differential operator. In the previous section, numerical diffusion is pointed out as a possible source of error when upwind differencing is used. The numerical diffusion also has 
a monotonic preserving effect on the solution. This section will now present two methods, one very primitive and one much more advanced, for keeping the discretization scheme monotonic while also minimizing numerical diffusion.

\subsubsection{Interpolated Donor Cell (IDC) Differencing}

The simplest differencing scheme beyond upwind differencing is interpolated donor cell differencing. From equation 4.3 it can be seen that upwind differencing is approximately central differencing with an added numerical viscosity. It is known that central differencing has truncation error of order $(\Delta x)^{2}, O\left(\Delta x^{2}\right)$, but it can be oscillatory. Upwind differencing, which is $O(\Delta x)$, is monotonic. Therefore, a simple approach to obtain a monotonic method with less numerical diffusion would be to average upwind differencing with central differencing. Note, that from a Taylor series expansion approach, IDC is only first order accurate. The equation for this approach follows:

$$
\left.u \frac{d \phi}{\partial x}\right|_{i I D C}=\left.w u \frac{d \phi}{\partial x}\right|_{i U}+\left.(1-w) u \frac{d \phi}{\partial x}\right|_{i C}
$$

where $w$ is the weighting parameter used to average the two schemes. If equation 4.3 is combined with equation 4.5 it yields

$$
\left.u \frac{d \phi}{\partial x}\right|_{i I D C}=\left.u \frac{d \phi}{\partial x}\right|_{i C}+\left.w\left(\frac{u_{i} \Delta x}{2}\right)\left(\frac{\partial^{2} \phi}{\partial x^{2}}\right)\right|_{i}
$$

The numerical viscosity coefficient in this formulation is $w\left(\frac{u_{i} \Delta x}{2}\right)$. The goal now becomes to choose the smallest value of $w$ that will make the central differencing scheme monotonic. Clearly from equation 4.6 , the smaller the value chosen for $w$, the smaller the amount of 
numerical diffusion.

\subsubsection{Non-Linear Differencing (Flux Limiting)}

First note that in this chapter and in the following chapters, that flux limiting is a discretization technique and not the transport equation restriction discussed in Chapter 1 as "flux limiting".

The basic idea behind flux limiting is to modify higher order methods, which are known to have oscillation problems, so they remain monotonic. This assured monotonicity prevents the higher order methods from introducing false local minima or maxima that are caused by their oscillatory nature. The non-linearity comes from the dependence of the differencing on the value that is being differenced. Therefor, as the solution changes, the form of the discretization changes with it.

Flux-Corrected Transport (FCT) [69] was an early explicit non-linear, monotone, scheme applied to the Vlasov operator. Since this method was two-step in nature, it is only easily applied to explicit differencing. Recently, there has been much progress made in implicit nonlinear, monotone, convection schemes in computational fluid dynamics. The methodology developed for implicit differencing in computational fluid dynamics will be employed in this dissertation for the implicit solution of the Vlasov-Fokker-Planck equation.

To illuminate the oscillatory behavior of higher order schemes in general and QUICK (Quadratic Upstream Interpolation for Convective Kinematics, Section 3.4.1) in particular, a simple demonstration will be presented which shows how flux limiting improves higher order differencing schemes by making them monotonic. The function that will be used in this 
demonstration is a Maxwellian distribution,

$$
F_{m}=n_{0}\left(\frac{1}{v_{t h}^{2} \pi}\right)^{1 / 2} \exp \left(\frac{-\left(v_{l o c}-\bar{V}_{0}\right)^{2}}{v_{t h}^{2}}\right)
$$

where in discrete form $v_{l o c}=j \Delta v-v_{\max }$ with $\Delta v=\frac{2 v_{\max }}{n v}$. The numerical values used to make figure 4.2 are shown in Table 4.1 .

\begin{tabular}{|c|c|c|c|c|c|}
\hline \hline$n v$ & 100 & $v_{t h}$ & $53800 \mathrm{~m} / \mathrm{s}$ & $T_{0}$ & $30.0 \mathrm{eV}$ \\
\hline$V_{0}$ & $1.0 \times 10^{5} \mathrm{~m} / \mathrm{s}$ & $v_{\max }$ & $10 v_{t h}$ & $n_{0}$ & $1.0 \times 10^{20} \mathrm{~m}^{-3}$ \\
\hline$m$ & $2 A M U$ & $f l_{c}$ & 2 & $v_{l o c}$ & $10 v_{t h}$ \\
\hline \hline
\end{tabular}

Table 4.1: Flux Limit Example Input Values

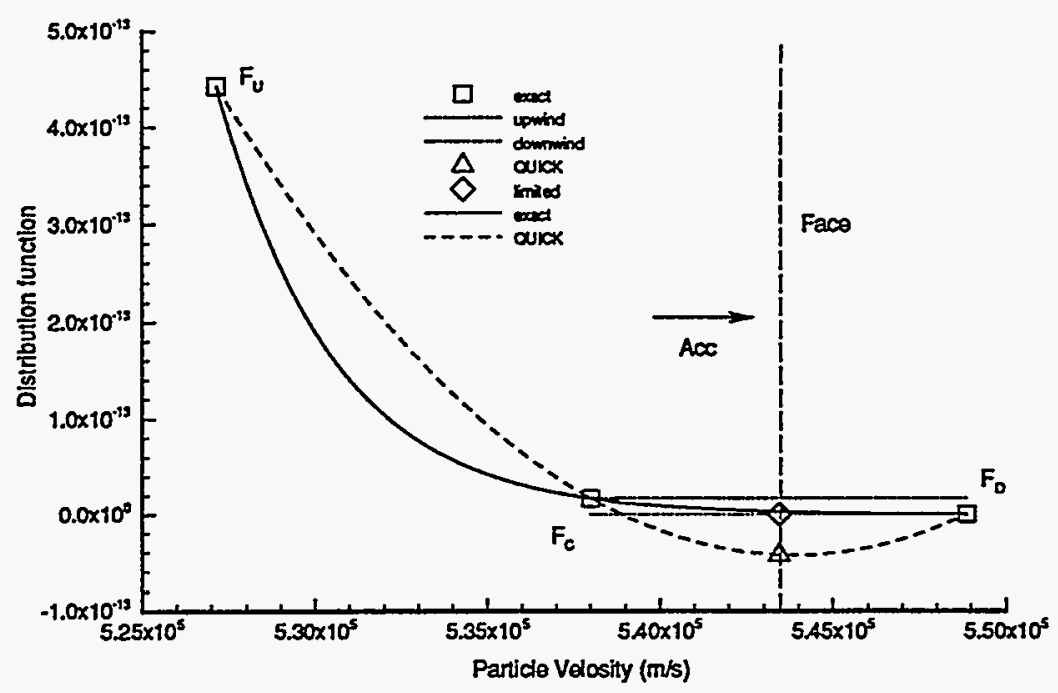

Figure 4.2: Flux Limiting. Example

Figure 4.2 shows equation 4.7 plotted over the range $\left(v_{l o c}+\Delta v, v_{l o c}-\Delta v\right)$. This figure shows how the term $A c c \frac{\partial F}{\partial v}\left(A c c=\frac{q E}{m}\right)$ would be evaluated in a finite volume discretization. The squares on the plot show the value of $F$ at three cell centers, the upwind cell $F_{U}$, the 
center cell $F_{C}$, and the downwind cell $F_{D}$. The solid line joining them represents the exact value of the Maxwellian curve. The goal of the QUICK method is to provide a value of $F$ evaluated at the cell face, $F_{f}$, where the cell face is represented by the vertical dashed line. The other dashed line shows the quadratic curve placed through the three cell values by the QUICK method. The triangle, therefore shows the QUICK interpolated value at the cell face. Thus, the QUICK method has given a face value that is lower than both $F_{C}$ and $F_{D}$. Moreover, the QUICK method has given a negative value for the distribution function at the cell face, which is clearly an unphysical, unacceptable result. Figure 4.2 clearly shows how higher order methods can introduce false local minima. The dotted lines, upwind and downwind, show the maximum and the minimum values that the face value could take and still preserve monotonicity, (i.e., no new local minima or maxima). Since the higher order QUICK method has a value outside of the monotone preserving region, the flux limiting algorithm chooses the downwind boundary line (the diamond) which is the closest to the higher order method. Now that this example has been examined, the details of the flux limiting algorithm will be presented.

In the early work of Boris and Book [69], Flux-Corrected Transport (FCT) was accomplished in a two step process. The first step was highly diffusive, such as upwind differencing, and then the second step was anti-diffusive to try to remove as much of the diffusion as possible while maintaining monotonicity. This method, because of its two step process, lends itself better to explicit differencing rather than the fully implicit differencing used in this work.

Gaskell and Lau [71], who where working on solving the incompressible Navier-Stokes equations, have developed a method to ensure monotonicity of the variables which is expressed 
in terms of limits on non-dimensional quantities. They have defined the non-dimensionalization by,

$$
\tilde{F}=\frac{F-F_{U}}{F_{D}-F_{U}} .
$$

where $\tilde{F}$ is the non-dimensional value of $F, F_{U}$ is the upwind cell value, and $F_{D}$ is the downwind cell value defined the same as in figure 4.2. Using this non-dimensionalization, Gaskell and Lau determined a monotonic conserving relationship between the non-dimensional face value, $\tilde{F}_{f}$, and the non-dimensional center grid value, $\tilde{F}_{C}$. This relationship can be described by figure 4.3. Gaskell and Lau's Convection Boundedness Criterion (CBC), which preserves

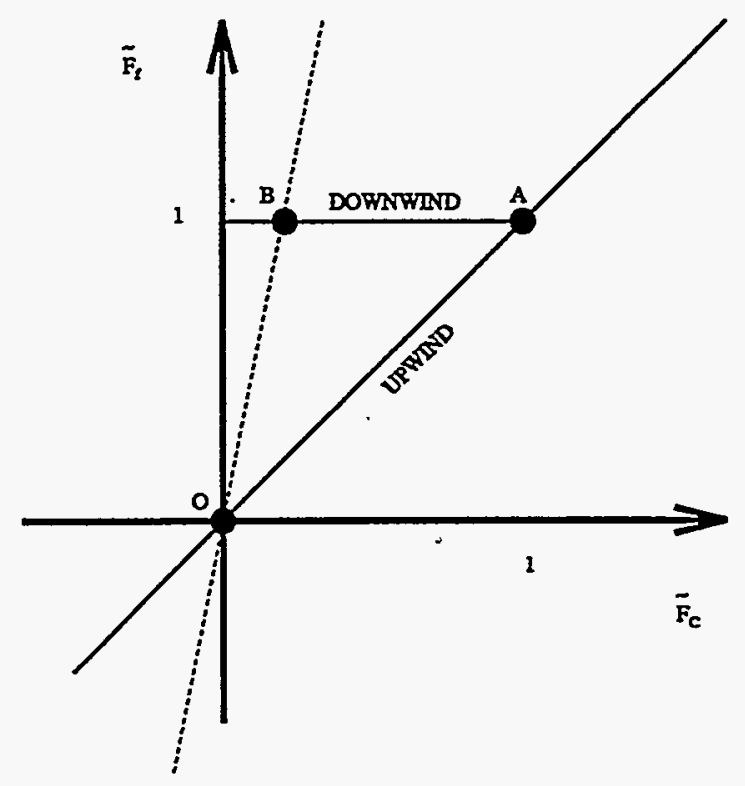

Figure 4.3: Non-Dimensional Flux Limiting Criteria

monotonicity, is met anywhere inside of the triangle $(0,0),(0,1),(1,1)$ and anywhere on the line $\tilde{F}_{f}=\tilde{F}_{C}$, which defines upwind differencing. From equation 4.8 recall that

$$
\tilde{F}_{f}=\frac{F_{f}-F_{U}}{F_{D}-F_{U}}
$$




$$
\tilde{F_{C}}=\frac{F_{C}-F_{U}}{F_{D}-F_{U}}
$$

To fully understand figure 4.3, some of the special cases that can occur, as shown in Table 4.2, need to be considered. Considering figure 4.2 and 4.3 and Table 4.2 , one can discuss the six

\begin{tabular}{|c|c|c|c|}
\hline \hline CASE & Dimensional Condition & Non-dimensional result & Comment \\
\hline \hline 1 & $F_{f}=F_{C}$ & $\tilde{F}_{f}=\tilde{F}_{C}$ & Upwind \\
\hline 2 & $F_{f}=F_{D}$ & $\tilde{F}_{f}=1$ & Downwind \\
\hline 3 & $F_{f}=F_{C}=F_{D}$ & $\tilde{F}_{f}=\tilde{F}_{C}=1$ & A \\
\hline 4 & $F_{f}=F_{U}$ & $\tilde{F}_{f}=0$ & $\tilde{F}_{C}$ axis \\
\hline 5 & $F_{C}=F_{U}$ & $\tilde{F}_{C}=0$ & $\tilde{F}_{f}$ axis \\
\hline 6 & $F_{U}=F_{D}$ & $\tilde{F}_{f}=\tilde{F}_{C}=\infty$ & Breakdown \\
\hline
\end{tabular}

Table 4.2: Flux Limiting Special Cases

special cases:

1. This is simply upwind differencing. Figure 4.3 shows that the 45 degree line of the triangle, line $\mathrm{OA}$, is the highly diffusive and very stable upwind differencing.

2. This is the top of the triangle which corresponds to downwind differencing.

3. This is point $A$ in figure 4.3. It should be noted from Figure 4.2, that this corresponds to the downwind half of the stencil being completely flat (i.e., $\left.\frac{d F}{\partial v}\right|_{d o w n}=0$ ).

4. This case defines the $\tilde{F}_{C}$ axis in figure 4.3 .

5. For this case the value of $\tilde{F}_{f}$ is not well defined (i.e., it could be any value along the axis between zero and one). To remove this uncertainty in $\tilde{F}_{f}$ the line OB is added to the graph. This line is defined by,

$$
\tilde{F}_{f}=f l_{c} \tilde{F}_{C}
$$


where $f l_{c}$ is the slope of the line. This can be converted to dimension variables using equation 4.8 by the following,

$$
\begin{aligned}
\frac{F_{f}-F_{U}}{F_{D}-F_{U}} & =f l_{c} \frac{F_{C}-F_{U}}{F_{D}-F_{U}}, \text { or } \\
F_{f} & =F_{U}+f l_{c}\left(F_{C}-F_{U}\right)
\end{aligned}
$$

Figure 4.4 displays an example of when equation 4.10 is used. Note all definitions in

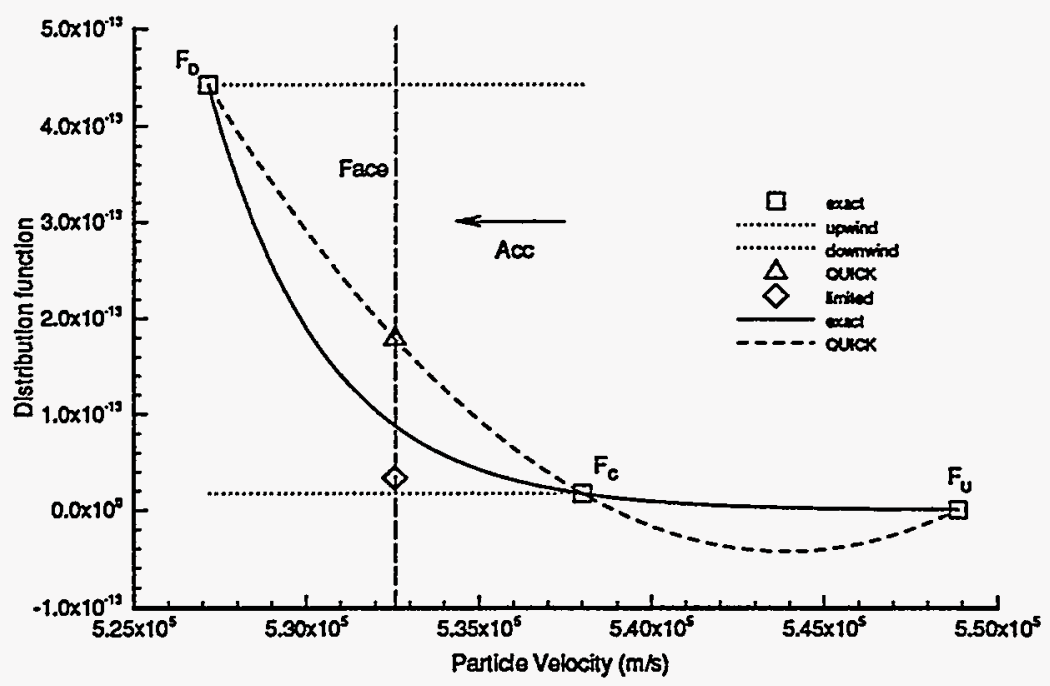

Figure 4.4: Flux Limiting Example 2

the pictures are the same as in figure 4.2 , but the acceleration force has been reversed. Here the monotonicity of the solution is easily maintained because of the large difference between $F_{C}$ and $F_{D}$. The higher order QUICK method also produces a monotonic value, the triangle. But this time the higher order result is in the $O,(0,1), B$ triangle of figure 4.3 , not the accepted $O, B, A$ triangle. The flux limited value, the diamond, is determined by equation 4.10 . The height up the face towards the HOD method, or 
triangle, is determined by the value of $f l_{c}$. Table 4.1 shows that the value chosen was $f l_{c}=2$. As the value of $f l_{c}$ is raised the flux limited value will increase until it reaches the HOD value. From figure 4.4 it is clear that the accuracy of the flux limited method will increase as the value of $f l_{c}$ is increased until the value reaches the exact solution. At that point, increasing $f l_{c}$ will decrease the accuracy. Therefore, unless one knows the shape of the curve (i.e., a Maxwellian) the choice of $f l_{c}$ is somewhat arbitrary.

6. This is where the numerical implementation breaks down, and this must be removed from the limiter. Since the non-dimensionalization results in a divide by zero, the values of $\tilde{F}_{f}$ and $\tilde{F}_{C}$ go to infinity and the numerical simulation will fail.

In 1995, Leonard and Drummond presented a flux limiting scheme in terms of the dimensional variables and a median function, $M E D(a, b, c)$. Here the median function returns one of the three variables, $(a, b, c)$, whose value lies between the other two variables. Let $X_{h}=\max (a, b, c)$, and let $X_{l}=\min (a, b, c)$, then

$$
\operatorname{MED}(a, b, c)=a+b+c-X_{h}-X_{l}
$$

This implementation is as follows,

$$
\begin{aligned}
& F_{1}=F_{C} \\
& F_{2}=F_{D} \\
& F_{3}=F_{U}+f l_{c}\left(F_{C}-F_{U}\right) \\
& F_{4}=M E D\left(F_{1}, F_{2}, F_{3}\right) \\
& F_{f}=M E D\left(F_{1}, F_{4}, F_{f}^{H O D}\right)
\end{aligned}
$$


where $F_{f}^{H O D}$ is the face value from a Higher Order Differencing (HOD) scheme such as QUICK.

The equation, $F_{1}=F_{C}$, defines the hypotenuse of the triangle, as can be seen from figure 4.3 and Table 4.2. Similarly, one can see that $F_{2}=F_{D}$ gives the top horizontal line of the triangle. Equation 4.10 defines the dotted line in figure 4.3 that passes through the points $O$ and $\mathrm{B}$. Now from figure 4.3, it is clear that the line $\overline{O A}$ is always below the dotted line and the top of the triangle, therfore $F_{4}=\operatorname{MED}\left(F_{1}, F_{2}, F_{3}\right)$ defines the discontinuous line segment $\overline{O B A}$. Finally, the $F_{f}=M E D\left(F_{1}, F_{4}, F_{f}^{H O D}\right)$, step determines if the higher order method is inside of the triangle. If so, it is used, otherwise the boundary of the triangle which is closest to $F_{f}^{H O D}$ is used.

The first step in the continued evolution of the flux limiting method is to remove the downwind line (BA in figure 4.3). This is done to remove the degenerate case that can occur if the solution encounters a sharp gradient along the diagonal of the cell. Figure 4.5 shows

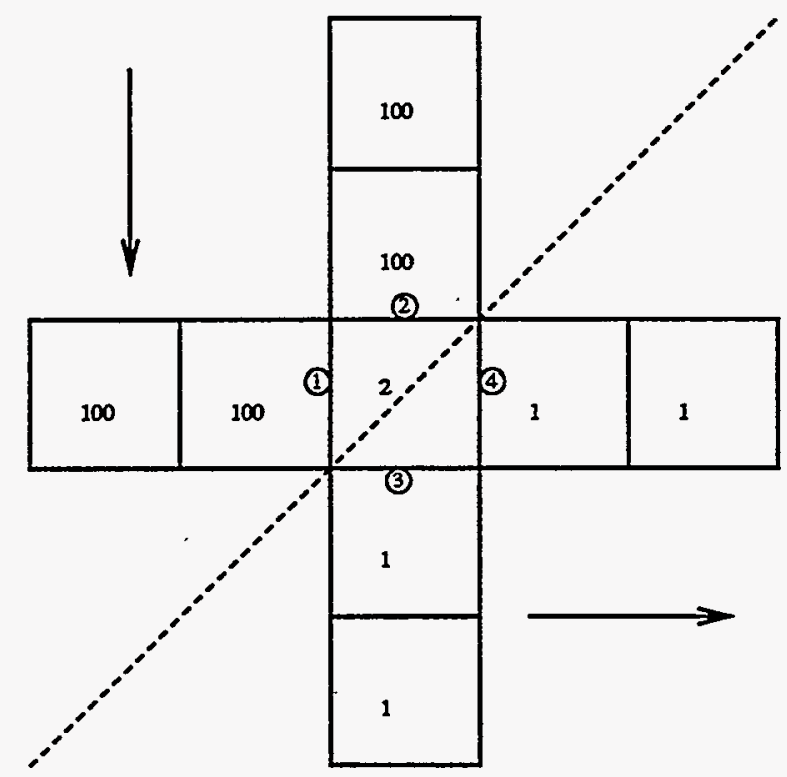

Figure 4.5: Degenerate Case of a Diagonal Discontinuity 
a stencil for a cell that has a sharp gradient along its diagonal. From the arrows indicating flow direction, it is clear that the values for face 1 and 2 will be equal and the values for face 3 and 4 will be equal. First consider faces 1 and 2 and the flux limiting algorithm.

$$
\begin{aligned}
& F_{U}=100 \\
& F_{C}=100 \\
& F_{D}=2
\end{aligned}
$$

$$
\begin{aligned}
& F_{1}=F_{C}=100 \\
& F_{2}=F_{D}=2 \\
& F_{3}=F_{U}+f l_{c}\left(F_{C}-F_{U}\right)=100+2(100-100)=100 \\
& F_{4}=M E D\left(F_{1}, F_{2}, F_{3}\right)=100=\text { upwind } \\
& F_{f}=M E D\left(F_{1}, F_{4}, F_{f}^{H O D}\right)=100=\text { upwind, }
\end{aligned}
$$

The last line is true because $F_{1}=F_{4}$. Therefore, upwind differencing will be used for faces 1 and 2. Now consider the non-dimensional numbers,

$$
\begin{aligned}
& \tilde{F}_{f}=\frac{F_{f}-F_{U}}{F_{D}-F_{U}}=\frac{100-100}{2-100}=0 \\
& \tilde{F}_{C}=\frac{F_{C}-F_{U}}{F_{D}-F_{U}}=\frac{100-100}{2-100}=0 .
\end{aligned}
$$

These non-dimensional numbers show that the values are at point $O$ in Fig 4.3. Now consider 
faces 3 and 4.

$$
\begin{aligned}
& F_{U}=100 \\
& F_{C}=2 \\
& F_{D}=1
\end{aligned}
$$

$$
\begin{aligned}
& F_{1}=F_{C}=2 \\
& F_{2}=F_{D}=1 \\
& F_{3}=F_{U}+f l_{c}\left(F_{C}-F_{U}\right)=100+2(2-100)=-96 \\
& F_{4}=M E D\left(F_{1}, F_{2}, F_{3}\right)=1=\text { downwind } \\
& F_{f}=M E D\left(F_{1}, F_{4}, F_{f}^{H O D}\right)=1=\text { downwind, }
\end{aligned}
$$

The last line is true because $F_{f}^{H O D}<1$. Now consider the non-dimensional numbers.

$$
\begin{aligned}
& \tilde{F}_{f}=\frac{F_{f}-F_{U}}{F_{D}-F_{U}}=\frac{1-100}{1-100}=1 \\
& \tilde{F}_{C}=\frac{F_{C}-F_{U}}{F_{D}-F_{U}}=\frac{2-100}{1-100}=\frac{98}{99} .
\end{aligned}
$$

These non-dimensional numbers show that these values are near point $A$ in Fig 4.3 on the downwind differencing line. It is now clear that faces 3 and 4 will be downwind differenced. But, if faces 1 and 2 are upwind differenced and faces 3 and 4 are downwind differenced, then the cell in the middle of the stencil will not enter into the matrix because it does not effect any other cells including itself. Therefore, the matrix will be singular. To eliminate the matrix singularity a slanted line will be used to replace the downwind line. This is shown as 
the dotted line $B A$ in figure 4.6. With this new dotted line the limiter algorithm becomes,

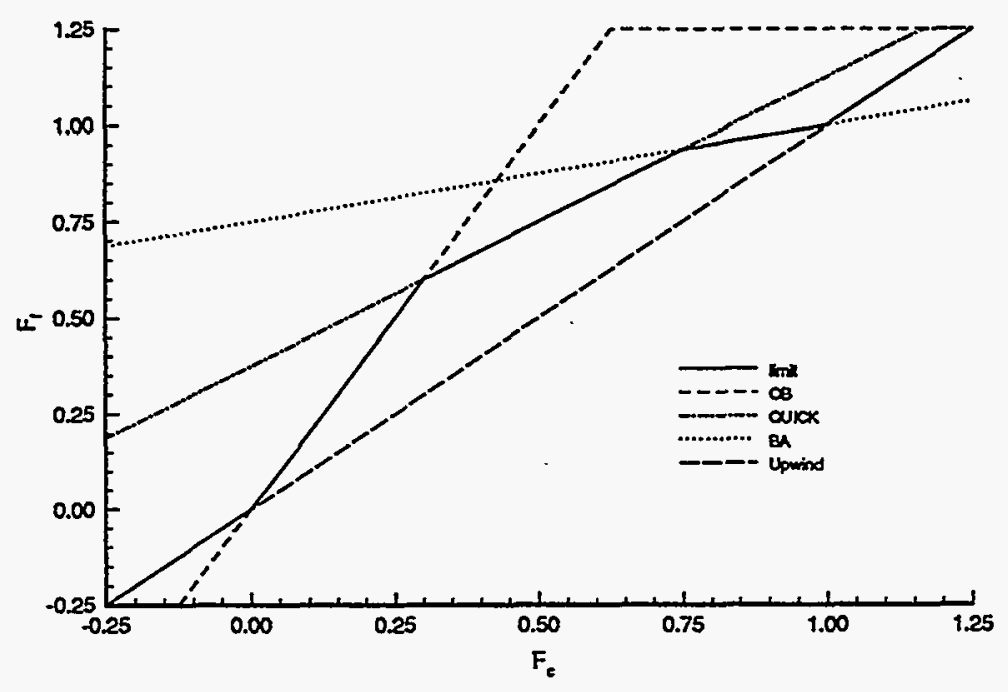

Figure 4.6: Components of the Flux Limiter

$$
\begin{aligned}
& F_{1}=F_{C} \\
& F_{2}=\left(\beta F_{C}\right)+(1-\beta) F_{D} \\
& F_{3}=F_{U}+f l_{c}\left(F_{C}-F_{U}\right) \\
& F_{4}=M E D\left(F_{1}, F_{2}, F_{3}\right) \\
& F_{f}=M E D\left(F_{1}, F_{4}, F_{f}^{H O D}\right) .
\end{aligned}
$$

If the extensions are removed, then the result is the flux limiter defined by figure 4.7. For figure $4.7 \beta=.25$ and $f l_{c}=2$. Upon close observation of figure 4.7 , one notices that there are sharp corners at points $1,2,3$, and 4 . It has been found that sometimes the Newton iteration (i.e., the non-linear iteration defined in Chapter 2) will have difficulty converging with this limiter. For example, one will see a particular cell preventing convergence because it 


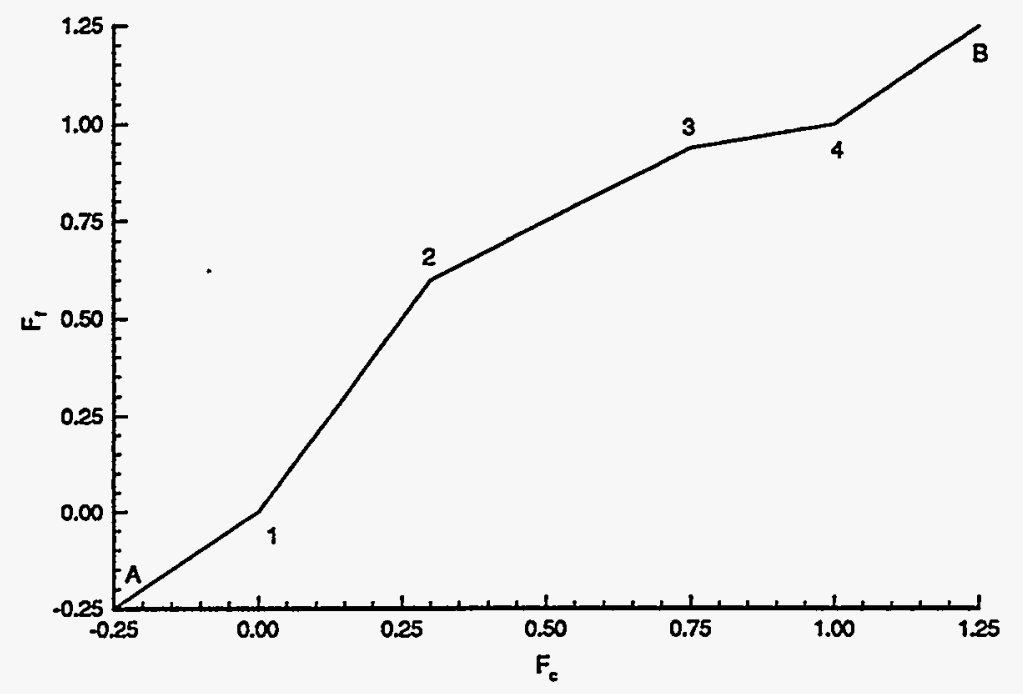

Figure 4.7: Non-Differentiable (Rough) Flux Limiter

is oscillating between two points near 3 , but one is on the $3-4$ line and the other is on the $2-3$ line. Evidence of this stalled convergence behavior can be seen in figure 4.9 and figure 4.10 in the dotted "rough" lines.

These two figures are computed from a double Maxwellian test problem (see Table 4.6 and figure 4.17 of Section 4.4.2). This problem is run on a $100 \times 100$ grid and upwind differencing is used for the Vlasov operator. The results in figure 4.9 are generated by using the steady-state results for upwind differencing as an initial guess for the flux-limited QUICK differencing and running the problem to steady state using the steady-state method of solution. The results in figure 4.10 are computed the same way but the pseudo-transient option is used instead.

Because of the stalling behavior, the limiter was modified to make it differentiable or "smooth". The new smooth limiter can be seen in figure 4.8. For a complete description of the implementation of the differentiable limiter see Appendix B. Figure 4.9 and figure 4.10 show the effect that the new "smooth" limiter has on eliminating the convergence stall of the 
"rough" limiter for both steady state and transient runs.

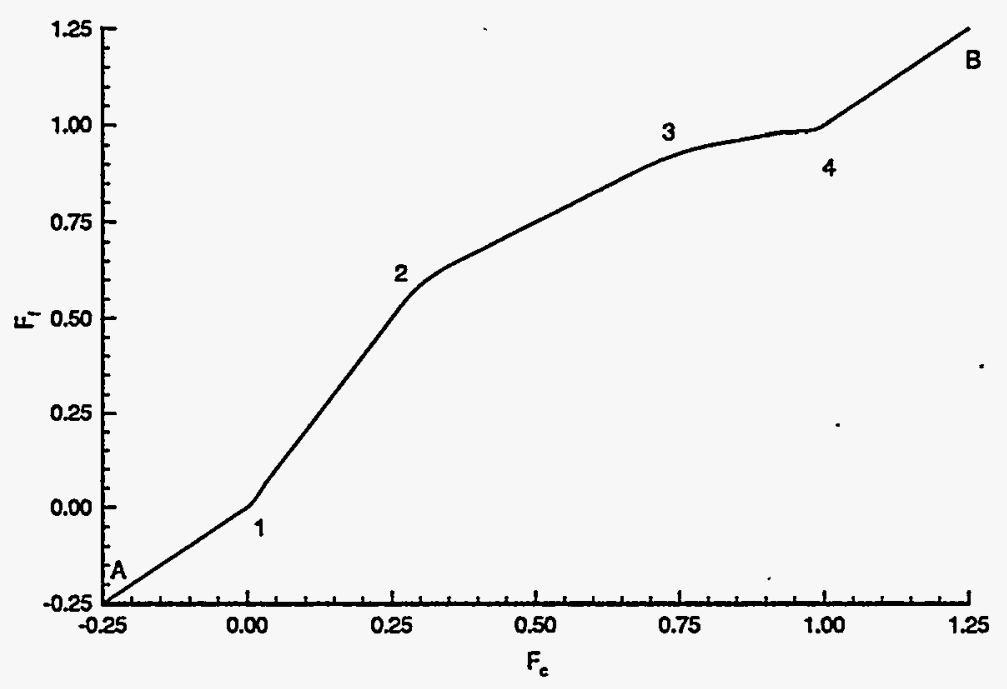

Figure 4.8: Differentiable (Smooth) Flux Limiter

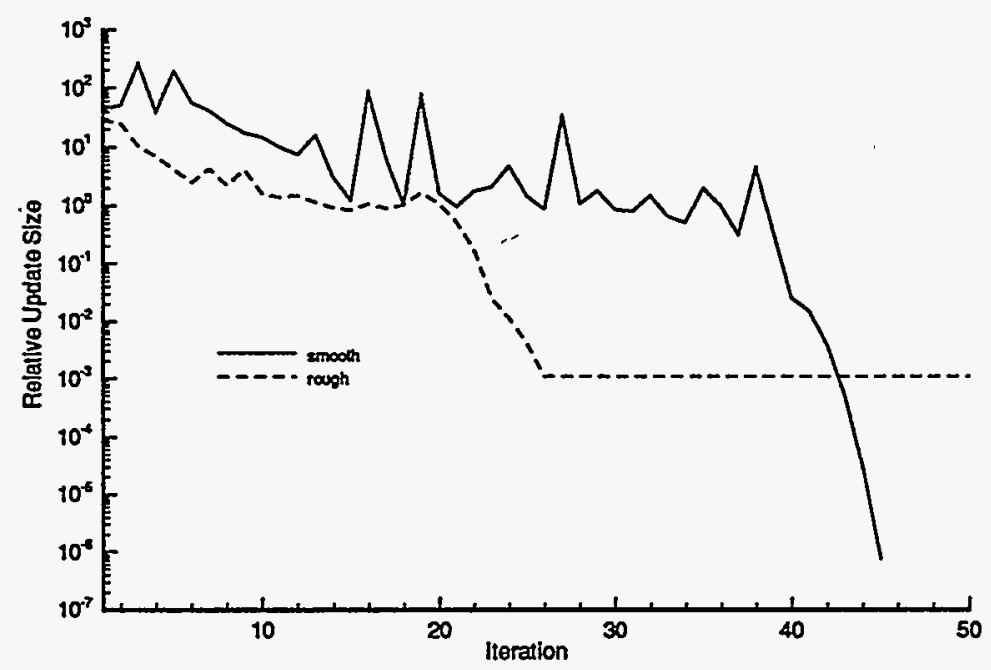

Figure 4.9: Differentiable vs. Non-Differentiable Limiter Convergence for a Steady State Run 


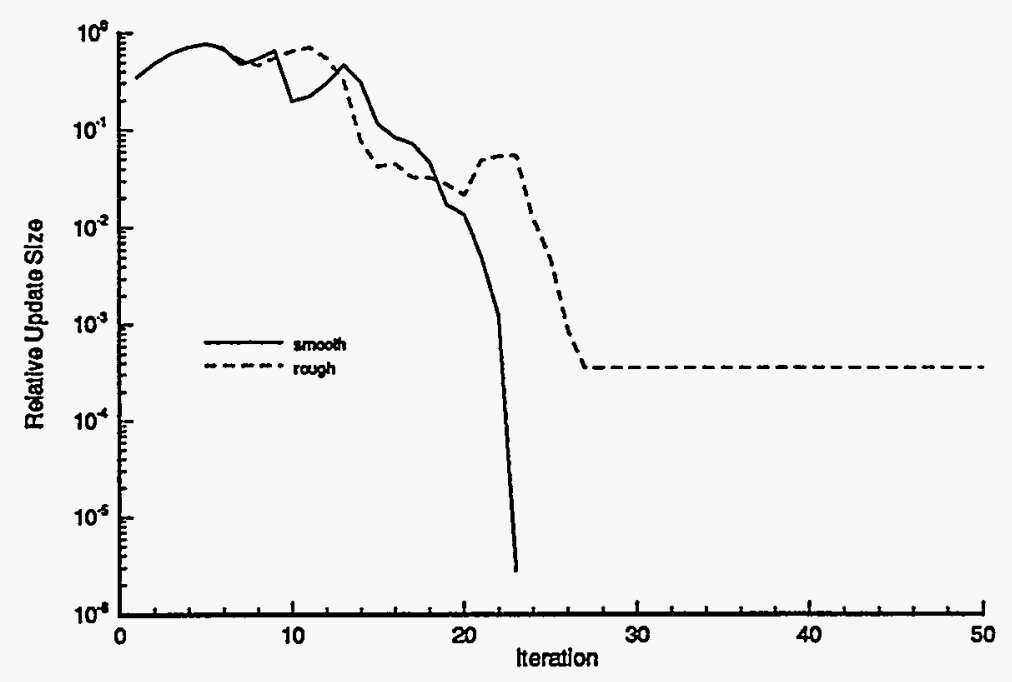

Figure 4.10: Differentiable vs. Non-Differentiable Limiter Convergence for a Transient Run

\subsection{Mathematical Model}

Consider a model Vlasov-like operator in general mathematical terms as in the following equation,

$$
y \frac{\partial z}{\partial x}+A(x) \frac{\partial z}{\partial y}=0
$$

For simple forms of $A(x)$, this equation will have exact solutions. One can therefore compute a numerical solution and compare it to the exact solution to determine the error in the discretization scheme associated with the first order operators. The exact solution will be described in Subsection 4.4.1 and the numerical calculations for comparison will be shown in Section 4.4.2. 


\subsubsection{Exact Solution}

First consider $A(x)=A_{0}$. One can then solve equation 4.12 by the method of characteristics [72]. Consider a problem restricted to the upper half plane where the particle velocity is positive. The boundary condition can be described by,

$$
z(0, y)=Z(y)
$$

Dividing equation 4.12 by $y$ results in,

$$
\frac{\partial z}{\partial x}+\frac{A_{0}}{y} \frac{\partial z}{\partial y}=0
$$

From the product rule of differentiation one gets,

$$
y d y=\frac{1}{2} d\left(y^{2}\right)
$$

Using this identity results in,

$$
\frac{\partial z}{\partial x}+2 A_{0} \frac{\partial z}{\partial y^{2}}=0
$$

This problem, equation 4.16 with equation 4.13 for boundary conditions, is solved in Table 4.3.

From the last row of column two of Table 4.3, $t$ can be eliminated yielding,

$$
t=x
$$




\begin{tabular}{|c|c|c|c|c|cc|}
\hline Characteristics & $\frac{d x}{d t}=1$ & $\frac{d y^{2}}{d t}=2 A_{0}$ & $\frac{d z}{d t}=0$ \\
\hline Solution of Characteristics & $x=t+c_{1}$ & $y^{2}=2 A_{0} t+c_{2}$ & $z=c_{3}$ \\
\hline Parameterization & $x_{0}(s)=0$ & $y_{0}(s)=0$ & $z_{0}(s)=Z(s)$ \\
\hline Let $t=0$ and solve for constants & $c_{1}=0$ & $c_{2}=s^{2}$ & $c_{3}=Z(s)$ \\
\hline Replace the constants & $x=t$ & $y=2 A_{0} t+s^{2}$ & $z=Z(s)$ \\
\hline
\end{tabular}

\section{Table 4.3: Method of Characteristics Solution}

From the last row of column three, $s$ can be obtained,

$$
s=\left(y^{2}-2 A_{0} t\right)^{\frac{1}{2}}=\left(y^{2}-2 A_{0} x\right)^{\frac{1}{2}}
$$

Substituting the value of $s$ into column four of Table 4.3 one gets the solution,

$$
z=Z\left(\left[y^{2}-2 A_{0} x\right]^{\frac{1}{2}}\right)
$$

To check the solution from the original equation,

$$
y \frac{\partial z}{\partial x}+A_{0} \frac{\partial z}{\partial y}=0
$$

first compute the derivatives of $z$.

$$
\begin{gathered}
\frac{\partial z}{\partial x}=Z^{\prime}\left(\left[y^{2}-2 A_{0} x\right]^{\frac{1}{2}}\right) \frac{1}{2}\left[y^{2}-2 A_{0} x\right]^{-\frac{1}{2}}\left(-2 A_{0}\right) \\
\frac{\partial z}{\partial y}=Z^{\prime}\left(\left[y^{2}-2 A_{0} x\right]^{\frac{1}{2}}\right) \frac{1}{2}\left[y^{2}-2 A_{0} x\right]^{-\frac{1}{2}}(2 y)
\end{gathered}
$$


Let $K$ be defined by,

$$
K=Z^{\prime}\left(\left[y^{2}-2 A_{0} x\right]^{\frac{1}{2}}\right) \frac{1}{2}\left[y^{2}-2 A_{0} x\right]^{-\frac{2}{2}}
$$

And now equation 4.20 becomes,

$$
y\left(-K 2 A_{0}\right)+A_{0}(K 2 y)=0
$$

Therefor, equation 4.19 is the solution to equation 4.20 subject to the boundary condition described by equation 4.13 . The exact solution to the Vlasov operator,

$$
v \frac{\partial F}{\partial x}+A_{0} \frac{\partial F}{\partial v}=0
$$

with the boundary condition,

$$
F(0, v)=F_{m 0} \exp \left(\frac{-[v-\bar{V}]^{2}}{v_{t h}^{2}}\right)
$$

is therefore,

$$
F(x, v)=F_{m 0} \exp \left(\frac{-\left[\left\{\left(v^{2}-2 A_{0} x\right)^{\frac{1}{2}}\right\}-\bar{V}\right]^{2}}{v_{t h}^{2}}\right)
$$

This exact solution will be compared with numerical solutions in the following section.

\subsubsection{Results}

The first test problem is to transport a Maxwellian ion distribution across space through a constant electric field. This is exactly the problem described by equation 4.25 and equation 4.26, which has the solution given by equation 4.27 . The input values for this problem are given in Table 4.4. From this information $F_{m 0}=n\left(\frac{1}{v_{i n}^{2} \pi}\right)^{\frac{1}{2}}$ can be computed, so the 


\begin{tabular}{|c|c|c|c|c|c|}
\hline \hline$x \max$ & $6 \mathrm{~m}$ & $v \max$ & $2.5 \times 10^{5} \mathrm{~m} / \mathrm{s}$ & $A_{0}$ & $5.0 \times 10^{8} \mathrm{~m} / \mathrm{s}^{2}$ \\
\hline$m$ & 2 AMU's & $T$ & $10 \mathrm{eV}$ & $n$ & $1.0 \times 10^{20} \mathrm{~m}^{-3}$ \\
\hline$V$ & $1.0 \times 10^{5} \mathrm{~m} / \mathrm{s}$ & $f l_{c}$ & 2 & $v_{\text {th }}$ & $31046 \mathrm{~m} / \mathrm{s}$ \\
\hline
\end{tabular}

Table 4.4: Single Maxwellian Problem Input Values

exact solution is known. Figure 4.11 shows the Temperature as a function of distance for the

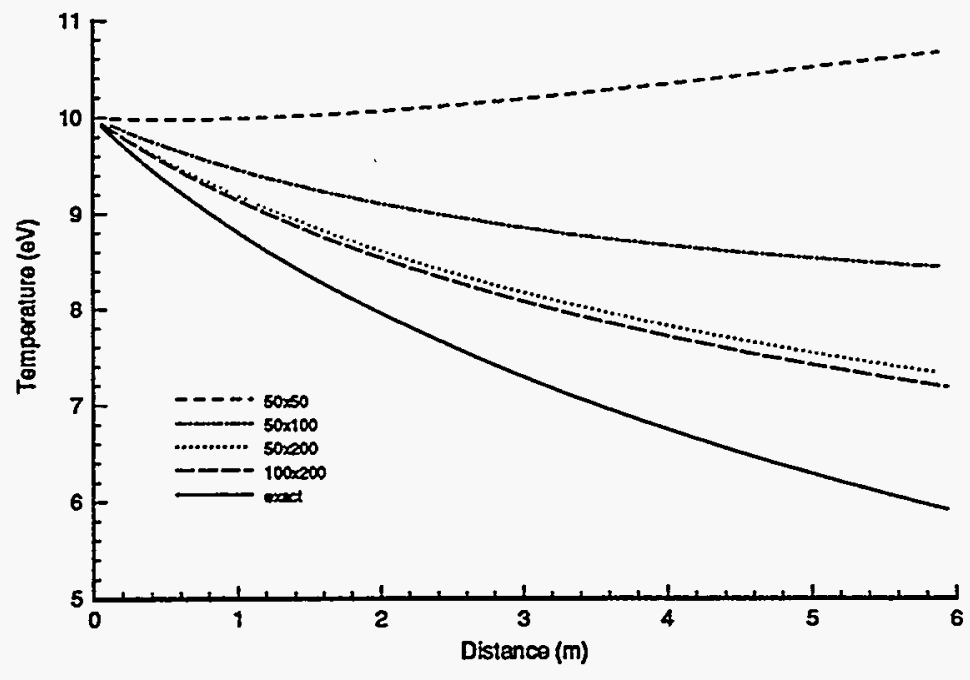

Figure 4.11: Upwind Differencing Temperature Single Maxwellian

single Maxwellian problem for four different computational grids $n x \times n y$. It is clear that the $50 \times 50$ solution is unacceptable, since the temperature appears to increase instead of decreasing. The finer grids yield more accurate solutions, but the large remaining difference between the numerical and exact solutions on the $100 \times 200$ grid indicate that a very fine grid would be required for an accurate solution. Figure 4.12 displays the source of this error by giving the distribution function near $x=6$. This plot shows the shape of the distribution function when it started (west exact), and the shape that it should be when it completes the transit of the computational region (east exact). The upwind differencing solutions have a 


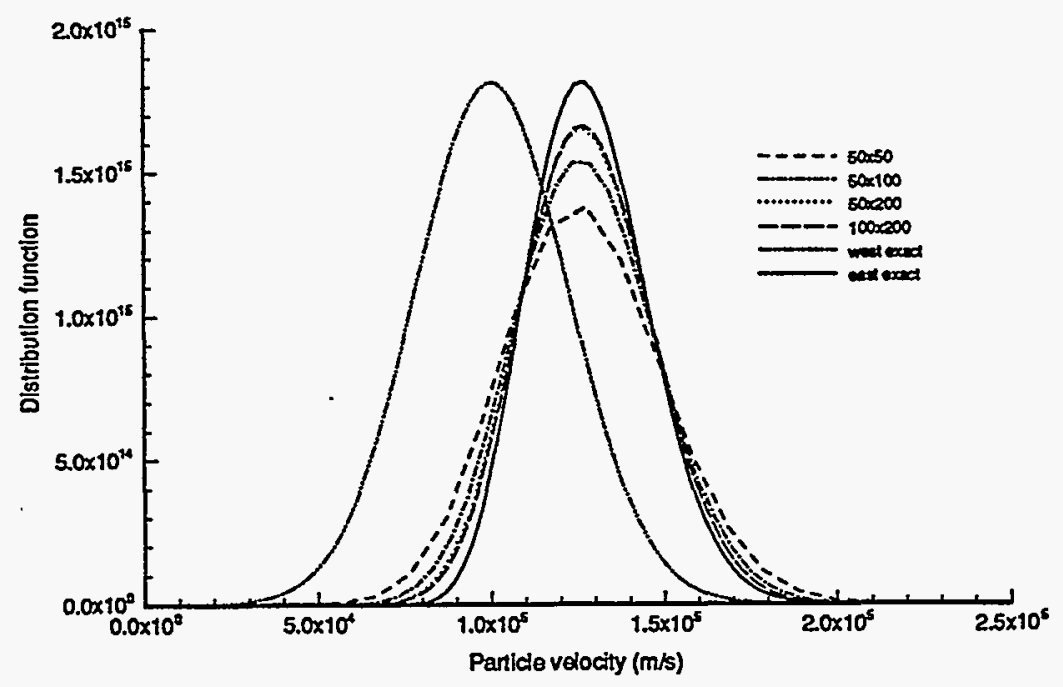

Figure 4.12: Upwind Differencing Distribution Function Single Maxwellian

wider base and a shorter height than the exact solution. Recalling the energy moment,

$$
n k T=m \int_{-\infty}^{\infty}(v-\bar{V})^{2} F d v
$$

one can see that small changes in $F$ away from $\bar{v}$ can have large effects on temperature. Therefore, the seemingly minor numerical diffusion seen in figure 4.12 leads to the large errors shown in figure 4.11 .

By introducing the flux limited QUICK scheme, the results from figure 4.11 are improved to the results of figure 4.13. Here, the coarsest grid results, $50 \times 50$, for the flux limited QUICK scheme are much better than the finest grid, $100 \times 200$, using upwind differencing. The reason for this higher accuracy can be seen in figure 4.14. Here for every grid except the coarsest, the computed solution matches the exact solution for the values of $F$ away from $\bar{V}$ (i.e., everywhere except the peak of the distribution). A comparison in terms of relative 


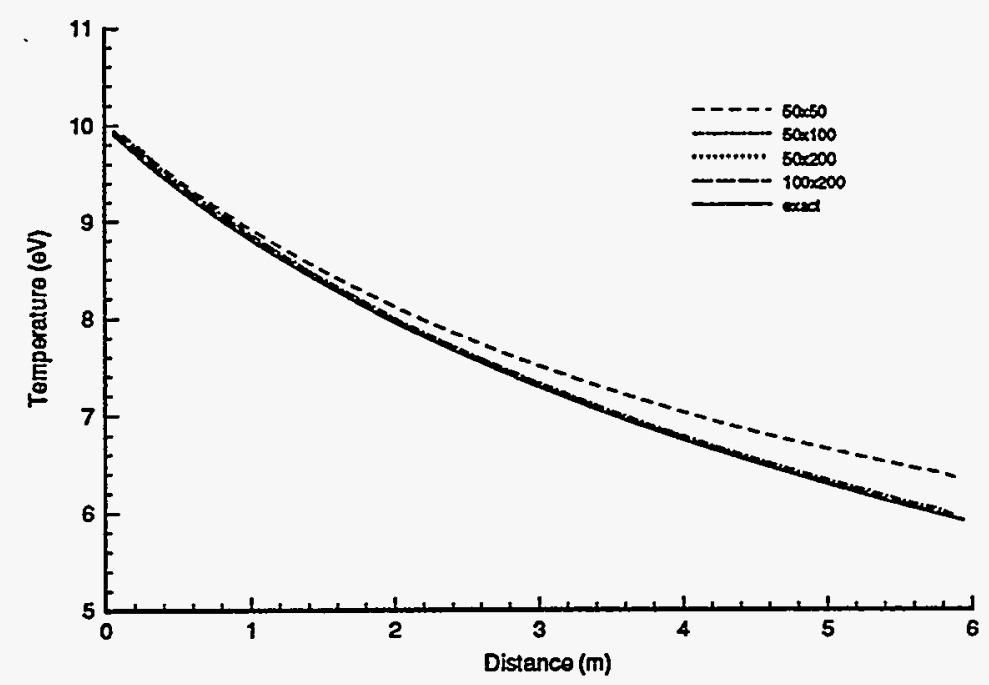

Figure 4.13: Flux Limited QUICK Differencing Temperature Single Maxwellian

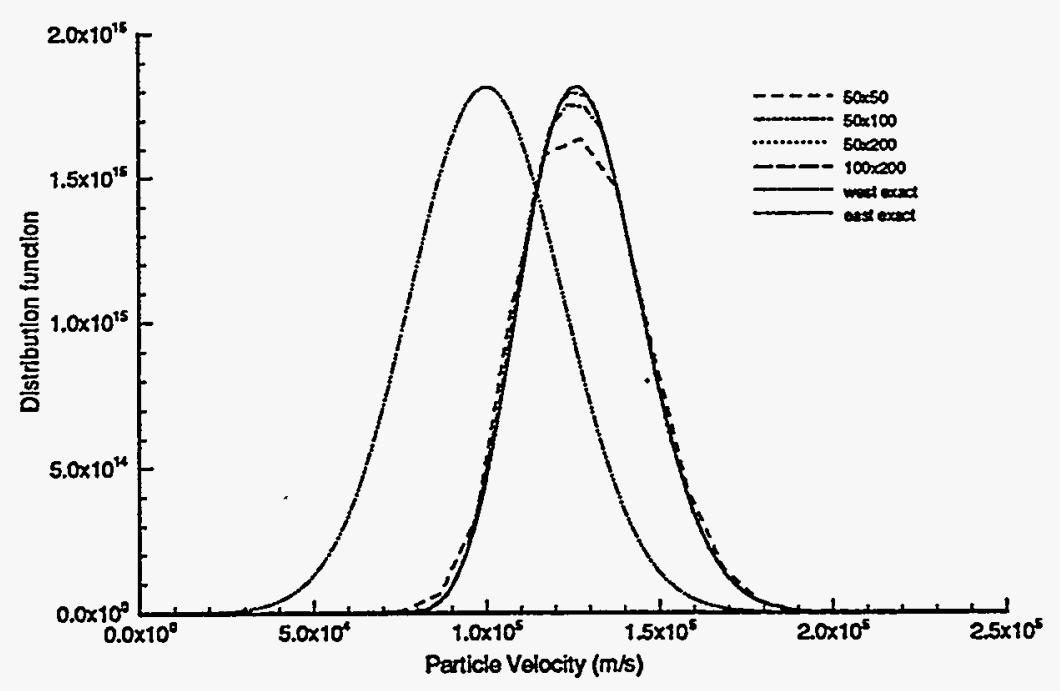

Figure 4.14: Flux Limited QUICK Differencing Distribution Function Single Maxwellian 


\begin{tabular}{|c|c|c|}
\hline \hline nodalization & Upwind & Flux limited QUICK \\
\hline \hline $50 \times 50$ & .794943 & $6.96380 \mathrm{E}-02$ \\
\hline $50 \times 100$ & .421209 & $4.98616 \mathrm{E}-03$ \\
\hline $50 \times 200$ & .2340406 & $7.46582 \mathrm{E}-04$ \\
\hline $100 \times 200$ & .2136886 & $2.09455 \mathrm{E}-06$ \\
\hline \hline
\end{tabular}

Table 4.5: Relative Error for the Single Maxwellian

error of the two methods near the end of the computational domain is presented in Table 4.5. This table clearly indicates not only the higher accuracy, but also the better convergence in terms of number of grid points.

To verify the usefulness of the flux limited QUICK method, other test problems where chosen to show that the differencing works for non-Maxwellian distribution functions. Figure 4.15

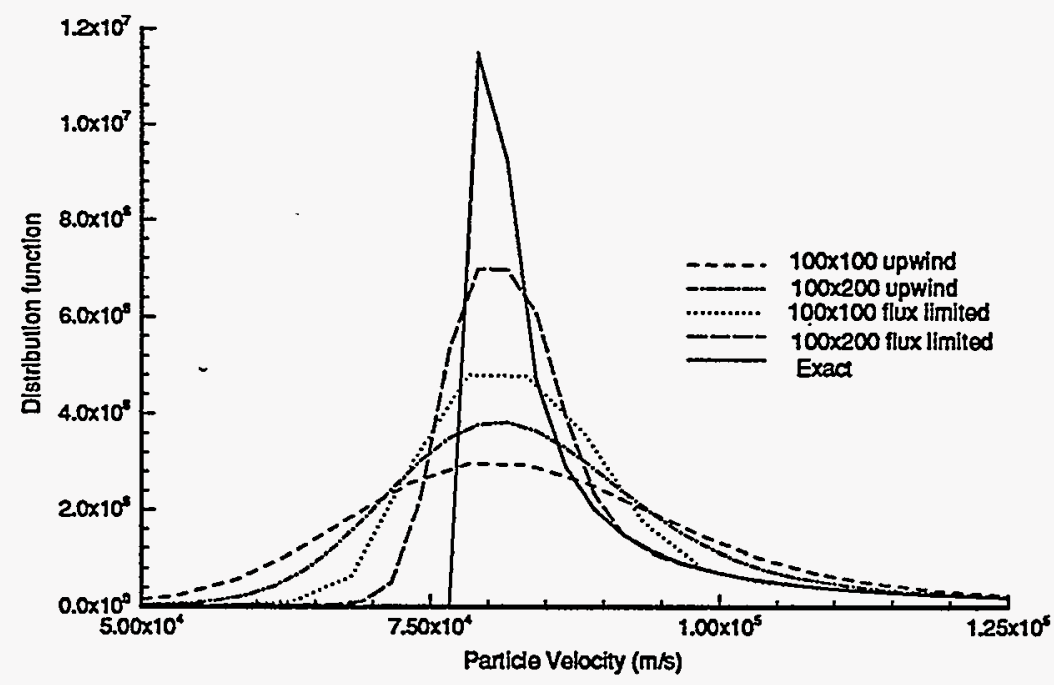

Figure 4.15: Distribution Function for the "Square Wave"

shows results for both flux limited QUICK and upwind differencing on an irregular distribution with a rectangular profile, "square wave". Here only results for the fine meshes are shown. This plot again shows that the flux limited QUICK method has less velocity space 
spread than upwind differencing as shown by the wings of the distribution function.

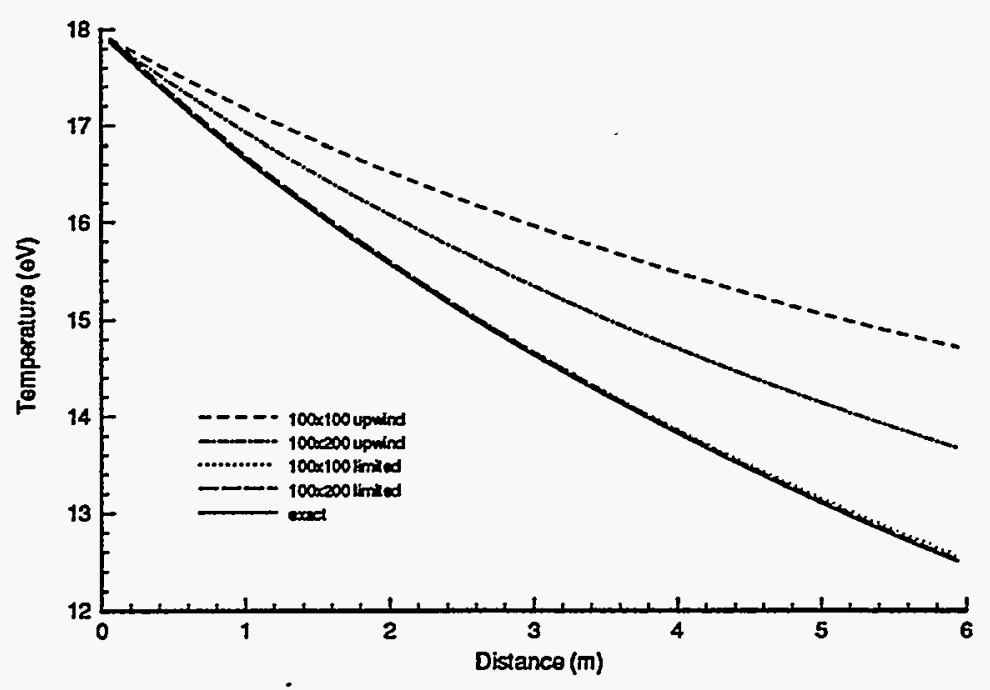

Figure 4.16: Temperature for the Double Maxwellian

The next test problem examined transports two Maxwellian functions across the grid at the same time. The input for this problem is described in Table 4.6. This test problem

\begin{tabular}{|c|c|c|c|c|c|}
\hline \hline$x \max$ & $6 \mathrm{~m}$ & $v \max$ & $2.5 \times 10^{5} \mathrm{~m} / \mathrm{s}$ & $A_{0}$ & $5.0 \times 10^{8} \mathrm{~m} / \mathrm{s}^{2}$ \\
\hline$m$ & $2 \mathrm{AMU} \mathrm{s}$ & $T_{1}$ & $5 \mathrm{eV}$ & $T_{2}$ & $5 \mathrm{eV}$ \\
\hline$n_{1}$ & $1.0 \times 10^{15} \mathrm{~m}^{-3}$ & $n_{2}$ & $1.0 \times 10^{15} \mathrm{~m}^{-3}$ & $V_{1}$ & $1.0 \times 10^{5} \mathrm{~m} / \mathrm{s}$ \\
\hline$V_{2}$ & $1.5 \times 10^{5} \mathrm{~m} / \mathrm{s}$ & $f l_{c}$ & 2 & $\beta$ & 0.0 \\
\hline \hline
\end{tabular}

Table 4.6: Double Maxwellian Problem Input Values

is a hard problem for the differencing scheme because there are many fine structures that must be maintained. Figure 4.16 shows the temperature as a function of time for the double Maxwellian problem for the fine meshes with both upwind and flux limited QUICK differencing. It should be noted that the two flux limited QUICK solutions almost replicate the exact solution. 


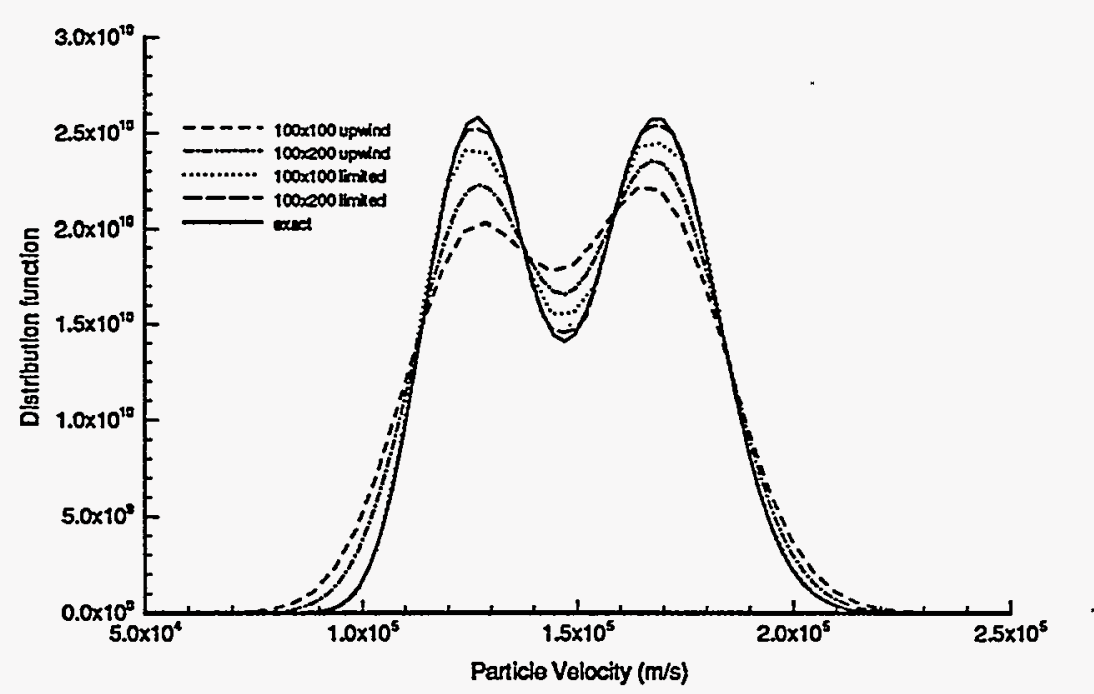

Figure 4.17: Distribution Function for the Double Maxwellian

Figure 4.17 demonstrates the difficulty that upwind differencing has with this test problem. First, it is clear here again that the upwind differencing has spread out the velocity profile in the regions away from the peaks. Second, it is clear that the upwind differencing has radically lowered the peaks and raised the valleys. The third and possibly more disturbing point, is that the symmetry of the problem is lost by the upwind differencing. Clearly the higher velocity Maxwellian has a larger peak than the lower velocity Maxwellian. The relative error of the test problem is shown in Table 4.7. Again the accuracy is much higher and the convergence is much better with flux limited QUICK.

\begin{tabular}{|c|c|c|}
\hline \hline nodalization & Upwind & Flux limited QUICK \\
\hline \hline $100 \times 100$ & .1766881 & $3.24309 \mathrm{E}-03$ \\
\hline $100 \times 200$ & $9.31838 \mathrm{E}-02$ & $6.03242 \mathrm{E}-05$ \\
\hline \hline
\end{tabular}

Table 4.7: Relative Error for the Double Maxwellian 


\subsection{D1V Ion Revisited}

The higher order techniques are now employed to solve the 1D1V ion-only problem from Chapter 2. Now in a more realistic problem, it is more evident that higher order differencing is required. It should be noted the the interpolated donor cell results that were used in Chapter 2 have not been reproduced here.

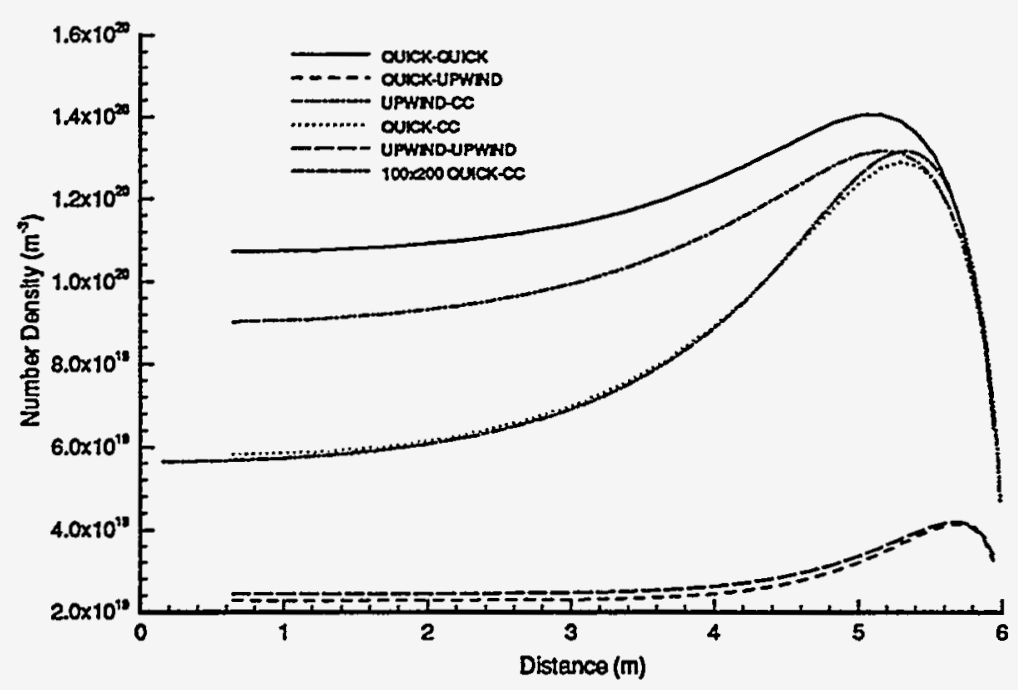

Figure 4.18: Various Discretization Schemes on a $25 \times 50$ Grid

Figure 4.18 shows the results of the $1 \mathrm{D} 1 \mathrm{~V}$ ion-only test problem for five different discretization schemes of the Vlasov and Fokker-Planck operators. These schemes will be represented by an ordered pair where the first element represents the differencing of the Vlasov operator and the second element represents the differencing of the Fokker-Planck operator, i.e. (Vlasov,Fokker-Planck). These results are for a very coarse grid, $25 \times 50$, and a higher order fine mesh, solution, $100 \times 200$, is included for reference. A few observations are obvious in this plot. First, upwind differencing of the Fokker-Planck operator results in unacceptable 
errors as evidenced by the variation of the (QUICK, upwind) and (upwind,upwind) lines from the accurate (QUICK,Chang-Cooper) solution. These two lines are about a factor of three too low in number density. Secondly, even on the coarse $25 \times 50$ grid the (QUICK,ChangCooper) results are very close to the finer grid calculation. Finally, one can see that the (QUICK,QUICK) and (upwind,Chang-Cooper) discretizations deviate from the finer mesh solution farther away from the divertor wall (i.e., near $x=0$ ).

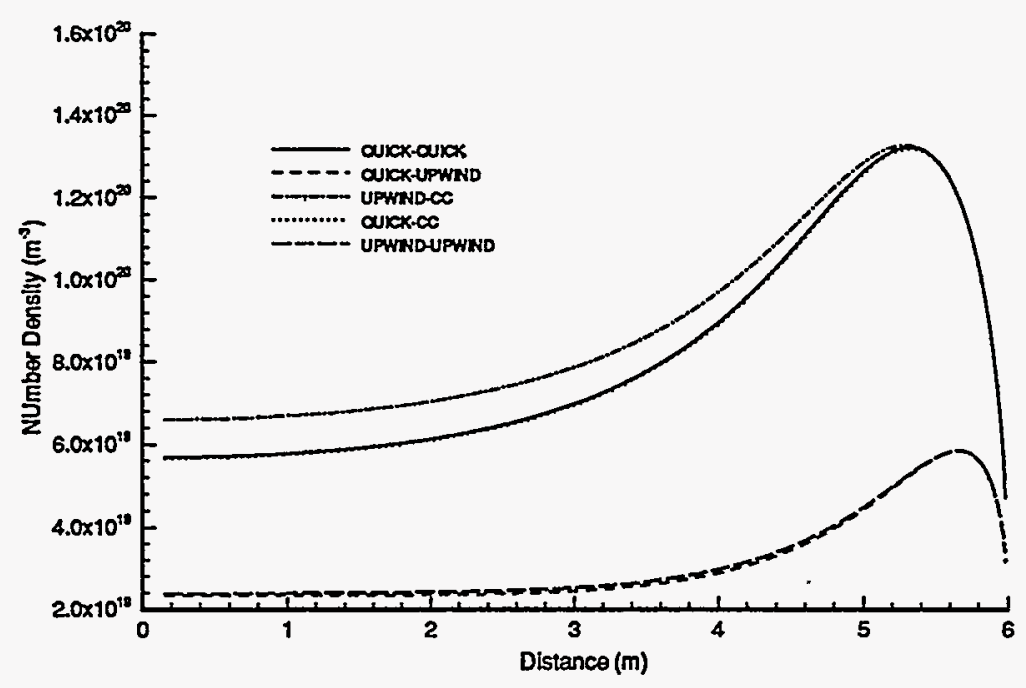

Figure 4.19: Various Discretizätion Schemes on a 100x200 Grid

Figure 4.19 shows the same results as figure 4.18 on a finer grid. The solutions with the upwind Fokker-Planck operator, (QUICK,upwind) and (upwind,upwind), still have not even begun to approach the "correct" solution. The (upwind,Chang-Cooper) results still deviate from the "correct" solution near the symmetry plane (i.e., near $x=0$ ). The (QUICK,QUICK) results however, now lay on top of the (QUICK,Chang-Cooper) solution. It appears from these results that the QUICK scheme may be acceptable for the Fokker-Planck operator as long as the grid is fine enough. 


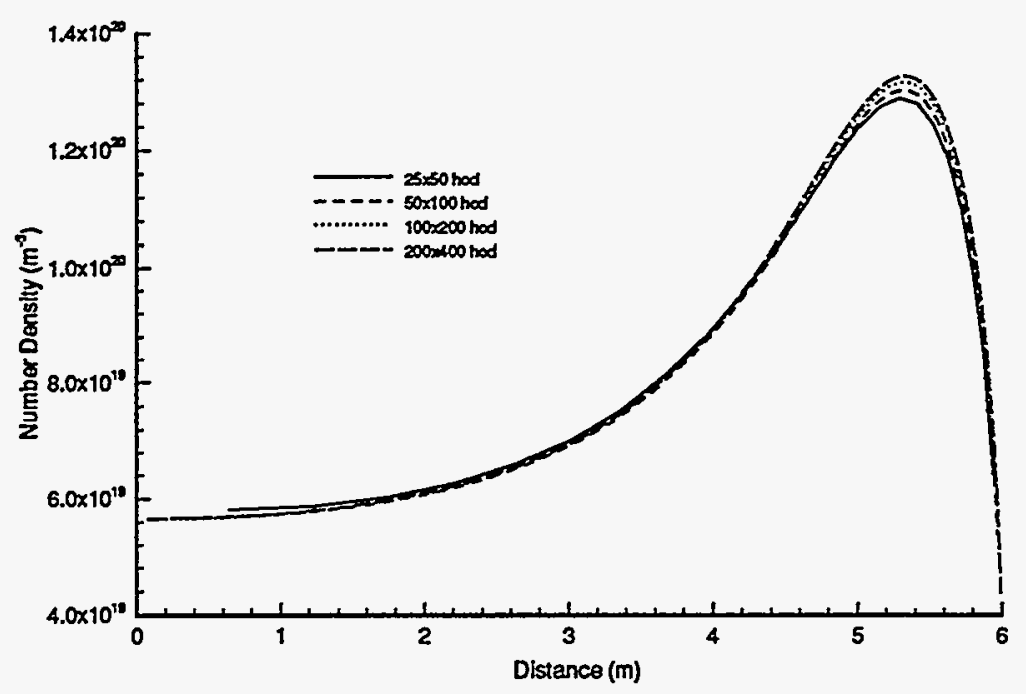

Figure 4.20: Mesh Convergence for QUICK-Chang-Cooper

Figure 4.20 shows the mesh convergence of the (QUICK,Chang-Cooper) differencing method. Here it is evident that even the $25 \times 50$ solution is reasonably close to the $200 \times 400$ solution. Figure 4.21 shows a blow up of the density peak region for three different grids and two different discretization techniques. This plot shows that the solution is both grid independent and method independent. For these runs, the rapid grid convergence of the (QUICK,QUICK) differencing scheme is made evident. Since the $200 \times 400$ solutions of both schemes are the same, this is probably an accurate solution.

\subsection{Summary}

It has been shown that numerical diffusion in the differencing scheme chosen can lead to large errors in integral quantities such as temperature. It has also been shown that by using flux corrected, higher order differencing, mesh independent solutions can be obtained to problems that have very fine structure. 


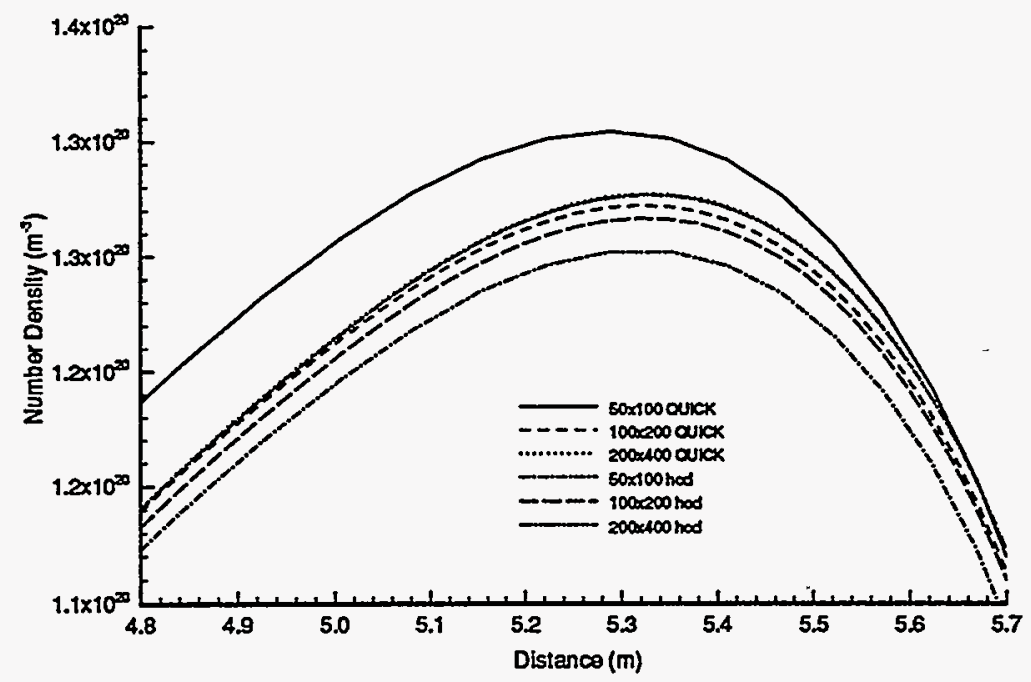

Figure 4.21: QUICK vs. Change-Cooper Collision Differencing 


\section{Chapter 5}

\section{One Velocity One Spatial Ion and}

\section{Electron}

\subsection{Introduction}

In this chapter all of the model improvements from the previous chapters are brought together. The source terms from Chapter 2, the collision operators from Chapter 3, and the higher order differencing from Chapter 4 are combined to solve two new test problems. These test problems represent both high and low neutral particle recycling in a tokamak divertor.

The rest of this chapter has the following organization. Section 5.2 describes the full set of equations being solved. Section 5.3 presents the new electric field equation. Section 5.4 describes the plasma impinging on a surface test problem and presents results from the simulation. Section 5.5 describes a high neutral particle recycling simulation and presents computational results and Section 5.6 summarizes the chapter. 


\subsection{Mathematical Model}

For the 1D1V ion-electron simulations the mathematical model is a superset of the one used in Chapter 2 and that of Chapter 3. The new major addition is the self consistent electric field. The 1D1V VFP equation for ions is,

$$
\frac{\partial F_{i}}{\partial t}+v_{i} \frac{\partial F_{i}}{\partial x}+\frac{q_{i} E}{m_{i}} \frac{\partial F_{i}}{\partial v_{i}}=\left(\frac{\partial F_{i}}{\partial t}\right)_{i i}+\left(\frac{\partial F_{i}}{\partial t}\right)_{i e}+\left(\frac{\partial F_{i}}{\partial t}\right)_{i n z i}+\left(\frac{\partial F_{i}}{\partial t}\right)_{s r c i}
$$

The VFP equation for electrons is now,

$$
\frac{\partial F_{e}}{\partial t}+v_{e} \frac{\partial F_{e}}{\partial x}+\frac{q_{e} E}{m_{e}} \frac{\partial F_{e}}{\partial v_{e}}=\left(\frac{\partial F_{e}}{\partial t}\right)_{e e}+\left(\frac{\partial F_{e}}{\partial t}\right)_{e i}+\left(\frac{\partial F_{e}}{\partial t}\right)_{i n z e}+\left(\frac{\partial F_{e}}{\partial t}\right)_{s r c e}
$$

In this chapter, the neutral ionization terms will be the Maxwellian type from equation 2.40 , not the beam type from equation 2.23. Also for these computations, the source term will not include the velocity dependence that was in equation 2.24 from Emmert, but now it will simply be a Maxwellian source. The collision operators are the same as in Chapter 3 , equation 3.9 through equation 3.12 .

\subsection{Quasi-Neutral Electric Field}

This section develops a new electric field equation, which is different from the one used in Chapter 2 (equation 2.28). From Maxwell's equations one gets,

$$
\epsilon_{0} \nabla \cdot E=e\left(n_{i}-n_{e}\right)
$$


where $\epsilon_{0}$ is the permittivity of free space. If only one spatial dimension is considered, then the equation becomes,

$$
\frac{\partial E}{\partial x}=\frac{e}{\epsilon_{0}}\left(n_{i}-n_{e}\right)
$$

Discretizing this equation with a forward difference and using superscripts to indicate spatial locations yields the following discrete equation,

$$
E^{i+1}=E^{i}+\frac{e \Delta x}{\epsilon_{0}}\left[n_{i}^{i+1}-n_{e}^{i+1}\right]
$$

The problem with using this equation is demonstrated clearly if numerical values in SI units are used. The value of $\frac{e}{\epsilon_{0}}$ is $1.809 \times 10^{-8}(\mathrm{Vm})$. Reasonable values for the number densities, $n i$ and $n e$, are about $1.0 \times 10^{20}\left(m^{-3}\right)$. Assume that, $E^{i+1}-E^{i} \leq 100(\mathrm{~V} / \mathrm{m})$, which is conservative. Also assume that $n_{i}$ and $n_{e}$ can be computed to 5 significant figures. Solving for $\Delta x$ from equation 5.5 results in,

$$
\Delta x=\frac{\left[E^{i+1}-E^{i}\right]}{\frac{e}{\epsilon_{0}}\left[n_{i}^{i+1}-n_{e}^{i+1}\right]}=\frac{100}{\left(1.809 \times 10^{-8}\right)\left(1.0 \times 10^{20}\right)\left(1.0 \times 10^{-5}\right)}=5.5 \times 10^{-6}(\mathrm{~m}) .
$$

This illustrates that for a given a level of numerical error in calculating $n_{i}$ and $n_{e}$, very small $\Delta x$ 's must be used to resolve the electric field. This restriction on $\Delta x$ does not allow for reasonable calculations. Therefore, a different formulation of the electric field is required.

The first attempt at a new statement of the electric field was to modify equation 5.5. Recalling that $\epsilon_{0}=\frac{1}{\mu_{0} c^{2}}$, where $c$ is the speed of light and $\mu_{0}$ is the permeability of free space, one can rewrite equation 5.5 as

$$
E^{i+1}=E^{i}+e \Delta x \mu_{0}(\xi c)^{2}\left[n_{i}^{i+1}-n_{e}^{i+1}\right]
$$


The variable $\xi$ has been included to artifically lower the coefficient on $\left[n_{\dot{i}}^{i+1}-n_{e}^{i+1}\right]$. This method provides reasonable results provided the correct choice of $\xi$ is made. However the choice of $\xi$, makes this electric field statement difficult to use.

An alternative electric field equation comes from the ion and electron momentum equations. Since the electric field appears in both of these momentum equations they can be combined and solved for the electric field. To derive this equation one must start from the ion and electron momentum equations with the inter-particle frictional forces being represented by the last term on the right hand side in both equations.

$$
\begin{aligned}
& m_{i} n_{i} \bar{V}_{i} \frac{\partial \bar{V}_{i}}{\partial x}=q_{i} n_{i} E-\frac{\partial P_{i}}{\partial x}-m_{i} n_{i}\left(\bar{V}_{i}-\bar{V}_{e}\right) \nu_{i e} \\
& m_{e} n_{e} \bar{V}_{e} \frac{\partial \bar{V}_{e}}{\partial x}=\dot{q}_{e} n_{e} E-\frac{\partial P_{e}}{\partial x}-m_{e} n_{e}\left(\bar{V}_{e}-\bar{V}_{i}\right) \nu_{e i}
\end{aligned}
$$

Recalling that $\nu_{e i}=\left[\frac{m_{i} n_{i}}{m_{e} n_{e}}\right] \nu_{i e}$, one can see that the last term in equation 5.8 and equation 5.9 are equal and opposite as they should be since they represent the same force. Using the fact that $q_{i}=-q_{e}=e$ and subtracting equation 5.9 and equation 5.8 the following equation is obtained,

$$
E=\frac{1}{e\left(n_{i}+n_{e}\right)}\left[m_{i} n_{i} \bar{V}_{i} \frac{\partial \bar{V}_{i}}{\partial x}-m_{e} n_{e} \bar{V}_{e} \frac{\partial \bar{V}_{e}}{\partial x}+\frac{\partial P_{i}}{\partial x}-\frac{\partial P_{e}}{\partial x}-2 m_{i} n_{i}\left(\bar{V}_{i}-\bar{V}_{e}\right) \nu_{i e}\right]
$$

If one assumes that $n_{i} \cong n_{e}$ and $\bar{V}_{i} \cong \bar{V}_{e}$ everywhere except in the frictional term, one ends up with the following quasi-neutral electric field equation,

$$
E=\frac{1}{2 e n_{i}}\left[2\left(m_{i}-m_{e}\right) n_{i} \bar{V}_{i} \frac{\partial \bar{V}_{i}}{\partial x}+\frac{\partial P_{i}}{\partial x}-\frac{\partial P_{e}}{\partial x}-2 m_{i} n_{i}\left(\bar{V}_{i}-\bar{V}_{e}\right) \nu_{i e}\right]
$$


Equation 5.11 will be used to evaluate the electric field in this chapter rather than equation 2.28 from Chapter 2. This equation is less restrictive for $\Delta x$ than equation 5.3 since the spatial resolution is now based on the fluid velocity and pressure and not the speed of light.

\subsection{Test 1: Plasma Impinging on a Surface}

The first test problem in this section is chosen to check the fully-coupled solution and the electric field calculation. In this simulation, the volumetric source is the largest source of particles, although there is a small number of neutrals coming off the wall. The input parameters are shown in Table 5.1.

\begin{tabular}{|c|c|c|c|c|c|}
\hline \hline$n x$ & 25 & $n y$ & 50 & $L$ & $6.0 \mathrm{~m}$ \\
\hline$v_{\operatorname{maxi}}$ & $2.5 \times 10^{5} \mathrm{~m} / \mathrm{s}$ & $v_{\operatorname{maxe}}$ & $2.5 \times 10^{6} \mathrm{~m} / \mathrm{s}$ & $T_{i n j}$ & $75.0 \mathrm{eV}$ \\
\hline$m_{e}$ & $0.02 \mathrm{AMU} ' \mathrm{~s}$ & $m_{i}$ & $2 \mathrm{AMU} \mathrm{s}$ & $R_{i n j}$ & $1.0 \times 10^{24} \mathrm{~m}^{-3} \mathrm{~s}^{-1}$ \\
\hline$v_{n}$ & $1.0 \times 10^{5} \mathrm{~m} / \mathrm{s}$ & $f_{n}(L)$ & $4.5 \times 10^{18} \mathrm{~m}^{-3}$ & $L_{i n j}$ & $4.83 \mathrm{~m}$ \\
\hline$\lambda_{n}$ & $1.0 \mathrm{~m}$ & $T_{n}$ & $5 \mathrm{eV}$ & $\Delta x_{\min }$ & $0.06 \mathrm{~m}$ \\
\hline \hline
\end{tabular}

Table 5.1: Input Values for Ion-Electron Plasma Impinging on a Wall

In the first plot, figure 5.1, one can see the characteristic drop off in number density as the plasma approaches the wall. One can also see that the plasma is quasi-neutral, $n_{i} \cong n_{e}$, except for a small region caused by the source terms near $x=0$. The electric field is shown in figure 5.2. It should be noted that the electric field is set equal to zero at the left symmetry boundary. Because of this boundary condition, the electric field does not reflect the small non-quasineutral condition in the number densities at the same location. One can see that the electric field is very small away from the wall and grows rapidly as the plasma approaches the wall. The shape of the electric field is typical of a pre-sheath. The electric field provides two features to the simulation. First, it maintains quasi-neutrality up to the 


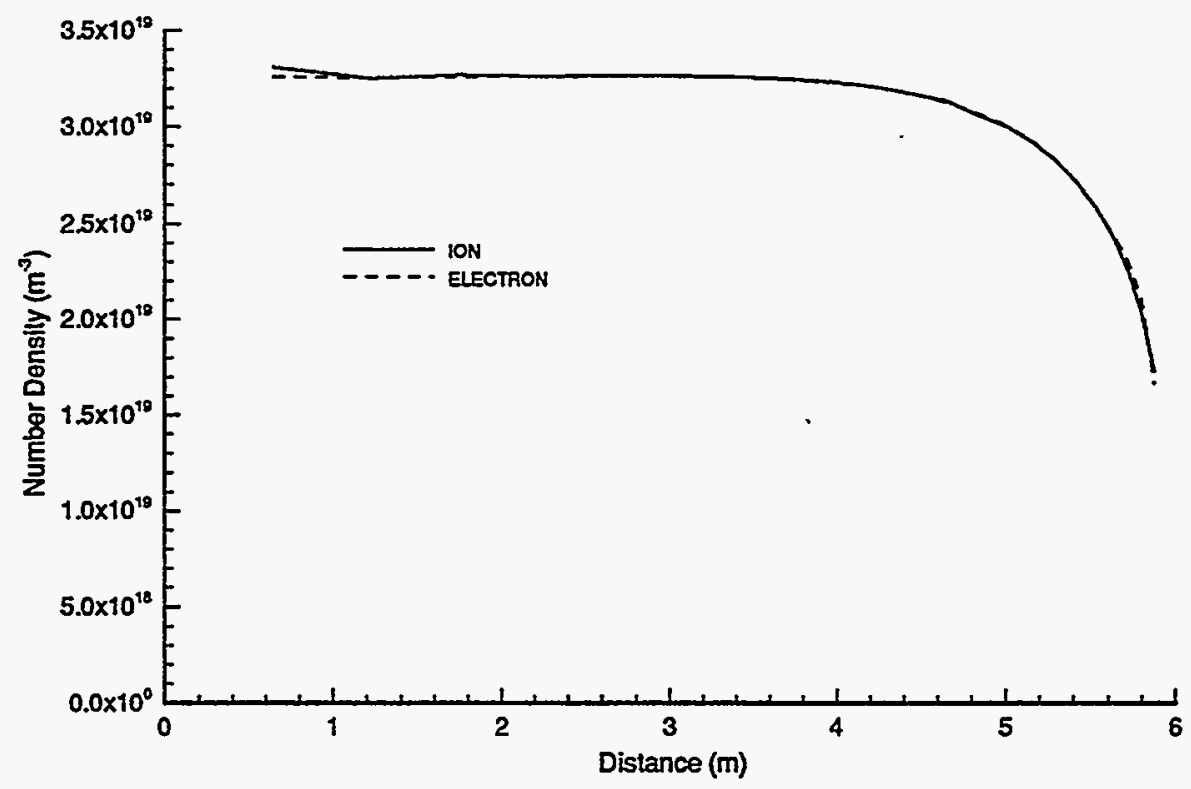

Figure 5.1: Number Densities on the 25x50 Grid

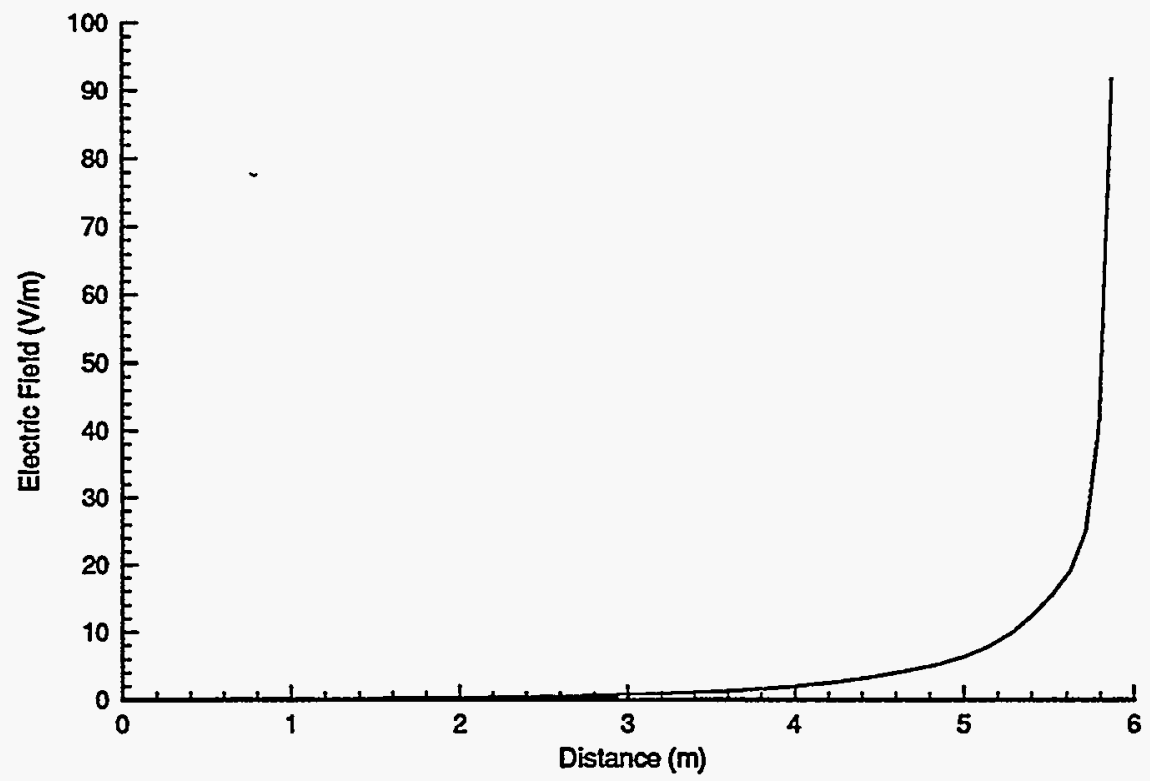

Figure 5.2: Electric Field on the 25x50 Grid 
sheath, as demonstrated in Fig 5.1, and second, it accelerates the ions and decelerates the electrons so the particle fluxes are equal. The particle fluxes are shown in figure 5.3 and they overlay exactly and are clearly equal. Note that there is a slight bump in the particles fluxes near five meters which is caused by the end of the core source injection region which is 4.83 $\mathrm{m}$ long. Figure 5.4 shows the fluid velocity of the ions and electrons, which also overlay.

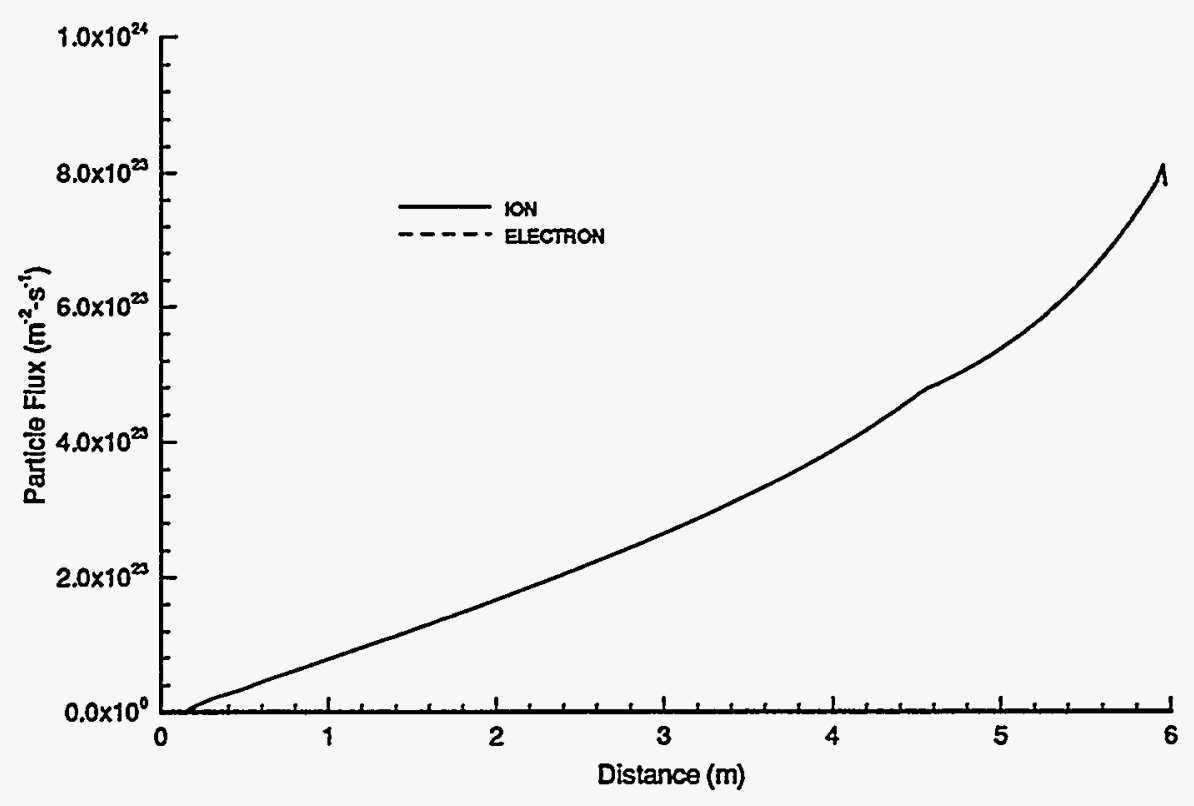

Figure 5.3: Particle Flux on the $100 \times 200$ Grid

According to the Bohm sheath criteria [73], the ion velocity should be greater than the sound speed in the plasma. The speed of sound in a plasma is given by Chen [12] as,

$$
v_{s}=\left(\frac{\gamma_{e} k T_{e}+\gamma_{i} k T_{i}}{m_{i}}\right)^{\frac{1}{2}}
$$

where $\gamma_{e}$ is the ratio of specific heats for the electrons and $\gamma_{i}$ is the ratio of specific heats for the ions. For these plots this is simplified to $v_{s}=\left(\frac{2 k T_{i}}{m}\right)^{\frac{1}{2}}$. In figure 5.5 one can see that the 


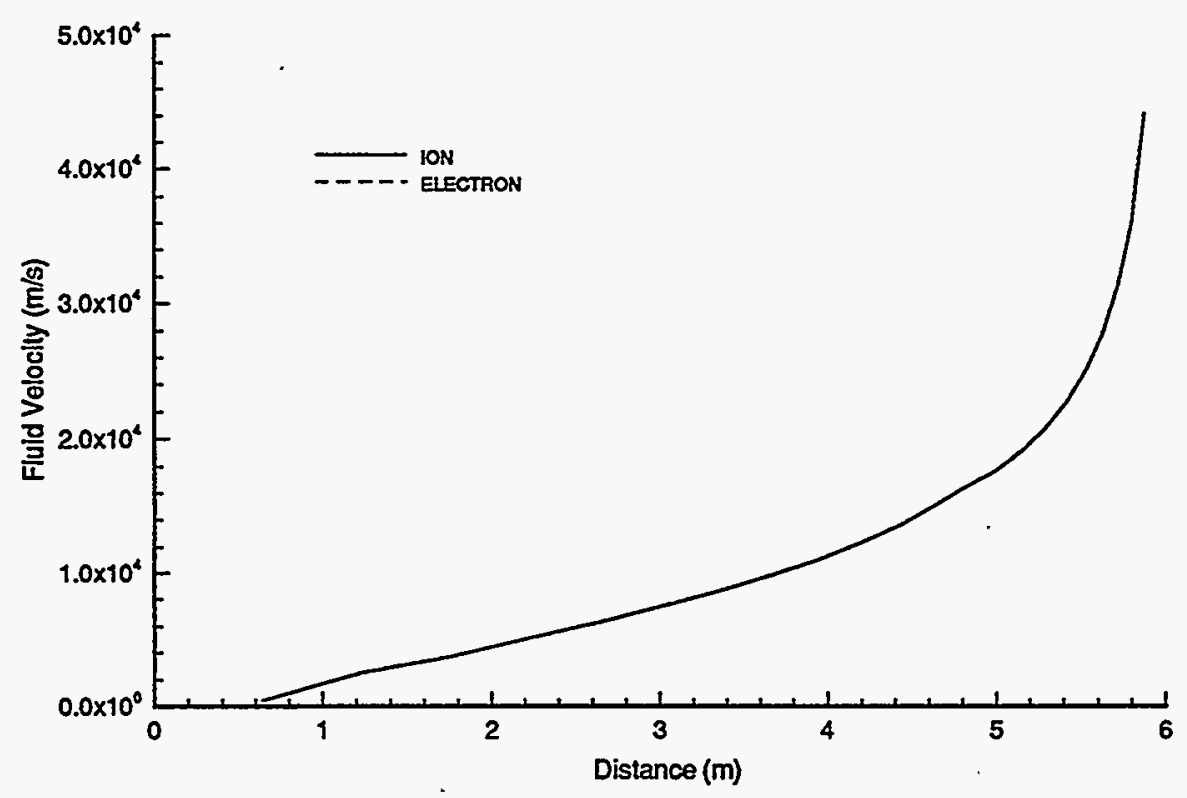

Figure 5.4: Fluid Velocity on the $25 \times 50$ Grid

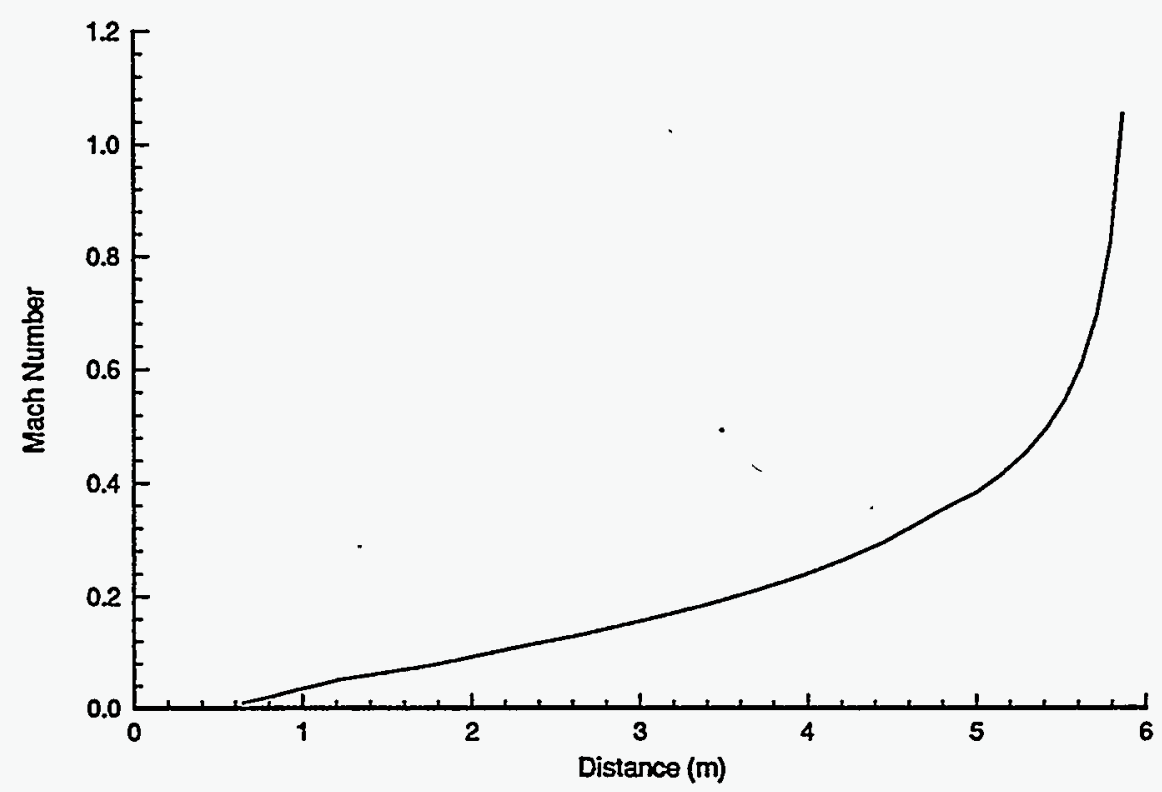

Figure 5.5: Mach Number on the 25x50 Grid 
Mach number of the plasma as it reaches the sheath is slightly larger than one.

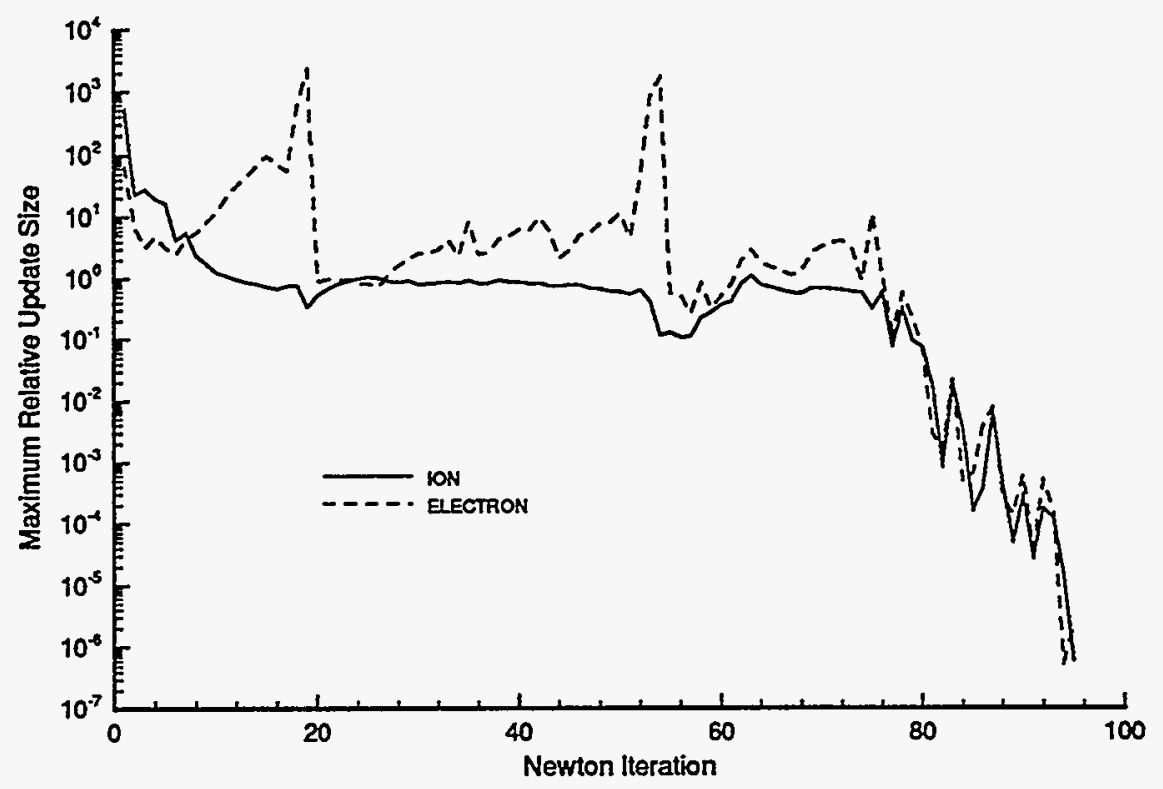

Figure 5.6: Newton Convergence on the $25 \times 50$ Grid

Figure 5.6 shows the convergence of the method. This plot is not as smooth as similar plots shown for the ion-only simulations, but the full ion-electron problem is not yet optimized. Figure 5.7 shows the mesh sequenced Mach number plots. One can see that except for fine resolution very near the wall and boundary condition effects near the left symmetry plane, that the $25 \times 50$ grid gives reasonably accurate solutions. Figure 5.8 shows the convergence on the three different meshes as a function of CPU time on a HP 735 workstation. Here one can see that a fully converged solution on a 100×200 grid can be obtained without any human intervention in under ten hours on a standard workstation with 128 Meg of RAM. Convergence is defined from the Newton linearization step, equation 2.32, which has been 


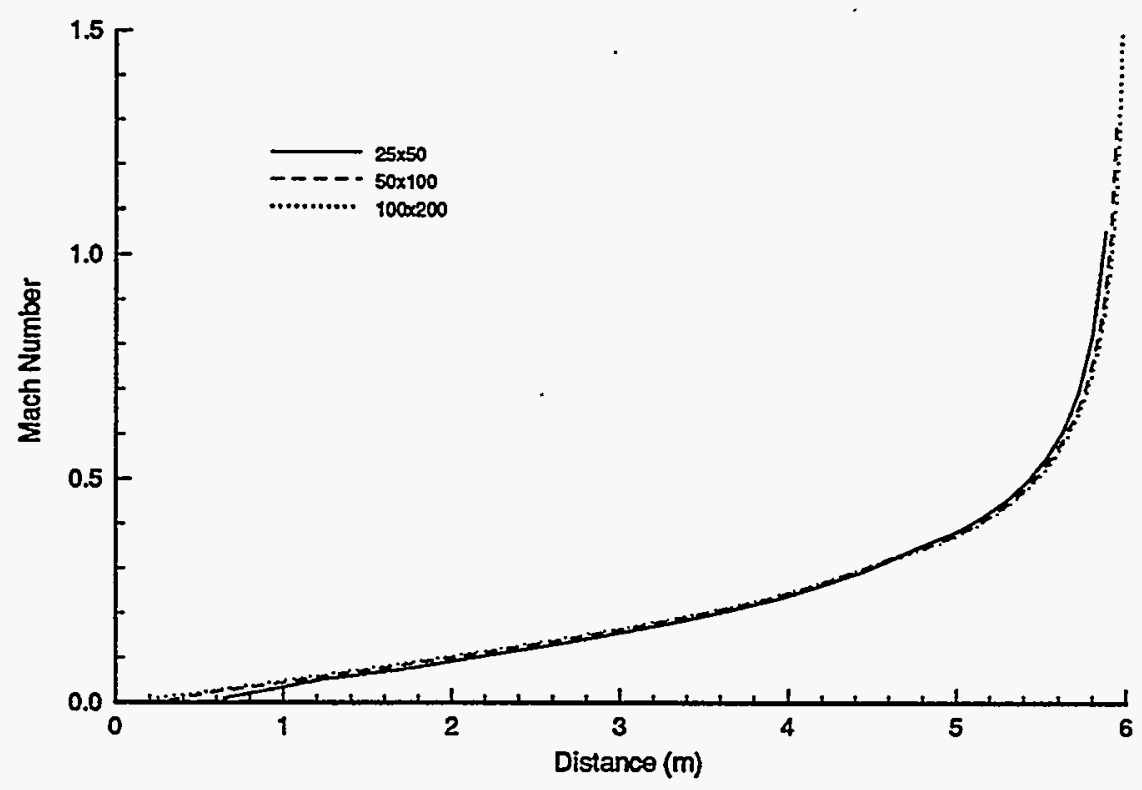

Figure 5.7: Mesh Sequenced Mach Numbers

repeated here for clarity.

$$
\mathbf{J}^{n} \delta \mathbf{y}^{n}=-\mathbf{G}\left(\mathbf{y}^{n}\right)
$$

This iteration is continued until $\left|\delta \mathrm{y}^{n}\right|<1.0 \times 10^{-5}$. Figure 5.8 also shows that for scoping studies, where less accuracy is required, answers can be obtained with a coarse grid $(25 \times 50)$ in under 20 minutes on the same platform.

\subsection{Test 2: High Recycling}

For this test problem more neutral particles are reflected back off the divertor plate, to demonstrate how the solution changes with higher particle recycling. Comparing Table 5.1 with Table 5.2 one can see that difference between these two runs is only in the neutral model. Figure 5.9 clearly shows that the temperature gradients are much steeper for the low 


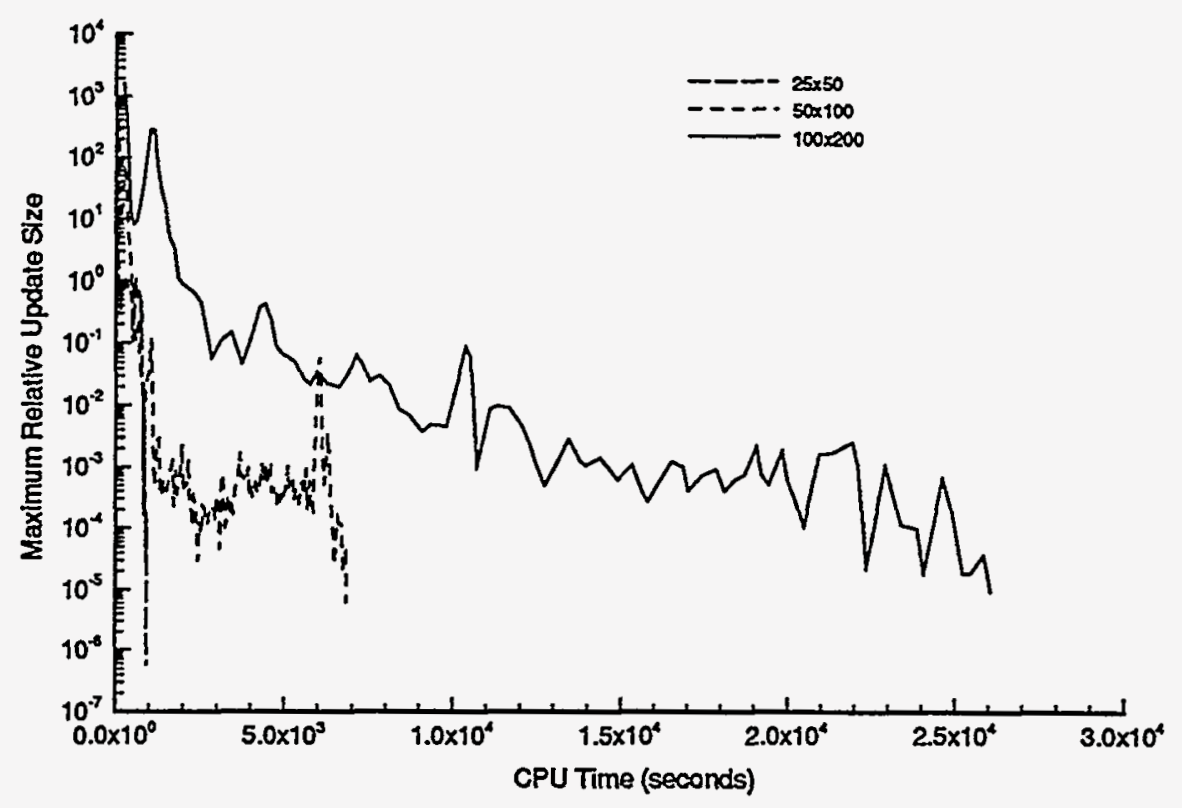

Figure 5.8: Mesh Sequenced Newton Convergence for the Ion Equation

\begin{tabular}{|c|c|c|c|c|c|}
\hline \hline$n x$ & 25 & $n y$ & 50 & $L$ & $6.0 \mathrm{~m}$ \\
\hline$v_{\operatorname{maxi}}$ & $2.4 \times 10^{5} \mathrm{~m} / \mathrm{s}$ & $v_{\operatorname{maxe}}$ & $2.4 \times 10^{6} \mathrm{~m} / \mathrm{s}$ & $T_{i n j}$ & $75.0 \mathrm{eV}$ \\
\hline$m_{e}$ & $0.02 \mathrm{AMU} \mathrm{s}$ & $m_{i}$ & $2 \mathrm{AMU} / \mathrm{s}$ & $R_{i n j}$ & $1.0 \times 10^{24} \mathrm{~m}^{-3} \mathrm{~s}^{-1}$ \\
\hline$v_{n}$ & $3.5 \times 10^{4} \mathrm{~m} / \mathrm{s}$ & $f_{n}(L)$ & $1.0 \times 10^{20} \mathrm{~m}^{-3}$ & $L_{i n j}$ & $4.83 \mathrm{~m}$ \\
\hline$\lambda_{n}$ & $0.5 \mathrm{~m}$ & $T_{n}$ & $5 \mathrm{eV}$ & $\Delta x_{\min }$ & $0.06 \mathrm{~m}$ \\
\hline \hline
\end{tabular}

Table 5.2: High Recycling Simulation 


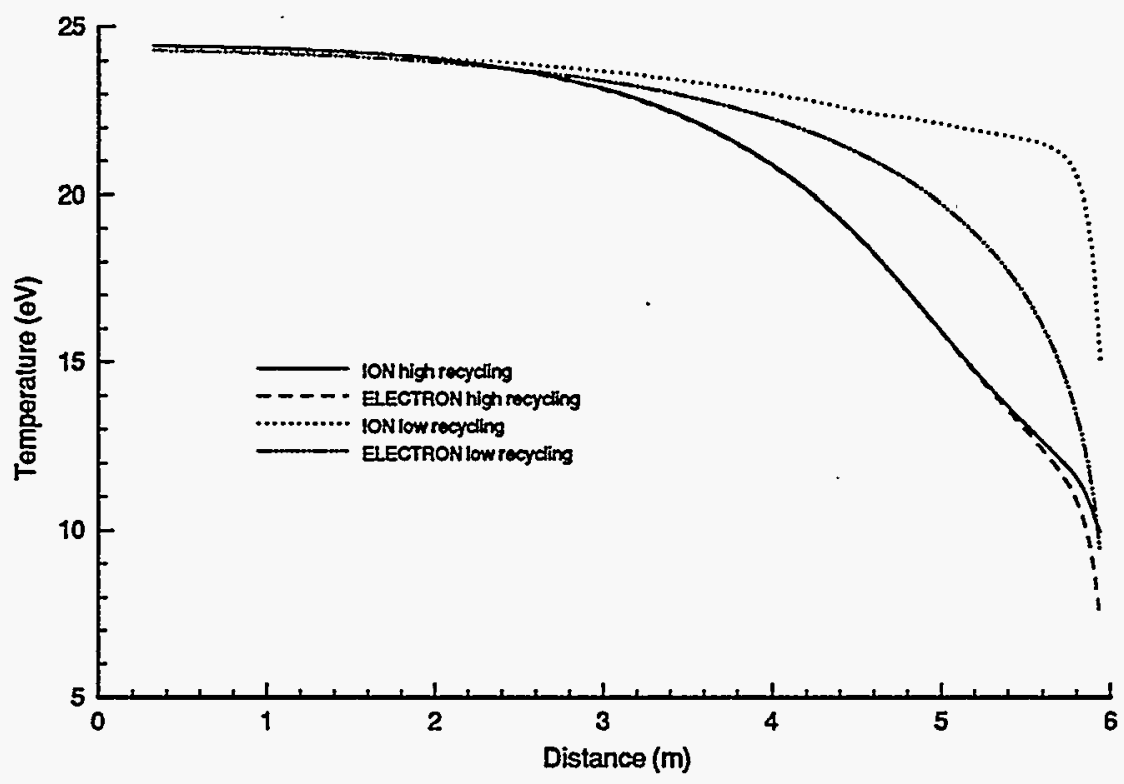

Figure 5.9: Ion and Electron Temperatures for High and Low Recycling Rate for Neutrals recycling problem. Figure 5.10 shows the difference in the distribution functions for the high and low recycling cases. Because of the symmetry boundary condition at $x=0$, the number density is much higher for the high recycling case. From figure 5.1 and figure 5.11 one can see that the upstream densities differ by about a factor of four, $1.4 \times 10^{20}$ vs. $3.3 \times 10^{19}$.

Figure 5.12 shows the collision frequencies for the high recycling simulation. From this plot, one can see the wide variation in collision time scales which result from equation 3.14 through equation 3.17 of Chapter 3 . One can see that the slow time scale, electrons colliding with ions, is about $6.5 \times 10^{4} \mathrm{~s}^{-1}$ near the symmetry plane, and the fast time scale, electrons colliding with electrons, is about $2.7 \times 10^{7} \mathrm{~s}^{-1}$ near the plate. Note that the $\nu_{e e}$ and the $\nu_{e i}$ lines exactly overly each other. The reason for this is obvious from equation 3.17 when $n_{i}=n_{e}$. This wide range in time scales is what makes these problems difficult to solve 


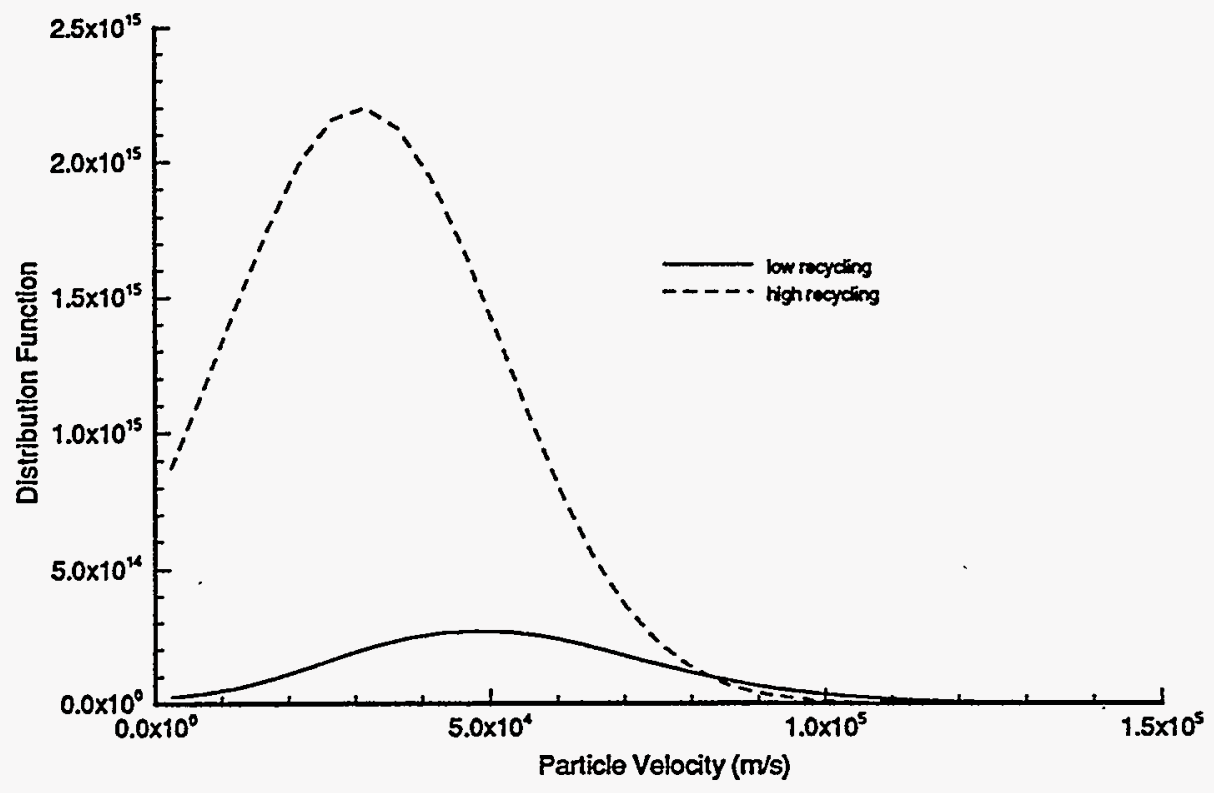

Figure 5.10: Half Distribution Function for High and Low Recycling

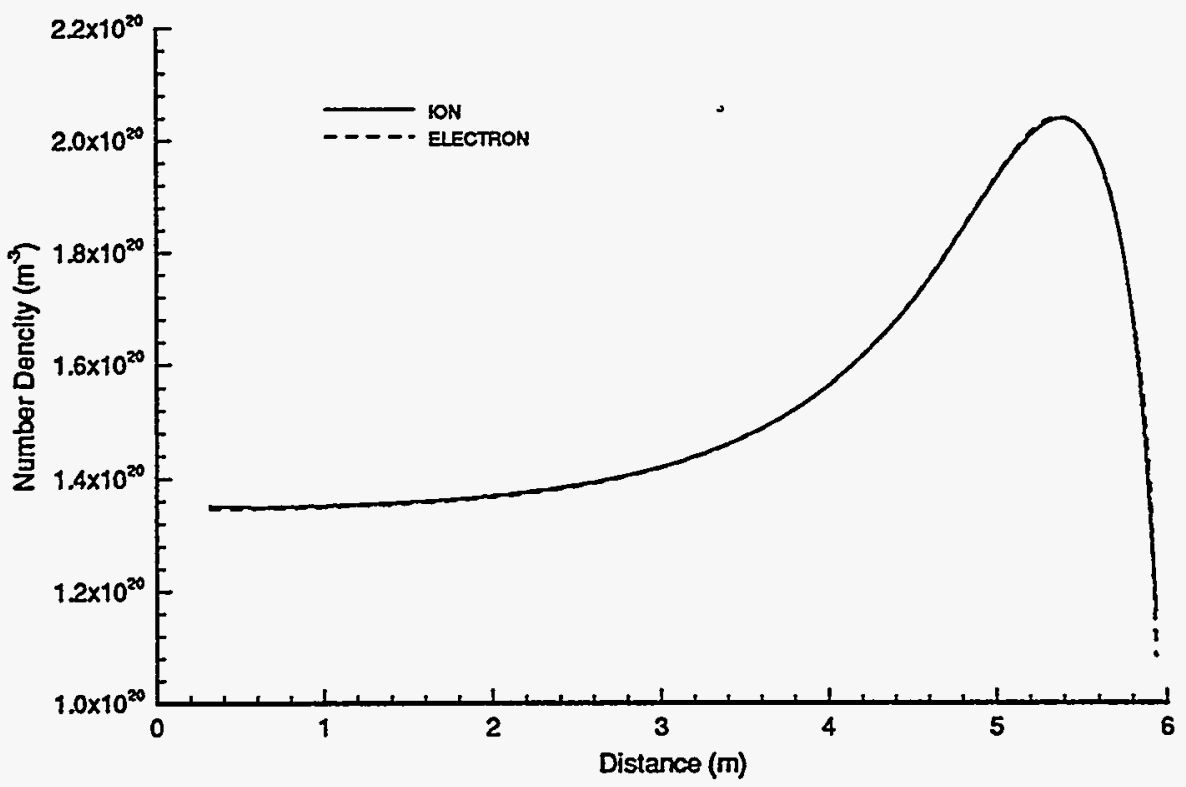

Figure 5.11: High Recycling Number Densities on the 50x100 Grid 
numerically.

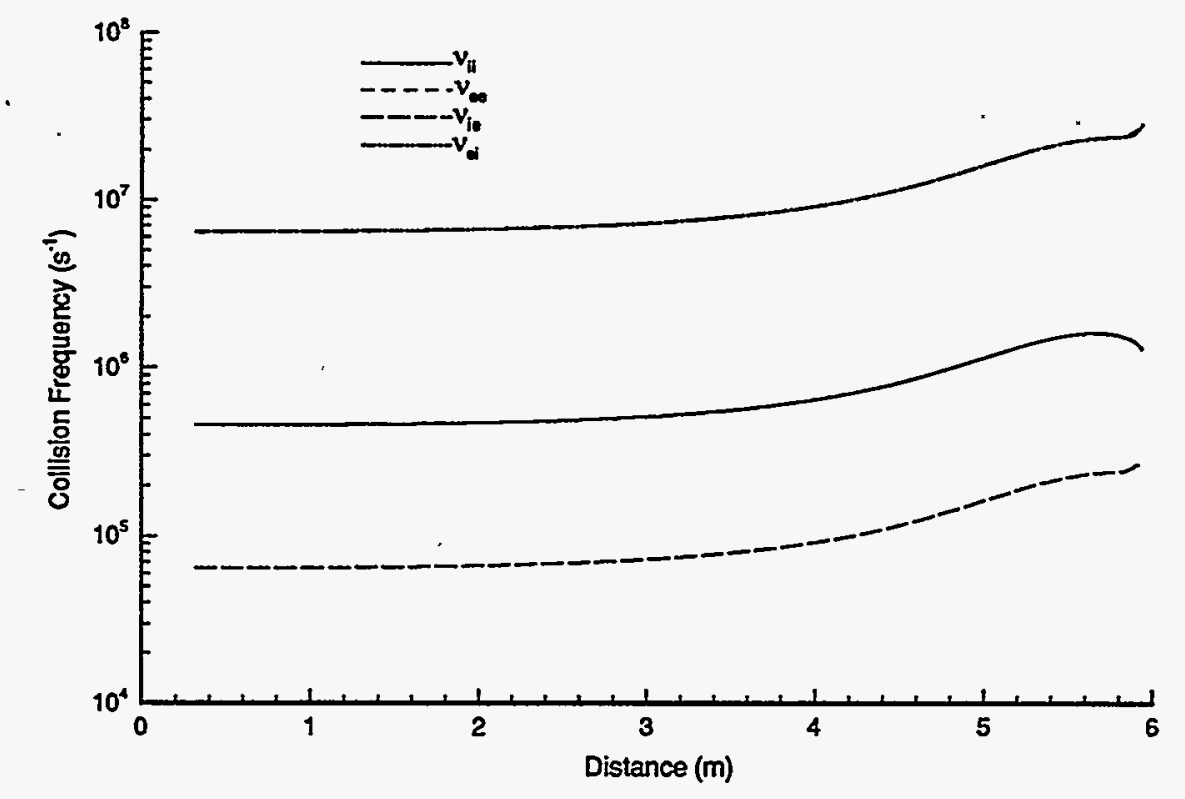

Figure 5.12: High Recycling Collision Frequencies

\subsection{Summary}

In this chapter all of the results from the previous chapters are combined to obtain a fully coupled ion-electron problem with a self consistent electric field. Simulations are presented for both a high and low neutral particle recycling rates on a tokamak divertor. These results demonstrate that the Matrix-Free Newton-Krylov method is an effective technique for solving kinetic particle transport simulations at both high and low recycling rates. 


\section{Chapter 6}

\section{Summary}

\subsection{Summary and Conclusions}

A new numerical technique, Matrix-Free Newton Krylov, has been demonstrated to be an efficient method for solving the Vlasov-Fokker-Planck equations. This numerical method has a very low memory requirement and a very rapid convergence rate. These features make solving a large number of problems on a workstation a viable option.

Direct discretization of the Vlasov-Fokker-Planck equation has been an intimidating task in the past. Many researchers have found that discretization errors can overwhelm the simulation that they are trying to run. It has been shown in this work that flux-limited, QUICK differencing of the Vlasov operator and Chang-Cooper differencing of the Fokker-Planck operator result in accurate solutions as long as a large enough number of cells are used.

The Matrix-Free Newton Krylov method, coupled with advanced differencing, allows the solution of coupled ion-electron problems on workstations. Using the quasi-neutral electric field allows one to obtain quasi-neutral solutions on a large spatial grid, even with the smallest grid spacing many orders of magnitude larger than the Debye length. 


\subsection{Future Work}

There are three areas that should be investigated further; a non-orthogonal mesh capability, improved collision operators, and an improved neutral particle model. Advantages of a more generalized mesh capability are discussed first. As shown in the previous sections, for simplified divertor simulations there are wide variations in temperature from tens of electron volts, for the streaming plasma, to the one electron volt range, at the divertor surface. It has been found that three to five $v_{t h}$ works well as the maximum velocity for the particle velocity dimension discretization. The velocity range must be large enough to resolve the particle velocity dependence of the distribution function, but if the range is too large the value of the distribution function approaches zero. Accurate computing with these small distribution function values is numerically difficult. Since the thermal velocity has a $T^{1 / 2}$ dependency, there is a large change in the optimal grid size as the temperature drops near a solid surface. Because of this, in the simulations of this dissertation the high temperature injection regions are well resolved, but there are difficulties resolving the low temperature regions near a divertor plate. To overcome this difficulty, one could use a non-orthogonal grid. The wide grid spacing near the core could resolve the high plasma temperature region, while the narrow grid spacing near the plate could resolve the low plasma temperatures there. Implementation of this strategy would require modification to the equations of this dissertation to account for the non-orthogonal grid.

In addition, one would like to improve the collision operators. The operators used in this work are an accurate first order treatment, but they could be improved. The collision frequency should include a particle velocity dependence. In addition, a new Boltzmann collision operator should be included to account for the ionization energy lost from the electrons to 
ionize the neutrals.

Finally an improved neutral model should be investigated. In the new model one would want to include a full kinetic description of the neutrals and a better representation of the wall and its temperature. These suggested improvements are left for future researchers. 
Appendix A

Taylor Series for Central and

Upwind Differencing

A.1 Forward, Backward, and Upwind

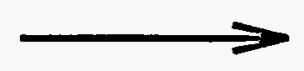

$\mathbf{u}_{\mathbf{i}}$
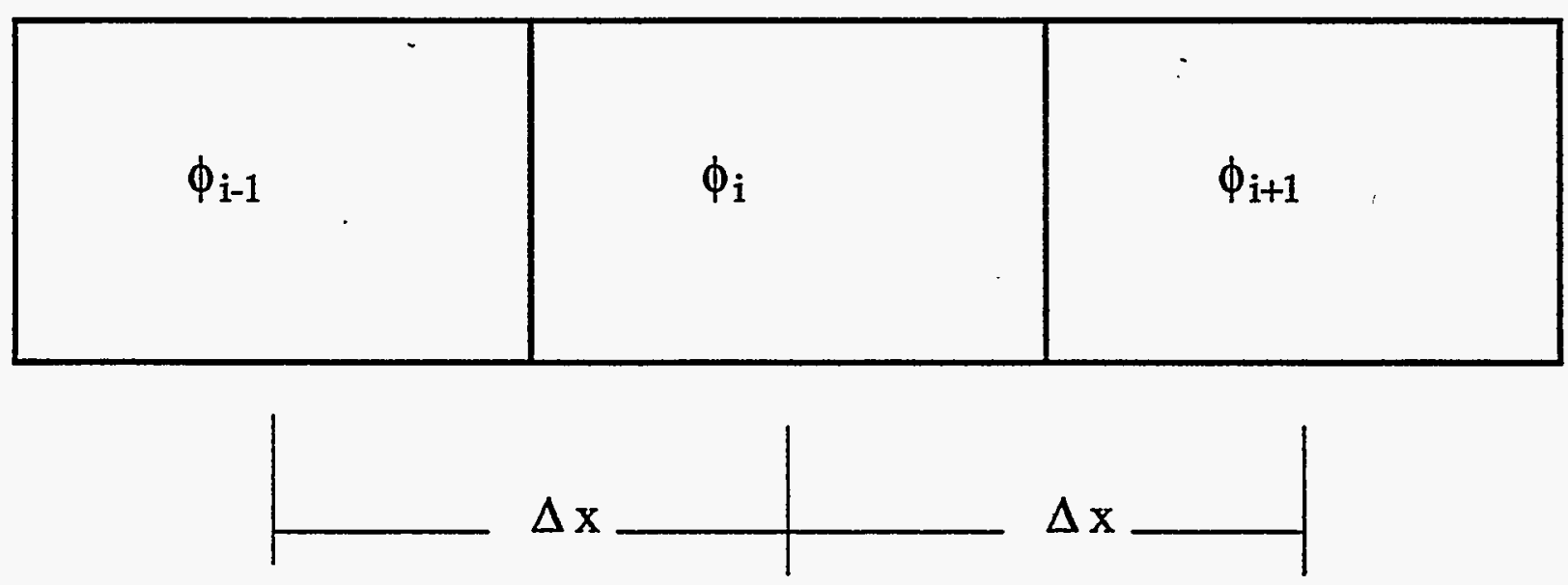

Figure A.1: Upwind Discretization Mesh 
The forward difference Taylor series is given by,

$$
\phi_{i+1}=\phi_{i}+\left.\frac{\partial \phi}{\partial x}\right|_{i} \Delta x+\left.\frac{1}{2} \frac{\partial^{2} \phi}{\partial x^{2}}\right|_{i} \Delta x^{2}+\left.\frac{1}{6} \frac{\partial^{3} \phi}{\partial x^{3}}\right|_{i} \Delta x^{3}+H O T
$$

where the terms are described in figure. A.1 and HOT stands for higher order terms. From this one gets the forward difference approximation,

$$
\left.\frac{\partial \phi}{\partial x}\right|_{i F}=\frac{\phi_{i+1}-\phi_{i}}{\Delta x}-\left.\frac{1}{2} \frac{\partial^{2} \phi}{\partial x^{2}}\right|_{i} \Delta x+H O T
$$

or,

$$
\left.\frac{\partial \phi}{\partial x}\right|_{i F}=\frac{\phi_{i+1}-\phi_{i}}{\Delta x}+O(\Delta x)
$$

where $O(\Delta x)$ means terms that are first order or higher in $\Delta x$, e.g. $\Delta x, \Delta x^{2}, \Delta x^{3}, \ldots$ The backward difference Taylor series is given by,

$$
\phi_{i-1}=\phi_{i}-\left.\frac{\partial \phi}{\partial x}\right|_{i} \Delta x+\left.\frac{1}{2} \frac{\partial^{2} \phi}{\partial x^{2}}\right|_{i} \Delta x^{2}-\left.\frac{1}{6} \frac{\partial^{3} \phi}{\partial x^{3}}\right|_{i} \Delta x^{3}+H O T
$$

which results in the following difference formula,

$$
\left.\frac{\partial \phi}{\partial x}\right|_{i B}=\frac{\phi_{i}-\phi_{i-1}}{\Delta x}+O(\Delta x)
$$

Considering the convective derivative, $u \frac{\partial \phi}{\partial x}$, one can define upwind differencing as,

$$
\left.u \frac{\partial \phi}{\partial x}\right|_{i U}=\left\{\begin{array}{cc}
\left.u \frac{\partial \phi}{\partial x}\right|_{i F} & u>0 \\
\left.u \frac{\partial \phi}{\partial x}\right|_{i B} & u<0
\end{array} .\right.
$$




\section{A.2 Central Differencing}

Central differencing is obtained from subtracting equation. A.4 from equation. A.1. This results in,

$$
\phi_{i+1}-\phi_{i-1}=\left.2 \frac{\partial \phi}{\partial x}\right|_{i} \Delta x+\left.\frac{1}{3} \frac{\partial^{3} \phi}{\partial x^{3}}\right|_{i} \Delta x^{3}+H O T .
$$

Therefore, the central differencing approximation is,

$$
\left.\frac{\partial \phi}{\partial x}\right|_{i C}=\frac{\phi_{i+1}-\phi_{i-1}}{2 \Delta x}+O\left(\Delta x^{2}\right)
$$




\section{Appendix B}

\section{Differentiable Flux Limiter}

\section{Derivation}

\section{B.1 Introduction}

It was found early in this work that the use of a non-differentiable flux limiter led to stalling of the Newton convergence process. A modification to the flux limiter was developed to correct this problem. At points of discontinuous derivative, the flux limiter was replaced by a cubic spline fit of the bounding linear segments. Another alternative approach to correcting the lack of convergence is the use of under-relaxation. It should be noted that this differentiable flux limiter only is applicable for constant mesh spacings (i.e., $\Delta x$ and $\Delta v$ are constant).

This appendix has the following sections. First, the under-relaxation option is be briefly discussed. Next, the dimensional limiter equations are non-dimensionalized. Following is a presentation of the work to smooth the limiter in non-dimensional space. Finally the implementation of the new limiter in dimensional space is discussed. 


\section{B.2 Under-relaxation}

The under-relaxation method for eliminating the convergence stalling is easy to understand and implement. Consider figure B.1 which is reproduced from Section 4.3.2, and suppose that the solution is oscillating around point 3. Without loss of generality, assume that the actual solution is on line $3-4$. If the method is oscillating about point 3 then the real solution will be either on line $2-3$ or line $3-4$. Without damping, the solution can jump back and forth from line 2-3 to line 3-4. If the update is damped however, the new iteration will not move from line 3-4 to line 2-3. If the iterations stay on line $3-4$, the correct solution will be quickly found. This damping of the iterations can be accomplished by limiting the

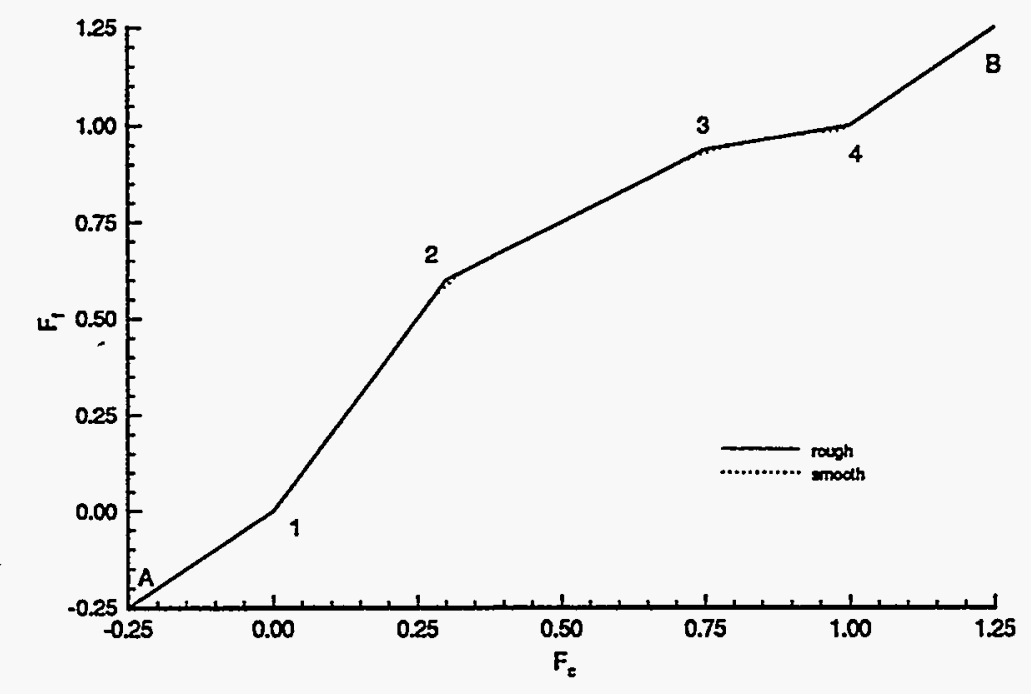

Figure B.1: Differentiable (Smooth) and Non-Differentiable (Rough) Limiters

maximum size of the update, or by simply using only a fraction of the new update. A more efficient method may be to damp the solution only when convergence stalling is detected. In Chapter 4 examples were given of stalled convergence, figure 4.9 and figure 4.10. Figure 4.9 
will be repeated here for clarity (see Section 4.3 .2 for details). In this plot, one can see that

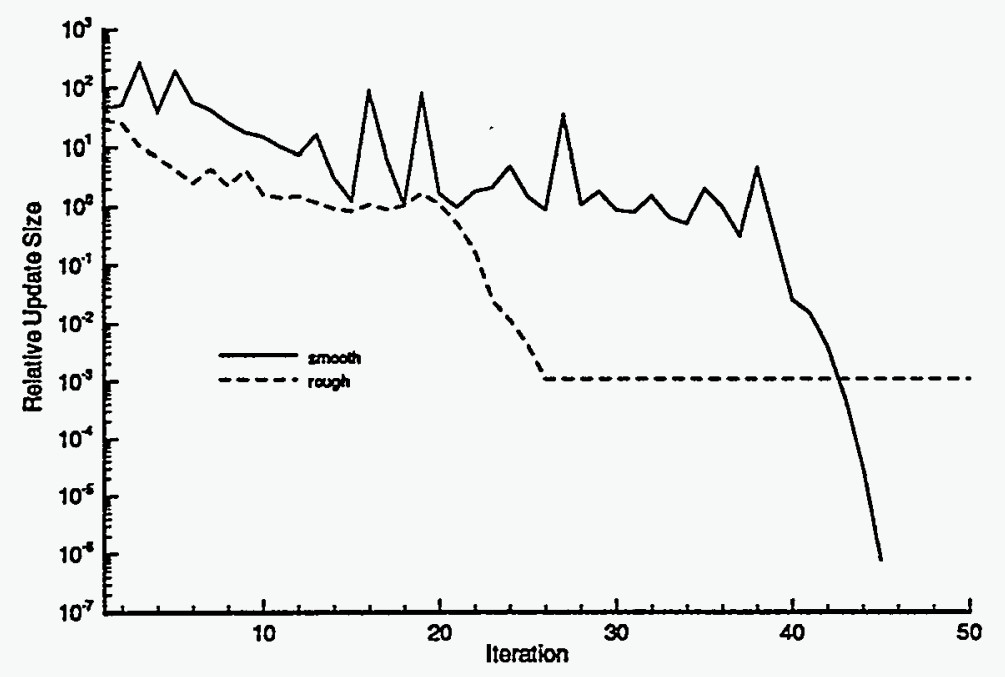

Figure B.2: Differentiable vs. Non-Differentiable Limiter Convergence for a Steady State Run

the relative update size for the "rough" limiter is approximately a constant for all Newton Iterations beyond 30. Therfore, one could detect convergence stall if the update size does not change over three or four Newton iterations.

Another approach is to turn off the limiting when it causes a convergence stall. The following modification of the limiter is only one of many approaches to correct this problem. This smoothing approach is probably not the most computationally efficient method, but it is very easy to understand and is robust in nature.

There is one caveat to the use of smoothing. If the update size is large and the area that has been smoothed is small, the oscillations may still occur, as it will be possible to jump over the smoothed region. 


\section{B.3 Non-Dimensional Non-Differentiable}

The first step in smoothing the flux limiter is to convert the equations to non-dimensional form. The two equations used to convert from dimensional space to non-dimensional space are,

$$
\begin{aligned}
& \tilde{F}_{f}=\frac{F_{f}-F_{U}}{F_{D}-F_{U}} \\
& \tilde{F}_{C}=\frac{F_{C}-F_{U}}{F_{D}-F_{U}} .
\end{aligned}
$$

From figure B.1 one can see that there are four lines that need non-dimensional equations. The first is line $\overline{A B},(\overline{A 1}, \overline{4 B})$,

$$
F_{f}=F_{C}
$$

The second line $\overline{12}$ is,

$$
F_{f}=F_{U}+f l_{c}\left(F_{C}-F_{U}\right)=\left(1-f l_{c}\right) F_{U}+f l_{c} F_{C}
$$

The third line, $\overline{23}$, is the higher order differencing line or the QUICK line. Note that for this derivation and the examples given in this dissertation the uniform grid spacing version of the QUICK operator is used. The derivation would be more complicated for the non-uniform method and would proceed as follows. The functional form of the QUICK method would be expressed as,

$$
F_{f}=A F_{U}+B F_{C}+C F_{D}
$$


This converts to non-dimensional form as,

$$
\tilde{F}_{f}=B \tilde{F}_{C}+C
$$

For this implementation equation B.6 will be different for each cell face because each $A, B$, and $C$ are different, because they all depend on the local cell spacing. The uniform grid version of the dimensional QUICK line is,

$$
F_{f}=-\frac{1}{8} F_{U}+\frac{3}{4} F_{C}+\frac{3}{8} F_{D}
$$

The fourth line, $\overline{34}$ is given by,

$$
F_{f}=\beta F_{C}+(1-\beta) F_{D}
$$

From equation B.5 and equation B.6 one can see the general pattern to convert the dimensional equation into the non-dimensional equation. Therefore, the four dimensional equations,

$$
\begin{aligned}
& F_{f}=F_{C} \\
& F_{f}=\left(1-f l_{c}\right) F_{U}+f l_{c} F_{C} \\
& F_{f}=-\frac{1}{8} F_{U}+\frac{3}{4} F_{C}+\frac{3}{8} F_{D} \\
& F_{f}=\beta F_{C}+(1-\beta) F_{D}
\end{aligned}
$$


become the four non-dimensional equations,

$$
\begin{aligned}
\tilde{F}_{f} & =\tilde{F}_{C} \\
\tilde{F}_{f} & =f l_{c} \tilde{F}_{C} \\
\tilde{F}_{f} & =\frac{3}{4} \tilde{F}_{C}+\frac{3}{8} \\
\tilde{F}_{f} & =\beta \tilde{F}_{C}+(1-\beta) .
\end{aligned}
$$

\section{B.4 Non-Dimensional Differentiable}

The following is a description of the non-dimensional equations used to smooth the limiter. Assumptions will be stated, but not always justified. The four points to be smoothed are labeled in figure B.1. It should be noted that the full detail of the differences between the smooth and the rough limiter are not clear in this figure, but it is included to give a global presentation and "close ups" of the smoothed areas will be presented later. For point 1, one of the points will be $(0,0)$, for point 4 , one of the points will be $(1,1)$. This is done to insure that the limiter line stays in the Convection Boundedness Criterion triangle as discussed in Section 4.3.2.

Figure B.3 shows a blow up of the area around point 1 . At the point $(0,0)$, the slope is 1 since the equation for $F_{C}<0$ is,

$$
F_{f}=F_{C}
$$

The equation for $F_{C}>0$ is,

$$
F_{f}=f l_{c} F_{C}
$$

It is safe to assume $f l_{c}>1$ since $f l_{c}=1$ is simply upwind differencing. In figure B.3 the 


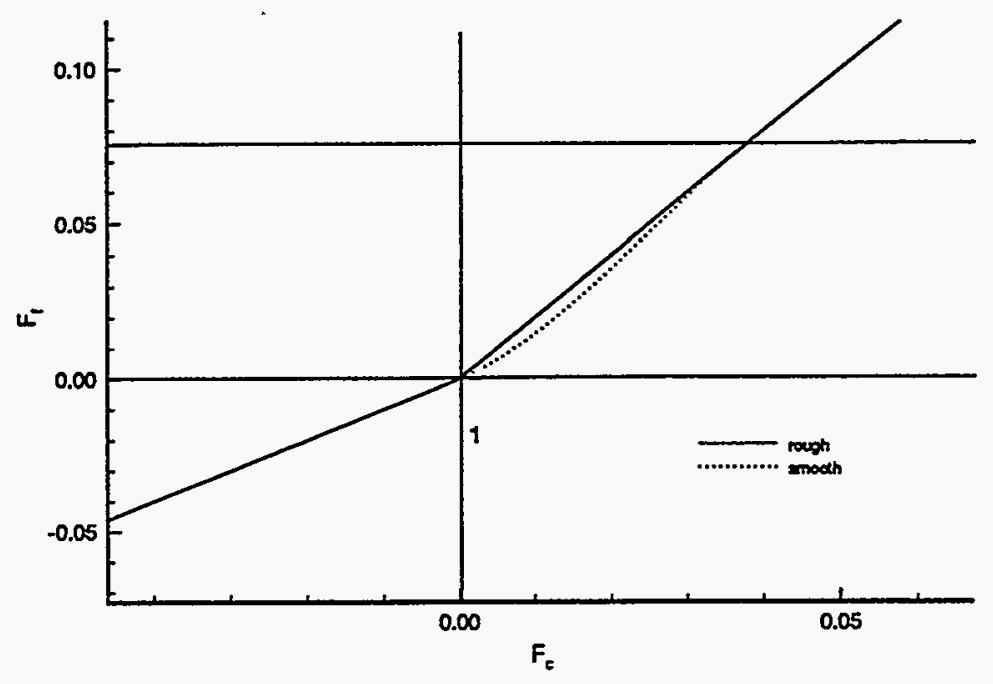

Figure B.3: Differentiable and Non-Differentiable Limiters point 1

amount of limiter smoothing, tol, was set at 0.075 . Therefore, the point to cut the $f l_{c}$ line is determined by where that line intersects a box $(2$ tol $\times 2$ tol $)$ centered at $(0,0)$. Since $f l_{c}>1$, this intersection takes place at $F_{f}=t o l$ and $F_{C}=\frac{t o l}{f l_{c}}$. At the point $\left(\frac{t o l}{f l_{c}}, t o l\right)$ the slope is $f l_{c}$. We now have the two points and the two slopes necessary to define a cubic spline. The cubic function,

$$
a F_{C}^{3}+b F_{C}^{2}+c F_{C}+d=F_{f}
$$

is defined by solving the following matrix equation,

$$
\left[\begin{array}{cccc}
3 X_{H}^{2} & 2 X_{H} & 1 & 0 \\
X_{H}^{3} & X_{H}^{2} & X_{H} & 1 \\
3 X_{L}^{2} & 2 X_{L} & 1 & 0 \\
X_{L}^{3} & X_{L}^{2} & X_{L} & 1
\end{array}\right]\left[\begin{array}{c}
a \\
b \\
c \\
d
\end{array}\right]=\left[\begin{array}{c}
S_{H} \\
Y_{H} \\
S_{L} \\
Y_{L},
\end{array}\right]
$$


where,

$$
\begin{aligned}
& X_{H}=\frac{t o l}{f l_{c}} \\
& Y_{H}=t o l \\
& S_{H}=f l_{c} \\
& X_{L}=0 \\
& Y_{L}=0 \\
& S_{L}=1 .
\end{aligned}
$$

The $a, b, c, d$ set that results from solving equation B.20 defines the cubic spline shown as a dotted line in figure B.3. This is the basic process for all four points. In fact the matrix equation, equation B.20, is the same for the other three points and now one only needs to define the set, $X_{H}, Y_{H}, S_{H}, X_{L}, Y_{L}, S_{L}$.

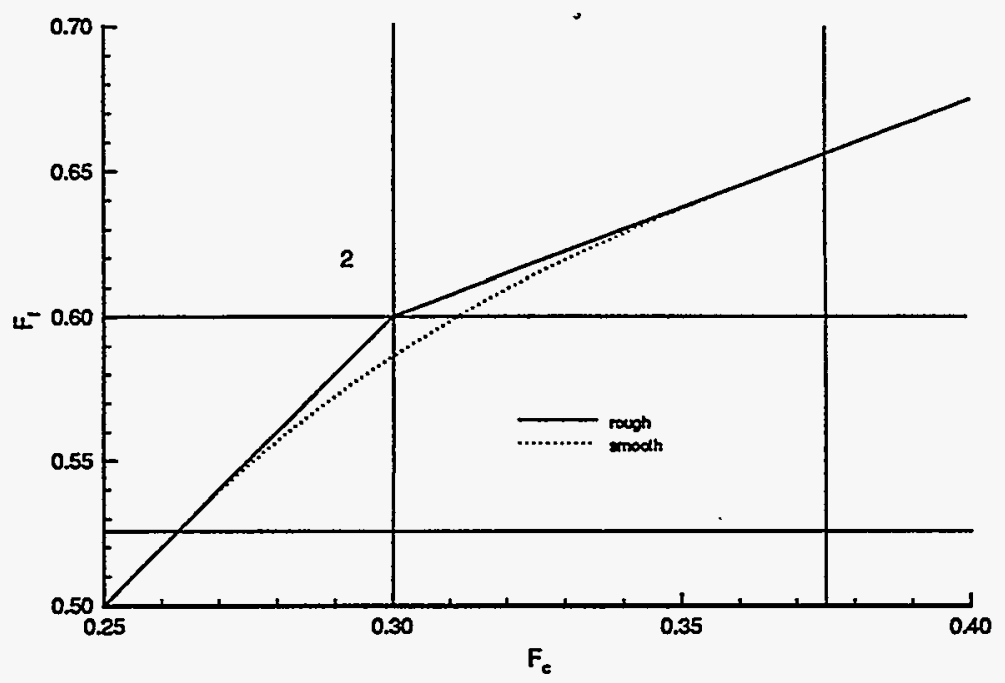

Figure B.4: Differentiable and Non-Differentiable Limiters point 2 
Figure B.4 shows the blow up of the area around point 2. The first step is to find the intersection of the two lines.

$$
\begin{aligned}
& F_{f}=f l_{c} F_{C} \\
& F_{f}=\frac{3}{4} F_{C}+\frac{3}{8} .
\end{aligned}
$$

Setting these two equations equal to each and solving results in,

$$
\begin{aligned}
f l_{c} F_{C} & =\frac{3}{4} F_{C}+\frac{3}{8} \\
F_{C 2} & =\frac{3}{8\left(f l_{c}-\frac{3}{4}\right)}=0.3 \\
F_{f 2} & =\frac{3 f l_{c}}{8\left(f l_{c}-\frac{3}{4}\right)}=0.6 .
\end{aligned}
$$

The numerical values come from $f l_{c}=2$. The slope of the line $F_{C}<0.3$ is $S_{L}=f l_{c}$, therfore this intersection will be at $Y_{L}=F_{f 2}-t o l$. Therefore $X_{L}=\frac{Y_{L}}{f l_{c}}$. The slope of the line $F_{C}>0.3$ is $S_{H}=\frac{3}{4}$, therfore $X_{H}=F_{C 2}+t o l$ and $Y_{H}=\frac{3}{4}\left(F_{C 2}+t o l\right)+\frac{3}{8}$. This defines the six numbers needed to define the cubic spline shown as a dotted line in figure B.4.

Figure B.5 shows a blow up of the area around point 3 . The first step is to find the point of intersection of the two lines.

$$
\begin{aligned}
& F_{f}=\beta F_{C}+(1-\beta) \\
& F_{f}=\frac{3}{4} F_{C}+\frac{3}{8} .
\end{aligned}
$$




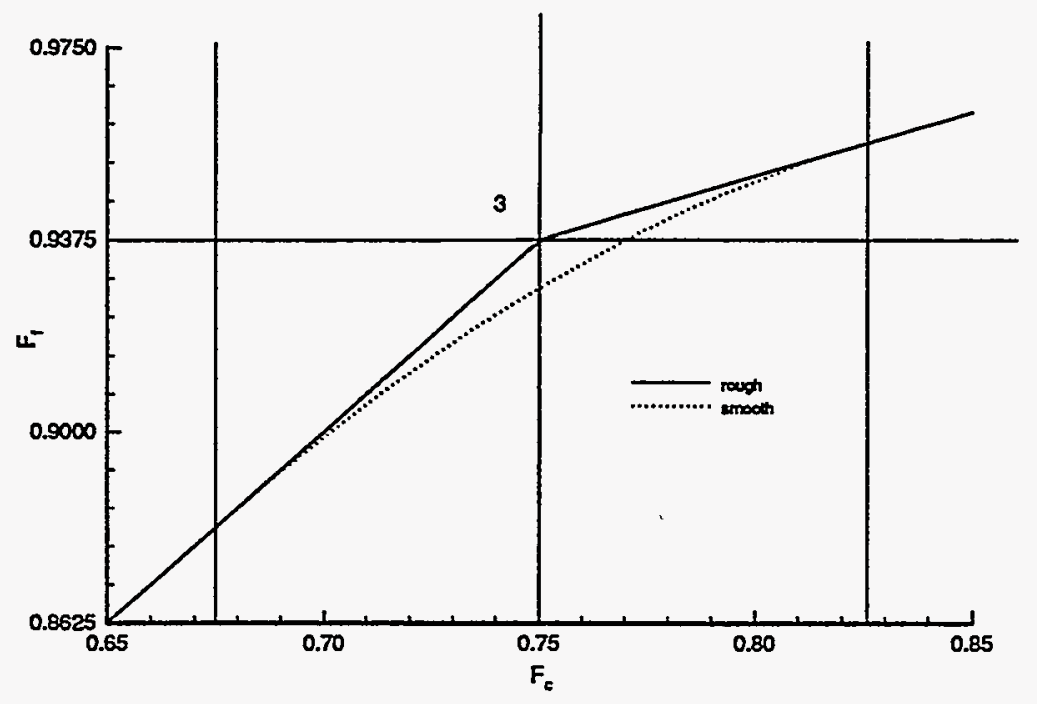

Figure B.5: Differentiable and Non-Differentiable Limiters point 3

Equating these two equations and solving for $F_{f}$ and $F_{C}$ yields,

$$
\begin{aligned}
\beta F_{C}+(1-\beta) & =\frac{3}{4} F_{C}+\frac{3}{8} \\
F_{C 3} & =\frac{5-8 \beta}{6-8 \beta}=0.75 \\
F_{f 3} & =\frac{6-9 \beta}{6-8 \beta}=0.9375 .
\end{aligned}
$$

Here the numerical values comes from using $\beta=0.25$. For the line $F_{C}<0.75$ the slope is $S_{L}=\frac{3}{4}$. Since this slope is less than $1, X_{L}=F_{C 3}-$ tol and therefore $Y_{L}=\frac{3}{4} X_{L}+\frac{3}{8}$. For the line $F_{C}>0.75$ the slope is $S_{H}=\beta$. We know that if $\beta=1$ then the limiter results in upwind differencing, therefore it is safe to assume that $\beta<1$. This implies that $X_{H}=F_{C 3}+$ tol and therfore $Y_{H}=\beta X_{H}+(1-\beta)$. This defines the two points and the two slopes used to compute the dotted line shown in figure B.5.

Figure B. 6 shows a blow up of the area around point 4. Because $S_{L}=\beta<1, X_{L}=1-t$ tol. 


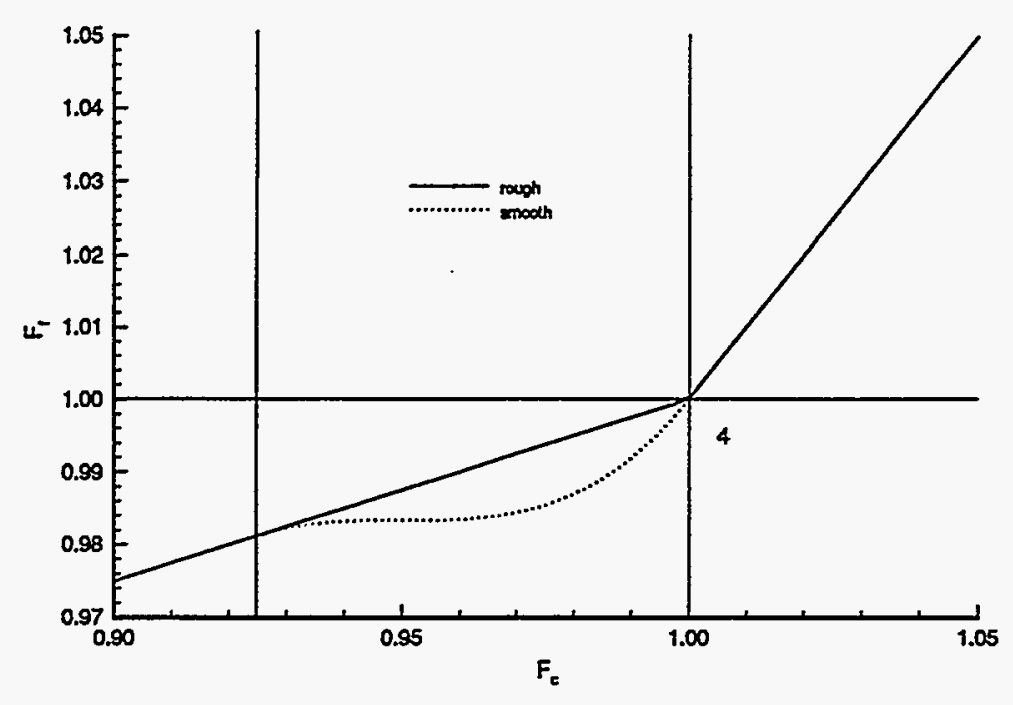

Figure B.6: Differentiable and Non-Differentiable Limiters point 4

Therefore, $Y_{L}=\beta(1-t o l)+(1-\beta)=1-\beta$ tol. From the assumptions $X_{H}=Y_{H}=S_{H}=1$.

This is all of the data necessary to compute the cubic spline shown as a dotted line in figure B.6.

\begin{tabular}{|c|c|c|c|c|c|c|c|c|}
\hline \hline Point & $X_{L}$ & $Y_{L}$ & $S_{L}$ & $X_{H}$ & $Y_{H}$ & $S_{H}$ & $X_{C}$ & $Y_{C}$ \\
\hline \hline 1 & 0 & 0 & 1 & $\frac{t o l}{f l_{c}}$ & tol & $f l_{c}$ & 0 & 0 \\
\hline 2 & $\frac{Y_{L}}{f l_{c}}$ & $Y_{C}-$ tol & $f l_{c}$ & $X_{C}+$ tol & $\frac{3}{4} X_{H}+\frac{3}{8}$ & $\frac{3}{4}$ & $\frac{3}{8\left(f l_{c}-\frac{3}{4}\right)}$ & $\frac{3 f l_{c}}{8\left(f l_{c}-\frac{3}{4}\right)}$ \\
\hline 3 & $X_{C}-$ tol & $\frac{3}{4} X_{L}+\frac{3}{8}$ & $\frac{3}{4}$ & $X_{C}+$ tol & $\beta X_{H}+(1-\beta)$ & $\beta$ & $\frac{5-8 \beta}{6-8 \beta}$ & $\frac{6-9 \beta}{6-8 \beta}$ \\
\hline 4 & $1-$ tol & $1-\beta$ tol & $\beta$ & 1 & 1 & 1 & 1 & 1 \\
\hline \hline
\end{tabular}

Table B.1: Cubic Spline Data Non-Dimensional

In conclusion, all of the data needed to create the four splines is given in Table B.1. With this information, and a method to solve equation B.20, the non-dimensional smooth limiter can be constructed. Note that the non-dimensional limiter can be calculated and saved after the input numbers $\beta$, tol, and $f l_{c}$ are know. It should be noted, that these non-dimensional 
values only need to be computed once at the beginning of the simulation.

\section{B.5 Dimensional}

In Section B.4 all variables were non-dimensionalized so there was no need to distinguish them from the dimensional variables. In this section, the tilde notation will be used (i.e., a variable with a tilde above it will be non-dimensional and all others will be dimensional). Unlike the work in the previous section, the dimensional work needs to be done for each cell every time the governing equation is evaluated.

The first step is to compute the dimensional equivalent of $X_{L}$ and $X_{H}$ for each of the four points so one can determine if the value is on the straight line or the cubic spline portion of the limiter. First recall the non-dimensionalizing equation,

$$
\tilde{F}=\frac{F-F_{U}}{F_{D}-F_{U}}
$$

For example consider $X_{L 1}=0$. This implies that,

$$
\tilde{F}_{C}=\frac{F_{C}-F_{U}}{F_{D}-F_{U}}=0
$$

Or solving for $F_{C}, F_{C}=F_{U}$. This is the equivalent dimensional point to the non-dimensional point $X_{L} 1$. Through a similar process, all of the other seven non-dimensional points can be dimensionalized. These results are shown in Table B.2.

There are two steps left now to completely define the limiter. The first step is to convert the non-dimensional cubic splines into dimensional cubic splines and the last step is to set up the dimensional IF TESTS to determine when to use the splines. The first part is very 


\begin{tabular}{|c|c|c|c|c|}
\hline \hline Point & $\tilde{F}_{C L}$ & $F_{C L}$ & $\tilde{F}_{C H}$ & $F_{C H}$ \\
\hline \hline 1 & 0 & $F_{U}$ & $\frac{t o l}{f l_{c}}$ & $F_{U}+\frac{t o l}{f l_{c}}\left(F_{D}-F_{U}\right)$ \\
\hline 2 & $\frac{3}{8\left(f l_{c}-\frac{3}{2}\right)}-\frac{t o l}{f l_{c}}$ & $F_{U}+\tilde{F}_{C L}\left(F_{D}-F_{U}\right)$ & $\frac{3}{8\left(f l_{c}-\frac{3}{2}\right)}+t o l$ & $F_{U}+\tilde{F}_{C H}\left(F_{D}-F_{U}\right)$ \\
\hline 3 & $\frac{5-8 \beta}{6-8 \beta}-t o l$ & $F_{U}+\tilde{F}_{C L}\left(F_{D}-F_{U}\right)$ & $\frac{5-8 \beta}{6-8 \beta}+t o l$ & $F_{U}+\tilde{F}_{C H}\left(F_{D}-F_{U}\right)$ \\
\hline 4 & $1-t o l$ & $F_{U}+(1-t o l)\left(F_{D}-F_{U}\right)$ & 1 & $F_{D}$ \\
\hline \hline
\end{tabular}

Table B.2: Cubic Spline Data Dimensional

straightforward. Referring back to equation B.19 and switching to the tilde notation,

$$
\tilde{F}_{f}=a \tilde{F}_{C}^{3}+b \tilde{F}_{C}^{2}+c \tilde{F}_{C}+d
$$

Dimensionalizing,

$$
\frac{F_{f}-F_{U}}{F_{D}-F_{U}}=a\left(\frac{F_{C}-F_{U}}{F_{D}-F_{U}}\right)^{3}+b\left(\frac{F_{C}-F_{U}}{F_{D}-F_{U}}\right)^{2}+c\left(\frac{F_{C}-F_{U}}{F_{D}-F_{U}}\right)+d
$$

Solving for $F_{f}$ yields,

$$
F_{f}=F_{U}+a \frac{\left(F_{C}-F_{U}\right)^{3}}{\left(F_{D}-F_{U}\right)^{2}}+b \frac{\left(F_{C}-F_{U}\right)^{2}}{\left(F_{D}-F_{U}\right)}+c\left(F_{C}-F_{U}\right)+d\left(F_{D}-F_{U}\right)
$$

One now has the dimensional form of equation $B .19$ where the $a, b, c, d$ is the same as in the non-dimensional case.

The IF TESTS have a slight complication associated with them that will be demonstrated on a sample IF TEST. From figure B.1 it is clear the if $\tilde{F}_{C}<0$ the there will be no need for a spline. Dimensionalizing, $\tilde{F}_{C}<0$ implies $\frac{F_{C}-F_{U}}{F_{D}-F_{U}}<0$. But this statement is true

$$
I F\left\{\left[\left(F_{C}-F_{U}<0\right) \text { AND }\left(F_{D}-F_{U}>0\right)\right]\right.
$$




$$
\left.O R\left[\left(F_{C}-F_{U}>0\right) \quad A N D\left(F_{D}-F_{U}<0\right)\right]\right\}
$$

Thus each non-dimensional IF TEST becomes two dimensional IF TESTS. The whole logic test looks like the following:

CHECK IF $\tilde{F}_{C}$ IS GREATER THAN ZERO

$$
\begin{aligned}
& I F\left\{\left[\begin{array}{lll}
\left(F_{D}>F_{U}\right) & A N D & \left(F_{C}>F_{U}\right)
\end{array}\right]\right. \\
& \left.O R\left[\left(F_{D}<F_{U}\right) A N D\left(F_{C}<F_{U}\right)\right]\right\} T H E N
\end{aligned}
$$

CHECK IF $\tilde{F}_{C}$ IS LESS THAN ONE

$$
\begin{aligned}
& I F\left\{\left[\left(F_{D}>F_{U}\right) \quad A N D \quad\left(F_{C}<F_{D}\right)\right]\right. \\
& \left.O R\left[\left(F_{D}<F_{U}\right) A N D\left(F_{C}>F_{D}\right)\right]\right\} T H E N
\end{aligned}
$$

CHECK IF $\tilde{F}_{C}$ IS CLOSE TO POINT 4

$$
\begin{array}{ccc}
I F\left\{\left[\left(F_{D}>F_{U}\right)\right.\right. & A N D & \left.\left(F_{C}>F_{C L 4}\right)\right] \\
\text { OR }\left[\left(F_{D}<F_{U}\right)\right. & A N D & \left.\left.\left(F_{C}<F_{C L 4}\right)\right]\right\} T H E N \\
& \text { USE SPLINE 4 }
\end{array}
$$

$E N D I F$

CHECK IF $\tilde{F}_{C}$ IS CLOSE TO POINT 1

$$
I F\left\{\left[\left(F_{D}>F_{U}\right) \quad A N D \quad\left(F_{C}<F_{C H 1}\right)\right]\right.
$$




$$
\begin{gathered}
O R\left[\left(F_{D}<F_{U}\right)\right. \\
\text { USE SPLINE } 1
\end{gathered}
$$

$E N D I F$

CHECK IF $\tilde{F}_{C}$ IS CLOSE TO POINT 3

$$
\begin{array}{ccl}
I F\left\{\left[\left(F_{D}>F_{U}\right)\right.\right. & A N D & \left(F_{C}>F_{C L 3}\right) \\
& A N D & \left.\left(F_{C}<F_{C H 3}\right)\right] \\
O R\left[\left(F_{D}<F_{U}\right)\right. & A N D & \left(F_{C}<F_{C L 3}\right) \\
\cdot \quad A N D & \left.\left.\left(F_{C}>F_{C H 3}\right)\right]\right\} T H E N \\
\text { USE SPLINE 3 } &
\end{array}
$$

ENDIF

CHECK IF $\tilde{F}_{C}$ IS CLOSE TO POINT 2

$$
\begin{array}{lll}
I F\left\{\left[\left(F_{D}>F_{U}\right)\right.\right. & A N D & \left(F_{C}>F_{C L 2}\right) \\
& A N D & \left.\left(F_{C}<F_{C H 2}\right)\right] \\
O R\left[\left(F_{D}<F_{U}\right)\right. & A N D & \left(F_{C}<F_{C L 2}\right) \\
& \text { AND } & \left.\left.\left(F_{C}>F_{C H 2}\right)\right]\right\} T H E N \\
\text { USE SPLINE 2 } &
\end{array}
$$

$E N D I F$

ENDIF

LESS THAN ONE

$E N D I F$

GREATERTHAN ZERO 
In conclusion, at input time the non-dimensional numbers can all be calculated. Then each time a face value is needed the normal "rough" limiter is used, and subsequently the previous set of IF TESTS are used to see if the face value needs smoothing. 


\section{Bibliography}

[1] J. M. Dawson. Computer modeling of plasma: Past, present and future. Physics of Plasmas, Vol. 2, No. 6:2189-2199, June 1995.

[2] S.I. Braginskii. Transport Processes in a Plasma. In M.A. Leontovich, editor, Reviews of Plasma Physics Volume 1, pages 205-311. Consultants Bureau, New York, 1965.

[3] C. K. Birdsall and A. B. Langdon. Plasma Physics via Computer Simulation. McGrawHill, 1985.

[4] M. N. Rosenbluth, W. M. MacDonald, and D. L. Judd. Fokker-Plank equation for an inverse-square force. Phys. Rev., Vol. 107:1-6, 1957.

[5] J. Killeen, G. D. Kerbel, M. G. McCoy, and A. A. Mirin. Computational Methods for Kinetic Models of Magnetically Confined Plasmas. Springer-Verlag, 1986.

[6] A. Lenard and I. B. Bernstein. Plasma oscillations with diffusion in velocity space. Physical Review, Vol. 112 No. 5:1456-1459, 1958.

[7] P. J. Catto. Personal communication, Dec. 1993.

[8] P. J. Catto. Personal communication, Oct. 1994.

[9] S. I. Braginskii. Transport phenomena in a completely ionized two-temperature plasma. Soviet Physics Journal of Experimental and Theoretical Physics, Volume 6 (33) Number 2:358 - 369, February, 1958.

[10] L. Landau. Kinetic equation in the case of Coulomb interaction. Soviet Physics Journal of Experimental and Theoretical Physics, 7:203-209, 1937.

[11] D.L. Book. NRL Plasma Formulary. Naval Research Laboratory, Washington, DC 20375-5000, 1990. NRL Publication 177-4405.

[12] F.F. Chen. Introduction to Plasma Physics and Controlled Fusion Volume 1: Plasma Physics. Plenum, 1990.

[13] A. R. Bell. Non-Spitzer heat flow in a steadily ablating laser-produced plasma. Phys. Fluids, Vol. 28 No. 6:2007-2014, 1985.

[14] P. J. Drallos and M. E. Riley. Boltzmann simulations of RF discharges. unpublished.

[15] D. B. Graves. Plasma processing. IEEE Transactions on Plasma Science, Vol. 22, NO. 1:31-42, Feb. 1994. 
[16] M. F. Leahy. Superfine IC geometries. IEEE Spectrum, pages 36-43, Feb. 1985.

[17] L. M. Montierth, R. L. Morse, and W. A. Neuman. Collisional transport treatment of surface plasma structures. Phys. Fluids B, Vol. 1, No. 9:1911-1925, Sept. 1989.

[18] R. J. Procassini and D. A. Knoll. Kinetically motivated boundary conditions for fluid models of scrape-off layer transport. Journal of Nuclear Materials, 196-198:363-368, 1992.

[19] D. Loffhagen and R. Winkler. A new nonstationary Boltzmann solver in self-consistent modelling of discharge pumped plasmas for excimer lasers. Journal of Computational Physics, Vol. 112:91-101, 1994.

[20] L. Spitzer. Physics of Fully Ionized Gasses. John Wiley and Sons, Inc., 1962.

[21] L. Spitzer and R. Harm. Transport phenomena in a completely ionized gas. Physical Review, Volume 89, Number 5:977-981, March 1, 1953.

[22] F. S. Felber. Steady-state model of a flat laser-driven target. Physical Review Letters, Volume 39, Number 2:84-87, July 11, 1977.

[23] C. E. Max, C. F. McKee, and W. C. Mead. A model for laser driven ablative implosions. Physics of Fluids, Vol 23, No 8:1621-1645, August 1980.

[24] R. J. Procassini, C. K. Birdsall, and B. I. Cohen. Particle simulations of collisional transport in a high recycling, diverted tokamak scrape-off layer. Nuclear Fusion, Vol. 30, No. 11:2329-2348, 1990.

[25] R. J. Mason. Hybrid and collisional implicit plasma simulation models. In J. U. Brackbill and B. I. Cohen, editors, Multiple Time Scales, chapter 8, pages 233-270. Academic Press, 1985.

[26] O. V. Batischev, S. I. Krasheninnikov, D. J. Sigmar, Yu. S. Sigov, and T. K. Soboleva. Influence of kinetic effects on particle and energy flows in the ITER divertor. In K. H. Spatschek et al., editors, Contributions to Plasma Physics Vol. 34 Number 2/3, pages 436-441. Akademie Verlag, 1994.

[27] D. P. Coster. Multi-species, two-velocity, one-space Fokker-Planck code. In A. K. Prinja, editor, U.S. Edge Plasma Physics: Theory and Applications Workshop, 1993.

[28] J. D. McCullen, L. M. Montierth, R. L. Morse, and W. A. Neuman. Surface plasma structures in the kinetic regime. Phys. Fluids B, Vol. 1, No. 2:448-467, Feb. 1989.

[29] R. J. Procassini and C. K. Birdsall. Particle simulation model of transport in a bounded, Coulomb collisional plasma. Phys. Fluids B, Vol. 3, No. 8:1876-1891, Aug. 1991.

[30] Z. Abou-Assaleh, J.P. Matte, T.W. Johnston, and R. Marchand. Fokker-Planck modelling of edge plasma near the neutralizer plate in a tokamak. Contrib. Plasma Phys., 32 3/4:268-272, 1992.

[31] D. A. Knoll, A. K. Prinja, and R. B. Campbell. A direct Newton solver for the twodimensional tokamak edge plasma fluid equations. Journal of Computational Physics, 104:418-426, 1993. 
[32] D.A. Knoll, P.R. McHugh, and V.A. Mousseau. Newton-Krylov-Schwarz methods applied to the tokamak edge plasma fluid equations. In D.E. Keyes, Y. Saad, and D.G. Truhlar, editors, Domain-Based Parallelism and Problem Decomposition Methods in Computational Science and Engineering (Minnesota Supercomputer Institute, April 2526,1994, pages 75-95. SIAM, 1995.

[33] D.A. Knoll and P.R. McHugh. An Inexact Newton Algorithm for Solving the Tokamak Edge Plasma Fluid Equations on a multiply connected domain. J. Comp. Phys., 116:281291, 1995.

[34] L. A. Hageman and D. M. Young. Appled Iterative Methods. Academic Press, 1981.

[35] E. M. Epperlen. Implicit and conservative difference scheme for the Fokker-Planck equation. Journal of Computational Physics, 112:291-297, 1994.

[36] S. I. Krasheninnikov, T. K. Soboleva, O. V. Batischev, V.D. Levchenko, P.A. Ovchenkov, Yu. S. Sigov, I.I. Silaev, and G.I. Zmievskaya. Kinetic modeling of the transport processes in the tokamak edge plasma. In Contributions to Plasma Physics Vol. 32 Number 3/4, pages 273-242, 1992.

[37] O. Sauter, R. W. Harvey, and F. L. Hinton. A 3-d Fokker-Planck code for studying parallel transport in tokamak geometry with arbitrary collisionalities and application to neoclassical resistivity. In K. H. Spatschek et al., editors, Contributions to Plasma Physics Vol. 34 Number 2/3, pages 169-174. Akademie Verlag, 1994.

[38] D. P. Coster. Tokamak Divertor Modeling with Fluid and Kinetic Codes. PhD thesis, Princeton University, 1993.

[39] L. R. T. Gardner, G. A. Gardner, and S. I. Zaki. Collisional effects in plasmas modelled by a simplified Fokker-Planck equation. Journal of Computational Physics, Vol. 107:40$50,1993$.

[40] C. E. Rathmann and J. Denavit. Simulation of collisional effects in plasmas. Journal of Computational Physics, Vol. 18:165-187, 1975.

[41] J. J. Duderstadt and L. J. Hamilton. Nuclear Reactor Analysis. Wiley, 1976.

[42] E. L. Vold, A. K. Prinja, F. Najmabadi, and R. W. Conn. The neutral diffusion approximation in a consistent tokamak edge plasma-neutral computation. Journal of Nuclear Materials, 176 \& 177:570-577, 1990.

[43] G. A. Emmert, R. M. Wieland, A. T. Mense, and J. N. Davidson. Electric sheath and presheath in a collisionless, finite ion temperature plasma. Phys. Fluids, Vol. 23 No. 4:803-812, 1980.

[44] P. J. Roache. Computational Fluid Dynamics. Hermosa Publishers, 1985.

[45] G. Dahlquist and A. Bjorck. Numerical Methods. Prentice-Hall, Inc., 1974.

[46] B. C. Char et al. Maple V Language Reference Manual. Springer-Verlag, 1991. 
[47] B. M. Averick, J. J. More, C. H. Bischof, A. Carle, and A. Griewank. Computing large sparce Jacobian matrices using automatic differentiation. SIAM J. Sci. Comput., Vol. 15 No. 2:285-294, 1994.

[48] R. Dembo, S. C. Eisenstat, and T. Steihaug. Inexact Newton methods. SIAM Journal Numerical Analysis, 19:400-408, 1982.

[49] W.L. Briggs. A Multigrid Tutorial. Society for Industrial and Applied Mathematics, 1987.

[50] A. Brandt. Multigrid techniques: 1984 guide with applications to fluid dynamics. Technical report, von Karman Institute, 1984.

[51] R.W. Freund, G.H. Golub, and N.M. Nachtigal. Iterative solution of linear systems. Acta Numerica, pages 57-100, 1991.

[52] P. R. McHugh. An Investigation of Newton-Krylov Algorithms for Solving Incompressible and Low Mach Number Compressible Fluid Flow and Heat Transfer Problems Using Finite Volume Discretization. $\mathrm{PhD}$ thesis, University of Idaho, 1995.

[53] P. R. McHugh and D. A. Knoll. Inexact Newton's method solutions to the incompressible Navier-Stokes and energy equations using standard and matrix-free implementations. In Proceedings of the 11th AIAA Computational Fluid Dynamics Conference: Part 1, page 385, Orlando,FL, July 1993.

[54] P. R. McHugh and D. A. Knoll. Comparison of standard and matrix-free implementations of several newton-krylov solvers. AIAA, Vol. 32, No. 12:2394-2400, December 1994.

[55] J. A. Meijerink and H. A. van der Vorst. Guidelines for the usage of incomplete decompositions in solving sets of linear equations as they occur in practical problems. $J$. Comput. Phys., Vol. 44:134-155, 1981.

[56] P. N. Brown and A.C. Hindmarsh. Matrix-free methods for stiff systems of ODE's. SIAM Journal of Numerical Analysis, 23:610-638, 1986.

[57] P. N. Brown and Y. Saad. Hybrid Krylov methods for nonlinear systems of equations. SIAM Journal of Scientific and Statistical Computing, 11:450-481, 1990.

[58] J. W. Watts. A conjugate gradient-truncated direct method for the iterative solution of the reservoir simulation pressure equation. Society of Petroleum Engineers Journal, pages 345-353, June 1981.

[59] Y. Saad and M.H. Schultz. GMRES: A generalized minimal residual algorithm for solving non-symmetric linear systems. SIAM Journal Scientific Statistical Computing, $7: 856,1986$.

[60] B.P. Leonard. Eliptic systems: Finite-difference method IV. In W.J. Minkowycz, E.M. Sparrow, G.E. Schneider, and R.H. Pletcher, editors, Handbook of Numerical Heat Transfer, chapter 9, pages 347-378. Wiley, 1988.

[61] J.S. Chang and G. Cooper. A practical difference scheme for Fokker-Planck equations. Journal of Computational Physics, 6:1-16, 1970. 